\title{
RELATIONSHIP BETWEEN TYPES OF SOCIAL SUPPORT, COPING STRATEGIES, AND \\ PSYCHOLOGICAL DISTRESS IN INDIVIDUALS LIVING WITH CONGENITAL HEART DISEASE
}

by

MI-YEON KIM

Bachelor of Nursing, University of Manitoba, 1984

Master of Science in Nursing, McGill University, 1998

\author{
A THESIS SUBMITTED IN PARTIAL FULFILLMENT OF \\ THE REQUIRMENTS FOR THE DEGREE OF \\ DOCTOR OF PHILOSOPHY \\ in \\ The Faculty of Graduate Studies \\ (Nursing)
}

THE UNIVERSITY OF BRITISH COLUMBIA

(Vancouver)

December 2012

(C) Mi-Yeon Kim, 2012 


\begin{abstract}
Many survivors of congenital heart disease (CHD) are confronted with various medical complications and psychosocial issues arising from limitations related to the disease. They often feel that they are "being controlled by the disease" and experience feelings of depression and anxiety. The purpose of this study was to examine the relationship between types of social support, coping strategies, and psychological distress in individuals living with CHD. The study tested a model that explained psychological distress in the study population. A cross-sectional survey design was used to recruit 272 participants from the Adult CHD program clinic at a tertiary care hospital in Western Canada. The Mplus statistical software was used to analyze the data. Structural equation modeling was used to estimate a parsimonious model and goodness-of-fit indices were used to test the fit of the model with the data. The estimation and testing of two models were performed separately for the two outcomes of psychological distress, namely depression and anxiety. The results were similar for both anxiety and depression. One of the key findings was the impact of social support on psychological distress. Perceived social support was directly related to both anxiety and depression. Received social support influenced anxiety and depression but its effect was through perceived social support. Wishful-thinking coping strategies mediated the relationship between perceived social support and both anxiety and depression. The only difference evident between anxiety and depression was the partial mediation effect of problem-solving coping strategies on the relationship between perceived social support and depression; in anxiety, the same partial mediation effect of problem-solving coping strategies was absent. Findings of the study suggest that individuals with low perceived and received
\end{abstract}


social support are especially vulnerable to experiences of psychological distress.

Furthermore, individuals with low perceived social support tend to use more wishfulthinking coping strategies, which in turn, tend to increase their level of anxiety and depression. The findings imply that assessments of social support and type of coping strategies used are an integral part of the nursing care of adults living with CHD. 


\section{Preface}

This study required ethics approval. The approval for this study was provided by the University of British Columbia-Providence Health Care Research Ethics Board (UBCPHC REB). The Ethics Certificate number of the UBC-PHC REB is H10-01388. 


\section{Table of Contents}

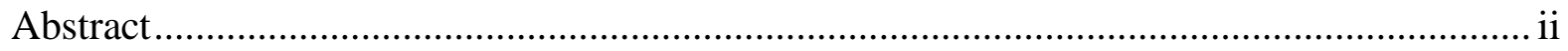

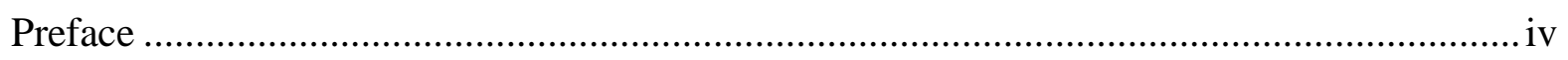

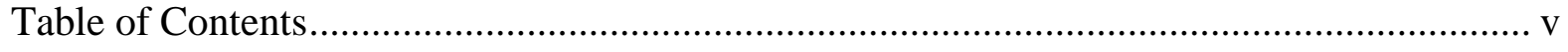

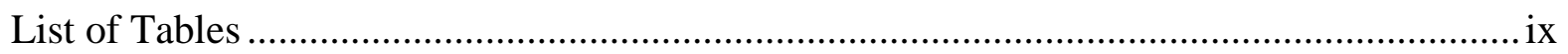

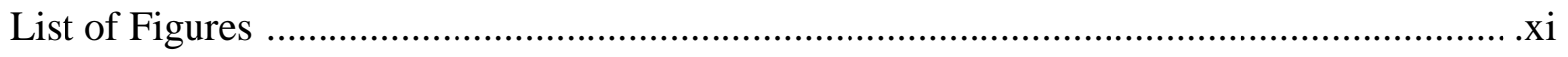

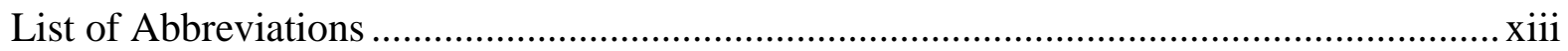

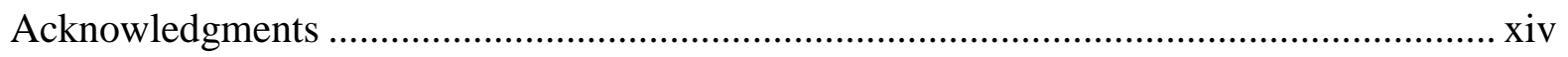

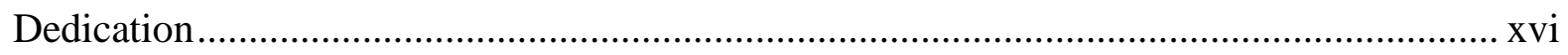

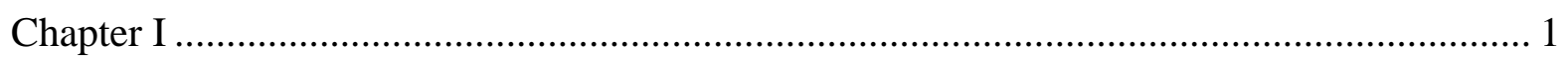

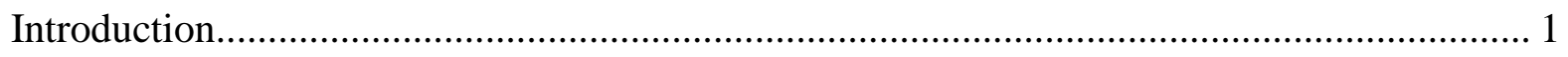

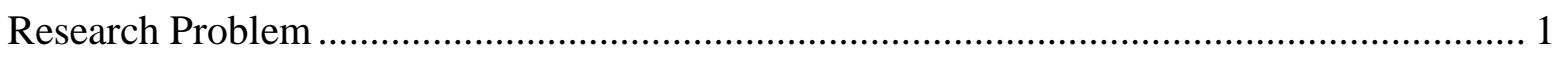

Adult Congenital Heart Disease ..................................................................... 3

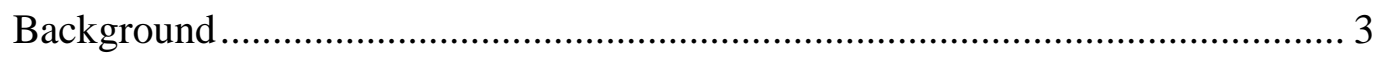

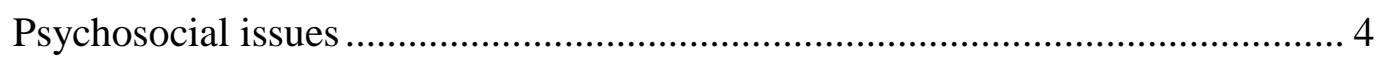

Contributing factors of adaptation ........................................................ 7

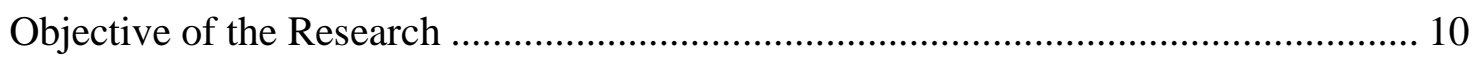

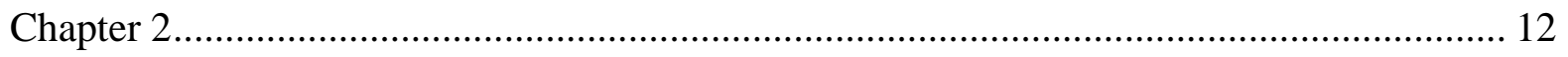

Review of the Literature and Proposed Conceptual Framework ...................................... 12

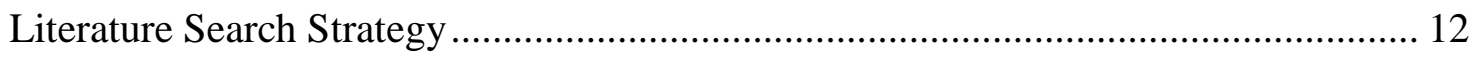

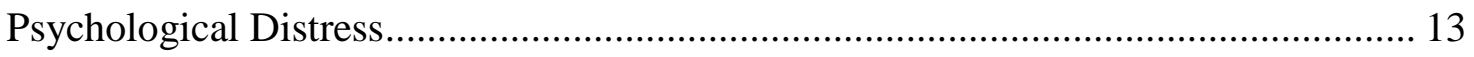

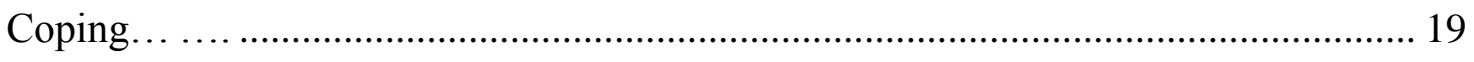




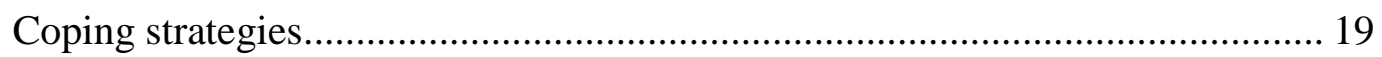

Relationship between coping strategies and psychological distress .................... 24

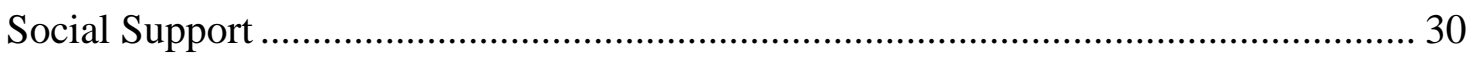

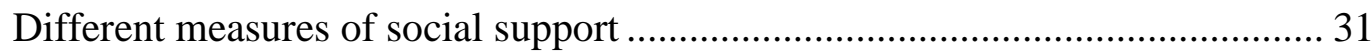

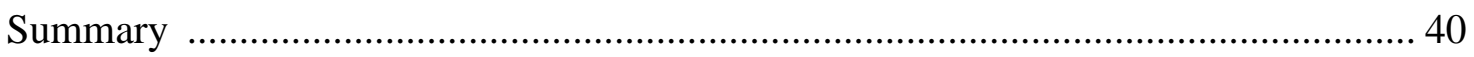

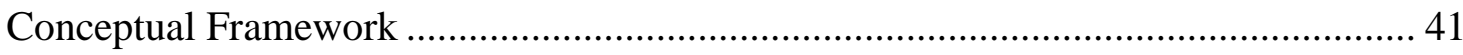

Psychological distress (anxiety and depression) ................................................. 41

Relationships between psychological distress and social support ....................... 43

Relationship between coping strategies and psychological distress.................... 44

Relationships between social support and coping ............................................ 46

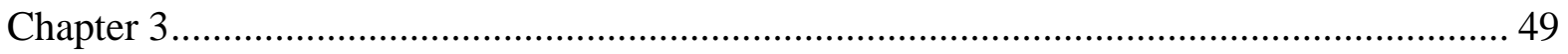

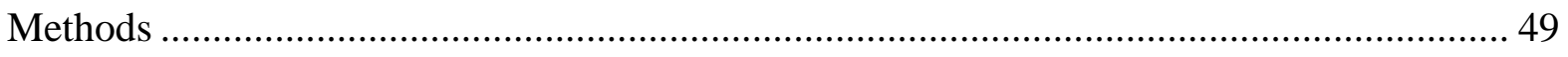

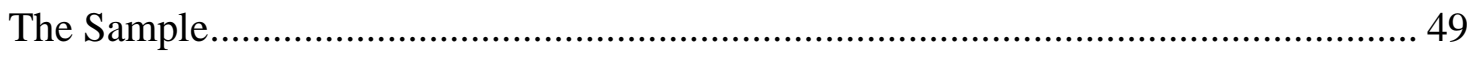

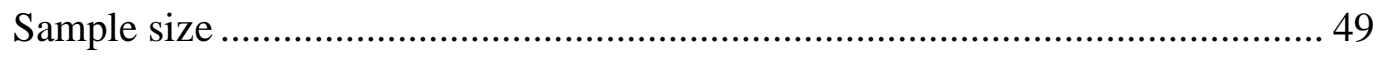

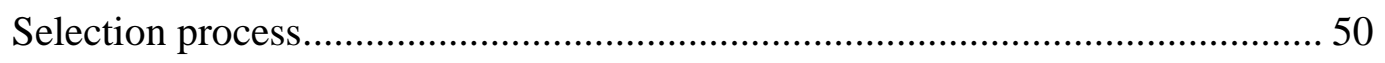

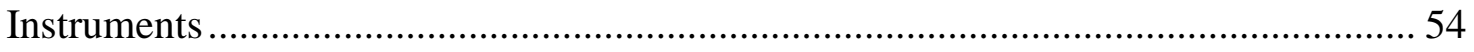

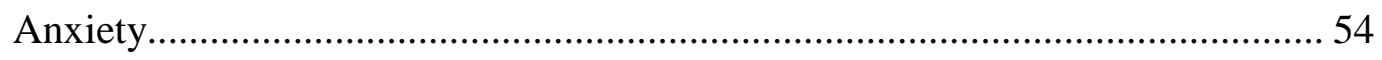

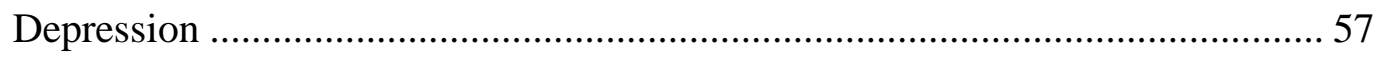

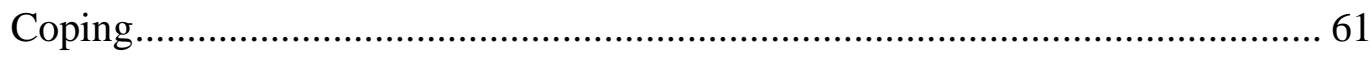

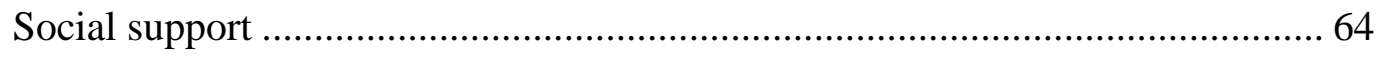

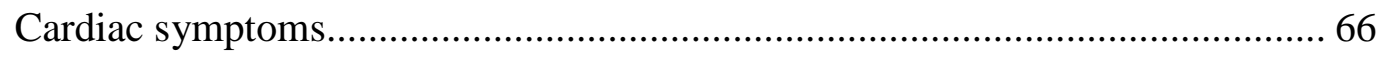

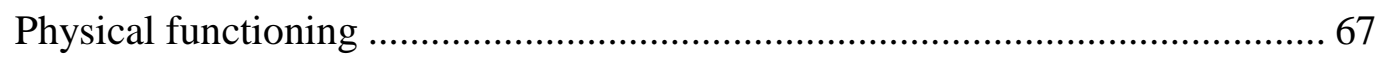

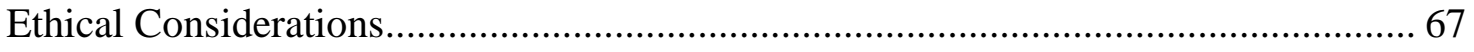




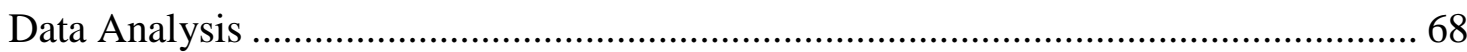

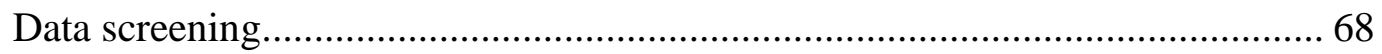

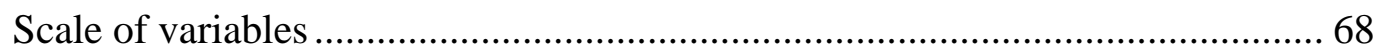

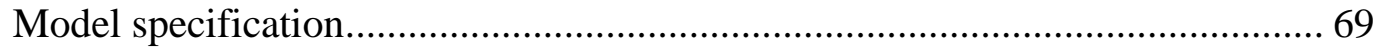

Step one: Testing of measurement models ……………………………..... 70

Step two: Testing of the structural models ................................................... 71

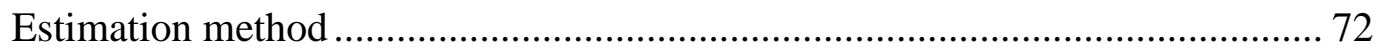

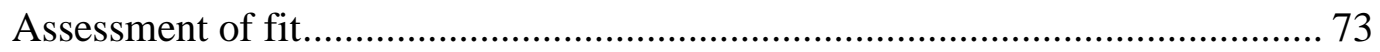

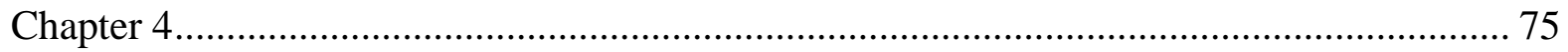

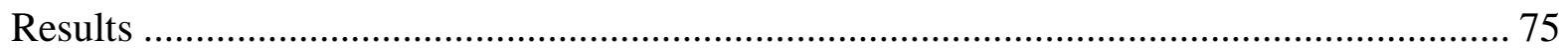

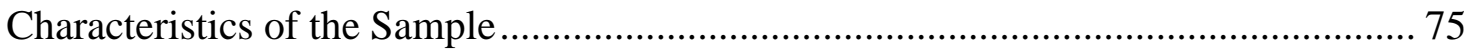

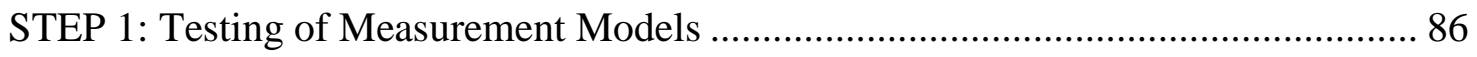

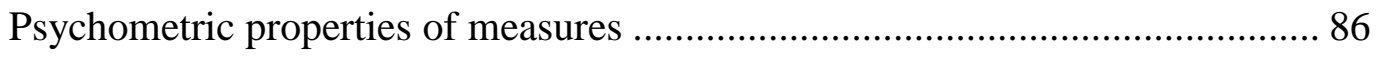

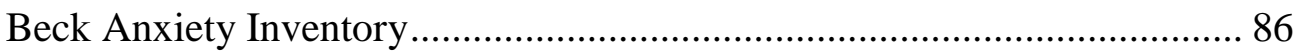

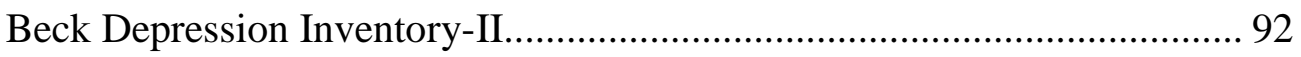

Ways of Coping Questionnaire................................................................. 92

Berlin Social Support Scales (BSSS) ………………………………..... 111

Examination of missing data ..................................................................... 120

STEP 2: Testing of the Structural Models ............................................................... 121

Findings of structural equation modeling ……………....................................121

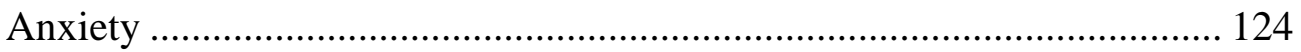

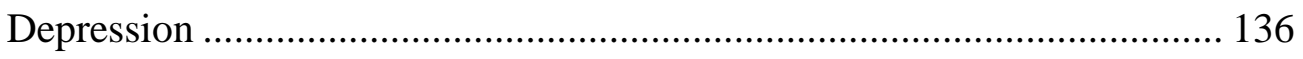

Chapter 5 


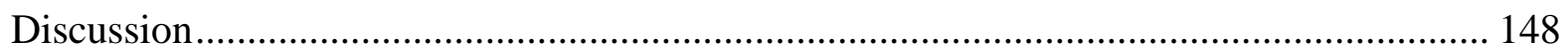

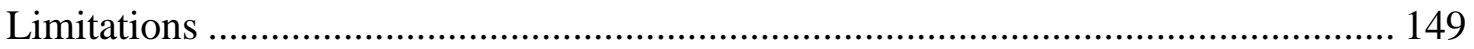

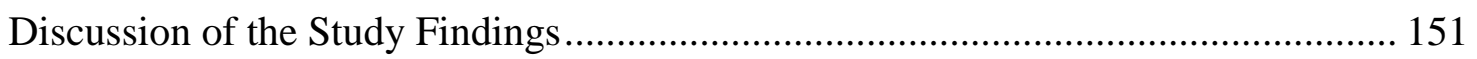

Relationship between types of social support and psychological distress......... 151

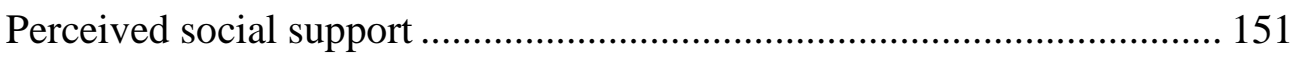

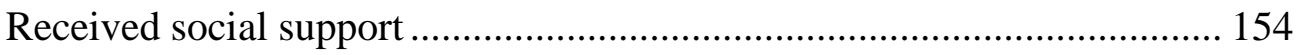

Relationship between types of social support and psychological distress

mediated by different coping strategies............................................................. 161

Perceived social support and coping strategies ....................................... 1611

Coping strategies and psychological distress ........................................... 163

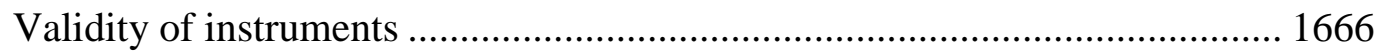

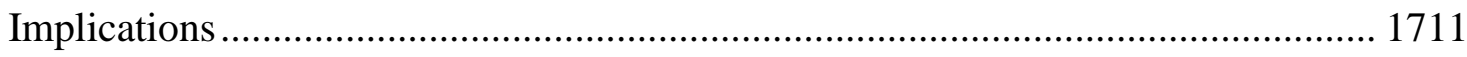

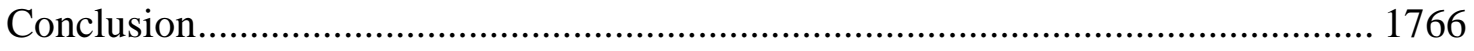

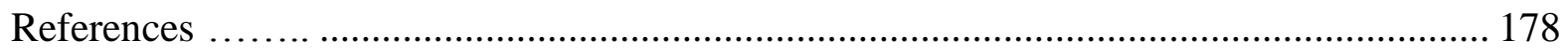

Appendix A (Letter of Invitation \& Consent Form) ......................................................... 203

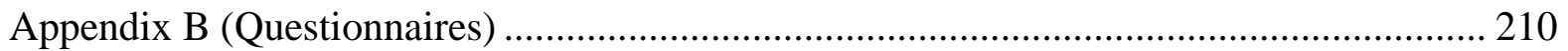




\section{List of Tables}

Table 1 Sociodemographic Characteristics of the Participants................................. 76

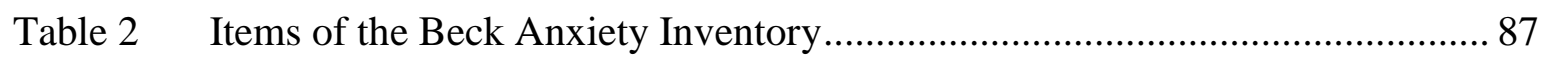

Table 3 Factor Models of BAI Described in the Literature ...................................... 89

Table 4 Process of Selecting the Subjective Subscale of BAI Model Proposed by Osman et al. (1997) ................................................................ 90

Table 5 Items of the Beck Depression Inventory-II............................................ 93

Table 6 Factor Models of BDI-II Described in the Literature ................................. 94

Table 7 Factor Models of Ways of Coping Instrument Described in the

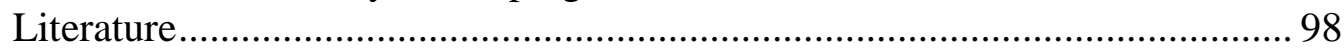

Table 8 The Factor Structure of the Three Subscales of Ways of Coping Questionnaire Established With the Data From the Current Study ............... 107

Table 9 Items of the Perceived Social Support Subscale...................................... 112

Table 10 Berlin Social Support Scale: Perceived Social Support............................... 114

Table 11 Items of the Received Social Support Subscale ...................................... 117

Table 12 Berlin Social Support Scale: Received Social Support ............................ 118

Table 13 Frequency of Missing Data in Study Variables ....................................... 121

Table 14 Estimated Correlation Matrix for the Latent Variables .............................. 123

Table 15 Model 1: The Effects of Types of Social Support and Types of Coping Strategies on Anxiety.......................................................................... 126

Table 16 Model 2: The Effects of Perceived Social Support and Types of Coping Strategies on Anxiety With the Constraint of Received Social Support.

Table 17 The Chi-Square Test of the Difference in Fit Between the Model 1 (Effects of Received Social Support are Freely Estimated) and the Model 2 (Effects of Received Social Support are Constrained).... 130 
Table 18 Model 3: The Effects of Received Social Support on Anxiety

Mediated by Perceived Social Support........................................................... 134

Table 19 Model 4: The Effects of Types of Social Support and Types of

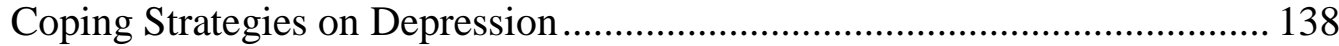

Table 20 Model 5: The Effects of Perceived Social Support and Types of Coping Strategies on Depression With the Effects of Received

Social Support Constrained

Table 21 The Chi-Square Test of the Difference in Fit between the Model 4 (Effects of Received Social Support are Freely Estimated) and the Model 5 (Effects of Received Social Support are Constrained)

Table 22 Model 6: The Effect of Received Social Support on Depression

Mediated by Perceived Social Support.............................................................. 145 


\section{List of Figures}

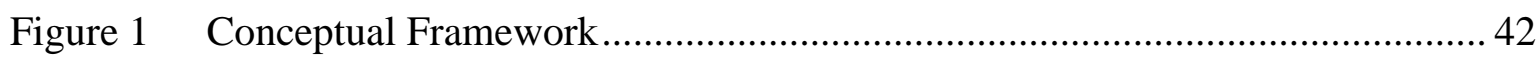

Figure 2 Selection Process Through Direct Contact at the ACHD Clinic .................... 52

Figure 3 Selection Process Through Mail-out Questionnaire ..................................... 53

Figure 4 Types and Frequency Of Common Cardiac Symptoms Experienced by The Sample (Measured With the Cardiac Symptom List)............................. 80

Figure 5 Short Form (SF-36)v2 Health Survey (Physical Functioning Subscale)......... 82

Figure 6 Proposed Measurement Model for BAI-Subjective Subscale to be Used for SEM in This Study ................................................................... 91

Figure 7 Proposed Measurement Model for Cognitive-Affective Subscale of the BDI-II to be Used for SEM in This Study.......................................... 95

Figure 8 The Scree Plot of Eigenvalues Extracted from the Ways of Coping Questionnaire 106

Figure 9 Proposed Measurement Model of Three Subscales of WCQ Problem-Solving, Wishful-Thinking, Denial/Avoidance - to be Used in This Study With the ACHD Data....

Figure 10 Proposed Measurement Model of Perceived Social Support to be Used in This Study With the ACHD Data

Figure 11 Proposed Measurement model of Received Social Support to be Used in This Study With the ACHD Data.

Figure 12 Model 1: The Effects of Social Support (Perceived \& Received) and Coping Strategies (Problem-Solving, Wishful-Thinking and Denial/Avoidance) on Anxiety.

Figure 13 Significant Paths for Model 1: The Effects of Social Support (Perceived \& Received) Coping Strategies (Problem-Solving, Wishful-Thinking and Denial/Avoidance) on Anxiety 
Figure 14 Significant Paths for Model 2: The Effects of Social Support (Perceived \& Received) Coping Strategies (Problem-Solving, Wishful-Thinking and Denial/Avoidance) on Anxiety With the Effects of Received Social

Support Constrained

Figure 15 Model 3: The Effect of Received Social Support on Anxiety

Mediated by Perceived Social Support

Figure 16 Significant Paths for Model 3: The Indirect Effects of Perceived Social Support on the Relationship between Received Social Support and Anxiety.

Figure 17 Model 4: The Effects of Social Support (Perceived \& Received) Coping Strategies (Problem- Solving, Wishful-Thinking and Denial/Avoidance) on Depression

Figure 18 Significant Paths for Model 4: The Effects of Social Support (Perceived \& Received) and Coping Strategies (Problem Solving, Wishful Thinking and Denial/Avoidance) on Depression 139

Figure 19 Significant Paths for Model 5: The Effects of Social Support (Perceived \& Received) and Coping Strategies (Problem-Solving, Wishful-Thinking and Denial/Avoidance) on Depression With the Effects of Received Social Support Constrained 143

Figure 20 Model 6: The Effects of Received Social Support on Depression Mediated by Perceived Social Support

Figure 21 Significant Paths for Model 6: The Indirect Effects of Perceived Social Support on the Relationship between Received Social Support and Depression.... 


\section{List of Abbreviations}

ACHD

ADL

AIDS

BAI

BDI

BSSS

BC

CA-S

CFA

CFI

CHD

CI

CSL

df

DWLS

EFA

EPC

G-S-C

HIV

HRSD

MI

MI

ML

MLM

NNFI

OR

RMSEA

SA-C

SD

SEM

SF-36v2

TLI

ULS

W

WCCL

WCQ

WLSMV

WLS
Adult congenital heart disease

Activities of daily living

Acquired immune deficiency syndrome

Beck Anxiety Inventory

Beck Depression inventory

Berlin Social Support Scales

British Columbia

Cognitive-affective and somatic

Confirmatory factor analysis

Comparative fit index

Congenital heart disease

Confidence interval

Cardiac Symptom List

Degrees of freedom

Diagonally weighted least squares

Exploratory factor analysis

Expected parameter change

General-somatic-cognitive

Human immunodeficiency virus

Hamilton Rating Scale for Depression

Modification indices

Myocardial infarction

Maximum likelihood

Robust maximum likelihood

Non-normed fit index

Odds ratio

Root mean square error of approximation

Somatic-affective and cognitive

Standard deviation

Structural equation modeling

Short-Form Health Survey-36 version 2

Tucker-Lewis fit Index

Unweighted least squares

Matrix

Ways of Coping Checklist

Ways of Coping Questionnaire

Mean- and variance-adjusted weighted least square

Weighted lease squares 


\section{Acknowledgements}

It was not until I engaged in the research of my dissertation that I fully began to grasp the gratitude that researchers express to their study participants. I was truly taken by surprise at the willingness of many participants to extend their time to "help others who may be in need". Had it not been for their generous sharing of their time, the results of the study would not have been fruitful. Many participants included letters or wrote memos describing their life stories of living with congenital heart disease and many expressed their lives as being not different from those who are unaffected with congenital heart condition. In the midst of long hours spent collecting data, the feelings of gratitude and appreciation I felt toward study participants kept me anchored and motivated.

My sincere and heart felt appreciation is extended to my supervisor Dr. Joy Johnson whom I feel privileged to have been guided by. I have appreciated her expert knowledge of the research process and the support she has provided me as a mentor. With gentle encouragement always reflected in a positive light, she taught me the importance of striving for quality research and the independence of a scholarly researcher. I would also like to express my sincere gratitude to Dr. Carol Jillings for not only her critical review and feedback of the study, but also for her guidance and support in the initial phase of my doctoral studies. Dr. Richard Sawatzky was invaluable for his assistance with the statistical analysis of the data and for the insightful comments and feedback on the results of the study. Had it not been for his knowledge of the statistical program, I would still be stuck in a sea of complex statistical analyses. I would also like to thank Stephanie, my editor, without whom my grammatical presentation of this thesis would have not been at the caliber of a $\mathrm{PhD}$ student. She offered consistent support and always came through during the times of anxiety-stricken tight deadlines.

I would like to express my appreciation for Dr. Marla Kiess of the Adult Congenital Heart Disease (ACHD) Program at St. Paul's Hospital who allowed me access to her patients at the ACHD clinic. I would like to express my gratitude for the assistance the clinical nurse specialist, Ms. D. Fofonoff, of the ACHD program provided. I would also like to thank the staff nurses and support staff of the ACHD program who generously assisted with the collection of my data.

I am deeply thankful for my family -especially my mom and opa (오빠)- who have encouraged and supported me with love and patience throughout the long journey of my doctoral studies.

Most importantly, I give praise and thanks to the LORD (Yahweh RŌ'i). 
I wish to acknowledge that this study was supported by the FUTURE Program for Cardiovascular Nurse Scientists, UBC School of Nursing Research Grants and Langara College Research Fund. 


\title{
Dedication
}

\author{
To my sister, \\ Bo-Yeon Kim
}

Thank you for taking my side even when I was at fault.... 


\section{Chapter I}

\section{Introduction}

\section{Research Problem}

The inception of this study began when I started to wonder about the factors that influence the psychosocial outcomes of individuals living with chronic health challenges. More specifically, I pondered the specific elements that differentiated "well-adjusted" individuals from "poorly-adjusted" individuals with chronic disease. Evidence increasingly points to the inadequacy of the biomedical model to account for the complex multifaceted processes involved in human adaptation. Research findings have also identified the need to look beyond the treatment of physiological aspects of physical disability, to examine the psychosocial dimensions associated with psychosocial adjustment.

"She knows that she needs the anticoagulant for her life but she just won't take it. I just don't know why!" (verbal communication, Dr. M. Kiess, 2005)

The sense of frustration expressed by Dr. Kiess, a cardiologist, reflects that the behaviors of people are influenced by factors others than those required for sustaining life. A comprehensive understanding of individuals affected with medical conditions and disabilities requires the examination of various psychosocial factors that influence adjustment.

Psychological distress (including depression and anxiety) has been found to have a significant relationship with the health of people with physical illness. The prevalence of depression in people affected with medical illness varies between 15-61\% (Martucci, Balestrieri, \& Bisoffi, 1999). Depression is associated with increased rates of morbidity and 
mortality. A meta-analysis by Cuijper and Smits (2002) found that the relative risk of mortality is 1.81 (95\% CI 1.58-2.07) in depressed individuals in comparison to nondepressed individuals. Furthermore, depression is related to both the onset and the accelerated progression of diseases such as coronary heart disease, diabetes, cancer, chronic pain, end-stage renal disease, and obesity (Steptoe, 2007). For example, the findings from the Baltimore Epidemiologic Catchment Area study indicated that the probability of stroke is twice as high in depressed individuals than in non-depressed individuals. Depression is also a contributing factor to the evolution of atherosclerosis (carotid plaque) (Jones, Bromberger, Sutton-Tyrrell, \& Matthews, 2003). Studies have shown that depressed individuals exhibit less willingness and capacity to adhere to a treatment regimen than non-depressed individuals (Bosley, Fosbury \& Cochrane, 1995).

Emotional distress other than depression has also been linked to the incidence of coronary heart disease, particularly in men. Studies have shown that independent of cardiac risk factors and demographic variables, psychological distress is associated with the development of future coronary heart disease (i.e., death, MI) (Mendes de Lone, Krumholz, \& Seeman, 1998).

When health challenges impact the day-to-day lives of individuals living with a chronic illness such as congenital heart disease (CHD), clearly psychosocial as well as physical factors need to be examined in order to understand how individuals manage issues related to their chronic disease. The primary aim of this research, therefore, was to examine some of the important predictors of psychological distress, particularly depression and anxiety, experienced by individuals with congenital heart disease. 


\section{Adult Congenital Heart Disease}

\section{Background.}

Congenital heart disease (CHD) is a structural abnormality of the heart or great vessels that can affect functional aspects of people's lives. The prevalence of congenital heart disease is estimated to be 4-12 per 1,000 live births, or approximately $1 \%$ of all live births (Hoffman \& Kaplan, 2002; Lip, Lane, \& Millane, 2008). Until 1938, surgical intervention for children with CHD was non-existent, and few children survived beyond adolescence (Cohen, 1992). Today, 350,000 Canadians (Statistics Canada, 2006) and almost 1 million Americans live with congenital heart disease, and with advancing medical and surgical management of the disease, survivors are maturing to adulthood at a rate of $5 \%$ per year (Kovacs, Sears, \& Saidi, 2005).

As the lifespan of people living with congenital heart disease grows, researchers are beginning to examine psychosocial adjustment and various life issues faced by this unique group of people. Despite the increased life expectancy, many survivors are confronted with various medical complications including arrhythmias, heart failure, haematological action, and pulmonary hypertension (Engelfriet et al., 2005; Warnes et al., 2001). As a result, many of them require life-long follow up and medical attention. According to a report by the U. S. Agency for Healthcare Research and Quality (2007), the cost of health care associated with hospitalization of individuals with CHD was $\$ 1.4$ billion in 2004. In addition, many survivors face various psychosocial issues resulting from living with this serious disease. For example, some survivors, particularly those with a complex heart defect, face limited choices in employment related to their physical disability (Green, 2004). Even when survivors of CHD do get hired, it is often into positions with which they are not satisfied as 
they may be unable to perform certain job-related tasks due to the limitations imposed by the heart defect. Because of their heart defect, many survivors also face difficulty in obtaining medical (in USA) and/or life insurances (Hart \& Garson Jr., 1993; Niwa et al., 2002). Often, for survivors of CHD in the USA, the only way to obtain medical insurance is to be employed by a company that offers group health coverage. Female survivors of CHD face additional unique challenges related to reproduction. Some women with cyanotic heart diseases are advised against bearing children, and as a result, face the dilemma of having to choose between bearing biological children and preserving their health (Lyon, Kuehl \& McCarter, 2006). Additionally, many people living with CHD experience limitations in terms of their physical capacity and the amount of physical activities they are able to perform. Due to these restrictions and more, many survivors of CHD report feeling "being controlled by the disease" in most aspects of their lives (Claessens, Moons, Dierckx, de Casterle, Budts, \& Gevilling, 2005).

\section{Psychosocial issues.}

Despite successful surgical repair experienced during childhood, research has shown that many individuals with CHD are less adaptable to change and may experience feelings of persistent insecurity, depression, anxiety, and low self-esteem (Livecchi, 2004; Rosenthal, 1993). Furthermore, although some studies report no differences between a group of individuals with CHD and their reference peers (Moons et al., 2006; Utens et al., 1994; Utens et al., 1998), there remain a group of survivors who experience a varied level of psychological distress related to living with CHD (Bromberg, Beasley, D’Angelo, Landzberg, \& DeMaso, 2003; Cohen, Mansoor, Langut, \& Lorber, 2007; Spurkland, Bjornstad, 
Lindberg, \& Seem, 1993). Kovacs, Silversides, and Saidi (2005) reported that 35-79\% of participants in their study experienced psychological distress. Similarly, in a sample of individuals labelled "well-adjusted" by their medical team, 35\% exhibited emotional distress (Bromberg et al., 2003). Children and adolescents with CHD frequently experience psychological distress including anxiety disorder, depression, dysthymia, and fear of the unknown (Bjornstad, Spurkland, \& Lindberg, 1995; Gupta, Giuffre, \& Waters, 1998). The prevalence of anxiety attacks experienced by people with CHD is $10 \%$, which is lower than the 2-6\% rate of the general population. In extreme instances, an ideation of suicide among persons with CHD has also been noted (Popelova, Slavik, \& Skovranek, 2001).

Adults with CHD report worse emotional outcomes of adjustment in comparison to their peers without heart defects (Kovacs, Sears, \& Saidi, 2005). They report experiencing difficulty in family and peer interactions as a result of their heart defects (e.g., parental overprotection) (Kovacs, Silversides, Saidi, \& Sears, 2006). Horner et al. (2000) observed a persistent denial among their study subjects with CHD during the interviews. Subjects only revealed, when questioned extensively, their true feelings about the difficult social interactions they experienced during childhood and adolescence. Many of these interviewees admitted to coping with negative experiences by "putting on a happy face" or displaying an attitude of "everything is fine". Adolescents and young adults, particularly males, are sensitive to the appearance of their surgical scars or other physical signs of heart disease (e.g., cyanosis, scoliosis, clubbing of fingers) (Gantt, 1992; Horner, Kovacs, Sears, \& Saidi, 2005; Kovacs, Sears, \& Saidi, 2005; Horner, Liberthson \& Jellnek, 2000; Thomason, 1997; Uzark, Mones, Slusher, Limbers, Burwinkle, \& Varni, 2008). While females are able to cover their sternotomy scars with clothing and cyanotic nails with nail polish, fewer covering options are 
available to men, particularly in hot weather when people tend to wear less clothing. This factor offers a possible explanation as to the heightened distress reported by young males. Further, young adults, particularly those considering sexual intimacy, are ambivalent about disclosing their heart defect (Claessens et al., 2005; Uzark, 1992). As a result, many individuals who are worried about rejection from sexual partners chose to conceal their condition.

Several themes related to the psychosocial experience of individuals with CHD have been identified in research: normality, social integration, body image, disclosure, uncertainty, dependence, and coping. The central theme of the lives of these individuals, particularly young adults, is the notion of "feeling different" (Claessens et al., 2005; Horner et al., 2000; Wright et al., 1985). Factors such as physical limitation, medication intake, and frequent visits to the medical team continually remind survivors that their lives are different from those without CHD (McMurray et al., 2001). Because of their perceived sense of being different, many of them also feel "left out" especially when they cannot physically keep up in activities with their peers or partners (Claessens et al., 2005; Jefferies, Noonan, Keller, Wilson, \& Griffith III, 2004). Further, physical limitations have led to survivors of CHD being teased, humiliated, and/or subjected to painful nicknames (Kovacs et al., 2005).

In some instances people with CHD are not told of the potential future medical complications or the possibility of repeat surgery. Thus, they grow up with the assumption that they are "cured" only to find out that they require lifelong medical follow-ups (Warnes, 2005). The progressive nature of their heart defect exposes patients to an uncertain future and they are often left with feeling "betrayed" and distressed related to the presumed negative outcome (Verstappen, Pearson, Kovacs, 2006). Some survivors continue to live at 
home with their parents due to either the severity of their disease (especially neurological complication) and/or parental overprotection (Garson, Williams, \& Reckless, 1974;

Kokkonen \& Paavilainen, 1992). To compensate for feelings of abandonment, social isolation, and inferiority, some survivors keep their feelings private (Horner et al., 2000), or choose to push their physical boundaries by engaging in destructive behavior (e.g., drinking, smoking, body tattoos) (Cetta, Graham, Lichtenberg, \& Warnes, 1999). Survivors of CHD employ a variety of coping strategies to deal with living with this chronic illness. This issue will be addressed in more detail in a later section.

\section{Contributing factors of adaptation.}

As an increasing number of individuals with CHD are surviving longer as a result of advancements in technology and medical management, researchers are beginning to examine factors that contribute to adaptation in this unique group of people. Intuitively, an assumption can be made that patients with complex congenital heart lesions would be particularly vulnerable to poor psychosocial adaption in comparison to those with mild congenital heart lesions. However, the existing evidence is conflicting and does not always support this conjecture (Brandhagen, Feldt, \& Williams, 1991). Research has identified a number of factors that were found to influence human adaptation in the face of a stressful event. More specifically, variables such as social support and coping strategies have been linked to psychological distress in individuals with chronic health challenges.

Psychological distress is defined as "the general concept of maladaptive psychological functioning in the face of stressful life events" (as cited in Ridner, 2004, 
p. 539). More specifically, psychological distress refers to "the unpleasant subjective states of depression and anxiety, which have both emotional and physiological manifestations" (Mirowsky \& Ross, 2003, p. 8). The emotional component is called 'mood, and the physiological component is called 'malaise'. Anxiety is characterized by feelings of worry or fear, and malaise of headaches, dizziness, or stomach aches, whereas depression is characterized by feelings of sadness or worthlessness, and malaise of listlessness and distraction. Many researchers use measures of anxiety and/or depression as the primary indicator of mental health (i.e., psychological distress) (Barnett, 1993). In the past, researchers measured psychological distress using indicators that focused on malaise (i.e., cold sweats, heart palpitation), partly due to the concern that participants may feel disturbed or reluctant about reporting their emotional states (Mirowsky \& Ross, 2003), presumably because of the societal stigma associated with mental illness. However, the discovery was made that people were neither troubled nor made uneasy by being directly asked about their emotions. Moreover, people are increasingly becoming interested in the relationship between physical and psychological health as studies reveal that physiological factors alone are insufficient to account for variation in health. As a result, the current research focused questions more on mood or emotions (i.e., depressed, fearful, worried, or sad) than physiological symptoms of psychological distress.

Psychological distress as an outcome in individuals living with CHD is an important variable that needs to be studied because of its relationship with health outcomes (i.e., increased mortality, cardiac disease, and the noncompliance with the medical regimen) (Mazari et al., 2005; Rosengren, Hawken, \& Ounpuu, 2004). 
Social support is one of the variables that has received extensive attention in research as a possible contributing factor to the observed variance in health outcomes. Evidence suggests that social support yields a protective or stress-buffering function in individuals' adaptation. In other words, the provision of support that leads people to perceive that they are cared for, loved, valued, and worthy of care is linked to positive health outcomes (Lindsey \& Yates, 2004). Initially, social support was conceptualized as a unidimensional construct. However, researchers have since described the construct as a multidimensional concept that is commonly categorized based on its structure (source, duration), functions (emotional, instrumental, appraisal, informational), disposition (perceived vs. received support), and nature (positive vs. negative evaluation); different aspects of social support are associated with different health outcomes. For example, Wells, Booth-Jones and Jacobsen (2009) have found that instrumental support is negatively related to depression in recipients of hematopoietic stem cell transplantation. In other words, patients who received a greater level of instrumental support experienced a decreased level of depression, whereas patients who received a decreased level of support experienced an increased level of depression. Some researchers have suggested the importance of differentiating the disposition of support - perceived vs. received - because of a potential incongruence between the perception of support and the actual support provided and their differential effects on the health outcome (Stewart, 1989; Wills \& Shinar, 2000).

Coping is another variable that has been extensively studied in relation to stressful events and adaptational outcomes (depression, psychological symptoms). Coping refers to cognitive and behavioral efforts to manage the stressor that are appraised as exceeding the resources of an individual (Lazarus, 1984). Folkman and Lazarus (1985) describe two 
modes of coping: problem-focused coping (modify or change the stressor causing the problem) and emotion-focused coping (regulate the psychological distress caused by the stressor). Cognitive appraisal, the cardinal concept in stress and coping theory (Folkman \& Lazarus, 1984), consists of primary appraisal (evaluation of what is at stake) and secondary appraisal (evaluation of one's resources that can used to prevent harm or improve benefit). Folkman and Lazarus suggest that coping should have a fit with the given situation, in other words, the type of coping that one uses should be appropriate to the situation of the stressor. Thus, problem-focused coping would be more suited for situations that are amenable to change, whereas emotion-focused coping would be more suited for situations in which nothing can be done (Folkman \& Lazarus, 1988). Indeed, Forsythe and Compas (1987) have reported that an adaptive outcome was observed in individuals who used more problemfocused than emotion-focused coping in situations that were appraised as controllable. In contrast, individuals who used more emotion-focused coping than problem-focused coping in situations that were appraised as uncontrollable demonstrated an adaptive outcome.

\section{Objective of the Research}

The number of adults with CHD is increasing quickly. Much research has been conducted on the medical management of CHD. And, advancing technology related to both surgical and medical management is rapidly decreasing the mortality and morbidity of the disease. Further, workforce description and practice guidelines have been developed for the medical care of adults with CHD (Child et al., 2001). However, these practice descriptions and guidelines fail to address many of psychosocial issues confronting the population and clearly, studies indicate that these individuals are experiencing varying levels of depression 
and psychological distress. Little is known about how these individuals manage or adjust in the psychosocial dimensions of their lives. Knowledge is urgently required to generate pertinent and practical information that can serve as a basis for developing and providing interventions that would address the various needs of, and to assist with the optimization of psychosocial adjustment of people living with CHD.

The purpose of this study was to examine the relationships among different types of social support (i.e., perceived and received social support), coping strategies (i.e., problemand emotion-focused), and psychological distress (depression and anxiety) in individuals living with CHD. In particular, the focus was to build and test a parsimonious model that best explained the relationships between social support, use of coping strategies, and psychological distress. 


\section{Chapter 2}

\section{Review of the Literature and Proposed Conceptual Framework}

In this chapter, a review of the literature relevant to each of the study variables is

presented. The examination of the existing evidence begins with the outcome variable of psychological distress, followed by social support and coping strategies. The discussion of the research findings serves to identify the gaps in our understanding of psychological adjustment of people living with $\mathrm{CHD}$ and informs the conceptual framework that guides this study. Prior to the discussion of literature, the strategies used to identify research findings pertaining to the study variables are presented.

\section{Literature search strategy}

Electronic databases including Medline (1970-present), CINAHL (1981-present), PsychINFO (1982-present), and EMBASE (1980-2008) were searched for published literature. Non-peer reviewed information was primarily limited to dissertation manuscripts. The strict limitation was not applied to the criteria of published year as the early descriptive studies of CHD provide important insight into the initial psychological outcomes in this group of people. Bibliographies of selected studies and books were hand searched to identify additional studies that may provide relevant information. The search terms used were: congenital heart disease, adult congenital heart disease (ACHD), depression, anxiety, emotional distress, psychological distress, coping, coping strategy, coping style, self-efficacy, social support, psychosocial adjustment, cardiac disease, chronic disease, and chronic disease management. Initially, the search was limited to the context of CHD and Adult Congenital 
Heart Disease (ACHD) to examine the prevalence of psychosocial issues and psychological distress in population with CHD. Based on the identified issues in CHD literature (e.g., social support, coping), each variable was then searched separately in the context of both CHD and the other chronic health conditions (e.g., search terms combined as CHD and chronic diseases). Because the literature dealing with psychosocial predictor variables specific to the population of CHD was limited, the search had to be expanded to include the environment of other various chronic diseases: rheumatoid arthritis, diabetes, chronic obstructive lung disease, burns, and cystic fibrosis. People with these physical disorders were thought to share common challenges associated with living with chronic illnesses, such as CHD. In the following section, each of the study variables is examined in the context of research findings.

\section{Psychological Distress}

In the literature, the use of the term psychological distress is commonly interchanged with emotional distress. Regardless of which term is chosen to express the distress state of an individual, the measures of sadness, anxiety, hopelessness, worthlessness, listlessness, and distractions reflect interchangeable indications of distress. For the purpose of this study, the term 'emotional distress' is viewed as synonymous with 'psychological distress'. In addition, the discussion of psychological distress as an outcome variable is presented with a primary focus on depression and anxiety.

The phenomenon of depression in people with CHD is better understood in the context of different depression theories. Theories of depression provide a variety of perspectives from which to analyze and understand the phenomenon of depression. Beck's 
cognitive theory describes depression as arising from dysfunctional attitudes in which themes of loss, inadequacy, failure, and worthlessness predominate. Dysfunctional attitudes involve the notion that one's happiness and worth depend on being perfect or on gaining other people's approval (Abramson et al., 2002; Jarrett, 1990). Abramson's hopelessness theory, which is similar to Beck's theory, postulates that depressive symptoms arise as a result of feeling hopeless. A person who is feeling hopeless is at risk for vulnerability when he or she attributes the cause of a stressor to the following factors: internal (cause is within self), global (likely affects many areas of life), and stable (likely to persist over time) (Abramson et al., 2002). From the perspective of psychoanalytic theory, depression is seen as "an unusual susceptibility to dysphoric feelings; a vulnerability to feelings of loss and disappointment; intense need for contact and support; a proclivity to assume blame and responsibility; and a liability to feelings of guilt" (Blatt, D'Afflitti, \& Quinlan, 1976). In an attempt to integrate various formulations of depression, Blatt proposed two dimensions of depression: anaclitic and introjective depression (Blatt, 2004). Anaclitic depression is characterized by a dysphoric mood arising from fears of being abandoned, and by wishes to be cared for, loved, and protected. The primary feelings in anaclitic depression reflect helplessness, weakness, depletion, and being unloved. Introjective depression is characterized by feelings of worthlessness, inferiority, and guilt, with self-criticism as the central theme. The sense that one has failed to live up to expectations and standards predominates in introjective depression (Blatt, 2004, Blatt et al., 1976).

Anxiety is a complex phenomenon characterized by feelings of fear, apprehension, and worry, and is often associated with physical symptoms such as palpitations, chest pain, dizziness, and/or shortness of breath (Fan \& Shi, 2009). Anxiety refers to an aversive 
experience of distress that denotes emotional, attitudinal, cognitive, physiological, perceptual, and behavioral responses (Williams, 1995). The essence of anxiety is being in a state of feeling fearful (William, 1987). The sensation of feeling anxious involves a subjective judgment of fear intensity, ranging on a continuum from not afraid to extremely afraid. Anxiety encompasses an array of anxiety disorders including: panic disorder/attacks, generalized anxiety disorder, agoraphobia, specific phobia, social phobia, obsessivecompulsive disorder, and post-traumatic stress disorder. Research on anxiety commonly distinguishes between state and trait anxiety (Spielberger, 1972). State anxiety refers to a transitory response involving unpleasant emotional arousal and apprehensive thoughts when facing threatening demands or danger. Trait anxiety, on the other hand, denotes the presence of stable individual differences in one's tendency to respond with state anxiety in the anticipation of threatening circumstances.

A number of theories of anxiety offer explanatory frameworks from which to study the phenomenon: psychoanalytic and psychodynamic theory, behavioral theories, and cognitive theories. The psychodynamic theory explains symptoms as reflective of underlying conflicts. Behavioral theories broadly emphasize that a fear is acquired after the repeated exposure to the frightening stimulus, or by observing others' reactions to feargenerating stimuli. Social Cognitive theory proposed by Bandura (1994) explains that selfefficacy plays a pivotal role in anxiety arousal. Anxiety is not aroused when people believe they can control the perceived threat, but rather, in situations where they perceive that they lack the ability to manage the threat. Fan and Shi (2009), however, caution to avoid focusing exclusively on psychopathological theories of anxiety to study the phenomenon. They claim that the normal anxiety people deal with day-to-day is also important to understand because 
the failure to effectively cope with "normal" anxiety can disrupt life and eventually develop into an anxiety disorder. Anxiety exerts an effect on health risk behavior. For example, anxious individuals may delay seeking health care or, avoid health screenings (e.g., HIV testing, mammogram ) (Schwarzer, 1994).

In the context of the adult congenital heart disease (ACHD) population, normal dayto-day anxiety as well as destructive anxiety disorders are prevalent. Kovacs et al. (2009) reported in their study involving 280 adult patients with CHD that $26 \%$ of their participants met the criteria for an anxiety disorder (generalized anxiety disorder, panic disorder, social phobia, specific phobia, or obsessive-compulsive disorder). Similarly, Bromberg et al. (2003) found in their study that $36 \%$ of their participants $(n=22)$ were experiencing anxiety.

Studies have revealed some of the factors that are known to influence feelings of anxiety and depression experienced by people with CHD. When the life experience of individuals living with CHD is examined, many themes reported by participants in various studies echo the statements described in depression and anxiety theories. For example, many individuals report experiencing feelings of inferiority and helplessness related to their inability to "keep up" in activities with their peers; rejection related to visible signs from surgeries and signs of heart diseases (e.g., cyanosis); and worthlessness related to the difficulty in finding a satisfactory job or obtaining life insurance. Some adolescents and young adults feel guilty about the origin and the progressive deterioration of their heart disease, where they view their illness as a punishment for bad actions or thoughts (Masi \& Brovedani, 1999). It is not difficult then to identify factors inherently associated with the origin of depression and comprehend how these factors may contribute to the development of psychological distress. 
The influence of various physical illnesses on depression has been extensively documented in literature. Chronic illnesses such as cardiac disease (Adsett \& Bruhn, 1968; Burker, Evon, Losielle, Finkel, and Mill, 2005; Doehrman, 1977, Frasure-Smith \& Lesperance, 2005), cancer (Simon, Palmer \& Coyne, 2007), and HIV/AIDS (Namir, Wolcott, Fawzy, \& Alumbaugh, 1987; Perez, Chartier, Koopman, Vosvick, Felton, \& Spiegel, 2009) have been widely documented as influencers in the development of depression. The danger of psychological distress such as depression is even greater when the circular effect of the phenomenon is recognized. The relationship between depression and chronic illnesses seems to operate bidirectionally, in other words, depression can assume the contributing role for the development of chronic illness or a dire physical outcome. For example, studies have shown that depression is a significant risk factor for mortality, especially in people with cardiac disease. Frasure-Smith et al. (2000) examined the relationship between depression and mortality in 887 patients following a myocardial infarction (MI). Findings indicated that depressed individuals at baseline were at a significantly increased risk of one-year cardiac mortality ( $\mathrm{OR}=3.6 ; 95 \% \mathrm{CI}=1.68$ to $6.70 ; \mathrm{p}=0.0006)$. Similarly, Mazari et al. (2005) conducted a four-year prospective, community based cohort study to examine the relationship between depressive symptomatology and mortality in a community of 5,632 members. They found that after controlling some of the potential covariates, depressed men were twice as likely (HR, 2.02; 95\% CI, 1.58-2.8), and women were almost one and half times as likely (HR,1.43; 95\% CI, 1.04-1.95) to die at the four year follow-up in comparison to their counterparts without depressive symptoms.

Substantial evidence has demonstrated that emotionally distressed individuals are at risk for developing cardiac illness. For example, a multicentre study with 11,000 first-time 
MI patients and matched control persons recruited from 52 countries revealed that depressive symptoms were more common in MI patients than the control (Yusuf et al., 2004). In addition, depression also influences the recovery trajectory of individuals affected with chronic illnesses, e.g., non-compliance with medical management (Rosengren, Hawken, \& Ounpuu, 2004). Blumenthal (1982) examined 35 persons after a MI to assess the relationship between depression and compliance with a post-MI exercise regimen. Results demonstrated an inverse relationship between depression and exercise adherence. Similar findings were reported by Carney (1995) who studied 55 individuals with coronary disease and found that depressed participants were less likely to adhere to medication treatment. In contrast, however, Botelho and Dudrak (1992) found that depression did not influence medication adherence in 55 patients with coronary artery disease.

Given the link between psychological distress and dire health consequences, researchers have identified a number of external and internal variables that are associated with emotional outcomes in people with chronic illness, including: social support and coping. However, no studies to date have examined these variables and their impact on people with CHD. Existing studies examining psychosocial adjustment of people with CHD are primarily descriptive in nature and do not sufficiently inform health care providers on the optimal interventions that are required for this unique group of individuals. In the following section, a discussion is presented on the various factors that are predictive of psychological outcomes, in particular, emotional distress in people living with CHD. Influencing variables of social support and coping in relation to psychological distress are presented by examining various research findings pertaining to each factor. 


\section{Coping}

In this study, coping refers to the cognitive and behavioral attempts to manage stressors, and a coping strategy refers to the specific coping response the individual employs to deal with the stressors. Prior to reviewing the literature on the relationship between coping strategies and psychological distress, a discussion will be presented on the different types of coping strategies and how people use them to deal with stressors in their environment. The influence of coping on psychosocial outcomes needs to be examined in the context of the theoretical underpinnings of the coping process.

\section{Coping strategies.}

In the past three decades, much interest has focused on the processes by which the general population, particularly individuals affected by chronic illnesses, responds and adapts to various stressful encounters within the environment. The coping process of humans is complex and inherently multifaceted. Existing research has generally concentrated on two primary areas: 1) the generation of coping process classification and 2) the influence of coping processes on adjustment outcomes. Theoretical perspectives such as the stress and coping theory by Lazarus (1966) enable an in-depth examination and analysis of the adjustment process for people living with physiological challenges.

The original method of categorizing coping responses described in the stress and coping theory consists of two main categories: problem-focused coping and emotion-focused coping (Auerbach, 1989; Folkman \& Lazarus, 1984). Problem-focused coping refers to cognitive or behavioral strategies directed toward dealing with a stressor by modifying, avoiding, or minimizing the problem. Emotion-focused coping, on the other hand, refers to 
strategies directed toward attenuating or eliminating dysphoric emotions elicited by a stressor. Lazarus has further refined the categorization system by distinguishing between the method (active vs. passive) and the focus (problem-focused vs. emotion-focused) of coping (Burker, Evon, Sedway, \& Egan, 2005). Since then, others have proposed different ways of classifying coping responses in an attempt to be explicit and discriminative. For example, Billings and Moos (1981) suggested the typology consisted of 1) active-behavioral strategies (attempts to deal directly with the stressor, e.g., "tried to find out more about the situation"), 2) active-cognitive strategies (attempts to modify or change cognitive appraisal of the stressor, e.g., "tried to see the positive side of the situation"), 3) avoidance strategies (attempts to avoid confronting the stressor or to engage in behaviors to decrease tension, e.g., “drinking or smoking more”) (Holahan \& Moos, 1987). Irrespective of coping response classification systems, the clear implication is that people cope through behavior ("doing") and/or thoughts ("thinking").

The principles used to differentiate responses to a stressor become clear when each of the specific coping strategies is examined in detail. Although various authors have presented different sets of coping strategies to study people's coping processes, the COPE Inventory introduced by Carver and colleagues describes one of the most exhaustive sets of coping strategies (Carver, Scheier, \& Weintraub, 1989). The COPE Inventory includes the following thirteen strategies that Carver et al. claim are conceptually distinct: "active coping, planning, suppression of competing activities, restraint coping, seeking instrumental social support, seeking emotional social support, focusing on and venting of emotions, behavioral disengagement, mental disengagement, positive reinterpretation and growth, denial, acceptance, and turning to religion" (p. 268-270). Based on identified strategies, the COPE 
Inventory assesses both the coping appraisal (e.g., positive reinterpretation) as well as various major coping methods previously identified (i.e., problem and emotion-focused coping).

Studies have found that people generally use more than one particular type of coping strategy. In fact, combinations of coping strategies are often adopted to deal with different stressors during an individual's interaction with their environment (Goossens, Klein, \& van Achterbert, 2008; Tamres, Janicki, \& Helgeson, 2002). Kaba, Thompson and Burnard (2000) used a qualitative research methodology to explore the coping strategies of 42 heart transplant recipients. Results showed that recipients used a combination of eight coping strategies including acceptance/optimism, denial/avoidance, setting targets, comparing oneself with others, making attributions, seeking social support, having faith, and changing priorities and perceptions. Similar findings were noted with candidates for a lung transplant. Myaskovsky et al. (2003) interviewed 128 participants waiting for a lung transplant and found that they also used similar coping strategies as individuals who had received a heart transplant. Coping strategies used were active planning, seeking support, avoidance, acceptance, and self-blame.

However, studies also show that coping responses are not always similar among people with different chronic illnesses. For example, Goossens, der Klein, and van Achterberg (2008) reported that individuals with bipolar disorder differ in their coping strategies from that of the non-bipolar population. They studied 157 participants with stable bipolar disorder (free of either a manic or depressive episode four weeks prior to measurement) and found that strategies used by the participants were: active approach, palliative reaction (seeking distraction from thinking about a stressor), avoidance, seeking 
social support, passive reaction pattern (being totally overwhelmed), expression of emotion (irritation and anger at a stressor), and thinking reassuring thoughts. In comparison to two separate "normal" control groups, the coping patterns of individuals with bipolar disorder reflected a greater use of avoidance and passive reaction pattern strategies and less use of the active approach strategies in dealing with a stressor. Similar practices were also noted among people living with a perceived stigmatization related to their chronic disease. Peters, Apse, Blackford, McHugh, Michalic and Biesecker (2005) studied coping responses used by 174 adults with Marfan syndrome. Participants, who felt discriminated against or socially devalued for having Marfan syndrome, reported that they employed three common coping strategies: withdrawing from social situations (30\%), keeping their condition a secret (25\%), and educating others about Marfan syndrome (>50\%). Therefore, even if people use a similar combination of strategies for coping with a stressor, they may differ in the frequency with which they use each strategy. This finding leads to the question of whether or not there are determinants that make people choose certain coping strategies over the other types.

Indeed, research has affirmed that there are determining factors that influence the selection of certain types of coping strategies over others. According to Lazarus and Folkman (1984), three main factors influence coping appraisal: personality factors (e.g., selfesteem, locus of control), social support, and specific features of the situation. Positive interpretations are associated with choosing active and adaptive coping strategies (Bussing \& Fischer, 2009). Individuals with higher self-esteem, higher optimism, and a greater sense of control are more likely to select problem-solving strategies rather than denial or other avoidance coping strategies (Carver, Scheier, \& Weintraub, 1989; Holahan \& Moos, 1987). Holahan and Moos (1987) compared the coping strategies of a group of approximately 400 
community residents and over 400 persons entering treatment for unipolar depression. Results revealed that self-confident individuals were more likely to use active coping strategies and less likely to use avoidance coping strategies. Secondly, both the normal community residents and the psychiatric patients used both active and avoidance coping strategies in response to a stressor. Thirdly, while active coping strategies were used in reaction to both positive and negative stressors, avoidance coping was used only in reaction to negative stressors. Thus, it seems that the avoidance coping strategies are used in situations appraised as threatening and also when a person has limited personal and contextual resources. Yeh and Chou (2007) interviewed 2,642 persons receiving hemodialysis to study their coping patterns and found that an individual's choice of coping strategies was influenced by the types of stressor. Respondents were more likely to select emotion-focused coping strategies (e.g., worried, blamed self, thought it was someone else's problem), or strategies of seeking support, avoidance (e.g., smoked or ate more than usual), and thinking isolated thoughts (e.g., getting away from the problem, think of something else) to cope as the severity of stressors increased. The findings of the research described above are largely consistent with the assertions of Lazarus and Folkman (1984) who stated that problem-focused coping modes are more suitable for stressors perceived as controllable/ameliorative by action, whereas emotion-focused coping modes are more suitable for stressful situations that have to be accepted (i.e., diagnosis of cancer).

There are also demographic determinants of selecting coping strategies. Billings and Moos (1981) studied a group of 294 adult community residents and found statistically significant gender differences in coping responses. Results showed that women were more likely to use active-behavioral, avoidance, and emotion-focused coping strategies in 
comparison to men. Yeh and Chou (2007) also reported that men used more problemfocused as well as avoidance coping strategies. Level of education and income are also determinants of coping response. Individuals with higher socioeconomic status and income are more likely to use active-cognitive and problem-focused coping strategies (Billings \& Moos, 1981; Holahan \& Moos, 1987). One study also found that younger study participants use more mental disengaging and venting strategies to cope with a stressor than older participants (Fauerbach et al., 2002).

\section{Relationship between coping strategies and psychological distress.}

The use of various coping strategies has a variety of implications in terms of psychosocial outcomes (Collins, Baum, \& Singer, 1983; Rodrigue, Boggs, Weiner, \& Behen, 1993; Shen, Myers, McCreary, 2006). Although research has affirmed the existence of a significant relationship between coping strategies and psychological outcomes (i.e., depression, anxiety), the direction of causality is not conclusive due to the nature of many correlational studies used to generate the results. Nonetheless, evidence suggests that people with a certain mood show a propensity for selecting a particular coping strategy. For example, Coyne, Aldwin, and Lazarus (1981) reported that people with depression have a greater tendency to use more emotion coping, wishful thinking and avoidance coping, and are less likely to use problem-focused coping. Similarly, Veiel et al. (1992) also found that depressed individuals use at a higher frequency the coping strategies of distraction, avoidance, and negative appraisal.

There is also evidence to support the relationship of opposite causality; namely how certain types of coping styles are associated with different emotional outcomes. In general, 
strategies pertaining to problem-focused coping (e.g., planning, active coping and seeking social support) are positively associated with psychological adjustment, and negatively associated with psychological distress (Smith \& Wallston, 1992). In contrast, strategies of emotion focused coping such as venting of emotion, mental disengagement, and avoidance are associated with increased level of depression and psychological distress (Cronkite, Moos, Twohey, Cohen, \& Swindle, 1998; Penninx et al., 1998; Perez, 2009). For example, in a study involving 160 patients who were waiting for a lung transplantation, researchers found that the use of coping strategies such as harm appraisals, mental disengagement (i.e., psychological avoidance), seeking emotional support, and venting of emotions explained 52\% of unique variance in anxiety (Burker, Evon, Sedway, \& Egan, 2004). A similar finding is reported by Rodrigue, Boggs, Weiner, and Behen (1993) who examined mood and coping style of 51 candidates for bone marrow transplantation. They found that patients who use a more passive coping style (i.e., acceptance-resignation) were significantly more anxious and depressed. In theory, active coping strategies such as planning, taking action, and making the best of the situation may be perceived as adaptive, whereas strategies such as denial or disengaging may be perceived as maladaptive (Burker, Evon, Losielle, Finkel, \& Mill, 2005). These theoretical perspectives are affirmed with findings from various studies.

Doering, Dracup, Caldwell, Moser, Erickson, Fonarow, and Hamilton (2004) examined the relationship between three coping strategies (active-behavioral, activecognitive, and avoidance) and emotional distress in 84 patients with advanced heart failure. Results showed that the individuals who used the avoidance coping strategies more frequently were significantly more depressed, anxious, and angry. Avoidance coping was 
also negatively associated with vigour. Similar findings were also reported by researchers who studied recipients of heart transplantation. Dew et al. (1996) examined a group of 154 heart recipients one year after the transplantation. Data gathered via a standard clinical interview indicated that major depression was the most prevalent ailment affecting the recipients and that the use of the avoidance coping strategies was one of the major risk factors for depression. The relationship between coping pattern and psychological outcome was also explored among lung transplant candidates. Two separate groups of lung transplant candidates $(\mathrm{N}=128 ; \mathrm{N}=160)$ both used a similar variety of problem-focused and emotionfocused coping strategies: planning, active coping, positive reinterpretation, acceptance, disengagement, denial, and avoidance. Although used infrequently, an increased use of avoidance coping was most strongly and consistently related to quality of life and indirectly to depressive symptomatology (Myaskovsky et al., 2003)

A similar coping outcome was observed in people with other types of chronic disease. A meta-analytic review of study findings on the coping process of 3,133 men with prostate cancer revealed that they used a variety of coping methods including problemfocused, emotion-focused, and approach coping (e.g., positive reappraisal, seeking information). Men who used approach coping were found to experience positive emotions, whereas men who used avoidance coping experienced a heightened negative psychological adjustment (e.g., depression, distress, anxiety, etc.) (Roesch et al., 2005). A study of 50 gay men recently diagnosed with AIDS showed that the use of active-behavioral coping was inversely related to depression $(\mathrm{r}=-0.31, \mathrm{p}<0.05)$ and total mood disturbance $(\mathrm{r}=-0.45$, p<0.01) (Namir, Wolcott, Fawzy, \& Alumbaugh, 1987). In contrast, use of avoidance coping was related to an increased level of depression $(r=0.43, p<0.01)$. Results of various 
studies of coping process seem to support the premise of the stress and coping theory which states that even in a stressful situation, positive and negative effects can co-occur. Positive emotion, through the mechanism of infusing positive meaning into the situation, can buffer a negative effect (Folkman \& Moskowitz, 2000). Thus, individuals who actively choose to employ problem-focused coping strategies to positively reinterpret a stressful encounter, for example, may be able to avoid experiencing psychological distress.

People living with the visible physical signs of an ailment seem to exhibit similar coping patterns as people with other chronic diseases. For instance, Hill and Kennedy (2002) examined the impact of coping strategies on the psychological distress (i.e., depression and anxiety) of 89 people with psoriasis. The result was consistent to findings of people with cardiac diseases. In other words, using maladaptive coping strategies, particularly, venting emotions and mental disengagement, were significantly related to psychological distress. In addition, it appears that adopting more than one type of maladaptive coping strategy exerts a compounding effect. When a group of 78 individuals with visible scars from burn injuries were assessed for the effect of using more than one type of emotion-focused coping (i.e., venting emotions vs. mental disengagement), participants who used both types of strategies experienced higher level of depression than those participants who used only one of the two coping strategies (Fauerbach et al, 2002).

Findings from studies involving people with various chronic illnesses and physical conditions seem to collectively reveal a consistent pattern of psychological outcomes in relation to coping strategies. Maladaptive coping strategies such as avoidance and behavioral disengagement (avoidant-like coping) are significantly associated with 
psychological distress (i.e., depression and anxiety) (Burker, Evon, Sedway, \& Egan, 2004; Marsac, Funk, \& Nelson, 2006; Myaskovsky et al., 2003).

One of the controversial areas of coping research that has generated conflicting findings deals with the implication of using the strategy of denial coping. Evidence suggests that using denial is positively associated with psychological distress, but with a certain restriction. Denollet, Martens, Nykicek, Conraads, and Gelder (2008) conducted a ten-year prospective study examining the effect of repressive coping on cardiac prognosis in 731 individuals with coronary artery disease. The results showed that although the physiological outcome (mortality or non-fatal MI) of people who use repressive coping strategies is poor at ten years, their reported level of depression, anxiety, and anger at baseline was surprisingly lower than the people who use non-repressive coping. The authors attributed the difference in depression between two groups to the inability on the part of individuals with repressive coping to detect or report symptoms of depression. Similar findings were reported in another study. Levine et al. (1987) conducted a study involving 45 cardiac male patients hospitalized following an MI, of whom, 30 participants were followed for one year post-discharge. During hospitalization, men who used frequent denial coping strategies stayed less number of days in the critical care unit and experienced fewer cardiac problems in comparison to men who used denial coping strategies less frequently. However, at one year follow-up, men who used denial were less compliant with rehabilitation regimen and had a poorer recovery.

A meta-analysis examining the relative efficacy of avoidant and non-avoidant coping strategies also reported similar trends in the findings as the studies described above. Reviewers conceptualized avoidant coping as strategies of diverting attention away from either the source of one's reactions to the stressor, and attention coping as strategies of 
focusing attention on either the source, or one's reaction to the stressor. Results of the review indicated that in the short-term, avoidance coping was associated with positive adaptation to illnesses, but in the long-term, attention coping was associated with more positive outcomes (Suls \& Fletcher, 1985). It seems then, avoidance coping does offer some protective function on emotional distress as long as the intended benefit is short term. Avoidance may serve a beneficial function of relieving stress when a person is initially confronted with an overwhelming stressor for which one had not had time to "work through" and assimilate the experience. However, prolonged use of avoidance tactics may inhibit individuals from acquiring problem-solving behaviors necessary to deal with the stressor (Levine et al., 1987; Suls \& Fletcher, 1985).

The association between coping styles and psychological distress has an important relevance for studying the adjustment of individuals living with CHD. Research has shown that people with CHD use a variety of coping behaviors similar to those used by people with different chronic illnesses including strategies of, active coping (e.g., learning about the disease), planning (e.g., anticipating one's reaction to his/her environment, dealing with one thing at a time), practicing positive reinterpretation (e.g., thinking "I was chosen for a purpose and am here for a reason"), accepting the situation, mentally disengaging (e.g., pushing boundaries by climbing, parachuting, drinking alcohol), employing restraint coping (e.g., hiding scars), denying the situation, and suppressing feeling of being different. Although information is available about different strategies used by people with CHD, no research has been conducted to examine the relationship between various coping strategies and psychological distress in this group, nor coping strategies' impact on psychological outcome. This type of knowledge would enable nurse practitioners to identify those people 
with congenital heart disease at risk for experiencing emotional distress and to develop appropriate interventions targeted at alleviating risk factors.

\section{Social support}

The effect of social support has long been the focus of health research (Cohen et al., 1985; Stansfeld, Fuhrer, \& Shipley, 1998). Researchers, however, have not attained consensus on the definition of social support or how best to measure the construct. As a result, the phrase social support has been loosely used to denote a wide variety of phenomena that characterizes the social environment (Helgeson, 2003). The definition of social support may encompass the actual supportive behaviors exchanged between people, or the early childhood experiences that may influence one's perception about the likelihood that someone will be supportive (Uchino, 2004). Cohen and Syme (1985) define social support as simply the resources provided by other people. Social support functions on the premise that the recipient of the support will be led to believe that he or she is a valuable person worthy of love and esteem, and is connected to a social network of communication.

The study of social support can be examined primarily from three different theoretical perspectives: 1) stress and coping perspective, 2) social constructionist, and 3) relationship (Lakey \& Cohen, 2000). The stress and coping perspective claims that the major benefit of social support is achieved through protecting individuals from the harmful effects of stress. The approach of the stress and coping perspective is aligned with the theory of Transaction Model of Stress and Coping by Lazarus and Folkman (1984). In the social constructionist perspective, the effect of social support is achieved by the enhancement of one's identity and self-esteem, irrespective of the level of stress. Two of the theories that 
resonate with the social constructionist perspective are the theories of social-cognition and symbolic interactionism. In the relationship perspective, the effects of social support are manifested through relationship processes in which the support occurs, including companionship, intimacy and low social conflict. Although social support is examined through different theoretical perspectives, the current study is heavily guided by the stress and coping perspective. From the context of the stress and coping approach, social support offers protection from the stressor's adverse effects by promoting coping behaviors and altering the appraisal of the stressor.

Study findings have consistently demonstrated that significant relationships exist between social support and biopsychosocial outcomes (Case, Moss, Case, McDermitt, \& Aberly, 1992; Schulz \& Decker, 1985; Siegal, Calsyn, \& Cuddihee, 1987). In situations involving a stressful experience, such as learning to live with a chronic physical illness, social support can influence the outcome. In terms of physiological outcomes, decreased levels of social support have been linked to an increased risk of mortality. For example, in a prospective cohort study of 430 persons following coronary angiography, low levels of social support (operationalized as fewer than three people of a close relationship), were associated with 2.4 relative risk of mortality (Brummet, Barefoot, \& Siegler, 2001).

\section{Different measures of social support.}

Studies have affirmed that social support is indeed a multidimensional construct (Oxman, Freeman, Manheimer, \& Stukel, 1994). Researchers commonly distinguish social support using structural and functional dimensions. Structural support represents the objective characteristics of a social network such as existence, and the relations among network members, i.e., marital status, number of friends, frequency of interaction with 
friends, etc. (Cohen \& Syme, 1985; Lyyra \& Heikkinen, 2006). Functional support indicates the specific functions/resources that social relationships provide. Functional supports are qualitative measures that generally include three types of support: emotional, instrumental, and informational (Helgeson, 2003). Emotional support refers to the sense of being heard, valued, loved, and accepted. Instrumental support, also known as tangible support, represents tangible assistance provided in the form of direct material aids. Information support denotes the provision of advice or guidance. Some researchers add an additional function of belonging support, which reflects the existence of people with whom to engage in social activities (Uchino, 2004). Functional support is further categorized along two dimensions: perceived available support and actual received support (Kessler, 1992; Uchino, 2004). Perceived social support indicates the individual's perception of availability of support and/or satisfaction with support provided. Received social support reflects the specific supportive behaviors provided to the recipient (Haber, Cohen, Lucas, \& Baltes, 2007). Researchers claim that perceived and received social supports are not highly related and in fact, each support is linked to different health outcomes. Of the perceived- and received social supports, the research has been more extensive and findings more confirmative in relation to perceived support. Results from different studies demonstrate that perceived support is inversely correlated with symptomatology (Cohen, Underwood, Gottlieb, 2000). Thus, the perception of social support availability is associated with a positive emotional adjustment to stress (Cohen \& Wills, 1985; Kessler, 1992). Results were not as confirmative in relation to received social support. For example, Lepore, Glaser, and Roberts (2008) conducted a longitudinal study to examine the relationship between received social support and psychological distress in 71 women with breast cancer. The data were 
collected at two points in time: three months and 18 months post diagnosis. They found that received social support at $\mathrm{T} 1$ was positively correlated with negative affect at $\mathrm{T} 2$. The authors explained that the recipients may interpret received social support as a sign of personal incompetence, which in turn, can undermine the recipient's self-esteem. A similar result is reported in a study by Maisel and Gable (2009) who surveyed 67 cohabiting couples. Participants were asked to complete a daily questionnaire regarding the nature of their interaction with their respective partners for a period of two weeks. The results showed that visible support (both the participant and the partner are aware of support received and given, respectively) tended to be associated with negative affect (i.e., hopelessness, anxiety). However, such a finding was limited to situations in which the recipient did not perceive the support provider to be understanding or caring about the recipient's stressor.

Penninx et al. (1998) identified three elements of social support: social network, type of social support, and satisfaction of social support. Social network, representing the structural aspect, refers to the number and frequency of contact. The type of social support, which denotes the functional aspect, consists of informational support, instrumental support, and emotional support. Satisfaction of social support, which also pertains to the functional aspect, deals with the perceived adequacy of the support given. Social support has been linked to different psychological outcomes, such as a sense of well-being (Cohen \& Syme, 1985; House, 1981; Kessler \& McLeod, 1985; Turner, 1983). Oxman, Freeman, Manheimer, and Stukel (1994) studied social support and depression in 155 elderly patients six months after cardiac surgery. The three aspects of social support measured were social network, type and amount of support, and perceived adequacy of support. The final model was produced using a multiple regression analysis and adjusted for the following covariates known to 
influence depression: pre-surgery depression score, mental health treatment, history of myocardial infarction, and impairment in activities of daily living (ADL). The result indicated that the only social support indicator that was significantly related to a decreased level of depression at the six month mark was that of social network, meaning the total number of emotionally close network members $(p=0.010)$. However, the favourable effect of a social network on psychological outcome is not consistently observed. In fact, Penninx et al. (1998) found that having diffuse relationships (with neighbours, friends, acquaintances, collegues, and others) exerted the negative effect of increasing the level of depression.

Frasure-Smith et al., (2000) studied the interrelationship between baseline depression and social support in terms of changes in depression symptoms in a group of 887 individuals over the first year post-myocardial infarction. The aspect of social support measured pertained primarily to an individual's social network. The result indicated that the effect of social support was found only in those who had baseline depression. In other words, the effect of social support was not significant for individuals who were not depressed at the baseline. However, for individuals who were depressed at the baseline (Beck Depression Inventory score $>10$ ), the worsening of depressive symptoms one year later was related to the least amount of social support. More specifically, individuals with a perceived low level of social support; no relatives whom they considered close; lower number of regular interactions with friends and relatives whom they considered close; and who lived alone demonstrated more depressive symptoms at one year after an MI. A similar buffer effect of social support is reported by others. The moderator (buffer) effect of perceived social support was examined in a study involving 1,366 women from a community (Kinsinger, McGregor, \& Bowen, 2009). The protective role of social support on the relationship 
between perceived breast cancer risk and emotional distress (i.e., depression, anxiety) was specifically studied. The findings showed that an increased level of perceived breast cancer risk was associated with emotional distress, but only among women with low perceived social support. Particularly, when women were recipients of tangible social support and positive social interaction (e.g., "have a good time with"), the level of anxiety was attenuated. Penninx et al. (1998) reported similar findings. Effects of social support on depressive symptoms were examined in 2,810 persons with five different types of chronic diseases: diabetes mellitus, lung disease, cardiac disease, arthritis, and cancer. A normative sample of 719 persons without any chronic disease served as the control to which the outcome variable of depression could be compared. The results demonstrated both the main effect and the interaction effect of social support. The main effect was detected because irrespective of the presence of a chronic disease, having a significant other and many close relationships was associated with a lower level of depression scores. The interaction effect of social support was also present. The decrease in a depressive score was found in individuals who were recipients of emotional support, however the effect was observed only in people with cardiac disease or arthritis ( $\beta \mathrm{s}=-0.13$, and -0.12 , respectively). The effect was also not evident in healthy participants without any chronic disease.

Social support is a multifaceted construct and as such, it is important to consider different dimensions of social support rather than using a combined score (a single undifferentiated global measure) to assess and examine the impact on psychosocial outcome. And yet, it was noted during the literature review for this study that researchers do not consistently report on the specific aspect of support or how it is being measured. As a result, without the knowledge of the specific dimension of social support being measured, the 
validity of the study findings may be questionable. Nonetheless, based on study findings that did provide specific dimensions of social support, it is clear that the functional indicators of social support (emotional, instrumental, and informational support) are linked to emotional outcome. For example, respondents who receive emotional support from a close person were found to experience better mental health in comparison to those who did not receive emotional support (Brown, Andrews, Harris, Adler, \& Bridge, 1986). Yu, Lee, Woo, and Thompson (2004) also reported a similar finding in their study involving 227 patients with congestive heart failure admitted to a hospital. High levels of psychological distress, in particular, depression were observed in patients who perceived the given emotional and informational supports as unsatisfactory. Although Yu and her colleagues did not differentiate between emotional and informational supports as is typically done (Cohen, 1985), nonetheless the findings reflect the significant impact the functional aspect of social support exerts on psychological outcome. In a prospective study involving 212 recipients of hematopoietic stem cell transplantation, Wells, Booth-Jones, \& Jacobsen (2009) examined whether the variables of coping and social support predicted anxiety and depression in the recipients. Data were collected both at pre- and six months post-transplantation. The result of the study showed that tangible social support was negatively correlated with posttransplantation depression, whereas tangible, appraisal, and belonging support were negatively correlated with post-transplantation anxiety.

In terms of the relationship between the evaluation of social support and the emotional outcome, Hann et al. (2002) found that increased perceived adequacy of social support was significantly related to fewer depressive symptoms in a sample of 342 people 
with cancer. Holahan and Moos (1981) reported that the perceived quality of family support was negatively related to depression in a community sample of 593 people.

Evidence indicates that social support also exerts an influence via main or interaction effects (buffer effect). The premise of the main effect model is that the increase in positive psychosocial outcome associated with social support occurs irrespective of the presence of stress (Broadhead et al, 1983; Penninx et al., 1998). On the other hand, the buffer effect of social support arises only in situations the individual appraises as stressful and in which one's coping resources are exceeded (Folkman \& Lazarus, 1985). Social support functions as a buffer between the stressful event and the emotional response by attenuating the appraisal (secondary appraisal) of the stress. In other words, social support enables the recipient to redefine the stressful encounter in such a way that enhances his or her perceived ability to cope with the stressor, and thus, attenuate the level of responsive emotional distress (i.e., helpless-related depression) (Cohen \& Willis, 1985).

Receiving different forms of social support is not always associated with favourable outcomes, however. Studies have indicated that the various social supports provided need to be commensurate to both the stressful situation as well as to the specific need of the recipient. For example, Penninx et al. (1998) examined the coping strategies of people with different chronic illnesses, e.g., diabetes mellitus, lung disease, cardiac disease, etc. Although the results indicated the favourable effect of various social supports for groups, instrumental support had a negative effect for a selected group of people. Receiving instrumental support had a positive relationship with depression in people with diabetes mellitus. In other words, for individuals with diabetes mellitus, receiving instrumental social support was associated 
with an increased level of depression. The finding, which was also observed in a healthy control group, was limited to only the group of diabetic individuals.

Furthermore, researchers are starting to identify that both support and conflict coexist in many social relationships and that both aspects of a relationship exert effects on emotional distress. Holahan, Moos, Holahan, \& Brennan (1997) examined in a four-year longitudinal study the social support, coping and depressive symptoms of 183 cardiac patients. The data were collected at two points (Time 1 and Time 2) at four years apart. The findings of the study revealed that stressful social support was related to an increased level of depression. In contrast, positive social support was related to a decreased level of depression. Research on social support in the past decade has primarily been conducted based on the assumption of the positive effect of social support on well-being of the individuals. However, some researchers claim that interpersonal relationships encompass both supportive dimensions and potentially conflictual or burdensome dimensions (Cohen, Underwood, Gottlieb, 2000). In other words, social relationships consist of both costs and rewards. In addition, sometimes the person who provides support could at the same time become a support burden (Helgeson, 2003). Thus, social interactions consisting of a high level of social support may occasionally bring on disputes, embarrassment, envy, or invasion of privacy. Helgeson claims that negative interactions and positive interactions are independent constructs that contribute to different outcomes. Researchers also argue that social relationships characterized by negative or conflict-laden interactions are better predictors of a depressed mood than supportive interactions (Schuster, Kessler, \& Aseltine, Jr., 1990). Both the positive and the negative aspects of received social support make independent contributions to the psychosocial outcome. Helgeson further asserts that 
negative interactions exert a stronger effect on people's emotional state as their occurrences are salient and unexpected. Helgeson's hypothesis about the significant impact of negative interaction is supported in a study by Rook (1984) of 120 widowed women. The purpose of her study was to examine the relative impact of both positive and negative social interaction on the well-being of the participants. The results showed that the number of problematic social ties (i.e., invaded privacy, being taken advantage of, consistently provoked conflicts) was significantly related to a decreased level of well-being. In fact, while both the positive and negative social relations affected the well-being of the participants, the negative social relations exhibited a greater impact on well-being. A similar finding is reported by Abbey, Abramis, and Caplan (1985) who studied the relationship between social support and social conflict in 168 undergraduate students. They found that the greater the amount of social conflict from people in the personal lives of the participants, the greater the level of participant anxiety, depression, and a poor quality of life. Based on findings from studies described above, the effect of social support on psychological distress of people living with various health challenges should be examined from both positive and negative aspects of social relationships.

Evidence shows that a large number of young adults with CHD live at home, in part due to their physical illness, under what is sometimes perceived as an "overprotective" parental rearing practice (Kovacs, Sears, \& Saidi, 2005; Wright, Jarvis, Wannamaker, \& Cook, 1985). For these young adults who may be facing the developmental tasks of gaining independence and establishing self-identity, instrumental support may not be the type of support they particularly desire nor find helpful (Claessens et al., 2005). It is plausible to 
assume that undesired social support may in fact increase one's sense of inadequacy and helplessness, and exert a negative influence rather than produce a positive effect.

Social support can have a significant relationship with psychological distress in people with CHD. For example, in an anecdotal situation where a young male adult living with CHD described being left out of playing soccer with friends, having a close friend who chose to not play soccer and instead offer emotional support may have attenuated the emotional distress of feeling abandoned. Likewise, a person living with CHD who is selfconscious of his or her body might find having an intimate partner who offers emotional support provides a feeling of worth and prevents the onset of depression. Alternatively, it is also plausible that social support promotes adaptive coping patterns in a person with CHD, which in turn results in a positive effect (mediation effect). With accumulating evidence on the positive influence of social support on psychological distress, it would be well justified to explore the important role it plays in the adjustment of people with CHD.

\section{Summary}

In summary, an examination of the literature clearly indicates that certain psychosocial variables, particularly, types of social support and coping strategies are closely associated with emotional distress in people with various chronic health challenges. In the context of people with CHD, however, very little is known about the psychosocial adjustment of this unique group of people. Furthermore, it is also unclear whether the factors found to influence emotional distress in people with a different type of illness also yield a similar outcome in people with CHD. Therefore, this study will examine the influence of social support and coping strategies on psychological distress among adults living with CHD. 


\section{Conceptual Framework}

The purpose of this study was to examine the relationship between selected psychosocial variables and psychological distress in people living with CHD. The variables

- types of social support and different coping strategies - were chosen based on the findings from the literaure. The conceptual framework illustrated in Figure 1 focuses on two aspects: the relationship between psychosocial predictor variables (types of social support and coping strategies) and the outcome variable (psychological distress); and the relationships between psychosocial variables themselves. In the following section, the hypothesized relationships among study variables are discussed.

\section{Psychological distress (anxiety and depression).}

Although many individuals with CHD who survive into adulthood following medical intervention (i.e., surgical repair, catheter intervention) are reported to be leading productive lives free from psychological distress, study findings indicate that there is a group of people who experience varying degrees of psychological challenges related to their heart disease.

"The friends that I had left me behind, because they knew that I wasn't able to follow them... that was a period that I didn't want to live anymore. It was hard for me to accept that my friends went to play football, which is a very intensive sport, so...they thought, 'he isn't able to do that'... and that was very difficult for me." (Claessens et al., 2005, p. 6) 
Figure 1. Conceptual Framework

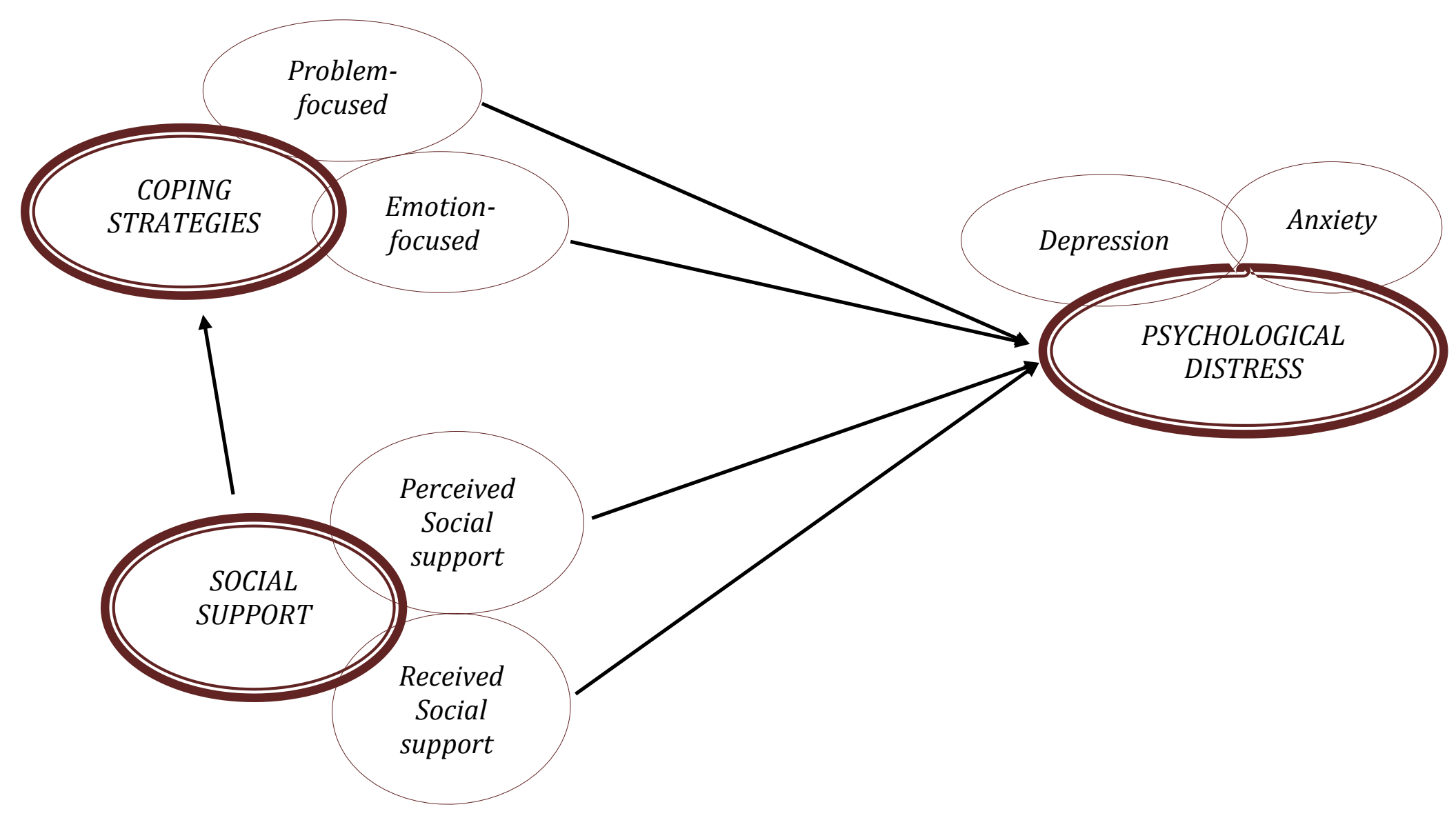


One of the psychological challenges study participants reported is a feeling of emotional distress (e.g., depression, anxiety) arising from their perception of being "different" from their peers and the general population. Depression is noted in a significant number of individuals with CHD, many of whom do not display observable signs or behaviors typically associated with psychological distress. Depression, whether the symptoms are discernable or not, can have dire physiological consequences, i.e., increased mortality (Frasure-Smith et al., 2000), and therefore, is an outcome worthy of examination particularly in people experiencing chronic cardiac diseases. It is imperative that nurses have the ability to identify people with CHD who are at risk of developing depression and promptly intervene. This will not only enhance the quality of life for people living with congenital heart disease and help prevent premature deaths, but it will also reduce health care costs.

\section{Relationships between psychological distress and social support.}

As indicated previously, the outcome variable of psychological distress in the conceptual model included depression and anxiety. The effect of social support on health outcomes, particularly emotional distress (e.g., depression, anxiety), has been extensively documented in literature. Research indicates that social support is a multidimensional construct where different aspects of support relate differently to the observed outcome. For example, received support is linked to a different outcome in comparison to that of perceived support. Failure to receive support (i.e., emotional support) during a stressful period from a person of close relationship (Brown, Andrews, Harris, Adler, \& Bridge, 1986), or dissatisfaction with received support have been linked to increased level of depression (Holahan \& Moos, 1981). 
Evidence suggests that social support is also related to use of coping strategies. People who receive social support are more likely to use an adaptive coping strategy in comparison to those who do not. In contrast, people who receive an inadequate level of social support tend to use avoidance coping strategies, which in turn, is related to an increased level of psychological distress. In this study, two types of social support are measured.

\section{Relationship between coping strategies and psychological distress.}

Research has identified coping as one of the most significant variables associated with psychological distress in people with physical illnesses. The proposed examination of coping as a predictor of psychological distress in people living with CHD is informed by the stress and coping theory proposed by Lazarus and Folkman (1984). For people living with CHD, stress manifests as they struggle to deal with issues related to managing a chronic health challenge. According to the stress and coping theory, stress is a situation that a person appraises as taxing or exceeding his or her resources (Lazarus \& Folkman, 1985). The cardinal concepts in the stress and coping theory are: cognitive appraisal and coping. Cognitive appraisal, which refers to a person's subjective analysis of an event, divides into primary and secondary appraisals. Primary appraisal is the process through which a person attempts to determine whether an encounter is irrelevant, benign-positive, or stressful (Peacock \& Wong, 1993). When an encounter is perceived as taxing or surpassing one's coping resources, a person judges the encounter as posing a threat or a challenge. The appraisal of a threat is made when the potential for harm or loss exists, whereas the appraisal of challenge is made when the situation poses potential for growth, mastery, or gain. 
Secondary appraisal represents the process by which an individual evaluates his or her available coping resources and options for managing a stressful situation.

Coping denotes the person's cognitive and behavioral attempts to manage a particular stressful person-environment relationship. Coping efforts involve two major methods: problem-focused coping and emotion-focused coping. Problem-focused coping involves strategies directed at changing or attenuating the problem causing the distress, while emotion-focused coping involves strategies that are aimed at regulating distressing emotions. Folkman and Lazarus (1985) reported that problem-focused coping may be more effective in situations appraised as changeable or controllable, whereas emotion-focused coping may be more effective in situations appraised as unchangeable. Numerous studies have demonstrated that certain types of coping strategies are associated with psychological distress. Burker, Evon, Sedway, and Egan (2004) reported that use of coping strategies such as avoidance and behavioral disengagement are significantly related to depression and psychological distress. In addition, people who use emotion-focused coping more frequently than problem-focused coping experience a higher level of depression than those who use problem-focused coping more frequently than emotion-focused coping (Coolidge, Segal, Hook, \& Stewart, 2000; Cronkite, Moos, Cohen, \& Swindle, 1998).

In the conceptual framework (Figure 1), coping is conceptualized as consisting of problem-focused and emotion-focused coping. As discussed in the literature review, although research shows, in general, a positive relationship between coping and psychological outcome, a closer examination reveals that different types of coping strategies relate differently to psychological outcome. For example, the use of avoidance coping is 
often linked to an increased level of emotional distress, whereas problem-focused coping is often linked to a decreased level of emotional distress.

\section{Relationships between social support and coping.}

Research indicates that social support is related to the use of coping strategies. Although the direction of the relationship between social support and coping strategies is debated, the evidence more consistently supports that coping strategies mediate the relationship between social support and psychological distress. That is, the dominant direction of relationship described in the literature favors the direction from social support to coping strategies (Cohen, Underwood, Gottlieb, 2000; Holahan, Moos, Holahan, \& Brennan, 1995; Holahan, Moos, Holahan, \& Brennan, 1997). People who receive social support are more likely to use adaptive coping strategies in comparison to those who did not. Researchers are starting to identify that both support and conflict coexist in many social relationships and that these aspects of a relationship affect psychological distress. Holahan, Moos, Holahan, and Brennan (1997) conducted a four-year longitudinal study examining the social support, coping and depressive symptoms of 183 cardiac patients. The data were collected at two points over four years apart. The premise of the study was that negative aspects of a relationship can undermine one's ability to cope. Social context was assessed with the support or stressors from family or extra-family, and coping was assessed with adaptive coping (positive reappraisal and problem-solving coping strategies). Results of the study indicated that both positive and negative social supports are indirectly related to the level of depression of the cardiac patient through coping. In other words, stressful social support is related to an increased level of depression mediated by a decreased level of 
adaptive coping strategies (i.e., problem-focused coping). In contrast, positive social support is related to an increased level of adaptive coping, which in turn in related to a decreased level of depression.

Evidence suggests that the direction of the relationship between social support and coping could exist in the opposite direction. In other words, coping strategies could influence the type of social support, which in turn, could influence the psychological outcome. For example, Dunkel-Schetter, Folkman, \& Lazarus (1987) investigated the stress and coping of 150 middle-aged community residents. The results of their study revealed that problem-focused coping was significantly associated with an increased level of informational support, whereas emotion-focused coping was significantly associated with a decreased level of informational support.

In summary, research findings indicate that types of social support and different coping strategies have a significant relationship with psychological distress in people with various types of chronic health challenges. More specifically, evidence suggests that the type of social support influences the type of coping strategies used by individuals, which in turn, influence psychological outcomes (Holahan, Moos, Holahan, \& Brennan, 1995; Holzman, Newth, \& DeLongis, 2004; Kim, Han, Shaw, McTavish, \& Gustafson, 2010; Manne, Pape, Taylor, \& Dougherty, 1999). While the direction of the relationship between social support and coping could be manifested in a reverse direction (e.g., some studies have found that types of coping strategies influence types of social support, which in turn, influence psychological outcomes [Dunkel-Schetter, Folkman, \& Lazarus, 1987; Wortman, 1984] ), the evidence more fully supports the hypothesis that coping strategies mediate the relationship between social support and psychological distress. The hypothesized conceptual 
model suggests that the types of social support (perceived and received social support) influence the types of coping strategies (problem-focused, wishful-thinking, and/or denial/avoidance) used by individuals, which in turn, inflences the psychological distress (anxiety and depression) of individuals living with CHD. Implied in this model are a number of relationships:

- Perceived social support is directly related to anxiety/depression.

- The effect of perceived social support on anxiety/depression is partially mediated by coping strategies of problem-solving.

- The effect of perceived social support on anxiety/depression is partially mediated by coping strategies of wishful thinking.

- The effect of perceived social support on anxiety/depression is partially mediated by coping strategies of denial/avoidance.

- Received social support is directly related to anxiety/depression.

- The effect of received social support on anxiety/depression is partially mediated by coping strategies of problem-solving.

- The effect of received social support on anxiety/depression is partially mediated by coping strategies of wishful thinking.

- The effect of received social support on anxiety/depression is partially mediated by coping strategies of denial/avoidance. 


\section{Chapter 3}

\section{Methods}

This study used a cross-sectional survey design in which the data were collected using self-report measures. Additional information (i.e., date of birth, diagnosis, etc.) was obtained from participants' individual medical charts.

\section{The Sample}

\section{Sample size.}

The determination of sample size in structural equation modeling (SEM) has long been a vexing question for researchers that has resulted in a number of recommendations put forth by different authors. Two primary approaches are reflected in the literature. The first approach involves estimating observations per variable. A traditional "rule of thumb" in multivariate statistics dictates 10 to 20 subjects per variable (Schumacker \& Lomax, 2004). The second approach, the "rule of 10', is one of the most widely regarded guidelines for determining the sample size. The rule of 10 refers to choosing 10 observations per indicator, which was originally suggested by Nunnally (1978) who stated "a good rule is to have at least ten times as many subjects as variables". Others have suggested a rule of 5 , meaning 5 observations per indicator (Bentler \& Chou, 1987). Still, some researchers are in consensus that 100 to 150 participants is the minimum required sample size for conducting structural equation model (Ding, Velicer, \& Harlow, 1995).

The determination of sample size for this study was guided by the recommendations of Garver and Mentzer (1999) and Hoelter (1983) who proposed a "critical sample size" of 
200. This recommendation resonates with Kenny's (2011) statement, " 200 is seen as a goal for SEM research". The premise of their recommendation is that a sample size of, minimum, 200 participants would provide sufficient statistical power for data analysis. A final factor that guides the determination of sample size is feasibility; at times there may be incomplete or unusable data. The decision was made to obtain a sample greater than the minimum suggested by Kenny. Based on the Adult Congenital Heart Disease (ACHD) clinic population and available resources, it was determined that a sample of 250-300 was a reasonable goal.

\section{Selection process.}

A convenience sample was drawn from the (ACHD) program clinic at a large tertiary care hospital in Vancouver, British Columbia (BC). Inclusion criteria for the study were: 19 years of age or older with CHD; resides in BC; able to speak, read and comprehend instructions of self-report instruments in English; and consent to participate in the study. No restriction to upper age limit was applied so long as the person satisfied all the remaining inclusion criteria. Exclusion criteria in sample selection included: concurrent major illness (e.g., cancer); concurrent major psychiatric illness; inability to speak or write English; cognitive inability to comprehend instructions or items in self-report measures (e.g., trisomy 21); or residence outside of BC.

From September 2, 2010 to May 30, 2011, a total of 1,151 eligible participants were approached to participate in the study. The potential participants were contacted using two different strategies. The first strategy involved approaching the subjects while they were waiting for their scheduled appointments at the ACHD clinic at a large Acute Care Hospital 
(Figure 2). The second strategy involved the use of a mail-out questionnaire with an accompanying letter that described the study (Figure 3). The method of mail-out questionnaires was chosen in order to reach those participants who may be in-between their regular check-ups during the period of the data collection and thus, would not have had an opportunity to be asked to participate in the study. Of the total 1,151 people approached to participate in the study, 551 were from the ACHD clinic. Of these, 131 people refused to participate in the study for the following reasons: too busy $(n=24)$, not interested $(n=38)$, perceived inability to be of any help $(n=27)$, or did not offer a reason $(n=42)$. Of the 377 individuals who agreed to participate, 154 completed the questionnaire while at the clinic, and 144 chose to take the questionnaire home to complete it and return by mail. Ten people, who had initially expressed interest in participating in the study, were lost to recruitment as they left immediately after their scheduled appointments before the researcher had a chance to discuss the study with them. Of the 144 individuals who took home the questionnaire, 32 participants returned the completed questionnaire while 112 participants did not. After a period of five months, a reminder letter to return the questionnaire was sent to the nonresponding participants who took the questionnaire home. Three participants returned the completed questionnaire following the reminder letter. In summary, a total of 189 participants recruited from the direct contact at the ACHD clinic submitted the completed questionnaires. Of 189 completed questionnaires, five were excluded due to incomplete data, resulting in the sample size of 184 .

With respect to the use of the mail-out questionnaire to reach potential participants, a total of 600 questionnaires were mailed with the cover letter describing the study and the 
Figure 2. Selection Process Through Direct Contact at the ACHD Clinic

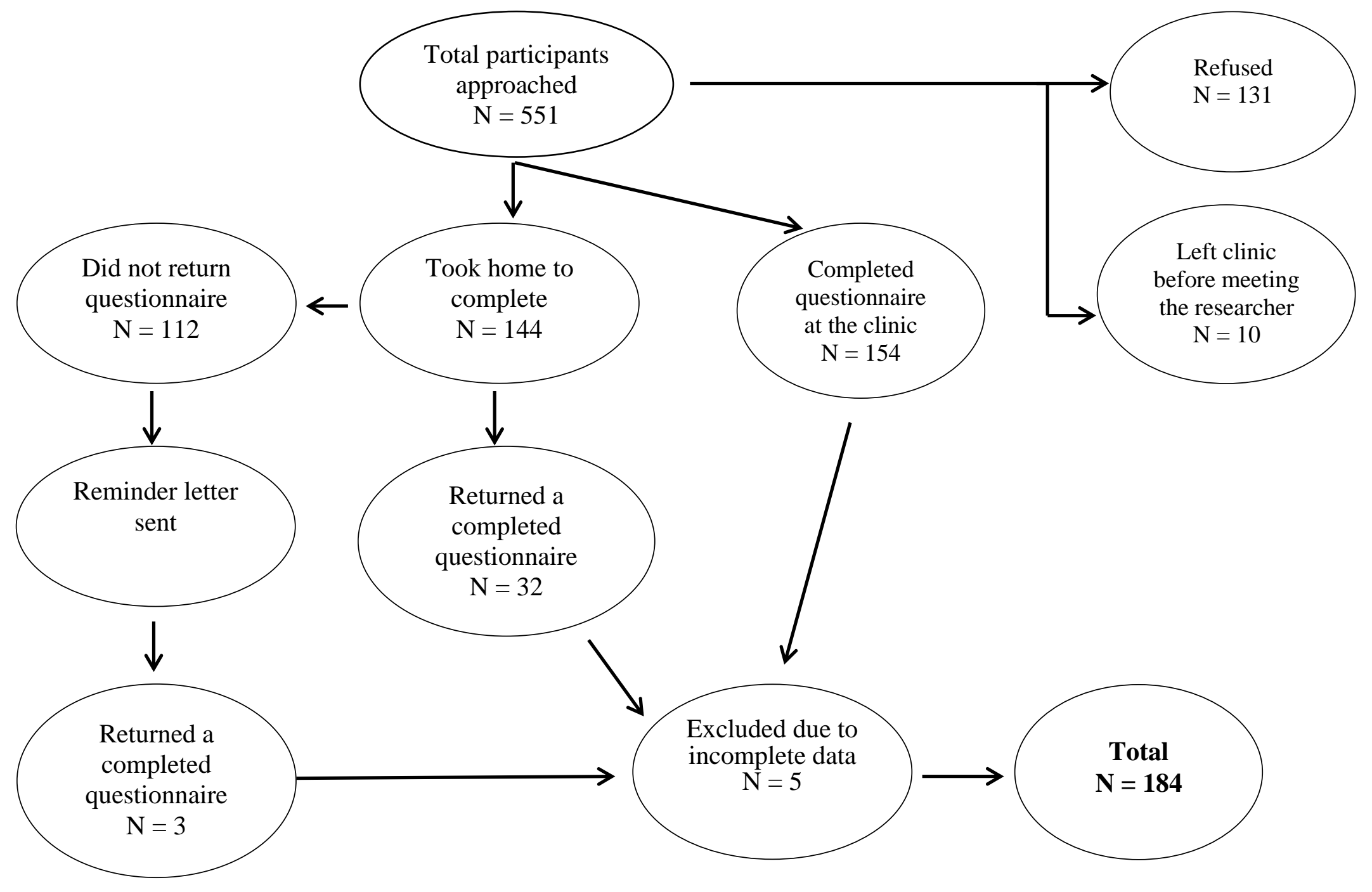


Figure 3. Selection Process Through Mail-out Questionnaire

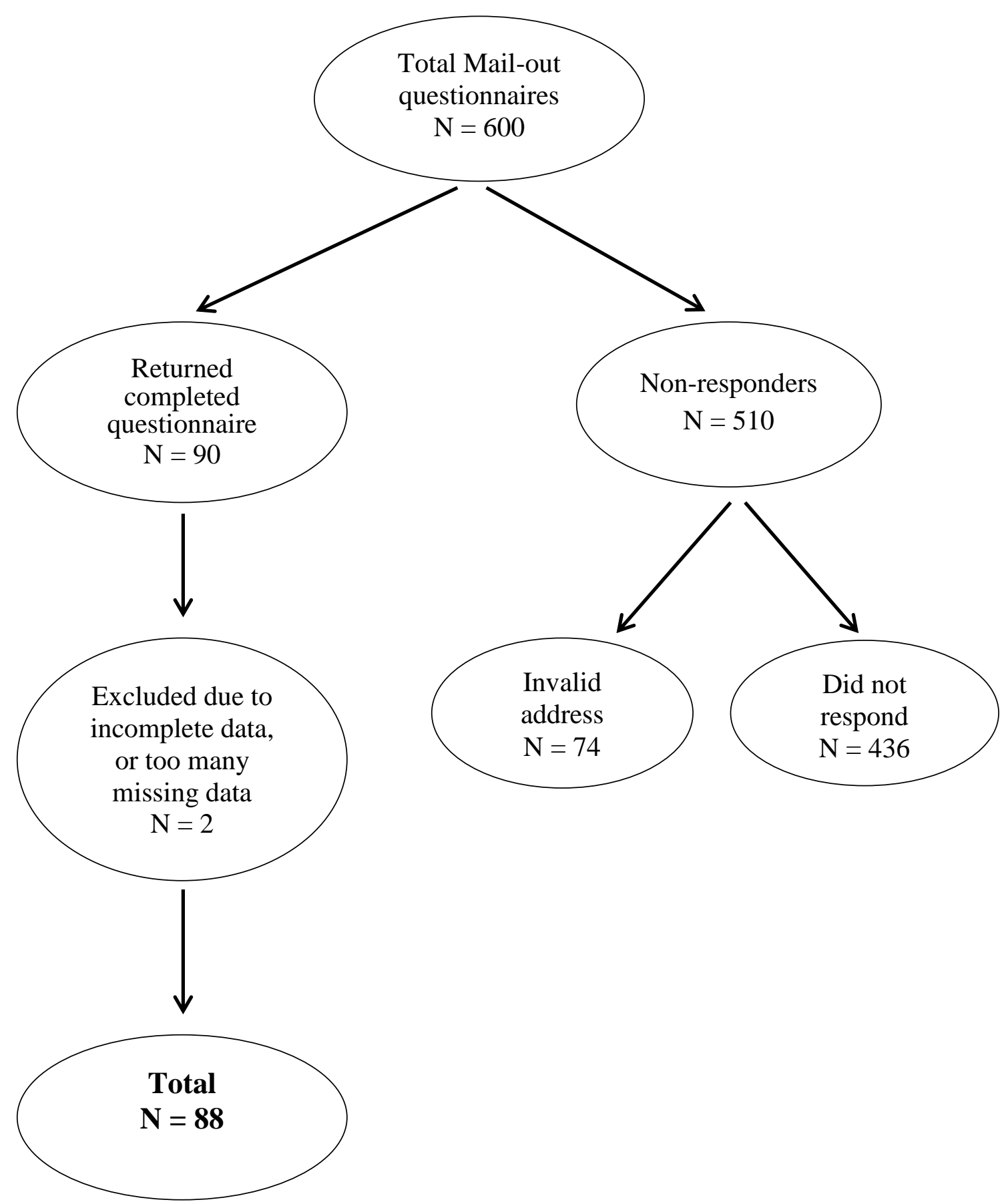


consent form. Ninety individuals responded and returned the completed questionnaire. However, two were excluded due to significant missing data, resulting in the total sample of 88. Of the remaining people, 436 individuals did not respond and 74 questionnaire packages were returned due to an invalid address. There were no significant differences between the responders and the non-responders of the study with respect to age: $\mathrm{M}$ (responders) $=36.79$, $\mathrm{SD}=14.76$ and $\mathrm{M}$ (non-responders) $=35.64, \mathrm{SD}=13.50, \mathrm{t}(920)=1.15, p=0.250$, and gender $\chi 2(1)=0.557, p=0.456$. The final sample size, from a combination of $184(36 \%)$ from the ACHD clinic and 88 (14.6\%) from the mail out questionnaires, was 272 participants.

\section{Instruments}

The following section presents a general description of the instruments that were used to measure the key study concepts. A more detailed discussion about the psychometric properties of each instrument is provided later in the results section. The complete sets of items used in the questionnaires are presented in Appendix B.

\section{Anxiety.}

The Beck Anxiety Inventory (BAI) was used to assess the level of anxiety in individuals with CHD (Beck, Epstein, Brown, \& Steer, 1988). The BAI is widely used to measure the severity of anxiety. The BAI is a self-report measure that consists of 21 -items and responses are rated on a 4-point Likert-type scale that ranges from not at all (0) to severely-it bothers me a lot (3). The BAI is designed to discriminate anxiety from depression in a psychiatric population. The range of total score is 0 to 63 , in which a score between 0 21 indicates very low anxiety, 22-35 moderate anxiety and over 36 indicates severe anxiety. 
Internal consistency reliability established with a sample of psychiatric patients is 0.92 and one week test-retest reliability is 0.75 (Beck et al., 1988). Discriminant validity has been demonstrated from the comparison of patients with primary DSM-III anxiety disorder and patients with primary DSM-III depression. The mean BAI score is significantly higher in the anxious group in comparison with the depressed group. Concurrent validity was established with the Hamilton Anxiety Rating Scale-Revised ( $\mathrm{r}=0.51)$ and the Cognition ChecklistAnxiety subscale (r=0.51).

The scale was developed by Beck and his colleagues in an attempt to address the problem of the relatively high correlation $(r>0.50)$ between anxiety and depression often observed in self-report instruments (Beck, Epstein, Brown \& Steer, 1988). Although the high correlation may arise from a genuinely shared symptomatology between the two constructs, it may also be related to a lack of discriminant validity of the instruments. The purpose of the BAI was to reliably discriminate anxiety and depression while maintaining the convergent validity (Beck et al., 1988). Each of the 21-items in the BAI describes a common symptom of anxiety. The BAI has been used in different patient groups including high school students and psychiatric inpatient study samples (Osman et al., 2002).

The factor structure of the BAI has been examined in a number of different studies and a varied number of factors ranging from two to five are reported. Beck et al. (1988) reported a two factor structure consisting of somatic symptoms and subjective and anxiety symptoms in a sample of 1,086 psychiatric outpatients. Similarly, Steer, Rissmiller, Ranieri and Beck (1993) found a two factor structure consisting of somatic and subjective symptoms involving 250 psychiatric inpatients. Additional factors have been identified in other studies 
that have used an exploratory approach to factor analysis. Leyfer, Ruberg and WoodruffBorden (2006), Osman, Kopper, Barriors, Osman and Wade (1997), Steer, Ranieri, Beck and Clark (1993), collectively identified four factors comprising similar dimensions of subjective, neurophysiological, panic and autonomic. The groups of study participants involved in the above studies included adults referred to an anxiety research ( $\mathrm{N}=194)$; undergraduate students $(\mathrm{N}=350)$; and outpatients with psychiatric disorders $(\mathrm{N}=470)$, respectively. The subjective dimension included items such as "unable to relax", "terrified" and "fear of worst happening". The neurophysiological dimension consisted of items such as "numbness", "hands trembling", and "dizzy". The panic dimension comprised of items such as "feelings of choking" and "difficulty breathing". The autonomic dimension consisted of items such as "feeling hot" and "sweating". Still, the highest number of dimensions identified was not limited to four factors. For example, using EFA, Borden, Peterson and Jackson (1991) reported a five-factor model in their study of 293 nonclinical undergraduate students. The identified dimensions included: subjective fear; somatic nervousness; neurophysiological; muscular/motoric; and respiration. Of the two-, four- and five-factor competing models reported in the literature, the model that has been tested most often is a four-factor model. The focus of this study was to examine the subjective dimension of anxiety, free from the associated physical symptoms. Therefore, factor analysis of the BAI concentrated on examining the latent structure of the scale using the data from current study participants and to demonstrate the confirmation of the unidimensional structure of the subjective subscale. The psychometric validity of the BAI established for the study population is 
reported later in the results section. The internal consistency reliability (Cronbach's alpha) of the BAI for this study was 0.89 .

\section{Depression.}

The Beck Depression Inventory (BDI) is a widely used self-report scale designed to measure the severity of depression (Beck, Steer, Ball \& Ranieri, 1996). The BDI is based on attitudes and symptoms observed in depressed individuals in clinical settings. The BDI, which consists of 21 items, can be divided into two subscales that assess different dimensions of depression: cognitive-affective domain and somatic-performance domain. Cognitive-affective domain (13 items) measures cognitively oriented symptoms, e.g., mood, suicidal thoughts, while somatic performance domain ( 8 items) measures non-cognitive symptoms, e.g., crying, loss of energy. The value of 0 to 3 is assigned to four choices in each item, and a total score is derived from a summation of individual scores. The range of scores is $0-63$ in which the higher scores represent a higher level of depression. The cut-off score for the BDI is dependent on the purpose of the research: screening or diagnostic. A score of $<10$ is assigned for a diagnosis of minimal depression; $10-18$ for mild to moderate depression; 19-29 for moderate to severe depression; and 30-63 for severe depression (Beck, Steer, \& Garbin, 1988). Furlanetto, Mendlowicz, and Bueno (2005) reported that a cut-off score of $9 / 10$ had $100 \%$ sensitivity and $83 \%$ specificity for detecting moderate and severe depression. Internal consistency of the BDI is reported in a meta-analysis as 0.86 for psychiatric patients and 0.81 for nonpsychiatric patients (Beck, Steer, \& Garbin, 1988). Concurrent validity was established by demonstrating a high correlation between the BDI and the Hamilton Psychiatric Rating Scale for Depression (HRSD). Mean correlation 
between the BDI and the HRSD was 0.73 for psychiatric patients, and 0.74 for nonpsychiatric patients. The BDI has been revised twice since the initial introduction (Beck, Ward, Mandelson, Mock, \& Erbaugh, 1961), first revised as the BDI-1A, and then to the current version as the BDI-II. In comparison to the old version, the BDI-II includes items that can measure severe depression. In addition, items for assessing body image, work difficulty, weight loss, and somatic concerns in the old version were replaced with items for assessing agitation, difficulty with concentration, and loss of energy (Beck, Steer \& Brown, 1996). The BDI is the most widely used self-report measure that has been employed in more than 2,000 empirical studies (Richter, Werner, Heerlein, Kraus, \& Sauer, 1998). In this study, the BDI-II was used to measure depression in people living with CHD. The BDI-II has been used as a screening tool for detecting depression in individuals in different health contexts, e.g., post stroke (Aben, Verhey, Lousberg, Lodder, \& Honig, 2002) and postpartum ( Beck \& Gable, 2001; Chaudron et al., 2010). Despite its wide use, the BDI-II has several potential psychometric issues.

The factor structure of the BDI-II has been examined in a number of studies involving different groups of participants: college students, medical outpatients, psychiatric outpatients, postpartum mothers, post-stroke patients, individuals suffering from depression/major depressive disorder, and those with chronic pain. The most frequently reported factor structures of the BDI-II consist of two- and three-factor models. The twofactor models that have been reported typically reflect the somatic and the cognitive dimensions of depression. However, a slight variation of the two-factor model exists because of the different pattern of salient items being loaded on each of the factors. The first 
variation of the model is labelled somatic-affective and cognitive (SA-C) and the second variation of the model is labelled cognitive-affective and somatic (CA-S) (Beck et al., 1996). In the SA-C model, SA (somatic-affective) factor encompasses salient loadings from items such as "tiredness or fatigue", "loss of energy", and "loss of pleasure", whereas the C (cognitive) factor obtains salient loadings from items such as "sadness", "self-dislike", and "suicidal thoughts" (Steer \& Clark, 1997). In the CA-S model, the pattern of factor composition is similar to the SA-C model except the salient symptoms reflecting the affective aspect is incorporated with the cognitive aspect of depression. Researchers have discovered that a few of the items pertaining to the affective dimension may shift from loading on one factor to another depending on the characteristics of the sample (Steer \& Clark, 1997).

Support for the SA-C model is found in a number of studies. For example, Steer, Ball, Ranieri and Beck (1999) used confirmatory factor analysis to determine the factor structure of the BDI-II in a sample of 210 outpatients diagnosed with DSM-IV depressive disorders. They found that second order depression composed of two first order factors representing the cognitive and noncognitive (affective and somatic symptoms) model had a good fit. Similar support was provided by Arnau, Meagher, Norris and Bramson (2001) in a study involving 333 primary care patients. Principal components analysis indicated that the SA-C model summarized their data parsimoniously. Viljoen, Iverson, Griffiths and Woodward (2003) provided additional evidence that supported the factor structure of the SA$\mathrm{C}$ model. They used exploratory factor analysis on the data from 127 individuals referred by 
their primary care physician and discovered that the SA-C model explained $53 \%$ of variance in their data.

The alternative factor structure of CA-S has also been supported by study findings. The CA-S factor structure aligns with findings involving studies of nonclinical college students. For instance, a group of 160 first-year university students completed the BDI-II questionnaire one week after their midterm examination (Steer \& Clark, 1997). Principal components analysis pointed to the two-factor model of the CA-S pattern as a parsimonious model to describe the data. Similarly, Beck, Steer and Brown's (1996) study involving a group of 120 students validated the two-factor structure of the CA-S in Dozois, Dobson and Ahnberg's (1998) study. Confirmatory factor analysis indicated that the CA-S model exhibited a good fit with the data obtained from 1,022 undergraduate psychology students. Whisman, Perez, and Ramel (2000) also used confirmatory factor analysis to analyze data from 576 students and were able to replicate the CA-S model - their model incorporated correlated errors for three pairs of items.

The three-factor model of the BDI-II is an alternative factor structure that has emerged in recent years. The three-factor model, also referred to as the general-factor model, consists of three first-order orthogonal factors - general (G) depression, somatic (S), and cognitive (C) (Ward, 2006). The G-S-C model specifies that all 21 items load on the G factor, and both the $\mathrm{S}$ and the $\mathrm{C}$ are residual factors. The items of the $\mathrm{S}$ factor are the same items as the items from Whisman et al.'s (2000) study; the items of C factor are the same items as the items from Beck et al.'s (1996) study. Both the S and the C factors are orthogonal to the $\mathrm{G}$ factor and to each other. Ward applied a confirmatory factor analysis to 
a combined set of data from previous psychometric investigation of the BDI-II (e. g., Beck et. al., 1996; Steer \& Clark, 1996) to evaluate the fit of the G-S-C model. Ward concluded that the three-factor G-S-C model had as good a fit, if not better, than the two-factor models reported in the literature. A different pattern of the three-factor model has been described by Harris and D'Eon (2008). They investigated a group of 481 patients suffering from chronic pain and found that the parsimonious model that fit the data consisted of a second-order construct (depression) with three first-order factors: negative attitude, performance difficulty, and somatic elements. The same three-factor model, albeit with a slightly different composition of items, was also described by Osman et al., (1997) in a study of 230 young adults.

The Cronbach's alpha of the BDI-II for this study was 0.96. Given the numerous factor structures reported for the BDI-II in the literature, the first step in the analysis involved establishing its psychometric validity for the study population.

\section{Coping.}

The examination of the way individuals cope with stressful events has been studied primarily from two different approaches - the style and the process approach. The style approach, analogous to the trait-oriented approach, considers coping as a property of the person, such as a personality disposition, with little emphasis placed on environmental influences. Within the context of the style approach, coping is represented as a trait that is stable and consistent over time and across various encounters. The process approach, on the other hand, considers coping as a process that changes over time and that is contextdependent (Folkman, Lazarus, Dunkel-Schetter, DeLongis \& Gruen, 1986; Lazarus, 1993). 
With the process approach, coping is studied as a response to the psychological and environmental demands of a specific stressor; coping is viewed as unstable and changes across different situations. The process approach asserts that individuals choose coping strategies based on their abilities and on the demands of a situation. In this study, the conceptualization of coping was consistent with the process approach and assumed coping to be a cognitive and behavioral response to the environmental demands of a specific situation.

The Ways of Coping Questionnaire (WCQ) is an instrument designed to assess coping as a process in accordance to the contextually oriented approach. The WCQ was developed based on the transactional theory of stress and coping by Lazarus (1961). The premise of the transactional theory is that the person and the environment are in a dynamic and mutually reciprocal relationship. Stress occurs when the person appraises a relationship between the person and the environment as taxing and his or her resources are insufficient for achieving well-being (Folkman, Lazarus, Gruen \& DeLongis, 1986). Folkman and Lazarus (1985) propose that coping enlists two primary functions, namely problem-focused and emotion-focused coping. Problem-focused coping deals with taking actions to change the stressful situation for the better, whereas emotion-focused coping deals with regulating emotional distress that arises from the stressful situation (Bouchard, Sabourin, Lussier, Wright \& Richer, 1997; Parker, Endler \& Bagby, 1993).

In this study, coping was assessed using the Ways of Coping Questionnaire (WCQ) (Folkman \& Lazarus, 1985). The WCQ is designed to assess a wide range of thoughts and behaviors that a person uses to deal with a specific stressor. Thus, a person is responding in relation to a particular stressful encounter. The WCQ, a revised form of the original Ways of 
Coping Checklist (WCC), consists of 66 items and responses are rated on a 4-point Likert scale that ranges from 0 (does not apply/or not used) to 3 (used a great deal). The instrument yields eight subscales: confrontative coping, distancing, self-controlling, seeking social support, accepting responsibility, escape-avoidance, planful problem-solving, and positive reappraisal. Of the eight subscales, two subscales (confrontive coping and planful problem-solving) measure problem-focused coping, while the other six subscales measure emotion-focused coping. The WCQ produces a score for individual subscales, however, it does not produce a total score. One of the weaknesses of the instrument, as acknowledged by the developers, is the inconsistency of the subscales produced in different studies. For example, the eight subscales developed in a study involving 150 participants who have experienced a wide range of stressors (Folkman, Lazarus, Dunkel-Schetter, DeLongis, \& Gruen, 1986) were not the same eight subscales produced in a study involving 108 undergraduate students coping with an exam (Folkman \& Lazarus, 1985). The authors recommended the use of eight subscales produced in a study involving 150 participants because the factor analysis used to derive the subscales was based on a broader sampling of subjects. (Folkman \& Lazarus, 1988). Despite the noted weakness, the WCQ was chosen for this study as it is consistent with the stress and coping theory - the theoretical basis of the instrument - that informs this study. Furthermore, the WCQ is the most widely used instrument for coping research (Tennen \& Herzberger, 1985). The WCQ has been used to study coping strategies in situations involving psychological loss, acute and chronic illness, postsurgery pain, loneliness, and personal failure (Felton \& Revenson, 1984; Parker, Endler, \& Bagby, 1993; Parkes, 1986). But most importantly, a lack of instruments with satisfactory 
psychometric properties to assess coping of individuals with CHD was the main reason for selecting the WCQ. The internal consistency reliabilities of eight subscales are reported to range from 0.61 to 0.79 . The score can be obtained in the form of either raw score or relative score in each scale. The raw score, the most common scoring form that reflects the coping effort, is produced from the sum of responses to items in each scale. Relative score reflects the contribution of each coping scale in relation to all of the scales combined. In other words, the relative score represents the proportion of effort indicated by each type of coping.

The WCQ has face validity because the individuals have reported using the strategies described in the questionnaire to deal with the stressor. Construct validity is claimed to have been established from the congruence of study findings and predictions from the stress and coping theory (i.e., coping consists of problem-focused and emotion-focused strategies). Internal consistency reliabilities of the three subscales of the WCQ for this study were as follows: problem-solving 0.94, wishful-thinking 0.91, and denial/avoidance 0.93 .

\section{Social support.}

Stewart (1989) describes that social support is generally studied in three dimensions: social network structure (sources, received vs. perceived, duration), function (emotional, instrumental, appraisal, informational), and nature (positive vs. negative). Thus, the core dimensions of social support assessed pertain to existence or quantity (structure), content (function) and quality (nature). Social support consists of multi-dimensions and thus, a single instrument would be insufficient to capture the comprehensive picture of the construct. Although using multiple measures to capture the different aspects of social support is desirable, the constraints associated with conducting research make this goal impractical to 
achieve. In the current study, the use of multiple measures of social support would have made the analysis of the structural equation modeling complex and problematic. When the researcher is limited to a use of a single measure of support, Cohen, Underwood and Gottlieb (2000) advocate for a measure that assesses both perceived and received supports. The Berlin Social Support Scales was an appropriate measure for this purpose as it not only measures both perceived- and received social supports, it also assesses the negative aspect of received social support. The Berlin Social Support Scales (BSSS, Schwarzer \& Schulz, 2000) is a 52-item instrument that encompasses six subscales: perceived social support, need for support, support seeking, received support, provided support, and protective buffering scale. The BSSS was used to measure cognitive and behavioral dimensions of social support and to examine quantity, type and function of social support. The response to each item is rated on a 4-point Likert-type scale that ranges from strongly disagree (1) to strongly agree (4). The score can be generated in two ways: a total score by adding up responses of all items, or generating a mean score from each subscale. For the purpose of this study, only the two subscales, perceived actual social support ( 8 items) and received available social support (11 items), were used. Both perceived and received social support are able to assess different function of support - emotional and instrumental support - and separate scores for each function of support can be produced.

For received support, the additional dimension of satisfaction with given support from "a person closest to" the participant was also assessed. Furthermore, received social support included items that assess negative aspects of support, e.g., "this person (a person closest to the participant) did not show much empathy for my situation", "this person 
complained about me". Internal consistency reliability of perceived social support and received social support is 0.83 and 0.83 , respectively. The BSSS was originally designed to study the coping behavior of people experiencing cancer surgery (Schulz \& Schwarzer, 2004). The BSSS has been used in the studies involving cardiac patients (Schroder, Schwarzer, \& Endler, 1997; Schroder, Schwarzer, \& Konertz, 1998), cancer patients and their spouses (Schulz \& Schwarzer, 2004), and recipients of tumor surgery (Boehmer, Luszczynska, \& Schwarzer, 2007). The Cronbach's alpha was 0.84 for perceived social support and 0.96 for received social support.

\section{Cardiac symptoms.}

The Cardiac Symptom List (CSL) is a measure developed to assess the types and frequency of discomfort experienced by patients with ischemic heart disease. The CSL consists of six items that are rated on a 4-point Likert-like scale that ranges from "never" (0) to "very often" (3). Six items represent the six symptoms: fatigue, arm or neck pain, chest pressure, breathlessness, heart pounding or racing, and chest pain. The content validity was established with a group of nurses with expertise in coronary care (Frunchak, 1989). The alpha coefficient for symptom frequency was 0.76 and for number of symptoms was 0.63 (Frunchak, 1989). Purden (1995) reported that the scale is likely measuring unidimension of cardiac symptoms. The CSL was used in this study as one of the indicators of physical functioning. 


\section{Physical functioning.}

The Short-Form Health Survey -36 (SF-36) v2 is an instrument designed to measure the eight domains of health status of individuals experiencing a disease or illness (Ware et al., 2007). The eight health domains include: physical functioning, role participation with physical health problems, bodily pain, general health, vitality, social functioning, role participation with emotional health problems, and mental health. Of the eight domains of the scale, the subscale of physical functioning was used to measure the functional status of the study participants of this study. The physical functioning subscale consists of 10 items and the response is rated on a 3-point Likert-type scale that ranges from "yes, limited a lot" (1) to "no, not limited at all" (3). The total raw score is computed by adding up the responses of all items. The total raw score can be transformed to a 0-100 scale score using the formula: [(total raw score - 10)/20] x 100. The SF-36v2 has been used extensively in studies involving various illnesses, e.g., cardiovascular disease, cancer, chronic obstructive pulmonary disease, depression, rheumatoid arthritis, sleep disorders, stroke, and diabetes. The reported reliabilities (Cronbach's alpha) of the physical functioning subscale range from 0.91 to 0.94 (Ware et al, 2007). The SF-36v2 has demonstrated evidence of construct validity (via principal components analysis), as well as content, concurrent, and criterion validities (Ware et al, 2007).

\section{Ethical Considerations}

The researcher obtained the approval of the University of British Columbia Behavioural Research Ethics Board, and the St. Paul's Hospital Research Ethics Board 
(Preface). Participants of the study were given rights to privacy, informed consent (Appendix A), option for voluntary withdrawal, and protection from harm. Confidentiality of data was maintained and any identification information was accessible only to the investigator.

\section{Data Analysis}

\section{Data screening.}

The data were entered into a data file using the software SPSS version 19. Prior to the start of the analysis, the data were screened for accuracy of data entry, coding errors, multiple responses, missing values, and outliers. When, in error, the participant provided more than one response to the Likert scale items, the response entered was randomly selected. Frequency and cross tabulation tables were used to check for missing values and outliers. In the cases of outliers, the data were examined to ensure that the data entry was valid and that the values were within the possible range of the variables (Tabachnick \& Fidell, 2007). Questionnaires that were missing a significant amount of data (> 10\%) on major study variables were excluded from the raw data file $(\mathrm{N}=7)$. Examination of data showed that all study variables had less than $5 \%$ of missing data, which did not present a significant problem for the analysis (Tabachnick \& Fidell, 2007). A more detailed discussion on the management of missing data is presented later in the result section.

\section{Scale of variables.}

The data for this study were collected using a set of measures consisting of three to four ordered categories. Joreskog and Sorbom (1993) remind us that ordinal and continuous 
variables differ significantly in the way they operate and must not be treated equivalently in analyses. With ordinal variables, observations reflect responses to a set of ordered categories, whereas with continuous variables, responses reflect responses on a continuous scale in which the response could take any score within the range of possible values (Byrne, 1998). Ordinal variables are assumed to have an underlying continuous variable with each pair of thresholds reflecting a portion of the continuous scale. The continuous scale of the construct is divided into a fixed number of ordered categories (DiStefano, 2002). The analyses of ordinal variables are based on a polychoric (correlation between both ordinal variables) or a polyserial (correlation between an ordinal variable and a continuous variable) correlation matrix. The analysis with categorical data operates under three assumptions: 1) each categorical variable has an underlying counterpart latent variable, which is continuous and normally distributed, 2) sample size is sufficiently large to reliably estimate the correlation matrix, and 3) has minimum number of observed variables (Byrne, 2012).

The distributions of the observed variables in this study were non-normal. Since the data obtained from Likert scaling are considered ordinal data (Ware \& Benson, 1975), the variables in this study were treated as ordinal/categorical variables and the analyses relied on a statistical technique (i.e., mean- and variance-adjusted weighted least square) that accommodates the conditions described above (i.e., range of sample size from 100 to 1,000, non-normal distribution of data) (Brown, 2006).

\section{Model specification.}

A model represents the hypothesized relationships between the latent variables and their indicators (observed variables), and the relationships among the latent variables 
(between endogenous and exogenous variables) (Byrne, 2012). A two-step approach was used in the model testing.

\section{Step one: Testing of measurement models.}

The measurement models of the instruments used in the study were established using either an exploratory factory analysis (EFA) or confirmatory factor analysis (CFA). EFA is designed to explore the number of underlying factor structure of a set of observed variables and to determine how, and to what extent, the observed variables are linked to latent variables (Byrne, 2012). CFA, on the other hand, is used to confirm the relationship between a set of observed variables and the underlying factors that was hypothesized a priori by the researcher (Byrne, 2012). When the factor structure of the measure was not well established, an EFA was performed to determine the number of latent constructs and the underlying factor structure of the instrument. For example, validating the factor structure of the WCQ was challenging and an EFA had to be performed to ascertain the underlying factor structure. Since the data of this study consist of categorical/ordinal variables that were nonnormally distributed, the appropriate estimator was the mean- and variance-adjusted weighted least square (WLSMV) available in the Mplus (Muthen \& Muthen, 2010). The geomin oblique rotation was used for the EFAs. Cases with missing values were excluded with the pairwise deletion. The following criteria were used to select items to be included in the factor structure of the WCQ: 1) factor loading $\geq .4$ (Brown, 2006; Nunnally, 1978), 2) items selected for given factors must correspond to theoretical dimensions specified by the Transactional Theory of Stress, and 3) items selected must yield meaningful contribution and enhance interpretability of the corresponding structure. For the purpose of the analysis, some 
crossloading of items was permitted. Crossloading was allowed as the purpose of the EFA was to compute a subscale that will then be used for the structural equation modeling (SEM) analysis. SEM is a confirmatory modeling technique use to ascertain the validity of a model (Byrne, 2012). Retaining the item despite the condition of crossloading preserved its contribution to a corresponding factor and enhanced the ability to measure the latent factor.

CFA was used when validating the factor structure of well-established instruments. The validation of the measures was established by testing the hypothesized relationships between the latent constructs and their observed variables. The specification of CFA models was guided by the theoretical knowledge of the constructs and previous empirical research. Factor loadings and item thresholds of the model were estimated using the WLSMV. The metric of the latent factors were achieved by setting the first indicator of each factor to 1.0 (Brown, 2006). In multidimensional models (i.e., WCQ), latent factors were allowed to correlate with one another. When a selected number of subscales were extrapolated from an instrument (i.e., BSSS), the fit of the measurement of the subscales used in the SEM was tested to ensure at least an acceptable fit.

\section{Step two: Testing of the structural models.}

Following the factor analyses to establish the validity of measurement models, the structural models were specified and tested (Schumaker \& Lomax, 2004). Based on the conceptual model of the study (Figure 1), the overall structural models of the relationships tested were: the effects of types of social support and types of coping strategies on psychological distress. More specifically, perceived and received social supports were hypothesized to influence psychological distress either directly or indirectly through different 
types of coping strategies. Two separate structural models were tested: one with anxiety as an endogenous variable and one with depression as an endogenous variable. The mediation effects of the variable were tested by evaluating the statistical significance of the indirect effect (McKinnon, 2008).

\section{Estimation method.}

For many years, some of the most widely used estimation methods have been the maximum likelihood (ML) and robust ML (MLM). The ML is not an ideal choice of estimator as it is sensitive to the number of ordered response categories as well as nonnormal distribution (Finny \& DeStefano, 2006). The variables of this study are ordinal/categorical and thus, should not be treated as continuous variables (Brown, 2006). When ordinal/categorical variables are analyzed as continuous variables, the following consequences may occur: 1) inflated correlation coefficients, 2) inflated $\chi^{2}$ values, 3) underestimation of factor loadings, factor correlations and standard errors, and 4) incorrect estimation of residual variances (Byrne, 2012). For data involving categorical/ordinal variables, the estimation method of least squares is recommended (Finny \& DeStefano, 2006). Three primary estimation methods for ordinal variables are available: unweighted least squares (ULS), weighted least squares (WLS), and diagonally weighted least squares (DWLS). Mean- and variance-adjusted weighted least square (WLSMV) is an estimator available for the Mplus program that uses a diagonally weighted matrix (W), robust standard errors, and a robust mean- and variance-adjusted $\chi^{2}$ statistic (Muthen \& Muthen, 2010). 
According to Brown (2006), the WLSMV estimator is the best method for the modeling of categorical data, and for this reason it was selected as the estimator for analyses.

\section{Assessment of fit.}

The purpose of assessing the fit of the model is to determine whether the hypothesized model possesses the ability to reproduce the data (i.e., variance-covariance matrix). One of the prominent fit indices that has long been used for testing the model fit is the likelihood ratio $\chi^{2}$ statistic. However, $\chi^{2}$ statistic has a well-known weakness due to its

sensitivity to sample size of $>400$ (Byrne, 2012). Another weakness of the $\chi^{2}$ statistic is that it operates under the premise that the sample covariance matrix (S) is equal to the restricted covariance matrix $(\Sigma)$. Recently, a group of alternate fit indices has been introduced that divides into two categories: incremental or comparative and absolute. Of the two types of fit indices, the incremental indices are most widely used (Hu \& Bentler, 1999). The incremental fit indices assess the improvement in fit of a hypothesized model in comparison to a baseline model (Hu \& Bentler, 1999). The absolute fit indices measure the extent to which the sample data can be reproduced by an a priori model. For absolute fit indices, the degree of improvement is not based on any reference model. Two of the most commonly used incremental fit indices, also available in Mplus software, are the comparative fit index (CFI) and Tucker-Lewis Fit Index (TLI) (Byrne, 2012). The CFI measures the fit between the hypothesized model and the less restricted baseline model. The CFI is a normed index, meaning, its value can range from zero to 1.0 , with values closer to 1.0 reflecting a wellfitting model. The cut-off value close to .95 represents a good-fitting model. The TLI is a 
non-normed index, meaning its values can reach above 1.0. The cut-off value of $\geq .95$ suggests a good fit (Hu \& Bentler, 1999). One of the absolute fit indices used in the study was the root mean square of approximation (RMSEA) that measures the degree to which the hypothesized model fits the sample data. In contrast to the incremental fit indices whose value increases as goodness-of-fit increases, the value of fit indices decreases as the goodness-of-fit increases. Absolute fit indices consider the best fitting model as a fit of zero. The value of $<.05$ indicates good fit, and $\leq .08$ indicates a reasonable fit (Browne \& Cudeck, 1993). Thus, for the purpose of testing the fit of the model in this study, the following criteria, $\mathrm{CFI} \geq .95$ and $\mathrm{RMSEA} \leq .08$, were used as being reflective of an adequate-fit model (Brown, 2006).

Additional means to assess the model fit included the examination of the: 1) residual correlation matrix, 2) modification indices (MI), and 3) factor loadings. The residual correlation matrix indicates the difference between the observed and implied matrices (significant $=\geq|0.10|$ ) and points to areas of mis-specification in the model. The MI reflects the improvement in fit that would result if an additional path was added to the model (default criteria in the Mplus $\geq 10.0$ ). The factor loading $\geq .40$, unstandardized parameter estimate/standard error $\geq|1.96|$ was used as reference values for further assessing the model (Brown, 2006; Schumacker \& Lomax, 2004). 


\section{Chapter 4}

\section{Results}

The following chapter presents the results of the study in three parts. The first part describes the characteristics of the sample including the sociodemographic and clinical characteristics. The second part examines the psychometric properties of the instruments used in the study. The third part presents the results of model testing and addresses the research questions.

\section{Characteristics of the Sample}

The sociodemographic characteristics of the sample are summarized in Table 1.

The gender of participants was nearly evenly represented between male (46.7\%) and female (53.3\%). The mean age of males was 35.7 years and their age ranged between $18^{*}$ to 87 years, while the mean age of females was 37.8 years with the age range between 17 to 77 years. More than half of the participants $(n=164,60.2 \%)$ were in the age group of 20 's and 30's. The majority of the participants were either married or in common-law relationships $(n=145,53.3 \%)$ or single $(n=112,41.2 \%)$. More than half of the participants had completed at least part of a college program, and $19 \%$ of these individuals had a post-graduate degree. Half of the participants $(n=134,49.3 \%)$ were employed in a full time basis, while

* For two of the participants who did not meet the age criteria and yet expressed an interest to participate in the study, their respective parents provided the consent. 
Table 1: Sociodemographic Characteristics of the Participants $(\underline{n}=272)$

\begin{tabular}{|c|c|c|c|c|}
\hline \multirow[t]{2}{*}{ Characteristics } & \multirow[t]{2}{*}{ Sample (\%) } & \multirow[b]{2}{*}{$\underline{\mathrm{M}}$} & \multirow[b]{2}{*}{$\underline{\mathrm{SD}}$} & \multirow[b]{2}{*}{$\underline{\text { RANGE }}$} \\
\hline & & & & \\
\hline \multicolumn{5}{|l|}{ Sex } \\
\hline Male & $127(46.7)$ & & & \\
\hline Female & $145(53.3)$ & & & \\
\hline \multicolumn{5}{|l|}{ Age $(\%)^{(\mathrm{n}=272)}$} \\
\hline Male & & 35.7 & 14.7 & $18-87 *$ \\
\hline Female & & 37.8 & 14.8 & $17-77 *$ \\
\hline$<20$ & $19(7.0)$ & & & \\
\hline $20-29$ & $93(34.2)$ & & & \\
\hline 30-39 & $71(26.1)$ & & & \\
\hline $40-49$ & 31 & & & \\
\hline $50-59$ & $29(10.7)$ & & & \\
\hline $60-69$ & $22(8.1)$ & & & \\
\hline$>70$ & $7(2.6)$ & & & \\
\hline \multicolumn{5}{|l|}{ Marital Status $^{(\mathrm{n}=272)}$} \\
\hline Single & $112(41.2)$ & & & \\
\hline Married/common-law & 145 & & & \\
\hline Divorced/separated & $15(5.5)$ & & & \\
\hline \multicolumn{5}{|l|}{ Education $^{(\mathrm{n}=270)}$} \\
\hline High school or less & 37 (13.7) & & & \\
\hline $\begin{array}{l}\text { Trade/technical/vocational } \\
\text { training }\end{array}$ & $47(17.4)$ & & & \\
\hline Some college or higher & $186(68.9)$ & & & \\
\hline \multicolumn{5}{|l|}{ Income $^{(\mathrm{n}=262)}$} \\
\hline$<20,000$ & $84(32.1)$ & & & \\
\hline $21,000-40,000$ & $70(26.7)$ & & & \\
\hline $41,000-60,000$ & $44(16.8)$ & & & \\
\hline$>61,000$ & $64(24.4)$ & & & \\
\hline
\end{tabular}

(continued) 
Table 1: Sociodemographic Characteristics of the Participants $(\underline{n}=272)$ (continued)

\begin{tabular}{|c|c|}
\hline Characteristics & Sample (\%) \\
\hline \multicolumn{2}{|l|}{ Occupation $^{(\mathrm{n}=268)}$} \\
\hline Professional/specialized technology & $11(4.1)$ \\
\hline Health care & $22(8.2)$ \\
\hline Education/training & $22(8.2)$ \\
\hline Business/management & $33(12.3)$ \\
\hline Skilled labour & $57(21.3)$ \\
\hline Unskilled & $69(25.7)$ \\
\hline Student & $22(8.2)$ \\
\hline Not working & $32(11.9)$ \\
\hline \multicolumn{2}{|l|}{ Employment ${ }^{(\mathrm{n}=270)}$} \\
\hline Full-time & $134(49.6)$ \\
\hline Part-time & $32(11.9)$ \\
\hline Casual/contract & $24(8.9)$ \\
\hline Student & $39(14.4)$ \\
\hline Unemployed & $41(15.2)$ \\
\hline \multicolumn{2}{|l|}{ Past diagnosis } \\
\hline Anxiety ${ }^{(\mathrm{n}=266)}$ & $53(19.9)$ \\
\hline Depression $^{(\mathrm{n}=259)}$ & $64(24.7)$ \\
\hline \multicolumn{2}{|l|}{ Current experience ${ }^{(\mathrm{n}=258)}$} \\
\hline Anxiety or depression & $53(22.5)$ \\
\hline \multicolumn{2}{|l|}{ Self-evaluation of current health ${ }^{(\mathrm{n}=265)}$} \\
\hline Excellent & $33(12.5)$ \\
\hline Very good & $102(38.5)$ \\
\hline Good & $91(34.3)$ \\
\hline Fair & $31(11.7)$ \\
\hline Poor & $8(3.0)$ \\
\hline \multicolumn{2}{|l|}{$\begin{array}{l}\text { Health compared to } \\
1 \mathrm{yr} \mathrm{ago}^{(\mathrm{n}=272)}\end{array}$} \\
\hline Much better & $35(12.9)$ \\
\hline Somewhat better & $51(18.8)$ \\
\hline About the same & $136(50.0)$ \\
\hline Somewhat worse & $39(14.3)$ \\
\hline Much worse & $11(4.0)$ \\
\hline
\end{tabular}

* For two participants who were less than 19 years of age, their parents provided the consent to participate in the study. 
approximately $15 \%(\mathrm{n}=15.2)$ of the participants were without employment. A similar proportion of the participants were in skilled (21\%) and unskilled labour (25.4\%), with the remaining participants employed in various occupations including professional/specialized/technological (4\%), health care (8.2\%), education/training $(8.2 \%)$, and student (11.8\%). The mean annual income of participants was fairly evenly distributed with $32 \%$ earning less than $\$ 20,000 ; 26.7 \%$ earning between $\$ 21,000-40,000 ; 16.8 \%$ earning $\$ 41,000-60,000$; and $24.4 \%$ earning more than $\$ 61,000$.

The anxiety level of study participants varied from minimal to severe, based on the criteria established by Beck et al. (1988). The score of BAI can range from 0 to 63, of which 0-7 represents the "minimal" level, 8-15 the "mild" level, 16-25 the "moderate" level, and 26-63 the "severe" level of anxiety. Half (50.6\%) of the study participants reported a minimal level of anxiety, $25.5 \%$ reported a mild level, $15.3 \%$ reported a moderate level, and $3.7 \%$ reported a severe level of anxiety.

The scores of depression also varied across four different levels based on the criteria developed by Beck et al. The score of BDI-II can range from 0 to 63, of which 0-9 represents the minimal level, 10-18 the mild level, 19-29 the moderate level, and 30-63 the severe level of depression. The pattern of the participants' depression level was a little more skewed in comparison to their pattern of anxiety level. More than three quarters (74.3\%) of the participants reported a minimal level, $12.5 \%$ a mild level, $7.0 \%$ a moderate level, and $3.7 \%$ a severe level of depression.

The participants' severity of congenital heart disease was not easily determined using the existing classification of the CHD. The nomenclature and classification are largely 
based on embryologies which are complex and confusing to many health care personnel. A more recent classification was developed by the American College of Cardiology Task Force 1 of the $32^{\text {nd }}$ Bethesda Conference and consists of three categories: simple lesions, moderate lesions, and severe lesions (Taussig, 2011). However, the ACHD program at the study hospital did not use any of the classification of CHD severity, and as a result, the information was not available. Thus, a decision was made to assess the physical and functional status of the participants using the Cardiac Symptom List (Purden, 1995) and the physical subscale of SF-36. The Cardiac Symptom List (CSL) assesses the types and frequency of cardiac symptoms commonly associated with cardiac disease:1) tires easily, 2) arm or neck pain, 3) heaviness, burning or pressure in chest, 4) breathlessness, 5) heart pounding or racing, and 6) chest pain. Almost half $(\mathrm{n}=123,45 \%)$ the participants admitted to being easily tired. A number of participants experienced various cardiac symptoms at the frequency of either fairly often or very often: arm or neck pain $(n=69,25.4 \%)$, heaviness, burning or pressure in chest $(n=39,14.4 \%)$, breathlessness $(n=36,13.3 \%)$, heart pounding or racing $(n=49,18 \%)$, and chest pain $(\mathrm{n}=31,11.4 \%)$. Between $11-25 \%$ of the participants reported frequently experiencing some cardiac symptoms (Figure 4). In terms of the functional level, the proportion of participants who were limited in performing various activities to at least "a little" degree were $67.6 \%(n=184)$ with the vigorous activities (i.e., running, lifting heavy objects), $24.3 \%$ ( $\mathrm{n}=66$ ) with the moderate activities (i.e., moving a table, pushing vacuum), $18.3 \%(\mathrm{n}=50)$ with climbing one flight of stairs, $12.8 \%(\mathrm{n}=35)$ with walking one hundred yards, and 5.7\% ( $\mathrm{n}=16)$ with bathing or dressing (Figure 5). The mean score of the Short Form Survey 36 v2 (SF-36v2) for the study participants ( $\mathrm{n}=269)$ was 82.22 (SD 22.55). The 
Figure 4. Types and Frequency of Common Cardiac Symptoms Experienced by the Sample (Measured With the Cardiac Symptom List)
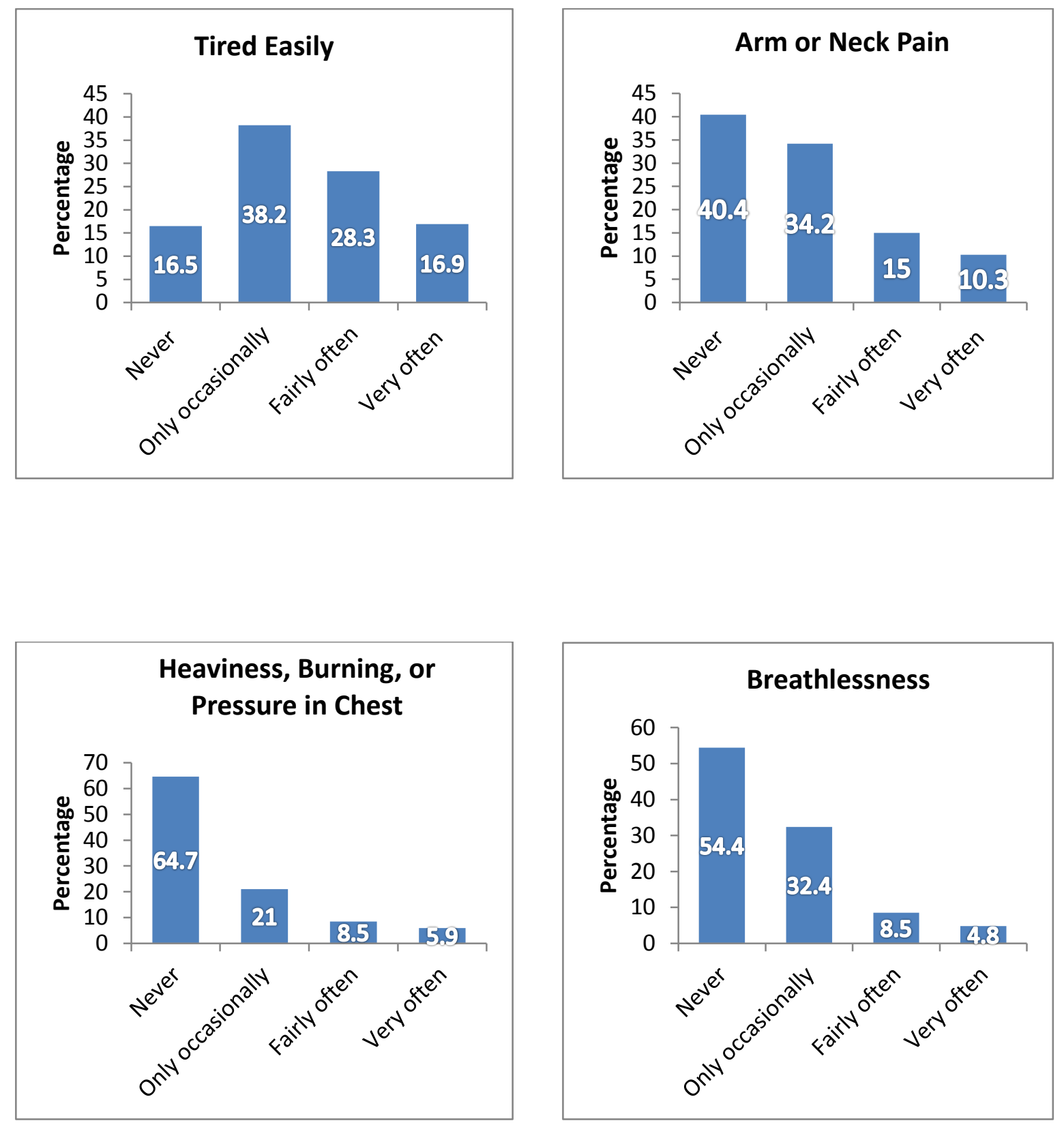

(Continued) 
Figure 4. Types and Frequency of Common Cardiac Symptoms Experienced by the Sample (Measured With the Cardiac Symptom List) (continued)
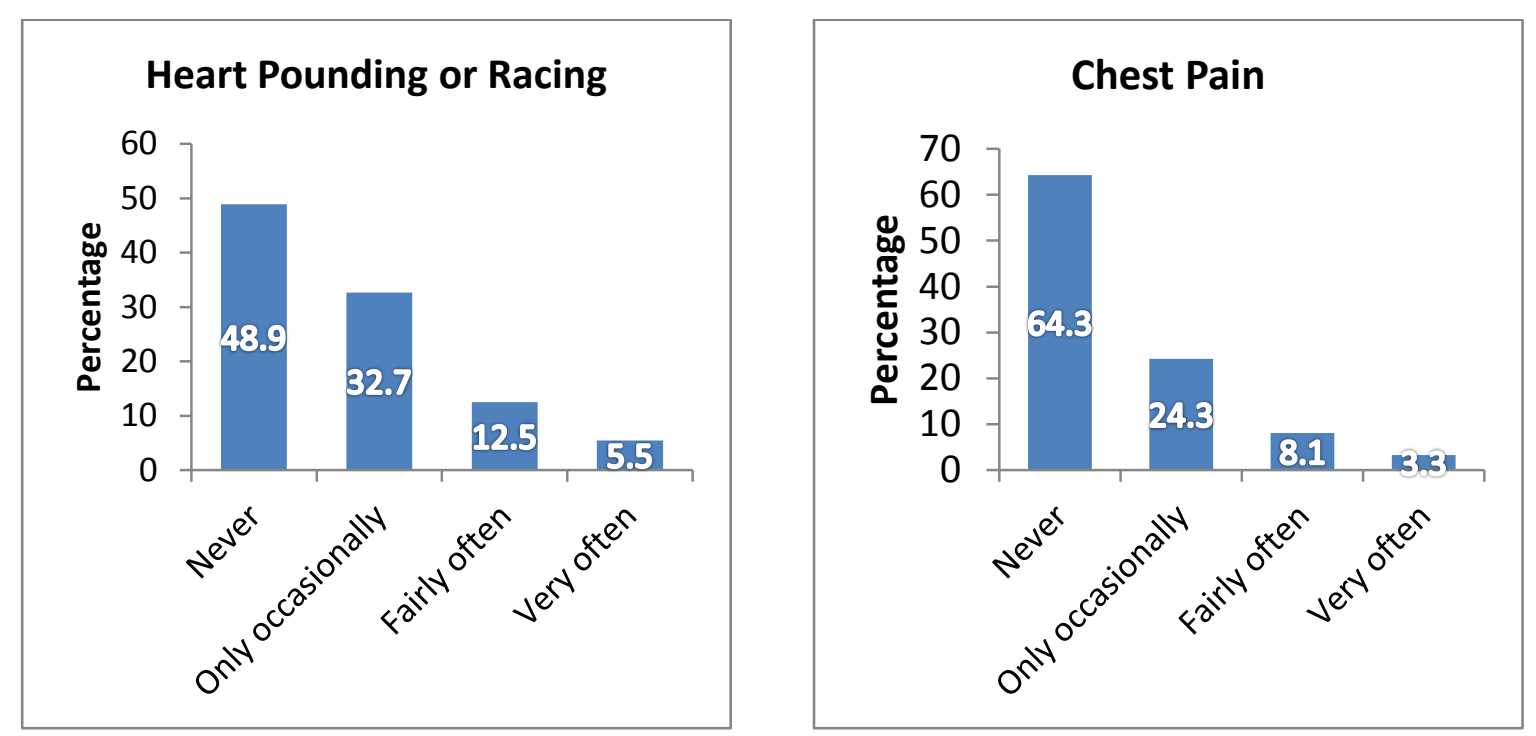
Figure 5. Short Form (SF-36)v2 Health Survey (Physical Functioning Subscale)
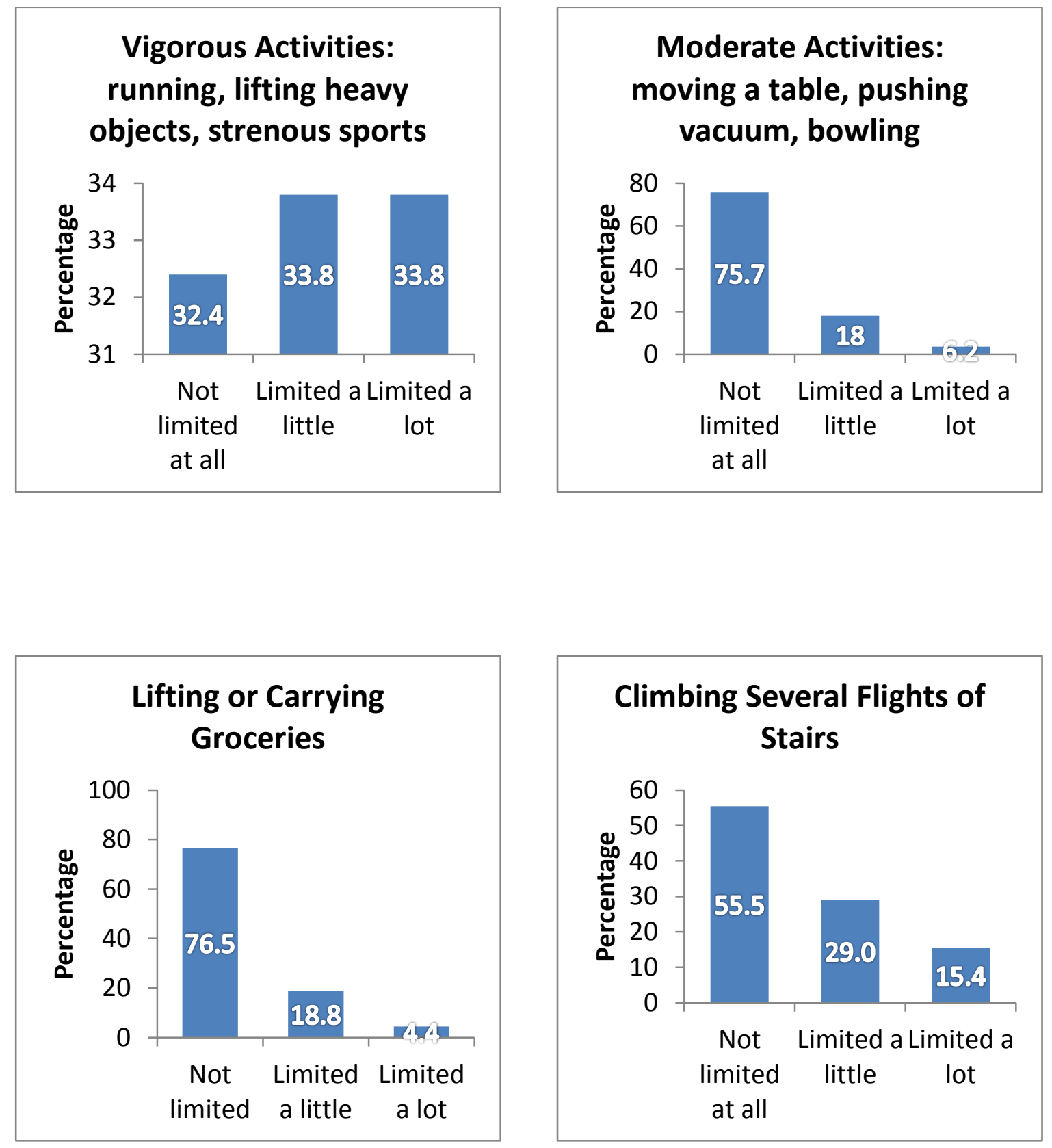

(Continued) 
Figure 5. Short Form (SF-36)v2 Health Survey (Physical Functioning Subscale) (continued)
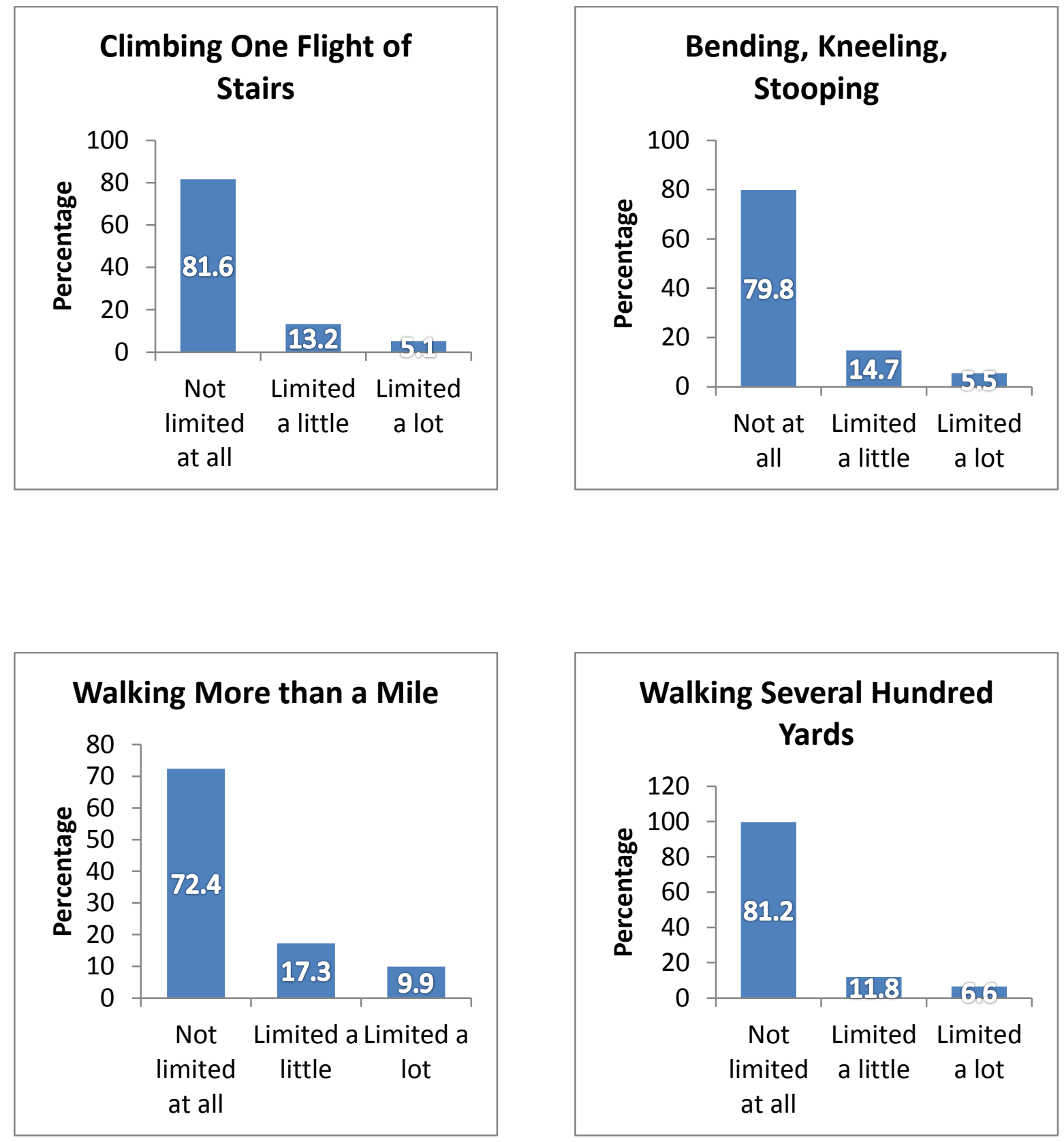

(Continued) 
Figure 5. Short Form (SF-36)v2 Health Survey (Physical Functioning Subscale) (continued)
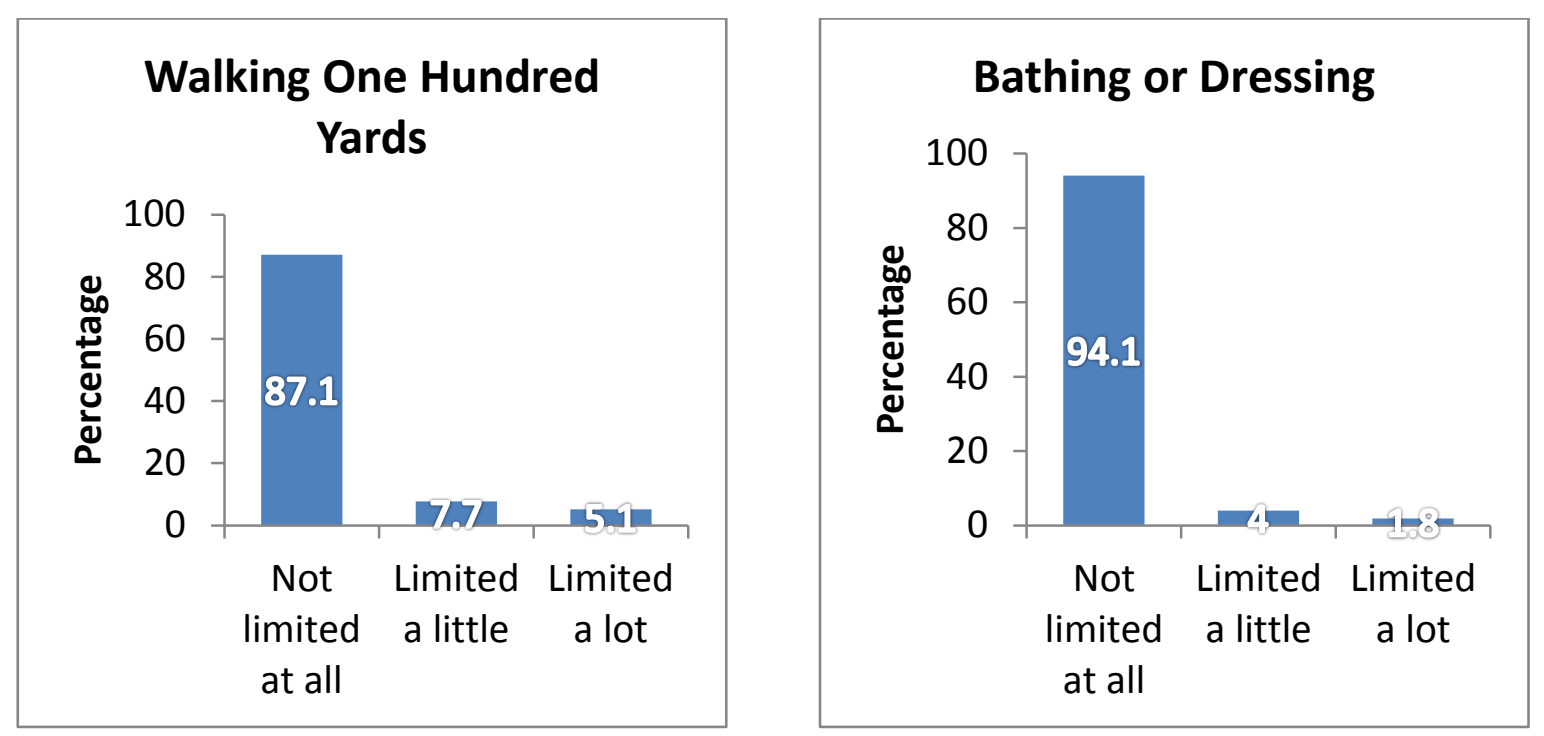
mean score of the SF-36v2 in 1998 U. S. general population is 83.29 (SD 23.76) (Ware et al., 2007). Therefore, the functional level of study participants was slightly lower in comparison to the normal population. Despite experiencing various cardiac symptoms and limited functional levels, more than three quarters $(n=231,84.9 \%)$ of the participants rated their health to be in a "good" or "excellent" state, and a similar proportion of (81.7\%) of participants thought that their health was at least same or better in comparison to one year ago. A small proportion of participants $(n=8,2.9 \%)$ perceived their health as poor. In terms of the past history of psychological disorder, 53 people $(19.9 \%)$ had been diagnosed with anxiety, of which 37 (69.8\%) were female. Having a history of anxiety was significantly lower in males than females $\left(\chi^{2}=7.5, \mathrm{df}=1, \mathrm{p}<0.01\right)$.

In terms of depression, 64 participants had been diagnosed with the condition, of which 41 were female. Similar to the history of anxiety, the history of depression was also significantly lower in males than females $\left(\chi^{2}=4.5, \mathrm{df}=1, \mathrm{p}=0.03\right)$. Of the individuals with history of psychological distress (i.e., anxiety, depression), 53 individuals (male=21, female =32) were still experiencing the distress at the time of data collection.

In summary, the participants of this study consisted of relatively young individuals whose gender was evenly represented. They were a fairly well educated group in which the majority of the members perceived themselves to be in good health. Although up to $25 \%$ of participants were experiencing some degree of cardiac symptoms and up to $68 \%$ were experiencing at least some degree of physical limitation, many individuals reported their health to be good and considered their health to be at least the same or better as the year prior. 


\section{Step 1: Testing of Measurement Models}

\section{Psychometric properties of measures.}

Factor analyses - EFA and CFA -were used to examine the nature of relationships among latent variables and observed variables, and to establish the measurement models. Structural equation modeling was then used to specify the hypothesized structural model (Schumacker \& Lomax, 2004). All analyses were performed using the Mplus version 6.0 software program.

\section{Beck Anxiety Inventory.}

The establishment of the factor structure of the Beck Anxiety Inventory (BAI) needed to draw on a combination of factor structures described in previous studies as despite the use of the measure in different studies, the factor structure was inconsistent (Table 2). The factor structure of the BAI has varied with number of factors ranging from two to five. A limited number of analyses using confirmatory factor analysis have been conducted to validate the factor structure of the BAI. Between the two-factor and the four-factor competing models, only the four factor model has been successfully replicated. The goodness-of-fit for the evaluation of the two-factor model has often reflected a poor fit. Consistent with the trend reported in the literature, Osman, Barrios, Aukes, Osman and Markway (1993) were unsuccessful in validating Beck's (1988) two-factor model. However, they were able to successfully replicate the four-factor model in their study involving 350 undergraduate students. Findings of their study supported both the four-factor oblique model 


\section{Table 2: Items of the Beck Anxiety Inventory}

\begin{tabular}{|c|c|}
\hline Item & Description \\
\hline 1 & Numbness or tingling \\
\hline 2 & Feeling hot \\
\hline 3 & Wobbliness in legs \\
\hline 4 & Unable to relax \\
\hline 5 & Fear of worst happening \\
\hline 6 & Dizzy or lightheaded \\
\hline 7 & Heart pounding/racing \\
\hline 8 & Unsteady \\
\hline 9 & Terrified or afraid \\
\hline 10 & Nervous \\
\hline 11 & Feeling of choking \\
\hline 12 & Hands trembling \\
\hline 13 & Shaky/unsteady \\
\hline 14 & Fear of losing control \\
\hline 15 & Difficulty in breathing \\
\hline 16 & Fear of dying \\
\hline 17 & Scared \\
\hline 18 & Indigestion \\
\hline 19 & Faint/lightheaded \\
\hline 20 & Face flushed \\
\hline 21 & Hot/cold sweats \\
\hline
\end{tabular}


$\left(\chi 2(\mathrm{df} 179)=656.44, \mathrm{NFI}^{*}=0.91, \mathrm{NNFI}^{+}=0.92, \mathrm{CFI}=0.93\right)$, and the second-order four factor model $(\chi 2($ df 180 $)=656.73, \mathrm{NFI}=0.91, \mathrm{NNFI}=0.92, \mathrm{CFI}=0.93)$.

The data of this study were evaluated for the fit of the one-, two- and four-factor models (Table 3). Borden et al.'s five-factor model was considered a poor model for an evaluation as it contained five items that each cross-loaded to more than one factor. Therefore, the fit of the five-factor model was not tested. Consistent with the findings of Osman et al., the fit of all models were poor. Of all the factor models tested, Osman et al.'s model seemed most promising with slight modification. When their second order model was changed to the first order four-factor model, the fit of the model became an acceptable level (Table 4). Although the four-factor model demonstrated an acceptable fit with the data, the focus of this study was the subjective aspect of anxiety free from physical symptoms, the decision was made to retain the subscale subjective dimension of anxiety. Therefore, only the subscale of the subjective factor was used to reflect the anxiety as one of the outcome latent variables. The subjective factor included the items 4 (unable to relax), 5 (fear of worst happening), 9 (terrified or afraid), 10 (nervous), 14 (fear of losing control), 16 (fear of dying), and 17 (scared) of the BAI (Figure 6). The goodness-of-fit indices of the subjective factor were: RMSEA 0.067 and CFI 0.994.

\footnotetext{
${ }^{*} \mathrm{NFI}=$ normed fit index; ${ }^{+} \mathrm{NNFI}=$ non-normed fit index
} 
Table 3: $\quad$ Factor Models of BAI Described in the Literature

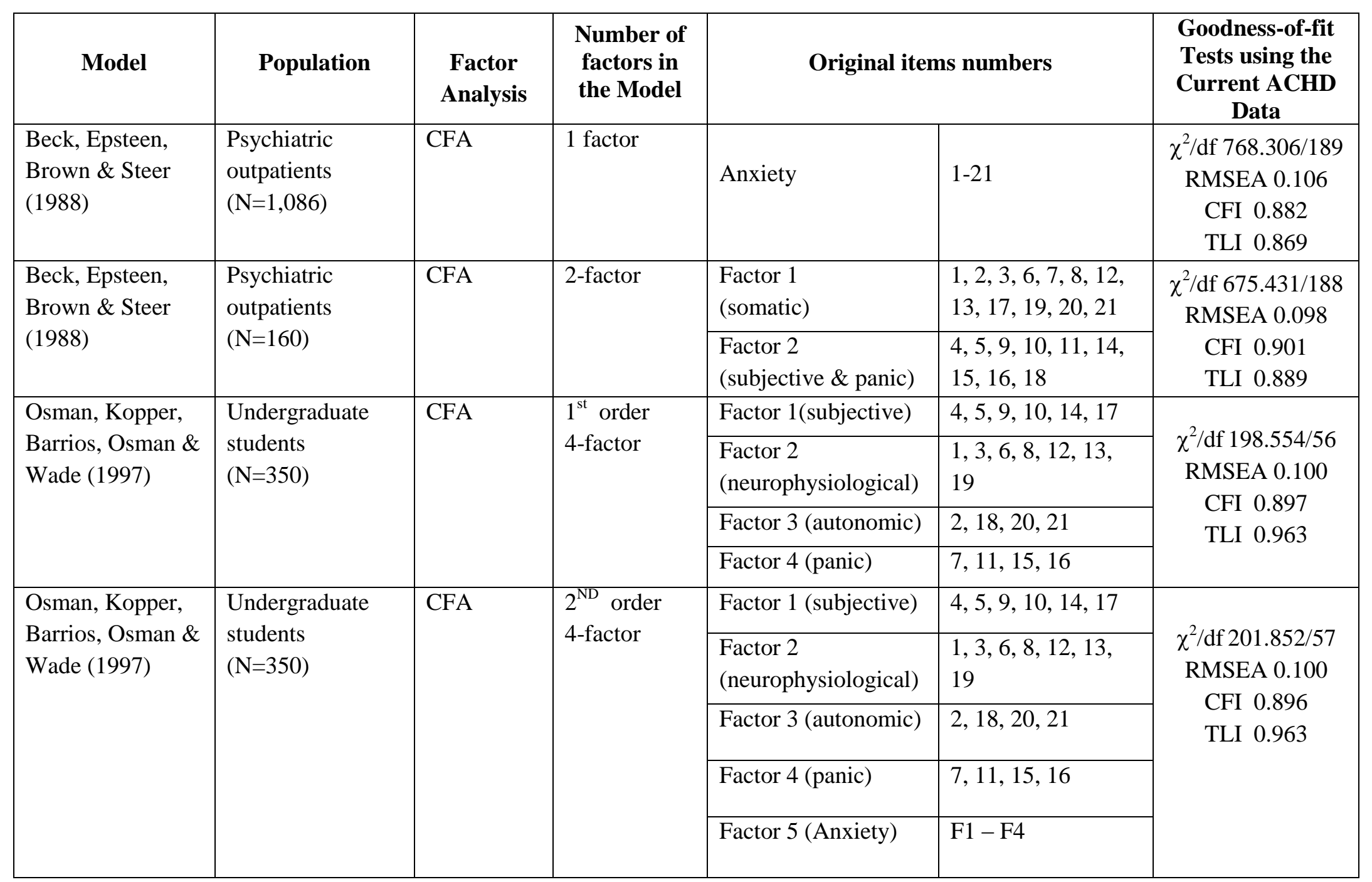


Table 4: $\quad$ Process of Selecting the Subjective Subscale of BAI Model Proposed by Osman et al. (1997)

\begin{tabular}{|c|c|c|c|c|c|}
\hline \multicolumn{2}{|c|}{ Model } & $\begin{array}{c}\text { Goodness-of-fit Tests } \\
\text { of the Model Using }\end{array}$ & \multicolumn{2}{|c|}{ Subscale used in this study } & $\begin{array}{l}\text { Goodness-of-fit } \\
\text { Tests of the } \\
\text { Subcale Usino the }\end{array}$ \\
\hline F1 (Subjective) & $4,5,9,10,14,17$ & \multirow{4}{*}{$\begin{array}{c}\chi^{2} / \mathrm{df} 373.806 / 185 \\
\text { RMSEA } 0.061 \\
\text { CFI } 0.962 \\
\text { TLI } 0.956\end{array}$} & \multirow{4}{*}{ F1* (Subjective) } & \multirow{4}{*}{$4,5,9,10,14,17$} & \multirow{4}{*}{$\begin{array}{c}\chi^{2} / \text { df } 20.150 / 9 \\
\text { RMSEA } 0.067 \\
\text { CFI } 0.994 \\
\text { TLI } 0.990\end{array}$} \\
\hline $\begin{array}{l}\text { F2 (Neuro- } \\
\text { Physiological) }\end{array}$ & $1,3,6,8,12,13,19$ & & & & \\
\hline F3 (Panic) & $2,18,20,21$ & & & & \\
\hline F4 (Autonomic) & $7,11,15,16$ & & & & \\
\hline
\end{tabular}

- Model proposed by Osman, Kopper, Barrios, Osman \& Wade (1997).

* F1 =Subjective factor (subscale) selected to represent anxiety as a single dimension in the current study. 
Figure 6: Proposed Measurement Model for BAI-Subjective Subscale to be Used for SEM in This Study

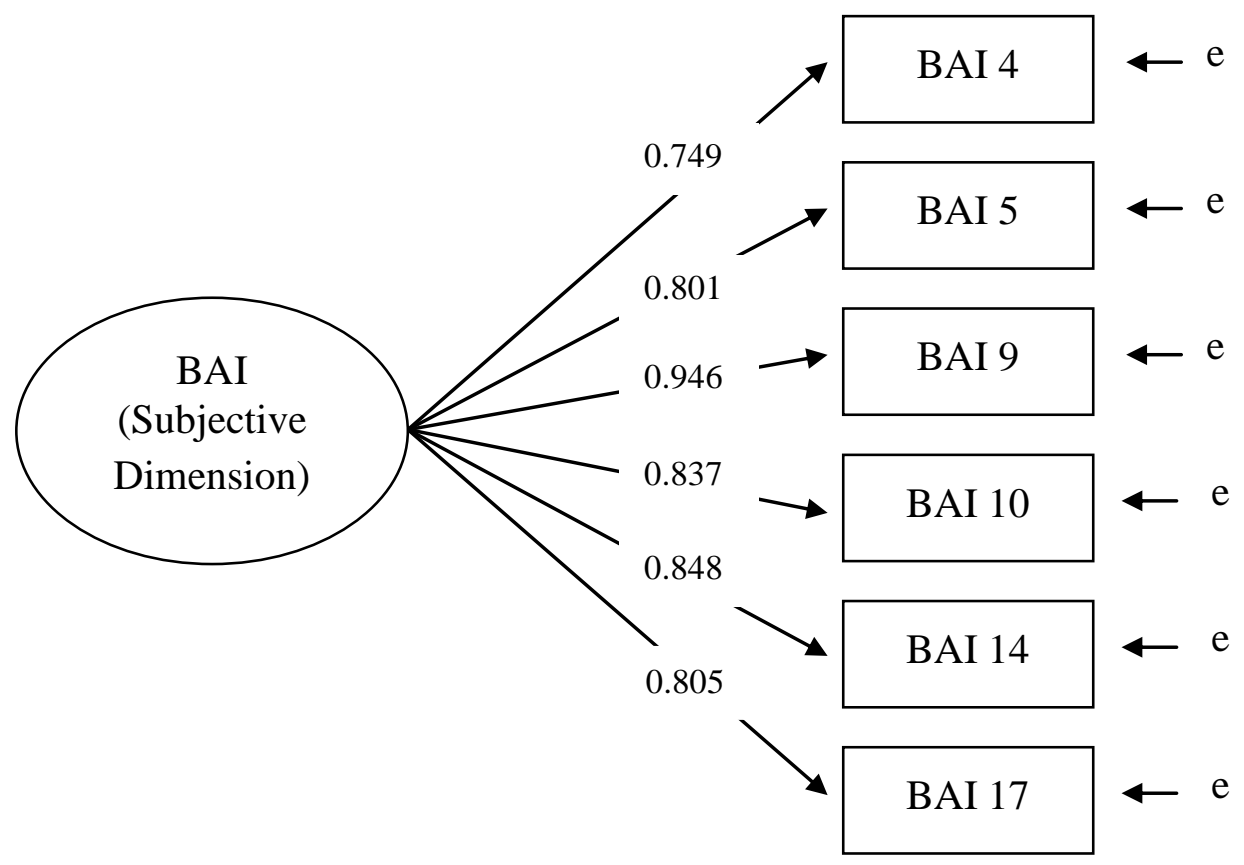

Values of factor loadings are the standardized estimates, all significant at $p<0.001$ Goodness-of-fit Indices: RMSEA 0.067 CFI 0.994

Thresholds were estimated but are not represented in this figure. 


\section{Beck Depression Inventory-II.}

In order to assist the reader in following the measurement assessment, a list of all 21 items and their corresponding item number can be found in Table 5. The determination of the factor structure of BDI-II for the current study was achieved by testing the fit of the different factor models previously reported (Table 6). The fit of both the one- and threefactor models (i.e., Osman et al's three-factor model) were poor. Of the two-factor models tested, the SA-C model (see page 57 for a detailed discussion) by Steer et al. (1999) demonstrated a poor fit to the data. The only model that demonstrated an acceptable fit was the two-factor model described by Whisman, Perez, \& Ramel (2000). The two factors of the model represented the Cognitive-Affective and Somatic dimensions. The fit indices

indicated that the model had a good fit with the data: $\chi^{2} 278.795 / 151$, CFI 0.970, TLI 0.966, RMSEA 0.056. Since the focus of this study was the cognitive-affective dimension of the depression, the cognitive symptoms need to be discriminated from the somatic symptoms of depression. Thus, the decision was made to retain Cognitive-Affective subscale of the BDIII. The factor structure of Cognitive-Affective subscale of the BDI-II is presented in Figure 7.

\section{Ways of Coping Questionnaire.}

The Ways of Coping Questionnaire (WCQ) is an instrument designed to assess coping as a process in accordance to the contextually oriented approach. The WCQ (1985), the revised version of the original Ways of Coping Checklist (WCCL), is currently one of the 


\section{Table 5: Items of the Beck Depression Inventory-II}

\begin{tabular}{|c|c|c|}
\hline $\begin{array}{c}\text { Item } \\
\text { number }\end{array}$ & Description & Example of a response \\
\hline 1 & Sadness & I feel sad much of the time. \\
\hline 2 & Pessimism & I feel more discouraged about my future than I used to be. \\
\hline 3 & Past Failure & I have failed more than I should have. \\
\hline 4 & Loss of Pleasure & I don't enjoy things as much as I used to. \\
\hline 5 & Guilty Feelings & $\begin{array}{l}\text { I feel guilty over many things I have done or should have } \\
\text { done. }\end{array}$ \\
\hline 6 & Punishment Feelings & I feel I may be punished. \\
\hline 7 & Self-Dislike & I have lost confidence in myself. \\
\hline 8 & Self-Criticalness & I am more critical of myself than I used to be. \\
\hline 9 & $\begin{array}{l}\text { Suicidal Thoughts or } \\
\text { Wishes }\end{array}$ & $\begin{array}{l}\text { I have thoughts of killing myself, but I would not carry } \\
\text { them out. }\end{array}$ \\
\hline 10 & Crying & I cry more than I used to. \\
\hline 11 & Agitation & I feel more restless or wound up than usual. \\
\hline 12 & Loss of Interest & I am less interested in other people or things than before. \\
\hline 13 & Indecisiveness & I find it more difficult to make decisions than usual. \\
\hline 14 & Worthlessness & $\begin{array}{l}\text { I don't consider myself as worthwhile and useful as I used } \\
\text { to. }\end{array}$ \\
\hline 15 & Loss of Energy & I have less energy than I used to have. \\
\hline 16 & Change in Sleeping Pattern & I sleep somewhat more than usual. \\
\hline 17 & Irritability & I am more irritable than usual. \\
\hline 18 & Change in Appetite & My appetite is somewhat less than usual. \\
\hline 19 & Concentration Difficulty & I can't concentrate as well as usual. \\
\hline 20 & Tiredness or Fatigue & I get more tired or fatigued more easily than usual. \\
\hline 21 & Loss of Interest in Sex & I am less interested in sex than I used to be. \\
\hline
\end{tabular}

See Appendix B for the complete BDI-II instrument. 
Table 6: Factor Models of BDI-II Described in the Literature

\begin{tabular}{|c|c|c|c|c|c|c|}
\hline Model & Population & EFA/CFA & Number of & \multicolumn{2}{|c|}{ Original items numbers } & $\begin{array}{l}\text { Goodness-of-fit } \\
\text { Tests using the }\end{array}$ \\
\hline \multirow[t]{2}{*}{$\begin{array}{l}\text { Arnau, Meagher, } \\
\text { Norris, \& } \\
\text { Bramson (2001) }\end{array}$} & \multirow[t]{2}{*}{$\begin{array}{l}\text { Patients from } \\
\text { medicine clinic } \\
(\mathrm{N}=333)\end{array}$} & \multirow[t]{2}{*}{$\begin{array}{l}\text { Principal } \\
\text { components } \\
\text { analysis }\end{array}$} & \multirow[t]{2}{*}{$\begin{array}{l}\text { 2-factor } \\
(\mathrm{SA}-\mathrm{C})\end{array}$} & $\begin{array}{l}\text { SA } \\
\text { (Somatic- } \\
\text { Affective) }\end{array}$ & $\begin{array}{l}1,4,11,12,13, \\
15,16,17,18,19, \\
20,21\end{array}$ & \multirow{2}{*}{$\begin{array}{l}\chi^{2} / \text { df } 139.501 / 59 \\
\text { RMSEA } 0.072 \\
\text { CFI } 0.953 \\
\text { TLI } 0.982\end{array}$} \\
\hline & & & & $\begin{array}{l}\mathbf{C} \\
\text { (Cognitive) }\end{array}$ & $\begin{array}{l}2,3,5,6,7,9,10, \\
14\end{array}$ & \\
\hline \multirow[t]{2}{*}{$\begin{array}{l}\text { Whisman, Perez, } \\
\text { \& Ramel (2000) }\end{array}$} & \multirow[t]{2}{*}{$\begin{array}{l}\text { Undergraduate } \\
\text { students } \\
(\mathrm{N}=576)\end{array}$} & \multirow[t]{2}{*}{ CFA } & \multirow[t]{2}{*}{$\begin{array}{l}\text { 2-factor } \\
\text { (CA-S) }\end{array}$} & $\begin{array}{l}\text { CA } \\
\text { (Cognitive- } \\
\text { Affective) }\end{array}$ & $\begin{array}{l}1,3,4,5,6,7,8 \\
9,10,11,12,13, \\
14,17\end{array}$ & \multirow{2}{*}{$\begin{array}{l}\chi^{2} / \text { df } 278.795 / 151 \\
\text { RMSEA } 0.056 \\
\text { CFI } 0.970 \\
\text { TLI } 0.966\end{array}$} \\
\hline & & & & $\begin{array}{l}\text { S } \\
\text { (Somatic) }\end{array}$ & $15,16,18,19,20$ & \\
\hline $\begin{array}{l}\text { Whisman, Perez, } \\
\text { \& Ramel (2000) } \\
\text { Note: this is the same } \\
\text { study as above }\end{array}$ & Same as above & $\begin{array}{l}\text { Same as } \\
\text { above }\end{array}$ & $\begin{array}{l}\text { only } \\
\text { (CA) }\end{array}$ & $\begin{array}{l}\text { CA* } \\
\text { (Cognitive- } \\
\text { Affective) }\end{array}$ & $\begin{array}{l}1,3,4,5,6,7,8 \\
9,10,11,12,13 \\
14,17\end{array}$ & $\begin{array}{l}\chi^{2} / \mathrm{df} 136.927 / 77 \\
\text { RMSEA } 0.053 \\
\text { CFI } 0.979 \\
\text { TLI } 0.975\end{array}$ \\
\hline
\end{tabular}

Note: The BDI-II, which consists of 21 items, can be divided into two subscales that assess different dimensions of depression: cognitive-affective domain and somatic-performance domain. Cognitive-affective domain measures cognitively oriented symptoms, e.g., mood, suicidal thoughts, while somatic performance domain measures non-cognitive symptoms, e.g., crying, loss of energy.

A slight variation of factor composition exists in the two-factor model attributed to the salient loadings in each of the factors. The first variation of the model is labelled somatic-affective and cognitive (SA-C) and the second variation of the model is labelled cognitive-affective and somatic (CA-S).

* shaded cell represents the Cognitive-Affective factor and item composition that was selected to represent depression as a single dimension in this study. 
Figure 7: Proposed Measurement Model for Cognitive-Affective Subscale of the BDI-II to be Used for SEM in This Study

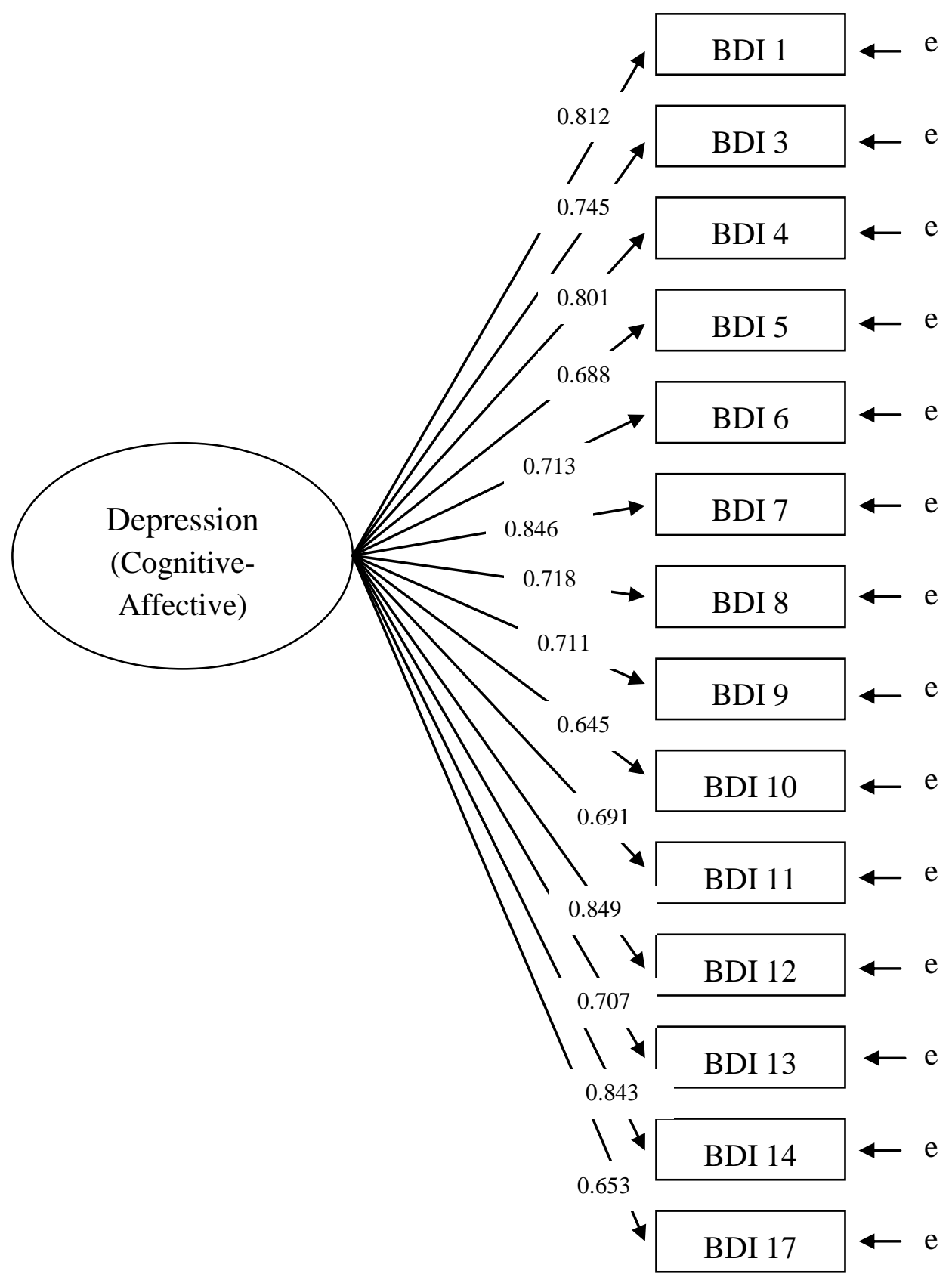

Goodness-of-fit Indices: RMSEA 0.053 CFI 0.979

Values of factor loadings are the standardized estimates, all significant at $p<0.001$ 
most widely used self-reporting measures for examining coping from the contextual (process) approach (Chan, 1994). Scherer, Wiebe, Luther and Adams (1988) have noted that a critical issue facing the contextually oriented approach is the stability of the measure. They claim that the stability of a measure, i.e., factor structure, must be established in order to test a theory and to make a meaningful interpretation of the results. Construct validity must be obtained by reproducing factors across studies and ascertaining whether the hypothesized factor structure is supported (Comrey, 1978). However, the performance of the WCQ as a stable measure of coping has been problematic due to the inconsistent psychometric properties that have frequently been reported (Bouchard, Sabourin, Lussier, Wright \& Richer, 1997; Parker \& Endler, 1992). More specifically, the findings of both the exploratory factor analyses (EFA) and confirmatory factor analyses (CFA) vary considerably, and few identified factor structures have been cross-validated (Parker, Endler \& Bagby, 1993; Scherer, Wiebe, Luther \& Adams, 1988). For example, the original EFA model of the WCQ proposed by Lazarus and Folkman (1985) was an eight-factor model. Since then, EFA and CFA factors have been extracted from the WCQ including, a three-factor model (McColl \& Skinner, 1995; Wineman, Durand \& McCullouch , 1994); a four-factor model (Bouchard et al., 1997; Chan (1994); Parker, Endler, \& Bagby, 1993; Yip, Rowlinson, \& Siu , 2008); a five-factor model (Scherer, Wiebe, Luther, \& Adams, 1988; Sorlie \& Sexton, 2001; sixfactor model (Munet-Vilaro, Gregorich, \& Folkman, 2002), and an eight-factor model (Atkinson \& Violato, 1993; Folkman, Lazarus, Dunkel-Schetter, DeLongis, \& Gruen, 1986) (Table 7) . Furthermore, the item composition of the scales with similar names to those initially identified in studies by Folkman and colleagues (1988) also vary across studies 
(Chan, 1994; Edwards \& O’Neill, 1998). For instance, the item composition of the three subscales identified in Folkman et al.'s (1985) study included: problem-focused (2, 26, 35, $39,46,48,49,52,54,62,64)$, detachment $(4,12,13,21,24,53)$, and wishful thinking (11, $55,57,58,59)$. However, the item composition of the similar subscales in Chan's (1994) study was named: problem-focused $(1,2,23,24,26,39,48,49,52,54,56,61,62,64)$, distancing $(12,13,21,32,41)$, and wishful thinking $(55,57,58,59)$. Such a pattern of dissimilar item composition for similarly named factors is frequently observed across the studies that have attempted to analyze the factor structures of the WCQ. In addition, the confirmatory factor analyses attempting to generalize the previously identified factor structures showed poor fit indices and failed to validate the structure (Edwards \& O'Neil, 1998; Parker et al., 1993; Sorlie \& Sexton, 2001).

Similar to previous studies that failed to validate factor structures of the WCQ, the current study was also unsuccessful in confirming previously identified factor structures. As indicated in Table 7, regardless of the number of factors, the fit indices of confirmatory factor analyses demonstrated that none of the exploratory factor structures had a good fit with the data from the participants of this study. The failure to replicate the existing factor structures resonates with the statement made by Parker et al. (1993) who noted, “investigators using the WCQ measure have frequently found a different number of factors depending on the sample and the particular number of items selected" (p. 333). Subsequently, they make a recommendation to researchers to conduct the factor analysis of the WCQ with their own study samples. Thus, the decision was made to carry out an EFA 
Table 7: Factor Models of the Ways of Coping Questionnaire Described in the Literature

\begin{tabular}{|c|c|c|c|c|c|c|}
\hline Model & Population & EFA/CFA & Number of & \multicolumn{2}{|c|}{ Original items numbers } & $\begin{array}{l}\text { Fit indices of } \\
3 \text { selected }\end{array}$ \\
\hline \multirow{6}{*}{$\begin{array}{l}\text { Munet-Vilaro, } \\
\text { Gregorich, \& } \\
\text { Folkman (2002) }\end{array}$} & \multirow{6}{*}{$\begin{array}{l}\text { Latinos from } \\
\text { Mexico City, } \\
\text { Puerto Rico, and } \\
\text { San Francisco } \\
\text { Peninsula } \\
(\mathrm{N}=1063)\end{array}$} & \multirow[t]{6}{*}{ EFA } & \multirow[t]{6}{*}{ 6-factor model } & Problem -Solving & $1,5,20,26,49$ & \multirow{6}{*}{$\begin{array}{lc}3 \text {-factor model } \\
\chi^{2} / \mathrm{df} & 408.032 / 27 \\
\text { RMSEA } & 0.162 \\
\text { CFI } & 0.746 \\
\text { TLI } & 0.718\end{array}$} \\
\hline & & & & Social support & $8,22,31,42,45$ & \\
\hline & & & & $\begin{array}{l}\text { Cognitive Escape/ } \\
\text { Avoidance }\end{array}$ & $55,57,58,59$ & \\
\hline & & & & Distancing & $13,21,41,44$ & \\
\hline & & & & $\begin{array}{l}\text { Accepting } \\
\text { Responsibility }\end{array}$ & $9,25,29,51$ & \\
\hline & & & & Confrontive- Coping & 7,17 & \\
\hline \multirow{3}{*}{$\begin{array}{l}\text { Folkman, } \\
\text { Lazarus, } \\
\text { Dunkel- } \\
\text { Schetter, } \\
\text { DeLongis, \& } \\
\text { Gruen (1986) }\end{array}$} & \multirow{3}{*}{$\begin{array}{l}\text { Community- } \\
\text { residing married } \\
\text { couples } \\
(\mathrm{N}=85 \text { couples })\end{array}$} & \multirow[t]{3}{*}{ EFA } & \multirow[t]{3}{*}{8 -factor model } & Confrontive- Coping & $\begin{array}{l}6,7,17,28,34, \\
46\end{array}$ & \multirow{3}{*}{$\begin{array}{l}\text { 3-factor model } \\
\chi^{2} / \mathrm{df} \\
505.851 / 167 \\
\text { RMSEA } 0.086 \\
\text { CFI } 0.812 \\
\text { TLI } 0.786\end{array}$} \\
\hline & & & & Distancing & $\begin{array}{l}12,13,15,21,41 \\
44\end{array}$ & \\
\hline & & & & Self-Controlling & $\begin{array}{l}10,14,35,43,54 \\
62,63\end{array}$ & \\
\hline
\end{tabular}

Note: Items included in the shaded subscales are the factors used in the goodness-of-fit testing involving this study's ACHD data 
Table 7: Factor Models of the Ways of Coping Questionnaire Described in the Literature (continued)

\begin{tabular}{|c|c|c|c|c|c|c|}
\hline \multirow[t]{6}{*}{ Model } & \multirow[t]{6}{*}{ Population } & \multirow[t]{6}{*}{ EFA/CFA } & \multirow[t]{6}{*}{$\begin{array}{l}\text { Number of } \\
\text { factors }\end{array}$} & \multicolumn{2}{|c|}{ Original items numbers } & \multirow{6}{*}{\begin{tabular}{|c|} 
Fit indices of \\
3 selected \\
subscales \\
using the \\
current ACHD \\
data \\
\end{tabular}} \\
\hline & & & & $\begin{array}{l}\text { Seeking Social } \\
\text { Support }\end{array}$ & $\begin{array}{l}8,18,22,31,42, \\
45\end{array}$ & \\
\hline & & & & $\begin{array}{l}\text { Accepting } \\
\text { Responsibility }\end{array}$ & $9,25,29,51$ & \\
\hline & & & & Escape-Avoidance & $\begin{array}{l}11,16,33,40,47, \\
50,58,59\end{array}$ & \\
\hline & & & & $\begin{array}{l}\text { Planful Problem- } \\
\text { solving }\end{array}$ & $\begin{array}{l}1,26,39,48,49, \\
52\end{array}$ & \\
\hline & & & & Positive Reappraisal & $\begin{array}{l}20,23,30,36,38 \\
56,60\end{array}$ & \\
\hline \multirow[t]{5}{*}{$\begin{array}{l}\text { Scherer, Wiebe, } \\
\text { Luther, \& Adams } \\
\text { (1988) }\end{array}$} & \multirow[t]{5}{*}{$\begin{array}{l}\text { Undergraduate } \\
\text { university students } \\
(\mathrm{N}=491)\end{array}$} & \multirow[t]{5}{*}{ EFA } & \multirow[t]{5}{*}{ 5-factor model } & $\begin{array}{l}\text { Problem-focused } \\
\text { Coping }\end{array}$ & $\begin{array}{l}2,26,35,39,48 \\
49,52,54,62,64\end{array}$ & \multirow{5}{*}{\begin{tabular}{ll}
\multicolumn{2}{c}{3 -factor model } \\
$\chi^{2} / \mathrm{df}$ & \\
$457.407 / 186$ \\
RMSEA & 0.073 \\
CFI & 0.900 \\
TLI & 0.887
\end{tabular}} \\
\hline & & & & Detachment & $\begin{array}{l}4,12,13,21,24, \\
53\end{array}$ & \\
\hline & & & & Wishful Thinking & $11,55,57,58,59$ & \\
\hline & & & & $\begin{array}{l}\text { Seeking Social } \\
\text { Support }\end{array}$ & $8,18,28,42,45$ & \\
\hline & & & & $\begin{array}{l}\text { Focusing on the } \\
\text { Positive }\end{array}$ & $15,20,23,38$ & \\
\hline
\end{tabular}

Note: Items included in the shaded subscales are the factors used in the goodness-of-fit testing involving this study's ACHD data 
Table 7: Factor Models of the Ways of Coping Questionnaire Described in the Literature (continued)

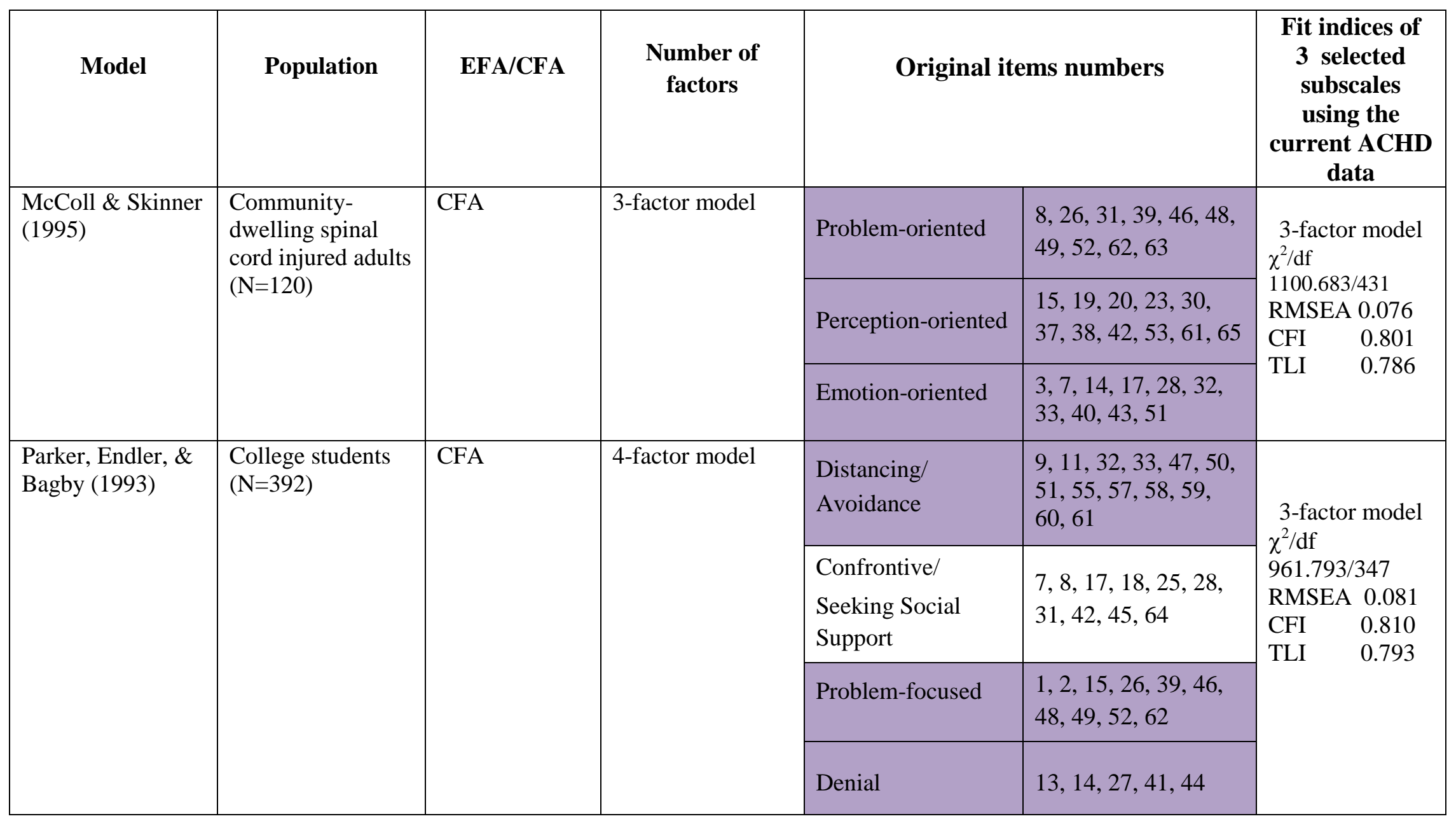

Note: Items included in the shaded subscales are the factors used in the goodness-of-fit testing involving this study's ACHD data 
Table 7: Factor Models of the Ways of Coping Questionnaire Described in the Literature (continued)

\begin{tabular}{|c|c|c|c|c|c|c|}
\hline Model & Population & EFA/CFA & Number of & \multicolumn{2}{|c|}{ Original items numbers } & $\begin{array}{c}\text { Fit indices of } 3 \\
\text { selected subscales }\end{array}$ \\
\hline \multirow[t]{5}{*}{$\begin{array}{l}\text { Sorlie \& Sexton } \\
\text { (2001) }\end{array}$} & \multirow{5}{*}{$\begin{array}{l}\text { Adults treated } \\
\text { with urological, } \\
\text { cardiac and } \\
\text { gastrointestinal } \\
\text { surgeries } \\
(\mathrm{N}=555)\end{array}$} & \multirow[t]{5}{*}{ EFA } & \multirow[t]{5}{*}{ 5-factor model } & Wishful Thinking & $11,12,57,58,59,61$ & \multirow{5}{*}{\begin{tabular}{lc}
\multicolumn{2}{r}{ 3-factor model } \\
$\chi^{2} / \mathrm{df}$ & $351.116 / 101$ \\
RMSEA & 0.095 \\
CFI & 0.871 \\
TLI & 0.847
\end{tabular}} \\
\hline & & & & Goal Oriented & $1,2,5,15,19$ & \\
\hline & & & & Seeking Support & $8,22,31,42,45$ & \\
\hline & & & & Thinking it over & $48,49,51,52$ & \\
\hline & & & & Avoidance & $14,37,41,43,44,54$ & \\
\hline \multirow[t]{6}{*}{$\begin{array}{l}\text { Atkinson \& } \\
\text { Violato (1993) }\end{array}$} & \multirow[t]{6}{*}{$\begin{array}{l}\text { Second-year } \\
\text { university } \\
\text { students }(\mathrm{N}=149)\end{array}$} & \multirow[t]{6}{*}{ EFA } & \multirow[t]{6}{*}{8 -factor model } & Problem-solving & $\begin{array}{l}1,10,20,26,35,39 \\
48,49,52,62,63\end{array}$ & \multirow{6}{*}{\begin{tabular}{|ll} 
3-factor model \\
$\chi^{2} /$ df & $702.377 / 249$ \\
RMSEA & 0.082 \\
CFI & 0.793 \\
TLI & 0.770
\end{tabular}} \\
\hline & & & & $\begin{array}{l}\text { Seeking Social } \\
\text { support }\end{array}$ & $8,18,28,42,45$ & \\
\hline & & & & Positive reappraisal & $23,30,36,38,56,60$ & \\
\hline & & & & Emotional distancing & $\begin{array}{l}13,14,15,21,41,44, \\
54\end{array}$ & \\
\hline & & & & Confrontive Coping & $7,17,31,34,46,47$ & \\
\hline & & & & $\begin{array}{l}\text { Accepting } \\
\text { responsibility }\end{array}$ & $9,25,29,51$ & \\
\hline
\end{tabular}

Note: Items included in the shaded subscales are the factors used in the goodness-of-fit testing involving this study's ACHD data 
Table 7: Factor Models of the Ways of Coping Questionnaire Described in the Literature (continued)

\begin{tabular}{|c|c|c|c|c|c|c|}
\hline \multirow[t]{3}{*}{ Model } & \multirow[t]{3}{*}{ Population } & \multirow[t]{3}{*}{ EFA/CFA } & \multirow[t]{3}{*}{$\begin{array}{l}\text { Number of } \\
\text { factors }\end{array}$} & \multicolumn{2}{|c|}{ Original items numbers } & \multirow[t]{3}{*}{$\begin{array}{c}\text { Fit indices of } \\
3 \text { selected } \\
\text { subscales using } \\
\text { the current } \\
\text { ACHD data } \\
\end{array}$} \\
\hline & & & & $\begin{array}{l}\text { Escape-Avoidance } \\
\text { (cognitive) }\end{array}$ & $6,11,12,50,58,59$ & \\
\hline & & & & $\begin{array}{l}\text { Escape-Avoidance } \\
\text { (physiological) }\end{array}$ & $16,22,33,40,43$ & \\
\hline \multirow[t]{4}{*}{$\begin{array}{l}\text { Bouchard et al. } \\
\text { (1997) }\end{array}$} & \multirow[t]{4}{*}{$\begin{array}{l}\text { French } \\
\text { Canadian } \\
\text { couples } \\
(\mathrm{N}=506)\end{array}$} & \multirow[t]{4}{*}{ CFA } & \multirow[t]{4}{*}{ 4-factor model } & Distancing & $\begin{array}{l}9,11,32,33,47,50 \\
51,55,57,58,59 \\
60,61\end{array}$ & \multirow{4}{*}{$\begin{array}{ll}3 \text {-factor model } \\
\chi^{2} / \mathrm{df} & 434.276 / 50 \\
\text { RMSEA } & 0.131 \\
\text { CFI } & 0.700 \\
\text { TLI } & 0.711\end{array}$} \\
\hline & & & & $\begin{array}{l}\text { Confrontation/ } \\
\text { Seeking Social } \\
\text { Support }\end{array}$ & $\begin{array}{l}7,8,17,18,25,28 \\
31,42,45,64\end{array}$ & \\
\hline & & & & Problem-focused & $\begin{array}{l}1,2,15,26,39,46, \\
48,49,52,62\end{array}$ & \\
\hline & & & & Denial & $13,14,27,41,44$ & \\
\hline \multirow[t]{2}{*}{ Chan (1994) } & \multirow{2}{*}{$\begin{array}{l}\text { Chinese } \\
\text { secondary } \\
\text { school students } \\
\text { and teachers } \\
(\mathrm{N}=657)\end{array}$} & \multirow[t]{2}{*}{$\begin{array}{l}\text { EFA } \\
\text { WCQ } \\
\text { Chinese Version }\end{array}$} & 4-factor model & Problem solving & $\begin{array}{l}1,2,23,24,26,39 \\
48,49,52,54,56 \\
61,62,64\end{array}$ & \multirow{2}{*}{$\begin{array}{lc}3 \text {-factor model } \\
\chi^{2} / \text { df } & 759.926 / 227 \\
\text { RMSEA } & 0.093 \\
\text { CFI } & 0.824 \\
\text { TLI } & 0.804\end{array}$} \\
\hline & & & & $\begin{array}{l}\text { Resigned } \\
\text { distancing }\end{array}$ & $12,13,21,32,41$ & \\
\hline
\end{tabular}

Note: Items included in the shaded subscales are the factors used in the goodness-of-fit testing involving this study's ACHD data 
Table 7: Factor Models of the Ways of Coping Questionnaire Described in the Literature (continued)

\begin{tabular}{|c|c|c|c|c|c|c|}
\hline \multirow{3}{*}{ Model } & \multirow{3}{*}{ Population } & \multirow{3}{*}{ EFA/CFA } & \multirow{3}{*}{$\begin{array}{l}\text { Number of } \\
\text { factors }\end{array}$} & \multicolumn{2}{|c|}{ Original items numbers } & \multirow{3}{*}{$\begin{array}{l}\text { Fit indices of } 3 \\
\text { selected subscales } \\
\text { using the current } \\
\text { ACHD data }\end{array}$} \\
\hline & & & & Seeking support & $8,28,31,42,45,47$ & \\
\hline & & & & $\begin{array}{l}\text { Passive wishful } \\
\text { thinking }\end{array}$ & $55,57,58,59$ & \\
\hline \multirow{3}{*}{$\begin{array}{l}\text { Wineman, } \\
\text { Durand \& } \\
\text { McCullouch } \\
(1994)\end{array}$} & \multirow{3}{*}{$\begin{array}{l}\text { Community } \\
\text { residing adults } \\
\text { with multiple } \\
\text { sclerosis or a } \\
\text { spinal cord } \\
\text { injury } \\
(\mathrm{N}=437)\end{array}$} & \multirow[t]{3}{*}{ CFA } & \multirow[t]{3}{*}{ 3-factor model } & $\begin{array}{l}\text { Cognitive } \\
\text { reframing }\end{array}$ & $\begin{array}{l}10,15,19,20,23 \\
30,52\end{array}$ & \multirow{3}{*}{\begin{tabular}{lc}
\multicolumn{2}{c}{ 3-factor model } \\
$\chi^{2} / \mathrm{df}$ & $156.373 / 62$ \\
RMSEA & 0.075 \\
CFI & 0.947 \\
TLI & 0.933
\end{tabular}} \\
\hline & & & & Emotional respite & $11,55,57,59$ & \\
\hline & & & & Direct assistance & $31,42,45$ & \\
\hline \multirow[t]{3}{*}{$\begin{array}{l}\text { Yip, Rowlinson, } \\
\text { \& Siu } \\
(2008)\end{array}$} & \multirow{3}{*}{$\begin{array}{l}\text { Professional } \\
\text { engineers in the } \\
\text { construction } \\
\text { industry } \\
(\mathrm{N}=222)\end{array}$} & \multirow{3}{*}{$\begin{array}{l}\text { CFA } \\
\text { WCQ } \\
\text { Revised scale } \\
\text { suggested by } \\
\text { Chan (1994) }\end{array}$} & \multirow[t]{3}{*}{ 4-factor model } & Problem solving & $\begin{array}{l}1,2,23,24,26,39 \\
48,49,52,54,56 \\
61,62,64\end{array}$ & \multirow{3}{*}{$\begin{array}{l}3 \text {-factor model } \\
\chi^{2} / \mathrm{df} \quad 759.926 / 227 \\
\text { RMSEA } 0.093 \\
\text { CFI } 0.824 \\
\text { TLI } 0.804\end{array}$} \\
\hline & & & & $\begin{array}{l}\text { Resigned } \\
\text { distancing }\end{array}$ & $12,13,21,32,41$ & \\
\hline & & & & Seeking support & $8,28,31,42,45,47$ & \\
\hline
\end{tabular}

Note: Items included in the shaded subscales are the factors used in the goodness-of-fit testing involving this study's ACHD data 
Table 7: Factor Models of the Ways of Coping Questionnaire Described in the Literature (continued)

\begin{tabular}{|l|l|l|l|l|l|l|}
\hline Model & Population & EFA/CFA & $\begin{array}{c}\text { Fumber of } \\
\text { factors }\end{array}$ & \multicolumn{2}{|c|}{$\begin{array}{c}\text { Original items numbers } \\
3 \\
\text { subscales } \\
\text { the current } \\
\text { ACHD data }\end{array}$} \\
\hline & & & & $\begin{array}{l}\text { Passive wishful } \\
\text { thinking }\end{array}$ & $55,57,58,59$ \\
\hline
\end{tabular}

Note: Items included in the shaded subscales are the factors used in the goodness-of-fit testing involving this study's ACHD data 
of the WCQ to determine the factor structure that represents data from the participants of this study with congenital heart disease.

The EFA of the WCQ was performed with all of its 66 items to delineate the factor patterns. The geomin oblique rotation was used to analyze the data. Cases with missing values were excluded with the pairwise deletion. The EFA was performed with 66 items, from which a minimum of one factor and a maximum of eight factors were allowed to be extracted. The Cattell's scree plot of the eigenvalues computed is presented in Figure 8. 18 factors displayed an eigenvalue of greater than 1 (Kaiser criterion). However, the scree plot was not helpful for deciding the number of factors to retain. Not only can the selection of factors based on eigenvalues lead to arbitrary decisions and overestimation of the number of factors (Zwick \& Velicer, 1986), retaining the 18 factors was an unrealistic and impractical goal for the study. Therefore, the decision was made to choose the range of one to eight factors based on the theory and previous research on the total number of factors that are actually present in the scale. The evidence from different studies seems to indicate that a number of factors identified vary widely from two to eight. Thus, it was decided to extract the entire range of number of factors that have been identified thus far across the studies. For each of one to eight factors, the Mplus program provided the goodness-of-fit indices, including $\chi^{2}$, RMSEA and CFI to assess the fit of the model. The examination of the EFA result showed the improvement in fit indices (i.e., RMSEA and CFI) as the number of factors increased. The pattern of factor loading was similar between six- and eight factor models. However, at the level of seven- and eight- factor models, the number of items with 


\section{Figure 8: The Scree Plot of Eigenvalues Extracted From the Ways of Coping Questionnaire}

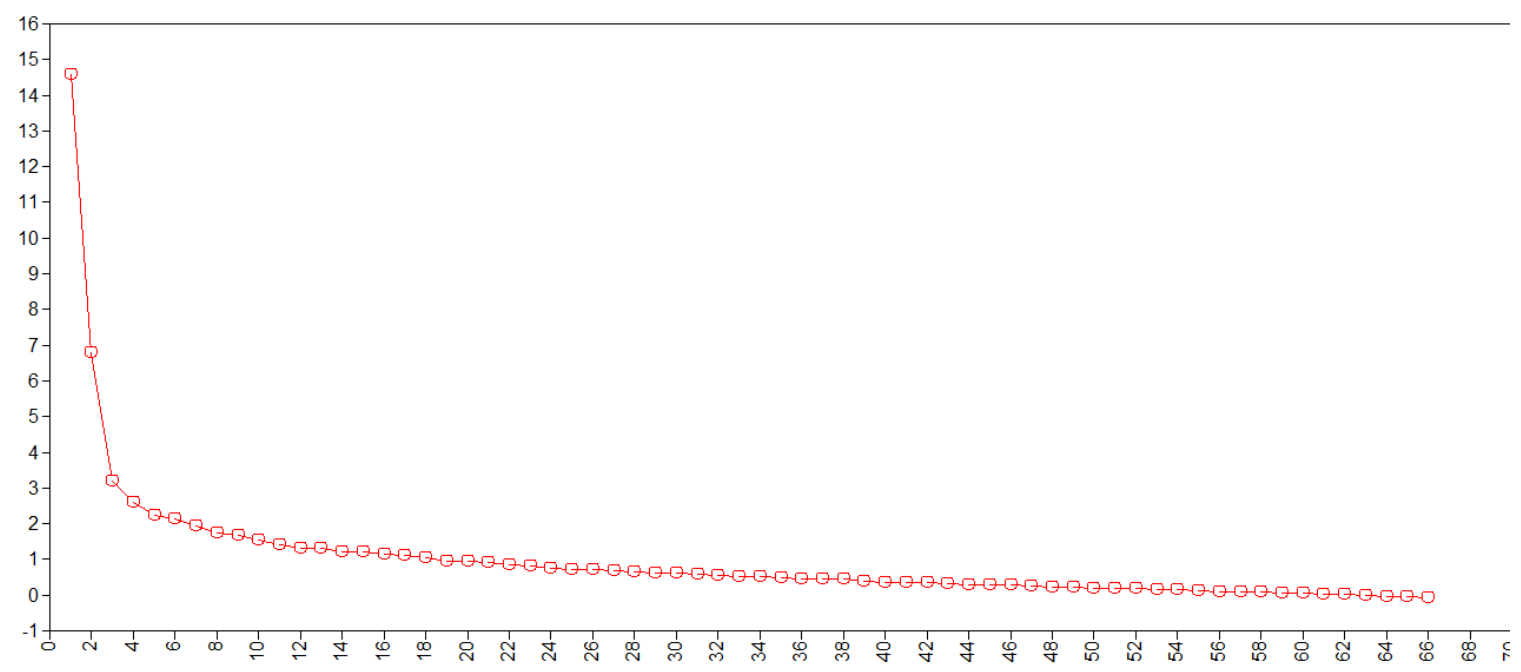

significant factor loadings (i.e., $\geq .4$ ) decreased substantially. In fact, for the eight-factor model, only one item had a significant factor loading. Furthermore, for factors greater than six, the substantive meaning of a couple of latent factors decreased substantially. However, with the six-factor model, more items with significant factor loadings were consistently loading on factors. Thus, the six-factor model was retained for the purpose of computing three subscales required for the analyses.

The three subscales extracted from the six-factor EFA model were: problem-solving, wishful-thinking, and denial/avoidance coping strategies (Figure 9). The items for three subscales, their factors loadings, and fit indices of the three-factor EFA model is presented in Table 8. The goodness-of-fit indices of three subscales were poor: $\chi^{2}(294)=800.804$, 
Table 8: The Factor Structure of the Three Subscales of Ways of Coping Questionnaire Established With the Data From the Current Study

\begin{tabular}{|c|c|c|c|c|}
\hline $\begin{array}{c}\text { Item } \\
\text { number }\end{array}$ & Item description & $\begin{array}{c}\text { Factor } 1 \\
\text { Problem- } \\
\text { solving }\end{array}$ & $\begin{array}{l}\text { Factor } 2 \\
\text { Wishful- } \\
\text { thinking }\end{array}$ & $\begin{array}{c}\text { Factor } 3 \\
\text { Denial/ } \\
\text { Avoidance }\end{array}$ \\
\hline 1 & Just concentrated on what I had to do next - the next step. & 0.460 & & \\
\hline 2 & I tried to analyze the problem in order to understand it better. & 0.471 & & \\
\hline 4 & I felt that time would make a difference - the only thing to do was to wait. & & & 0.458 \\
\hline 5 & Bargained or compromised to get something positive from the situation.* & 0.433 & & \\
\hline 8 & Talked to someone to find out more about the situation.* & 0.414 & & \\
\hline 11 & Hoped a miracle would happen. & & 0.650 & \\
\hline 12 & Went along with fate; sometimes I just have bad luck. & & & 0.416 \\
\hline 13 & Went on as if nothing had happened. & & & 0.528 \\
\hline 15 & Looked for silver lining, so to speak; tried to look on the bright side of things. & & & 0.557 \\
\hline 21 & Tried to forget the whole thing.* & -0.403 & & 0.453 \\
\hline 26 & I made a plan of action and followed it. & 0.590 & & \\
\hline 31 & Talked to someone who could do something concrete about the problem. & 0.454 & & \\
\hline 40 & Avoided being with people in general. & & 0.458 & \\
\hline 41 & Didn`t let it get to me; refused to think too much about it. & & & 0.467 \\
\hline 44 & Made light of the situation; refused to get too serious about it. & & & 0.539 \\
\hline 49 & Drew on my past experiences; I was in a similar situation before. & 0.527 & & (continued) \\
\hline
\end{tabular}


Table 8: The Factor Structure of the Three Subscales of Ways of Coping Questionnaire Established With the Data From Current Study (continued)

\begin{tabular}{|c|c|c|c|c|}
\hline $\begin{array}{c}\text { Item } \\
\text { Number }\end{array}$ & Item Description & $\begin{array}{c}\text { Factor } 1 \\
\text { Problem- } \\
\text { solving }\end{array}$ & $\begin{array}{l}\text { Factor } 2 \\
\text { Wishful- } \\
\text { thinking }\end{array}$ & $\begin{array}{c}\text { Factor } 3 \\
\text { Denial/ } \\
\text { Avoidance }\end{array}$ \\
\hline 52 & Came up with a couple of different solutions to the problem. & 0.588 & & \\
\hline 53 & Accepted it, since nothing could be done. & & & 0.516 \\
\hline 54 & I tried to keep my feelings from interfering with other things too much. & & & 0.413 \\
\hline 55 & I tried to keep my feelings from interfering with other things too much. & & 0.738 & \\
\hline 57 & I daydreamed or imagined a better time or place than the one I was in. & & 0.737 & \\
\hline 58 & Wished that the situation would go away or somehow be over with. & & 0.776 & \\
\hline 59 & Had fantasies or wishes about how things might turn out. & & 0.715 & \\
\hline 61 & I prepared myself for the worst. & & 0.502 & \\
\hline 62 & $\begin{array}{l}\text { I thought about how a person I admire would handle this situation and used } \\
\text { that as a model.* }\end{array}$ & 0.411 & 0.420 & \\
\hline 64 & I jogged or exercised. & 0.404 & & \\
\hline
\end{tabular}

* These items loaded to more than one factor.

Fit indices of 3-factor model: $\chi^{2}=295.103 d f=165$ RMSEA 0.054 CFI 0.948 
Figure 9: Proposed Measurement Model of Three Subscales of WCQ - Problem-Solving, Wishful-Thinking, Denial/Avoidance - to be Used in This Study With the ACHD Data

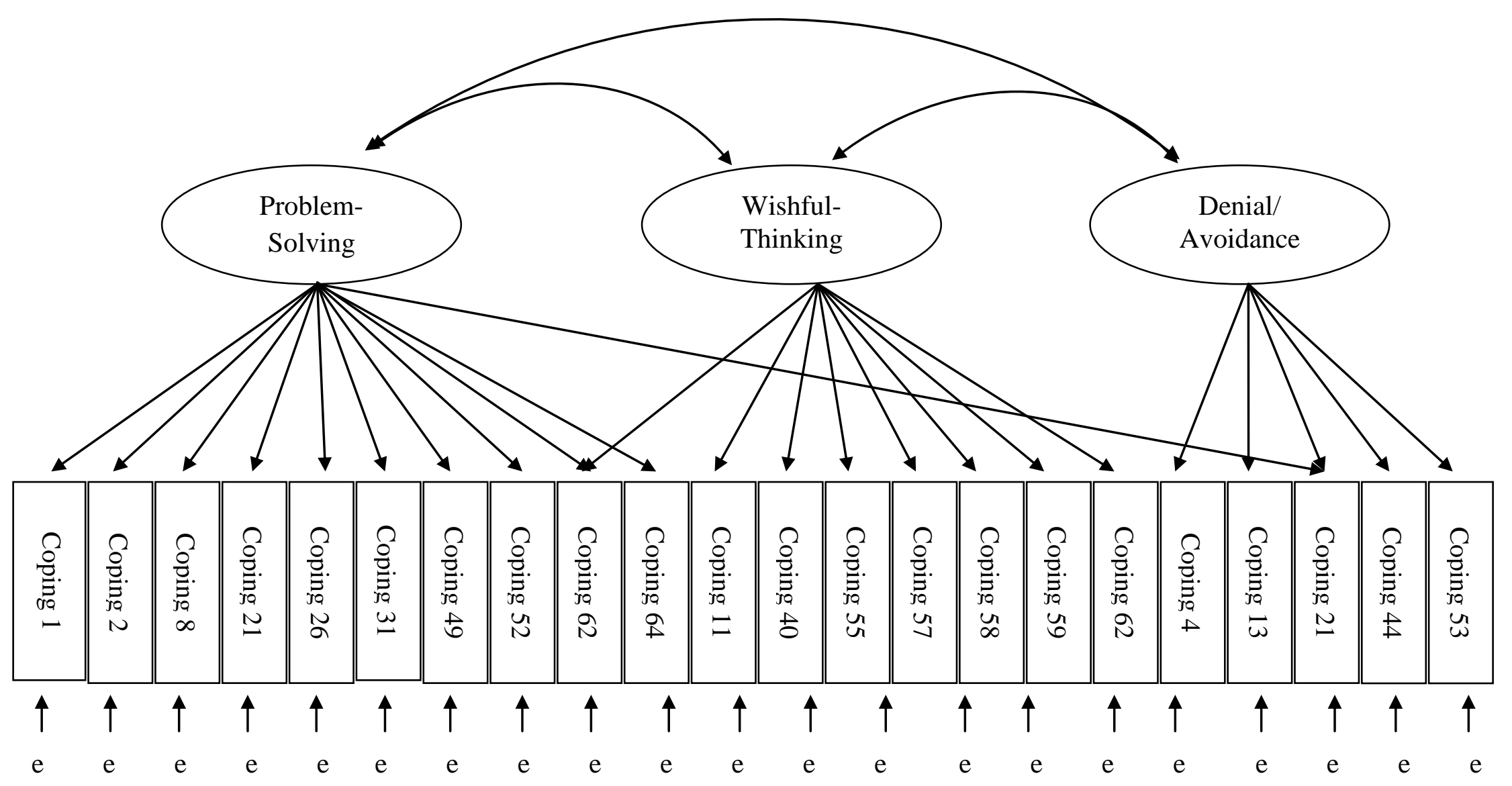


RMSEA $0.08, \mathrm{CFI} 0.84$. The evaluation of the model indicated that a number of items were associated with high residuals and modification indices (MI): item number 61 (I prepared myself for the worse), 12 (Went along with fate; sometimes I just have bad luck), 15 (Looked for silver lining, so to speak; tried to look on the bright side of things), 41 (Didn't let it get to me; refused to think too much about it), and 5 (Bargained or compromised to get something positive from the situation). Based on the model fit and evaluation indices (i.e., residual, and MI), items were eliminated one at a time, and fit of the model was tested (i.e., RMSEA, CFI) following an elimination of each item. The residuals and the range of MIs associated with each item were: $61(0.24,105), 12(0.27,58), 15(0.26,33), 41(0.21,34)$, and $5(0.19,20)$. Following the elimination of five ill-fitting items, a total of 22 items comprised the three subscales of coping strategies: problem-solving (10 items), wishful-thinking (7 items), and denial/avoidance ( 5 items). Of the 22 items of three subscales, item number 62 (I went over in my mind what I would say or do) and 21 (Tried to forget the whole thing) crossloaded to more than one factor. The goodness-of-fit of the three subscales were: $\chi^{2}(165)=295.103$, RMSEA 0.05 , CFI 0.95 , indicating a good fit of the model with the data. 


\section{Berlin Social Support Scales (BSSS).}

Social support is conceptualized in the literature as a construct consisting of many dimensions representing different kinds of support. The Berlin Social Support Scales (BSSS) is a multidimensional measure that was developed by Schwarzer and Schulz (2000) to assess cognitive and behavioral aspects of social support. The BSSS consists of six subscales: perceived, provided, received, need for support, support seeking, and protective buffering. In keeping with the social support that is operationalized in this study as perceived and received social supports, two of the six scales, perceived and received, were extrapolated for the purpose of answering the research question. One of the main advantages that the BSSS offers is its ability to measure the negative aspect of received social support, which studies have found to be linked to psychological distress. In addition, both subscales of perceived and received social support can be further categorized based on different functions each serves. For example, the perceived and received social support subscales encompass items that tap into emotional, informational, and instrumental supports, and the sum of these items produce scores for each type of support (Table 9). Furthermore, the received support also includes an item that measures the satisfaction with received support. The BSSS is a relatively new instrument for which studies examining the psychometric properties of the scale, i.e., EFA and/or CFA, are lacking. Although typically an EFA is performed to examine the latent factors underlying a set of measures, given the clear theoretical consideration outlined in the literature about social support, a decision was made to use CFA to examine whether the items conformed to the hypothesized scale of the structure (Hurley et al., 1997). 


\section{Table 9: Items of the Perceived Social Support Subscale}

\section{Item Description}

1 There are people who truly like me.

2 Whenever I am not feeling well, other people show me that they are fond of me.

3 Whenever I am sad, there are people who cheer me up.

4 There is always someone there for me when I need comforting.

$5 \quad$ I know some people upon whom I can always rely.

6 When I am worried, there is someone who helps me.

$7 \quad$ There are people who offer me help when I need it.

8 When everything becomes too much for me to handle, others are there to help me. 
As a first step for examining the structure of perceived and received social support scales, a CFA was performed on a two-factor model with two latent factors of perceived and received social support. Goodness-of-fit indices showed an acceptable fit: RMSEA 0.058 and CFI 0.974. We then proceeded to test the fit of each latent factor of social support. In other words, the CFA model of the perceived social support was specified as a single latent factor - perceived support - consisting of eight items. The goodness-of-fit indicated that the model was not a good fit with the data. The subscale of perceived social support consists of emotional and instrumental functions of support. Thus, the CFA model was re-specified as a two-factor model based on emotional and information support of perceived support with two factors consisting of four items each, i.e., items 1-4 for emotional factor and items 5-8 for instrumental factor. The goodness-of-fit indices were satisfactory with RMSEA 0.064 and CFI 995 (Table 10). Due to the complex nature of analysis of a model with a large number of latent variables, the decision was made to trim down the number of variables in the model. Evidence indicates that both emotional and information support have the most consistent link to outcomes such as depression and physical symptoms (Cohen, Mermelstein, Kamarck, \& Hoberman, 1985). Of the two functions of social support, many researchers believe that the strongest stress buffer is the emotional support that has the strongest association with quality of life (Helgeson, 2003). Thus, items (\#1 to 4) that assess emotional social support were selected to represent the latent variable of perceived social support (Figure 10). The emotional factor (items \#1-4) was chosen over the instrumental factor because the link to the psychological outcome was stronger and more consistent with emotional support across different studies (Helgeson, 2003; Uchino, 2004). 
Table 10: $\quad$ Berlin Social Support Scale: Perceived Social Support

\begin{tabular}{|c|c|c|c|c|c|c|}
\hline Model & Population & EFA/CFA & Number of & \multicolumn{2}{|c|}{ Original items numbers } & \multirow{2}{*}{ 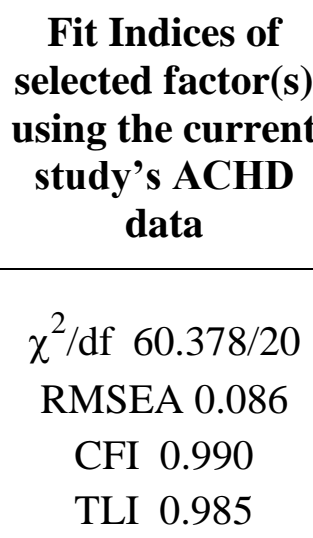 } \\
\hline $\begin{array}{l}\text { Schwarzer } \\
\& \text { Schulz } \\
(2000)\end{array}$ & $\begin{array}{l}\text { Originally designed } \\
\text { for individuals } \\
\text { coping with cancer }\end{array}$ & CFA & $\begin{array}{l}1 \\
\text { (entirely as } \\
\text { perceived } \\
\text { social support) }\end{array}$ & $\begin{array}{l}\text { F1 } \\
\text { (perceived } \\
\text { social } \\
\text { support) }\end{array}$ & $\begin{array}{l}1,2,3,4,5,6 \\
7,8\end{array}$ & \\
\hline \multirow{2}{*}{$\begin{array}{l}\text { Schwarzer } \\
\& \text { Schulz } \\
(2000)\end{array}$} & \multirow[t]{2}{*}{ Same as above } & \multirow[t]{2}{*}{$\begin{array}{l}\text { Same as } \\
\text { above }\end{array}$} & \multirow{2}{*}{$\begin{array}{l}2 \\
\text { (emotional \& } \\
\text { instrumental } \\
\text { dimensions) }\end{array}$} & $\begin{array}{l}\text { F1* } \\
\text { (Emotional) }\end{array}$ & $1,2,3,4$ & \multirow{2}{*}{$\begin{array}{c}\chi^{2} / \mathrm{df} 39.943 / 19 \\
\text { RMSEA } 0.064 \\
\text { CFI } 0.995 \\
\text { TLI } 0.992\end{array}$} \\
\hline & & & & $\begin{array}{l}\text { F2 } \\
\text { (Instrumental) }\end{array}$ & $5,6,7,8$ & \\
\hline
\end{tabular}

* F1 (shaded cell) =Emotional factor (subscale) selected to represent Perceived Social Support as a single dimension in the current study 
Figure 10. Proposed Measurement Model of Perceived Social Support to be Used in This Study With the ACHD Data

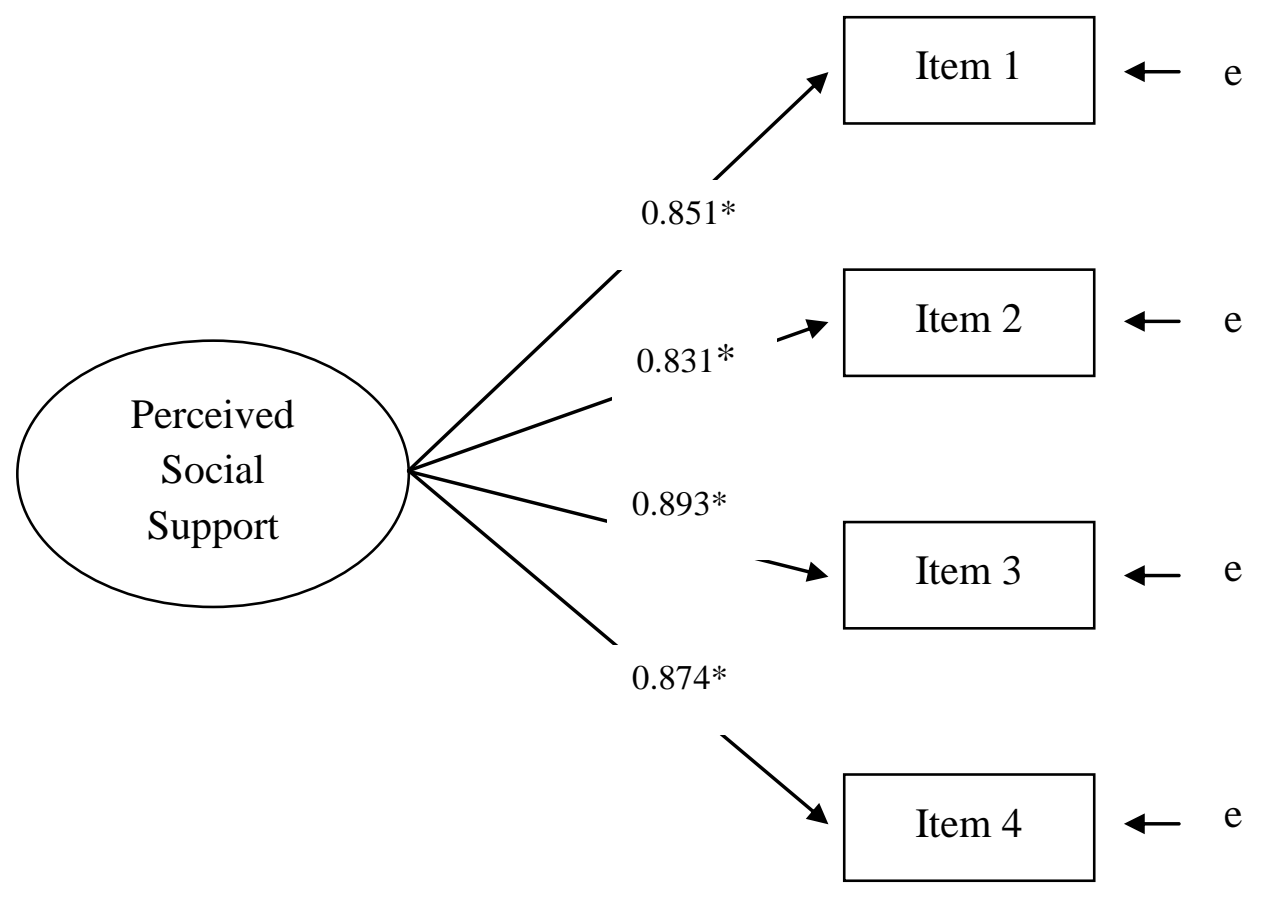

Fit indices: $\chi^{2}=2.371 d f=2$ RMSEA 0.026 CFI 1.000

* Standardized estimate, $p<0.000$ 
The validation of the received support measure was also carried out using CFA. Similar to the testing of perceived social support, the CFA model was first specified as a single factor consisting of the entire15 items in the scale. The goodness-of-fit indices indicated that the single factor model did not fit well with the data. One of the reasons for the lack of fit may have been attributed to the three negatively worded items: 4 (this person left me alone), 5(this person did not show much empathy for my situation), and 6(this person complained about me) (Table 11). At first, these three items were specified as a residual factor. However, the specification of a residual factor involving the three items led to a nonpositive definite matrix. Thus, the model was respecified by removing the residual factor and allowing the three error terms to correlate. The respecification rectified the issue and resulted in a positive definite matrix. However, the fit of the model was still poor. The examination of the analysis revealed a high residual for item number 14 (This person took care of things I could not manage on my own). When the model was respecified following the elimination of item number 14, the goodness-of-fit indices indicated an acceptable fit: RMSEA 0.077 and CFI 0.975 (Table 12). The final measurement model of received social support used for the testing of the structural model is presented in Figure 11. 


\section{Table 11: Items of the Received Social Support Subscale}

\begin{tabular}{ll}
\hline Item & \multicolumn{1}{c}{ Description } \\
\hline 1 & This person showed me that he/she loves and accepts me. \\
\hline 2 & This person was there when I needed him/her. \\
\hline 3 & This person comforted me when I was feeling bad. \\
\hline 4 & This person left me alone. \\
\hline 5 & This person did not show much empathy for my situation. \\
\hline 7 & This person complained about me. \\
\hline 8 & This person made me feel valued and important. \\
\hline 9 & This person expressed concern about my condition. \\
\hline 10 & This person assured me that I can rely completely on him/her. \\
\hline 11 & This person helped me find something positive in my situation. \\
\hline 12 & This person suggested activities that might distract me. \\
\hline 15 & This person encouraged me not to give up. \\
\hline
\end{tabular}


Table 12: $\quad$ Berlin Social Support Scale: Received Social Support

\begin{tabular}{|c|c|c|c|c|c|c|}
\hline Model & Factor Analysis & Modification & Reason for & \multicolumn{2}{|c|}{ Original items numbers } & $\begin{array}{c}\text { Fit Indices of } \\
\text { selected factor(s) }\end{array}$ \\
\hline \multirow{2}{*}{$\begin{array}{l}\text { Schwarzer } \\
\& \text { Schulz } \\
(2000)\end{array}$} & \multirow[t]{2}{*}{ CFA } & \multirow[t]{2}{*}{$\begin{array}{l}\text { Eliminate } \\
\text { item \#14 }\end{array}$} & \multirow[t]{2}{*}{ High residual } & $\begin{array}{l}\text { Factor } \\
\text { (Received) }\end{array}$ & $\begin{array}{l}1,2,3,4,5,6 \\
7,8,9,10,11, \\
12,13,15\end{array}$ & \multirow{2}{*}{$\begin{array}{l}\chi^{2} / \mathrm{df} 194.345 / 74 \\
\text { RMSEA } 0.077 \\
\text { CFI } 0.975\end{array}$} \\
\hline & & & & $\begin{array}{l}\text { Method } \\
\text { Factor } \\
\text { (negatively } \\
\text { worded items) }\end{array}$ & $4,5,6$ & \\
\hline
\end{tabular}


Figure 11. Proposed Measurement Model of Received Social Support to be Used in This Study With the ACHD Data

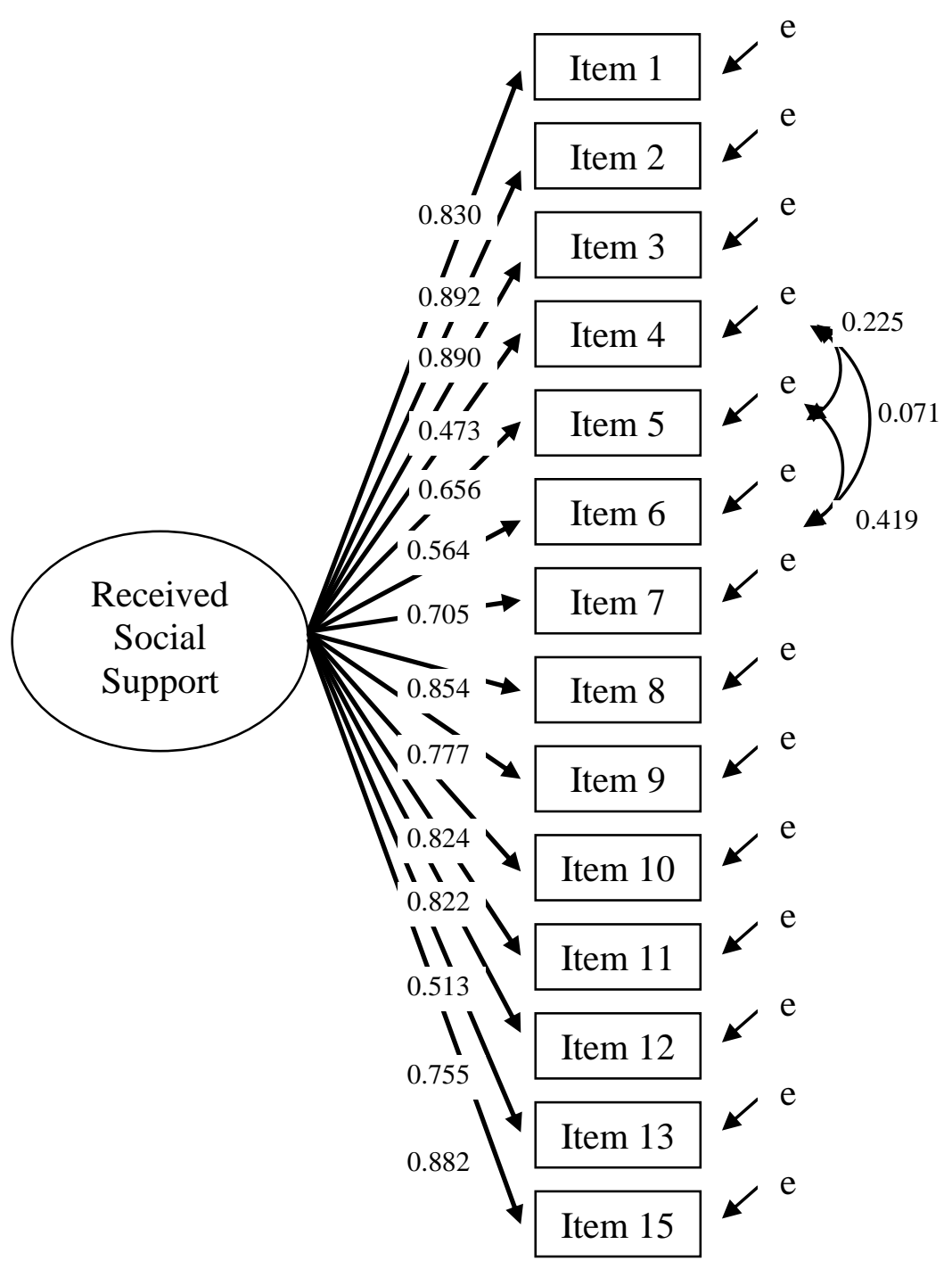

Fit indices: $\chi^{2}=194.345 d f=74 \quad$ RMSEA0.077 CFI 0.975

$\approx$ All estimates of received social support are standardized coefficients. 


\section{Examination of missing data.}

Prior to proceeding with the analysis of structural equation modeling, the data were examined for the frequency and pattern of missing data using the Mplus 6.0 software program. The WLSMV estimation method in Mplus uses the pairwise deletion as a default method to handle missing data. With the pairwise deletion, only the specific missing values, rather than subjects that have missing values on any of the variables under analysis, are excluded from the analysis. Thus, covariance estimates are calculated using all cases with complete observations on both variables (Brown, 2006); cases with missing data on the involved variables are removed from the associated analysis. The caveat of pairwise deletion is that covariance matrix may not be non-positive definite, and may cause a non-converging solution (Byrne, 2001). In addition the use of pairwise deletion can result in different sample sizes for the structural equation modeling. However, Muthén and Muthén (2006) claim that weighted lease squares is more robust with the pairwise deletion method.

The examination of missing data showed that of the 272 cases involved in the analysis, 249 cases had complete data on all study variables. A total percentage of missing values for all study variables was less than $5 \%$, which is an acceptable value (Tabachnick \& Fidell, 2007). The number of missing observations from all items of the instrument and from items involved in the analysis is presented in Table 13. The maximum number of missing variables per case was two. The proportion of covariance coverage of data ranged from 0.978 to 1.00 for all study variables. This means that all variables and pairs of variables consisted of $98 \%$ or more of the data present in the analysis. Thus, the percentage of 
missing data was $2 \%$ and this value was within the acceptable range of less than or equal to $5 \%$.

Table 13: Frequency of Missing Data in Study Variables

\begin{tabular}{|l|c|c|}
\hline \multicolumn{1}{|c|}{ Instrument } & \multicolumn{2}{|c|}{ Frequency (\%) } \\
\cline { 2 - 3 } & For total items & $\begin{array}{c}\text { For items used in } \\
\text { this study }\end{array}$ \\
\hline Beck Anxiety Inventory & $20(7)$ & $6(2)$ \\
\hline Beck Depression Inventory & $11(5)$ & $2(0.7)$ \\
\hline Ways of Coping Questionnaire & $49(18)$ & $13(4.7)$ \\
\hline Perceived Social Support & $4(1.4)$ & $0(0)$ \\
\hline Received Social Support & $15(5.5)$ & $13(4.7)$ \\
\hline
\end{tabular}

\section{Step 2: Testing of the Structural Models}

\section{Findings of structural equation modeling.}

Following the verification and confirmation of each of the measures, the relationships depicted in the structural models were tested. The following section presents the results of the structural equation modeling used to test the study's hypotheses. The analysis was conducted separately for the outcome variables of anxiety and depression. The findings pertaining to the outcome of anxiety are presented first, followed by the findings pertaining to the outcome of depression. All of the study variables were treated as ordered categorical (ordinal) variables. The Mplus version 6.0 software program was used with the 
estimating method of mean- and variance-adjusted weighted least squares (WLSMV). The assessment of the model fit was established using the following criteria: RMSEA $\leq .08, \mathrm{CFI}$ $>0.95$, and TLI $\geq 0.95$. For a selected number of models, a minor revision was necessary. The identification of the source of misfits in the models was guided by the inspected values of residuals, MI, R-squared, and conceptual consistency between the observed variable and the construct.

Bivariate statistics of the study variables are presented in Table 14. Anxiety and depression demonstrated a high correlation with each other $(\mathrm{r}=0.83)$, indicating that both variables are measuring similar constructs. For the outcome variables of both anxiety and depression, perceived social support showed a high correlation with received social support $(\mathrm{r}=0.73)$. Anxiety was moderately and negatively correlated with perceived social support $(\mathrm{r}=-0.41)$ whereas depression was moderately and negatively correlated with both perceived and received social support ( $\mathrm{r}=-0.59$ and -0.47 , respectively). The coping strategy of wishful-thinking showed a moderate correlation with both anxiety and depression $(\mathrm{r}=0.55$ and 0.63 , respectively). The bivariate correlations between each of perceived and received social support and problem-solving coping strategy were also moderate $(\mathrm{r}=0.35$ and 0.31 , respectively). 
Table 14: $\quad$ Estimated Correlation Matrix for the Latent Variables

\begin{tabular}{|c|c|c|c|c|c|c|c|}
\hline & $\begin{array}{l}\text { Perceived } \\
\text { Social } \\
\text { Support }\end{array}$ & $\begin{array}{c}\text { Received } \\
\text { Social } \\
\text { Support }\end{array}$ & $\begin{array}{c}\text { Problem- } \\
\text { Solving }\end{array}$ & $\begin{array}{l}\text { Wishful } \\
\text { Thinking }\end{array}$ & $\begin{array}{c}\text { Denial/ } \\
\text { Avoidance }\end{array}$ & Anxiety & Depression \\
\hline $\begin{array}{l}\text { Perceived } \\
\text { Social Support }\end{array}$ & 1 & & & & & & \\
\hline $\begin{array}{l}\text { Received } \\
\text { Social Support }\end{array}$ & 0.725 & 1 & & & & & \\
\hline $\begin{array}{l}\text { Problem- } \\
\text { Solving }\end{array}$ & 0.350 & 0.313 & 1 & & & & \\
\hline Wishful Thinking & -0.211 & -0.165 & 0.114 & 1 & & & \\
\hline Denial/Avoidance & -0.035 & -0.087 & 0.150 & 0.538 & 1 & & \\
\hline Anxiety & -0.406 & -0.289 & 0.000 & 0.551 & 0.218 & 1 & \\
\hline Depression & -0.588 & -0.465 & -0.131 & 0.626 & 0.250 & 0.827 & 1 \\
\hline
\end{tabular}




\section{Anxiety (Model 1).}

The hypotheses of the study were perceived and received social support are significantly associated with psychological distress (anxiety) either directly, or indirectly through different coping strategies of individuals living with CHD (Figure 12). The hypotheses were partially supported. The results of the analysis showed a significant relationship between social support and anxiety. The only significant relationship was the direct relationship between perceived social support and anxiety $(p=0.018)$. In other words, as the perception of social support decreased, the level of anxiety increased. Perceived social support was also indirectly related to anxiety separately through wishful-thinking and problem-solving coping strategies but these relationships were only partially mediated (Figure 13). In contrast, received social support failed to demonstrate any significant relationship with neither different coping strategies nor anxiety. Findings of the parameter estimate, standard error, significance test and fit indices of the Model 1are presented in Table 15 .

Given the lack of significant relationship between received social support and other study variables (i.e., coping strategies and anxiety) delineated in the model, the decision was made to repeat the analysis of the SEM while constraining the effect of received social support at 0 (Model 2). Constraining the effects of received social support produced a slight alteration in the outcome: in addition to the significant direct relationship between perceived social support and anxiety, the indirect effect between perceived social support and anxiety mediated by wishful-thinking coping strategies became significant. In contrast, the partial mediation effect of problem-solving coping strategies between perceived social support 
Figure 12. Model 1: The Effects of Social Support (Perceived \& Received) and Coping Strategies (ProblemSolving, Wishful-Thinking and Denial/Avoidance) on Anxiety

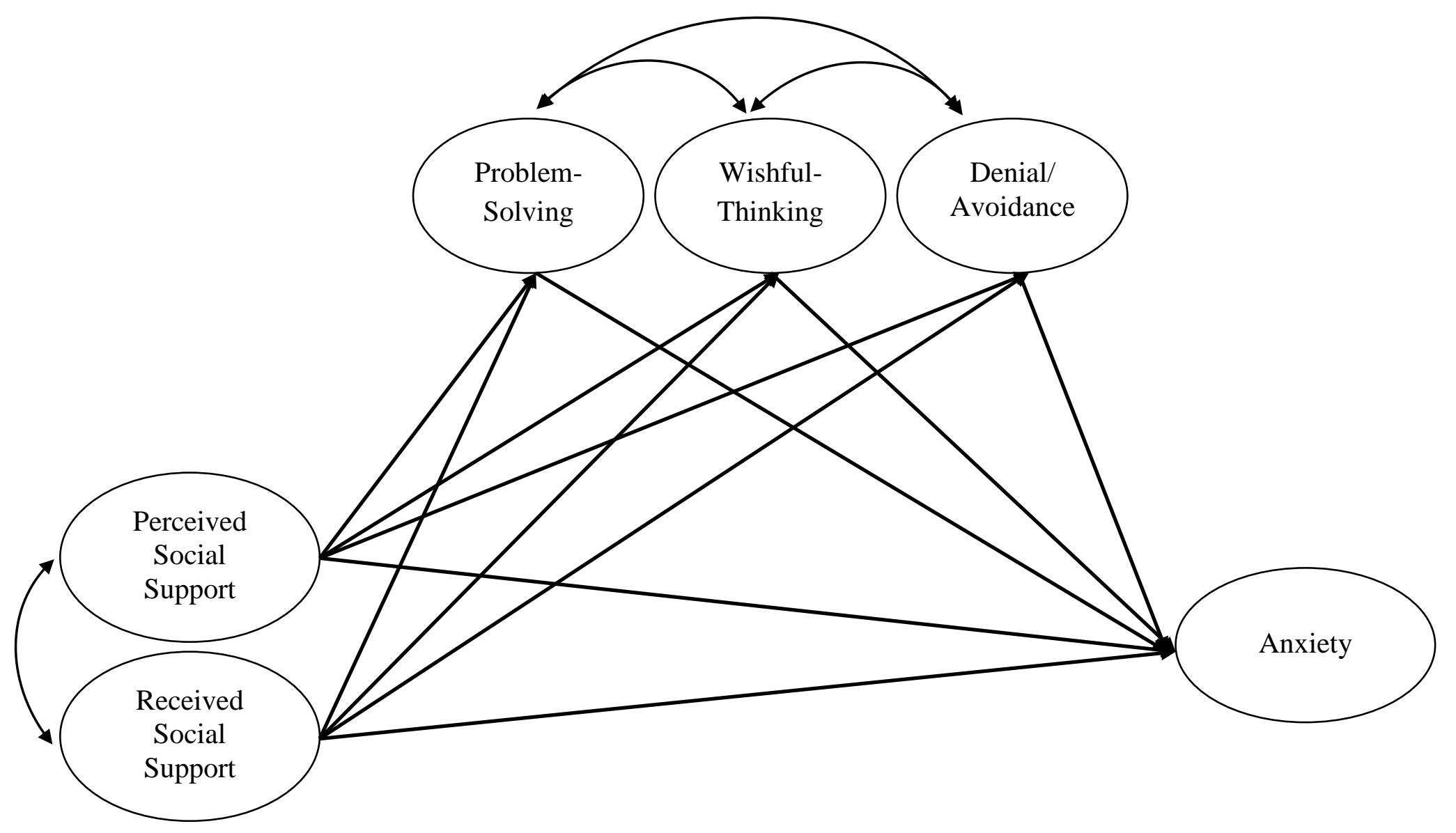


Table 15: Model 1: The Effects of Types of Social Support and Types of Coping Strategies on Anxiety

\begin{tabular}{|c|c|c|c|c|c|}
\hline Path of Latent Variable & Estimate B & Estimate $\beta$ & $\begin{array}{l}\text { Standard } \\
\text { Error }\end{array}$ & P - Value & Fit Indices \\
\hline Problem-solving Coping $\rightarrow$ Anxiety & 0.092 & 0.067 & 0.071 & 0.348 & \multirow{11}{*}{$\begin{array}{lr}\chi 2(d f) & 1149.851(882) \\
\text { RMSEA } & 0.033 \\
95 \% \text { CI } & 0.028-0.039 \\
\text { CFI } & 0.966\end{array}$} \\
\hline Wishful-thinking Coping $\rightarrow$ Anxiety & 0.522 & 0.526 & 0.087 & 0.000 & \\
\hline Denial/Avoidance Coping $\rightarrow$ Anxiety & -0.124 & -0.089 & 0.104 & 0.393 & \\
\hline Perceived Social Support $\rightarrow$ Anxiety & -0.259 & -0.300 & 0.126 & 0.018 & \\
\hline Received Social Support $\rightarrow$ Anxiety & -0.028 & -0.032 & 0.109 & 0.766 & \\
\hline $\begin{array}{l}\text { Perceived Social Support } \rightarrow \text { Problem- } \\
\text { solving Coping }\end{array}$ & 0.165 & 0.265 & 0.127 & 0.037 & \\
\hline $\begin{array}{l}\text { Perceived Social Support } \rightarrow \text { Wishful- } \\
\text { thinking Coping }\end{array}$ & -0.152 & -0.175 & 0.127 & 0.167 & \\
\hline $\begin{array}{l}\text { Perceived Social Support } \rightarrow \\
\text { Denial/Avoidance Coping }\end{array}$ & 0.050 & 0.082 & 0.140 & 0.561 & \\
\hline $\begin{array}{l}\text { Received Social Support } \rightarrow \text { Problem-solving } \\
\text { Coping }\end{array}$ & 0.075 & 0.118 & 0.123 & 0.335 & \\
\hline $\begin{array}{l}\text { Received Social Support } \rightarrow \text { Wishful-thinking } \\
\text { Coping }\end{array}$ & -0.036 & -0.041 & 0.118 & 0.731 & \\
\hline $\begin{array}{l}\text { Received Social Support } \rightarrow \\
\text { Denial/Avoidance Coping }\end{array}$ & -0.103 & -0.164 & 0.137 & 0.233 & \\
\hline
\end{tabular}


Figure 13. Significant Paths for Model 1: The Effects of Social Support (Perceived \& Received) and Coping Strategies (Problem -Solving, Wishful-Thinking and Denial/Avoidance) on Anxiety

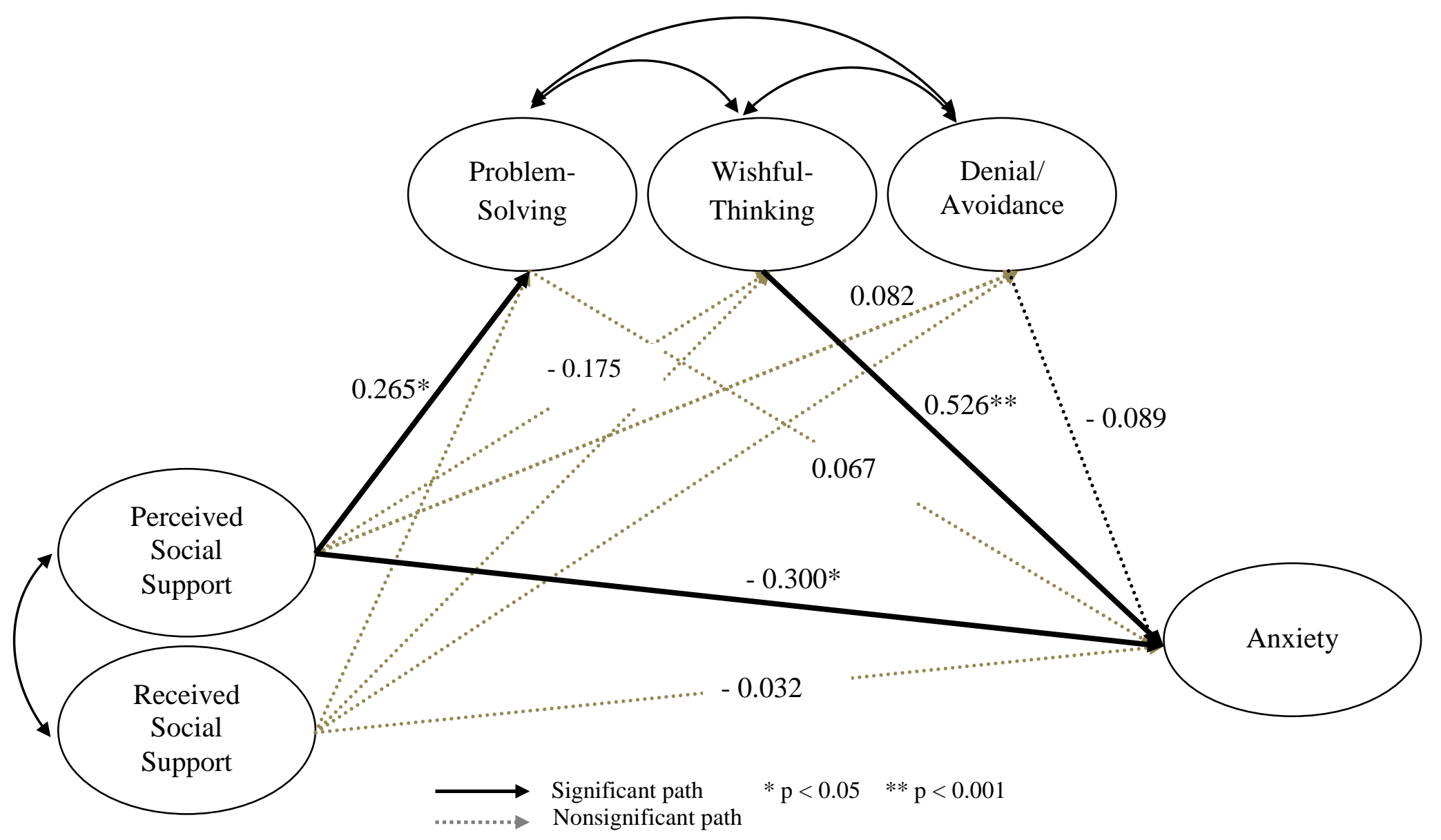


and anxiety completely disappeared and became nonsignificant. Thus, the person with decreased perception of social support used more wishful-thinking coping strategies, which in turn, increased one's level of anxiety (Figure 14). The parameter estimate, standard error, significance test and fit indices of the revised model with the constrained effect of received social support are presented in Table 16. The chi-square test for testing the difference in fit was then used to determine whether there was significant difference between the original model in which the effects of received social support are freely estimated and the revised model in which the effects of received model are constrained. The test was insignificant $\left(\chi^{2}(d f)=2.848(4), p=0.58\right)$ and therefore, it was concluded that the inclusion of received social support in the model would not significantly increase the fit of the model. The estimates, standard error and significance values for direct and indirect effects of the revised model are presented in Table 17.

However, one other aspect of the model needed to be tested before removing the variable of received social support from the model. The results of bivariate correlation matrix revealed a high correlation between perceived and received social support. This implies the possibility that perceived social support is mediating the relationship between received social support and psychological distress (Figure 15). Thus, an additional analysis was performed to examine the possible mediating effect of perceived social support on the relationship between received social support and anxiety. In order to test this relationship, a direct path was estimated from received support to perceived support, to represent the influence of received social support on perceived social support, which in turn, influences 
Table 16: Model 2: The Effects of Perceived Social Support and Types of Coping Strategies on Anxiety With the Constraint $^{\S}$ of Received Social Support

\begin{tabular}{|c|c|c|c|c|c|}
\hline Path of Latent Variable & Estimate B & Estimate $\beta$ & $\begin{array}{l}\text { Standard } \\
\text { Error }\end{array}$ & P - Value & Fit Indices \\
\hline Problem-solving Coping $\rightarrow$ Anxiety & 0.125 & 0.090 & 0.076 & 0.237 & \multirow{7}{*}{$\begin{array}{lr}\chi 2(d f) & 1134.762(886) \\
\text { RMSEA } & 0.032 \\
95 \% \text { CI } & 0.026-0.038 \\
\text { CFI } & 0.969\end{array}$} \\
\hline Wishful-thinking Coping $\rightarrow$ Anxiety & 0.524 & 0.528 & 0.088 & 0.000 & \\
\hline Denial/Avoidance Coping $\rightarrow$ Anxiety & -0.161 & -0.113 & 0.104 & 0.276 & \\
\hline Perceived Social Support $\rightarrow$ Anxiety & -0.300 & -0.344 & 0.084 & 0.000 & \\
\hline $\begin{array}{l}\text { Perceived Social Support } \rightarrow \text { Problem-solving } \\
\text { Coping }\end{array}$ & 0.248 & 0.393 & 0.065 & 0.000 & \\
\hline $\begin{array}{l}\text { Perceived Social Support } \rightarrow \text { Wishful-thinking } \\
\text { Coping }\end{array}$ & -0.192 & -0.219 & 0.068 & 0.001 & \\
\hline $\begin{array}{l}\text { Perceived Social Support } \rightarrow \text { Denial/Avoidance } \\
\text { Coping }\end{array}$ & -0.062 & -0.100 & 0.093 & 0.281 & \\
\hline
\end{tabular}

$\mathrm{B}=$ unstandardized parameter estimates, $\quad \beta=$ standardized parameter estimates

$\S$ The effects of received social support on coping strategies and anxiety were constrained at 0 . 
Table 17: Chi-Square Test of the Difference in Fit Between the Model 1 (Effects of Received Social Support are Freely Estimated) and the Model 2 (Effects of Received Social Support are Constrained)

\begin{tabular}{|c|c|c|c|c|c|c|}
\hline Path of Latent Variable & Estimate B & Estimate $\beta$ & $\begin{array}{l}\text { Standard } \\
\text { Error }\end{array}$ & P - Value & $\begin{array}{c}\text { Mediation } \\
\text { (\% of the } \\
\text { Total Effect) }\end{array}$ & $\begin{array}{c}\chi 2 \text { Test }^{\S} \text { for } \\
\text { Difference Testing }\end{array}$ \\
\hline Total Direct & -0.360 & -0.413 & 0.070 & 0.000 & & \multirow{7}{*}{$\begin{array}{lr}\chi 2(d f) & 2.848(4) \\
\text { P-Value } & 0.584\end{array}$} \\
\hline Direct & & & & & & \\
\hline Perceived Social Support $\rightarrow$ Anxiety & -0.300 & -0.344 & 0.084 & 0.000 & & \\
\hline \multicolumn{6}{|l|}{ Specific Indirect } & \\
\hline $\begin{array}{l}\text { Perceived Social Support } \rightarrow \text { Problem- } \\
\text { solving Coping } \rightarrow \text { Anxiety }\end{array}$ & 0.031 & 0.035 & 0.031 & 0.257 & 8.5 & \\
\hline $\begin{array}{l}\text { Perceived Social Support } \rightarrow \text { Wishful- } \\
\text { thinking Coping } \rightarrow \text { Anxiety }\end{array}$ & -0.101 & -0.116 & 0.039 & 0.003 & 28.1 & \\
\hline $\begin{array}{l}\text { Perceived Social Support } \rightarrow \\
\text { Denial/Avoidance Coping } \rightarrow \text { Anxiety }\end{array}$ & 0.010 & 0.011 & 0.016 & 0.489 & 2.7 & \\
\hline
\end{tabular}


Figure 14. Significant Paths for Model 2: The Effects of Social Support (Perceived \& Received) and Coping Strategies (Problem-Solving, Wishful-Thinking and Denial/Avoidance) on Anxiety with the Effects of Received Social Support Constrained

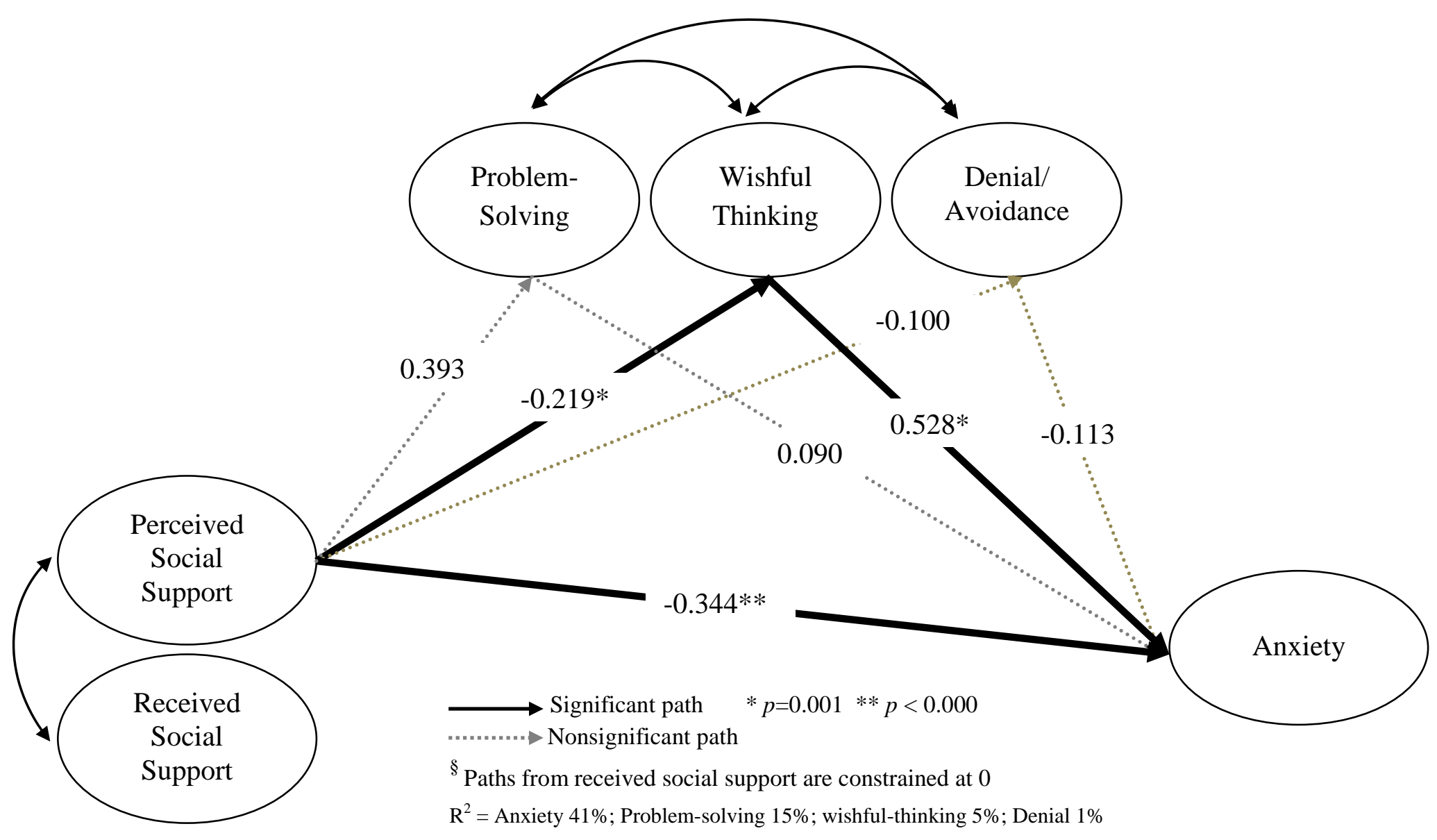


anxiety (Model 3). The result suggests that received social support influences the level of anxiety indirectly through perceived social support (Table 18). It is important to note, however, that the models depicted in Figure 14 and Figure 16 are the same model represented in alternative specifications. Thus, the statistical determination of whether the relationship between perceived and received social support is best represented as a correlation or as a directional association is not possible.

In summary, the final model reflecting the result of the study consists of following significant relationships: 1) perceived social support directly influences the level of anxiety, 2) perceived social support influences the level of anxiety through wishful-thinking coping strategies, and 3) received social support influences the level of anxiety indirectly through perceived social support (Figure 16). 


\section{Figure 15. Model 3: The Effect of Received Social Support on Anxiety Mediated by Perceived Social Support}

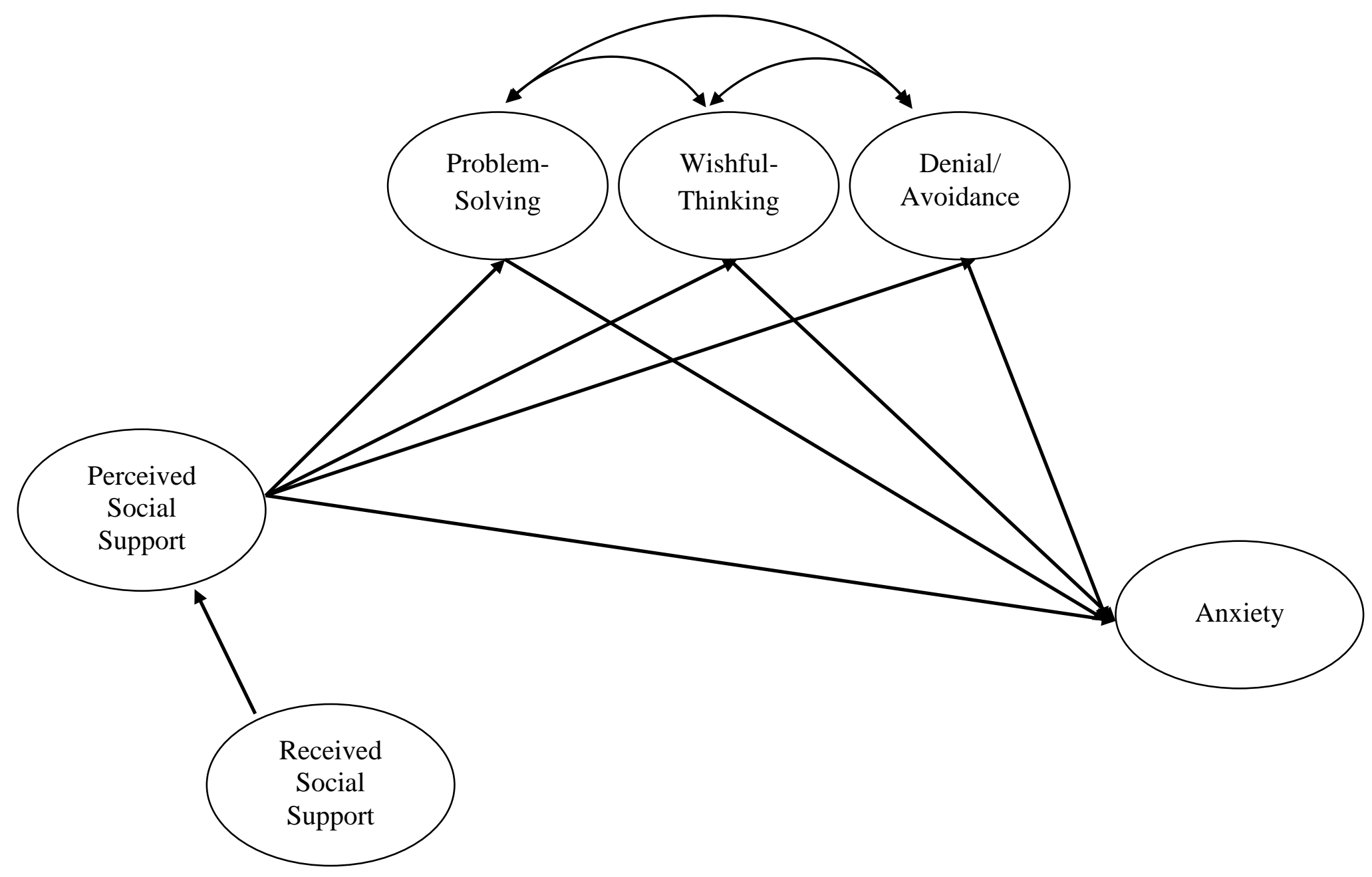


Table 18: Model 3: The Effect of Received Social Support on Anxiety Mediated by Perceived Social Support

\begin{tabular}{|c|c|c|c|c|c|}
\hline Path of Latent Variable & Estimate B & Estimate $\beta$ & $\begin{array}{l}\text { Standard } \\
\text { Error }\end{array}$ & P - Value & Fit Indices \\
\hline Problem- solving Coping $\rightarrow$ Anxiety & 0.125 & 0.090 & 0.076 & 0.238 & \multirow{8}{*}{$\begin{array}{lr}\chi 2(d f) & 1134.762(886) \\
\text { RMSEA } & 0.032 \\
95 \% \text { CI } & 0.026-0.038 \\
\text { CFI } & 0.969\end{array}$} \\
\hline Wishful-thinking Coping $\rightarrow$ Anxiety & 0.524 & 0.528 & 0.088 & 0.000 & \\
\hline Denial/Avoidance Coping $\rightarrow$ Anxiety & -0.161 & -0.113 & 0.104 & 0.276 & \\
\hline Perceived Social Support $\rightarrow$ Anxiety & -0.300 & -0.344 & 0.084 & 0.000 & \\
\hline $\begin{array}{l}\text { Perceived Social Support } \rightarrow \text { Problem- solving } \\
\text { Coping }\end{array}$ & 0.248 & 0.393 & 0.065 & 0.000 & \\
\hline $\begin{array}{l}\text { Perceived Social Support } \rightarrow \text { Wishful-thinking } \\
\text { Coping }\end{array}$ & -0.192 & -0.219 & 0.068 & 0.001 & \\
\hline $\begin{array}{l}\text { Perceived Social Support } \rightarrow \text { Denial/Avoidance } \\
\text { Coping }\end{array}$ & -0.062 & -0.100 & 0.093 & 0.281 & \\
\hline $\begin{array}{l}\text { Received Social Support } \rightarrow \text { Perceived Social } \\
\text { Support }\end{array}$ & 0.741 & 0.737 & 0.043 & 0.000 & \\
\hline
\end{tabular}

$\mathrm{B}=$ unstandardized parameter estimates, $\quad \beta=$ standardized parameter estimates

$\S$ The effects of received social support on coping strategies and anxiety were constrained at 0 . 
Figure 16. Significant Paths for Model 3: The Indirect Effects of Perceived Social Support on the Relationship Between Received Social Support ${ }^{\S}$ and Anxiety

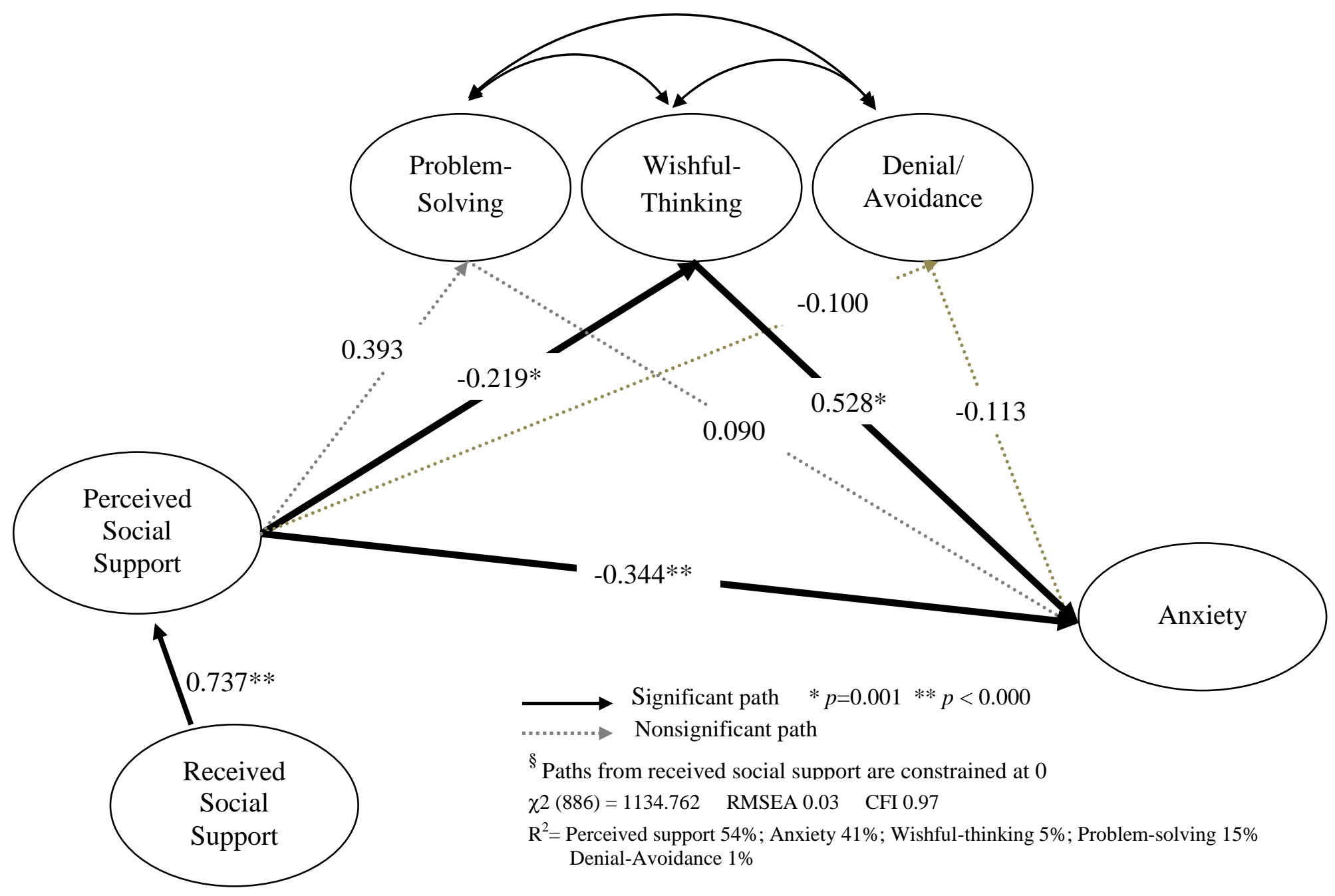




\section{Depression (Model 4).}

The hypotheses of the study were that perceived and received social support are significantly associated with psychological distress (depression), either directly or indirectly through different coping strategies of individuals living with CHD (Figure 17). Results of the analysis pertaining to depression were similar to the analysis results for anxiety. The only significant relationship was the direct relationship between perceived social support and depression $(p<0.001)$. In other words, as the perception of social support decreased, the level of depression increased. The relationship between perceived social support and depression was only partially mediated by each of problem-solving and wishful-thinking coping strategies. Consistent with its effect on anxiety, received social support failed to demonstrate any significant relationship with different coping strategies and depression (Figure 18). Findings of the parameter estimate, standard error, significance test and fit indices of the Model 4 are presented in Table 19.

Given the lack of significant relationships between received social support and any of the study variables (i.e., coping strategies and depression), the decision was made to repeat the analysis of the SEM while constraining the effects of received social support at 0 (Model 5). The difference in outcome was observed when the effects of received support were constrained. The significant direct relationship between perceived social support and depression remained unchanged. However, the partial mediation effect of wishful-thinking coping strategies on the relationship between perceived social support and depression became the full mediation effect. On the other hand, the partial mediation effect 
Figure 17. Model 4: The Effects of Social Support (Perceived \& Received) and Coping Strategies (Problem-Solving, Wishful-Thinking and Denial/Avoidance) on Depression

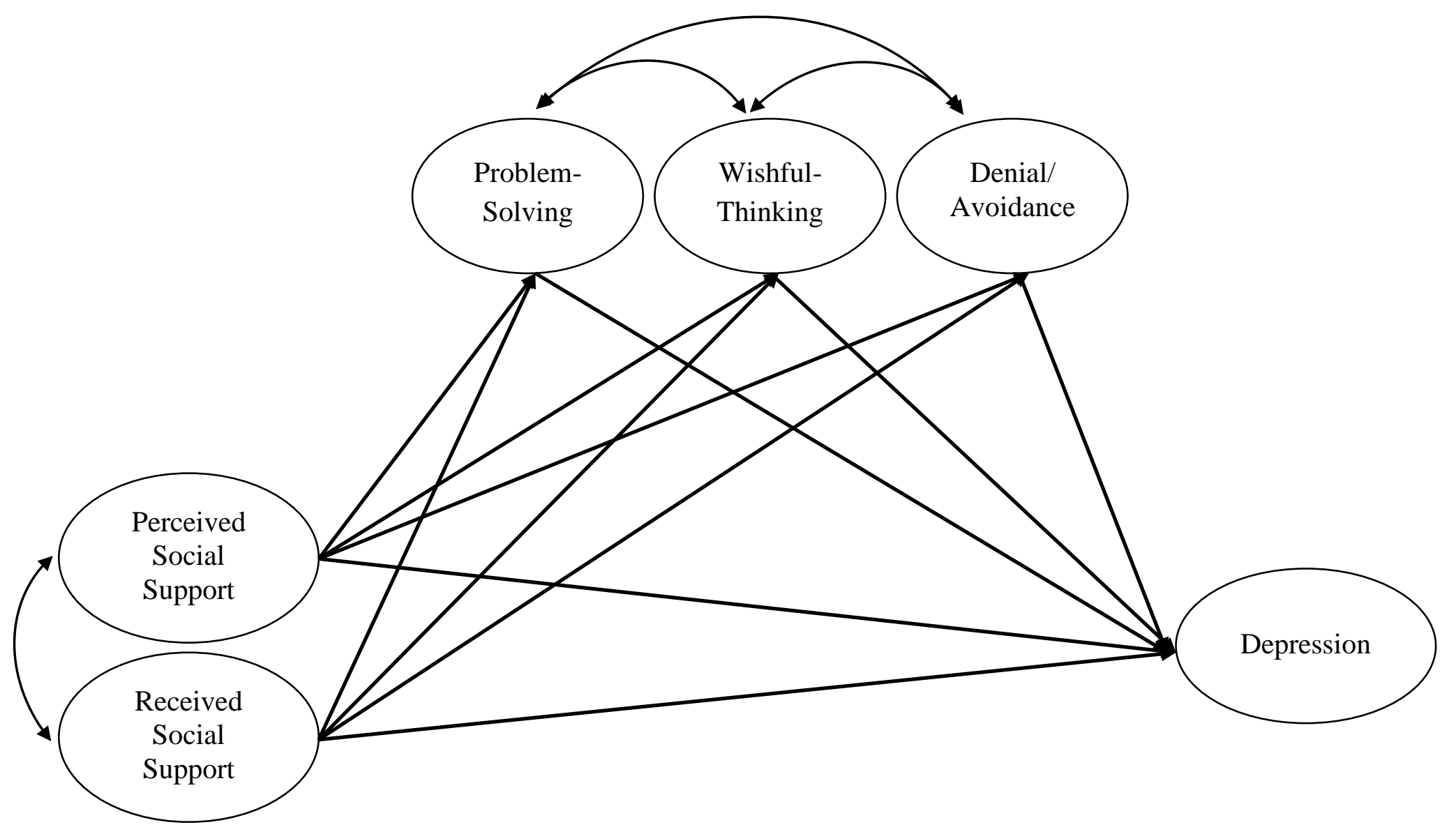


Table 19: $\quad$ Model 4: The Effects of Types of Social Support and Types of Coping Strategies on Depression

\begin{tabular}{|c|c|c|c|c|c|}
\hline Path of Latent Variable & Estimate B & Estimate $\beta$ & Standard Error & P - Value & Fit Indices \\
\hline Problem-solving Coping $\rightarrow$ Depression & -0.025 & -0.015 & 0.064 & 0.814 & \multirow{11}{*}{$\begin{array}{lr}\chi 2(d f) & 1537.519(1254) \\
\text { RMSEA } & 0.029 \\
95 \% \text { CI } & 0.023-0.034 \\
\text { CFI } & 0.967\end{array}$} \\
\hline Wishful-thinking Coping $\rightarrow$ Depression & 0.694 & 0.572 & 0.078 & 0.000 & \\
\hline Denial/Avoidance Coping $\rightarrow$ Depression & -0.136 & -0.081 & 0.090 & 0.367 & \\
\hline Perceived Social Support $\rightarrow$ Depression & -0.396 & -0.389 & 0.098 & 0.000 & \\
\hline Received Social Support $\rightarrow$ Depression & -0.114 & -0.107 & 0.084 & 0.202 & \\
\hline $\begin{array}{l}\text { Perceived Social Support } \rightarrow \text { Problem-solving } \\
\text { Coping }\end{array}$ & 0.163 & 0.266 & 0.127 & 0.036 & \\
\hline $\begin{array}{l}\text { Perceived Social Support } \rightarrow \text { Wishful-thinking } \\
\text { Coping }\end{array}$ & -0.150 & -0.179 & 0.126 & 0.157 & \\
\hline $\begin{array}{l}\text { Perceived Social Support } \rightarrow \\
\text { Denial/Avoidance Coping }\end{array}$ & 0.052 & 0.086 & 0.141 & 0.540 & \\
\hline $\begin{array}{l}\text { Received Social Support } \rightarrow \text { Problem-solving } \\
\text { Coping }\end{array}$ & 0.075 & 0.118 & 0.123 & 0.339 & \\
\hline $\begin{array}{l}\text { Received Social Support } \rightarrow \text { Wishful-thinking } \\
\text { Coping }\end{array}$ & -0.038 & -0.043 & 0.118 & 0.714 & \\
\hline $\begin{array}{l}\text { Received Social Support } \rightarrow \text { Denial/Avoidance } \\
\text { Coping }\end{array}$ & -0.106 & -0.168 & 0.138 & 0.225 & \\
\hline
\end{tabular}

$\mathrm{B}=$ unstandardized parameter estimates,$\quad \beta=$ standardized parameter estimates 
Figure 18. Significant Paths for Model 4: The Effects of Social Support (Perceived \& Received) and Coping Strategies (Problem-Solving, Wishful-Thinking and Denial/Avoidance) on Depression

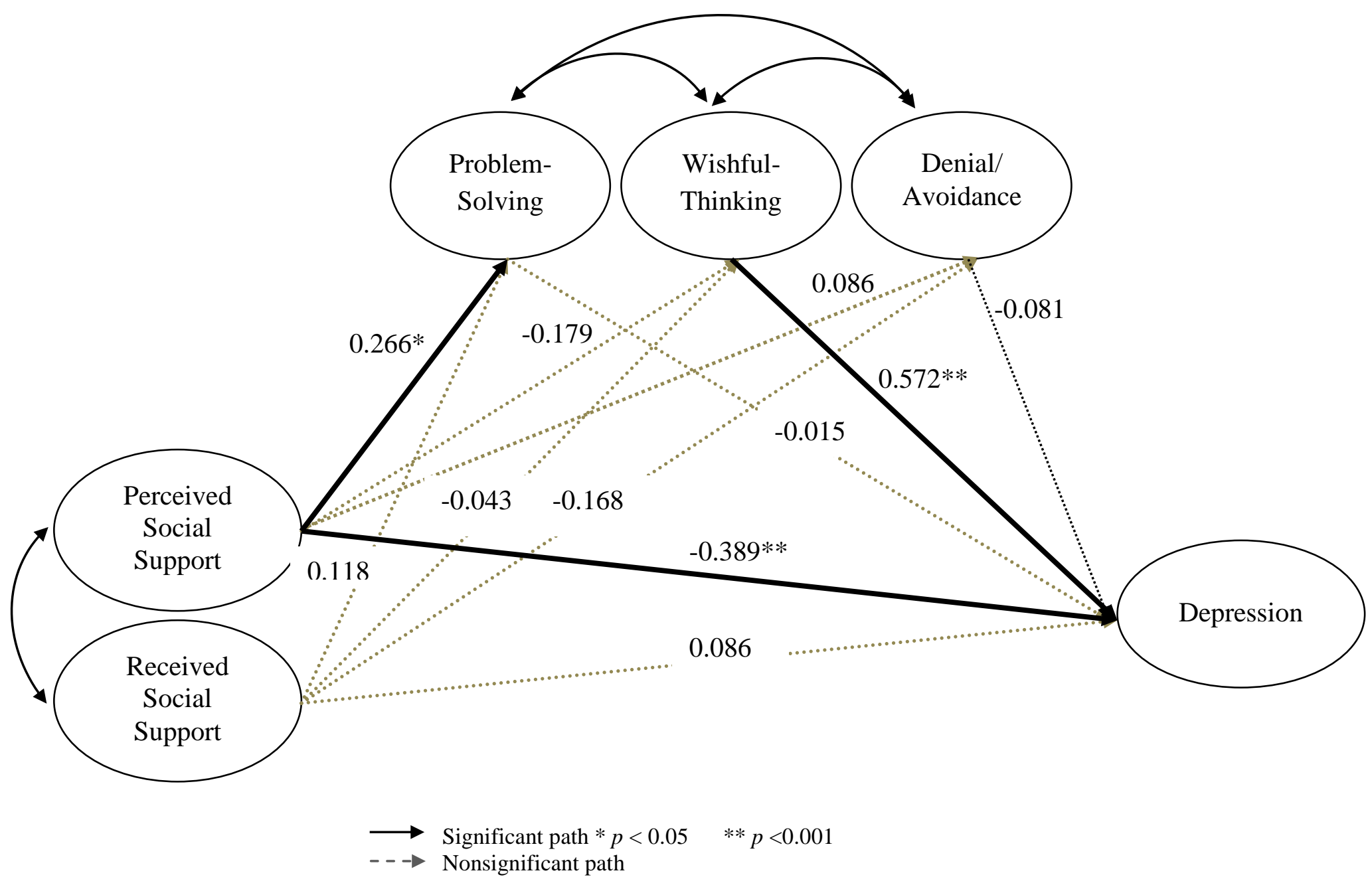


of problem-solving coping strategies remained unchanged (Table 20). The chi-square test for testing the difference in fit was then used to determine whether there was a significant difference between the original model in which the effects of received social support are freely estimated and the revised model in which the effects of received model are constrained (Figure 19). The test was nonsignificant $\left(\chi^{2}(d f)=3.712(4), p=0.45\right)$ and therefore, it was concluded that the inclusion of received social support in the model would not significantly increase the fit of the model. The estimates, standard error and significance values for direct and indirect effects of the revised model are presented in Table 21.

However, one other aspect of the model needed to be tested before removing the variable of received social support completely from the model of depression. The results of bivariate correlation matrix revealed a high correlation between perceived and received social support. This implies the possibility that perceived social support is mediating the relationship between received social support and psychological distress (Figure 20). Thus, an additional analysis was performed to examine the possible mediating effect of perceived social support on the relationship between received social support and depression. Same as for the analysis of anxiety, a direct path was estimated from received support to perceived support, to represent the influence of received social support on perceived social support, which in turn, influenced depression (Model 6). The result confirmed the suspicion that received social support influences the level of anxiety indirectly through perceived social support (Table 22). Therefore, the final model of depression reflecting the result of the study consists of following significant relationships: 1) perceived social support directly influences the level of depression, 2) perceived social support influences the level of 
Table 20: Model 5: The Effects of Perceived Social Support and Types of Coping Strategies on Depression With the Effects of Received Social Support Constrained ${ }^{\S}$

\begin{tabular}{|l|c|c|c|c|c|}
\hline \multicolumn{1}{|c|}{ Path of Latent Variable } & Estimate B & Estimate $\boldsymbol{\beta}$ & $\begin{array}{c}\text { Standard } \\
\text { Error }\end{array}$ & P - Value & Fit Indices \\
\hline Problem-solving Coping $\rightarrow$ Depression & 0.036 & 0.022 & 0.069 & 0.750 \\
\hline Wishful-thinking Coping $\rightarrow$ Depression & 0.691 & 0.570 & 0.079 & $\mathbf{0 . 0 0 0}$ & $\begin{array}{l}\chi 2(d f) 1531.125(1258) \\
\text { RMSEA } \\
0.028\end{array}$ \\
\hline Denial/Avoidance Coping $\rightarrow$ Depression & -0.191 & -0.112 & 0.091 & 0.222 \\
\hline CFI \\
\hline $\begin{array}{l}\text { Perceived Social Support } \rightarrow \text { Depression } \\
\text { Perceived Social Support } \rightarrow \text { Problem-solving } \\
\text { Coping }\end{array}$ & -0.538 & -0.517 & $0.023-0.033$ \\
\hline $\begin{array}{l}\text { Perceived Social Support } \rightarrow \text { Wishful-thinking } \\
\text { Coping }\end{array}$ & -0.191 & -0.223 & 0.068 \\
\hline $\begin{array}{l}\text { Perceived Social Support } \rightarrow \text { Denial/Avoidance } \\
\text { Coping }\end{array}$ & -0.061 & -0.100 & 0.067 & $\mathbf{0 . 0 0 0}$ \\
\hline
\end{tabular}

$\mathrm{B}=$ unstandardized parameter estimates, $\quad \beta=$ standardized parameter estimates

$\S$ The effects of received social support on coping strategies and anxiety were constrained at 0 . 
Table 21: Chi-Square Test of the Difference in Fit Between the Model 4 (Effects Of Received Social Support are Freely Estimated) and the Model 5 (Effects of Received Social Support are Constrained)

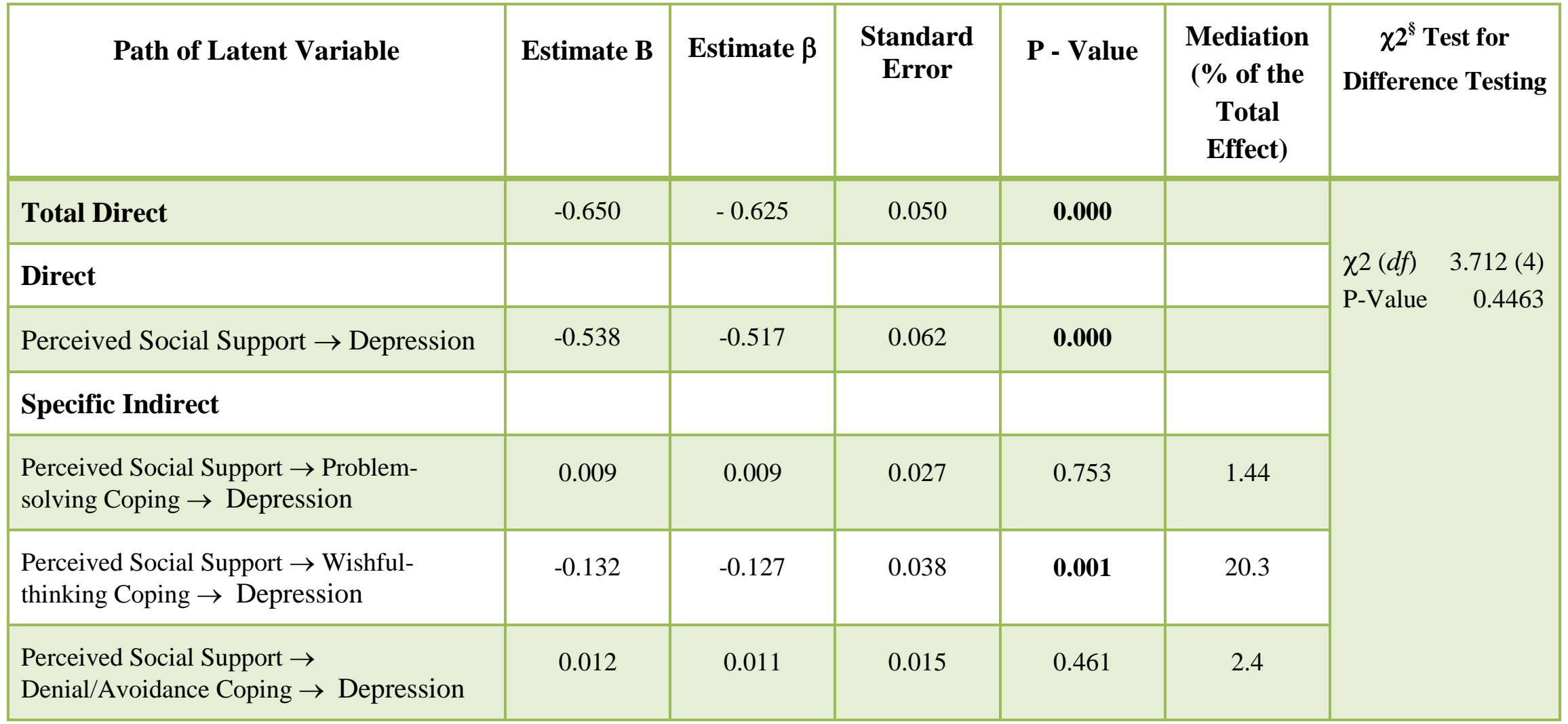

$\mathrm{B}=$ unstandardized parameter estimates, $\quad \beta=$ standardized parameter estimates

$\S$ Chi-square difference test refers to the test of difference in fit between the model with freely estimated received social support and the model with constrained received social support (paths from received social support are constrained at 0 ). 
Figure 19. Significant Paths for Model 5: The Effects of Social Support (Perceived \& Received) and Coping Strategies (Problem-Solving, Wishful-Thinking and Denial/Avoidance) on Depression With the Effects of Received Social Support Constrained

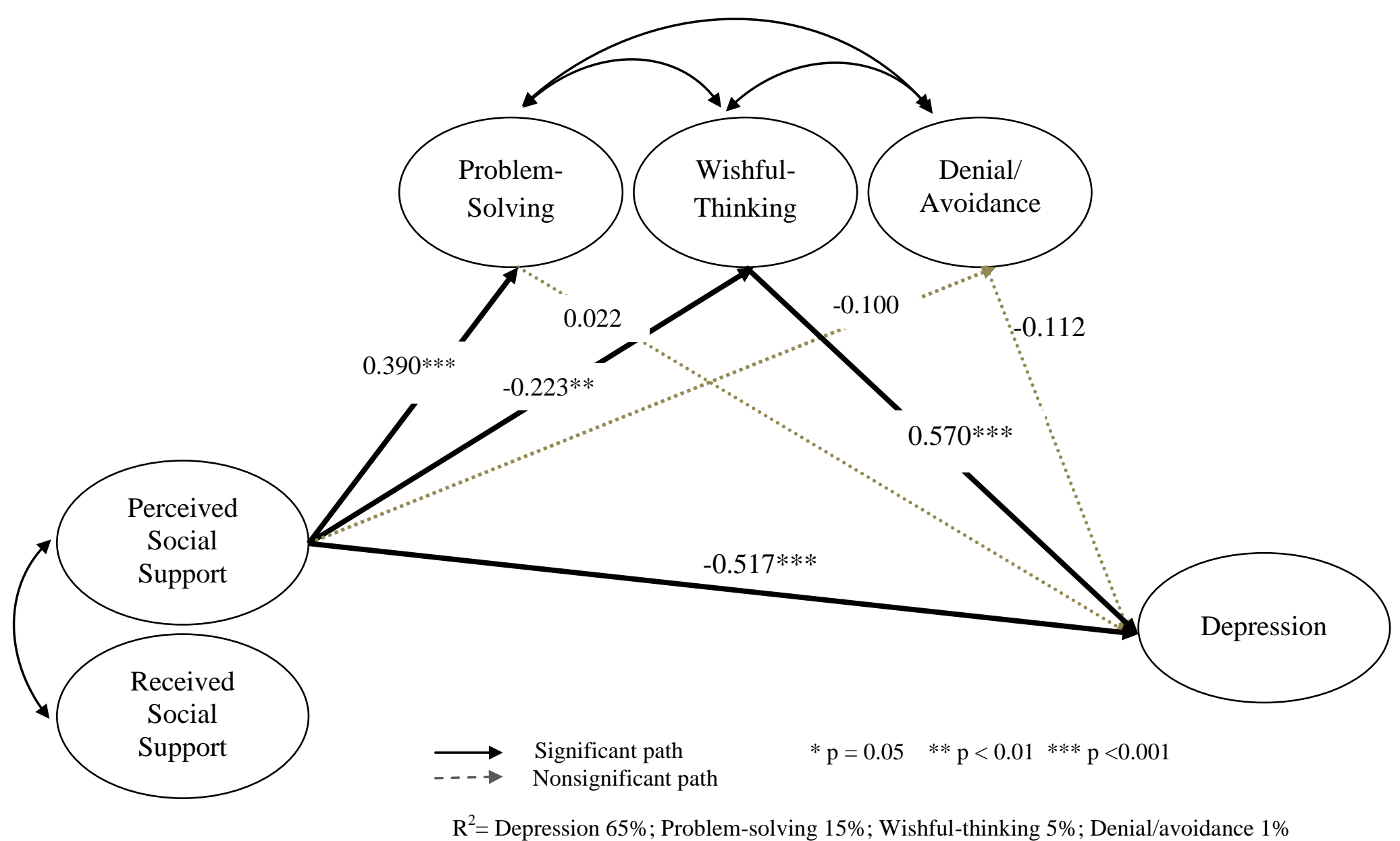


Figure 20. Model 6: The Effect of Received Social Support on Depression Mediated by Perceived Social Support

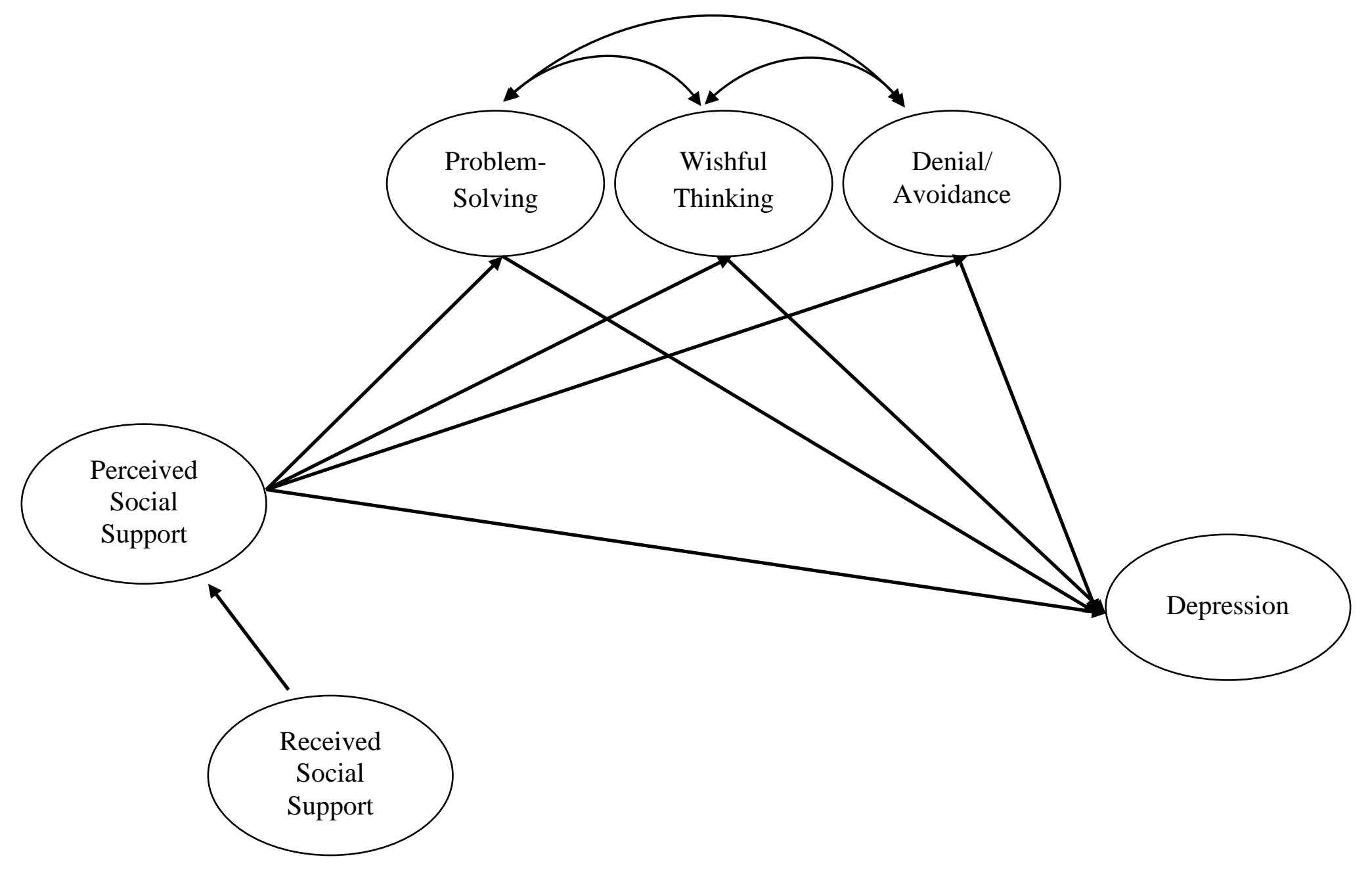


Table 22: Model 6: The Effect of Received Social Support on Depression Mediated by Perceived Social Support

\begin{tabular}{|c|c|c|c|c|c|}
\hline Path of Latent Variable & Estimate B & Estimate $\beta$ & $\begin{array}{l}\text { Standard } \\
\text { Error }\end{array}$ & P - Value & Fit Indices \\
\hline Problem-solving Coping $\rightarrow$ Depression & 0.036 & 0.022 & 0.069 & 0.750 & \multirow{8}{*}{$\begin{array}{lr}\chi 2(d f) & 1531.125(1258) \\
\text { RMSEA } & 0.028 \\
95 \% \text { CI } & 0.023-0.033 \\
\text { CFI } & 0.968\end{array}$} \\
\hline Wishful-thinking Coping $\rightarrow$ Depression & 0.691 & 0.570 & 0.079 & 0.000 & \\
\hline Denial/Avoidance Coping $\rightarrow$ Depression & -0.191 & -0.112 & 0.091 & 0.222 & \\
\hline Perceived Social Support $\rightarrow$ Depression & -0.538 & -0.517 & 0.062 & 0.000 & \\
\hline $\begin{array}{l}\text { Perceived Social Support } \rightarrow \text { Problem-solving } \\
\text { Coping }\end{array}$ & 0.245 & 0.390 & 0.065 & 0.000 & \\
\hline $\begin{array}{l}\text { Perceived Social Support } \rightarrow \text { Wishful-thinking } \\
\text { Coping }\end{array}$ & -0.191 & -0.223 & 0.067 & 0.001 & \\
\hline $\begin{array}{l}\text { Perceived Social Support } \rightarrow \text { Denial/Avoidance } \\
\text { Coping }\end{array}$ & -0.061 & -0.100 & 0.092 & 0.280 & \\
\hline $\begin{array}{l}\text { Received Social Support } \rightarrow \text { Perceived Social } \\
\text { Support }\end{array}$ & 0.768 & 0.754 & 0.046 & 0.000 & \\
\hline
\end{tabular}

$\mathrm{B}=$ unstandardized parameter estimates, $\quad \beta=$ standardized parameter estimates

$\S$ The effects of received social support on coping strategies and anxiety were constrained at 0 
depression through wishful-thinking coping strategies, and 3) received social support influences the level of depression indirectly through perceived social support (Figure 21). 
Figure 21: Significant Paths for Model 6: The Indirect Effects of Perceived Social Support on the Relationship Between Received Social Support ${ }^{\S}$ and Depression

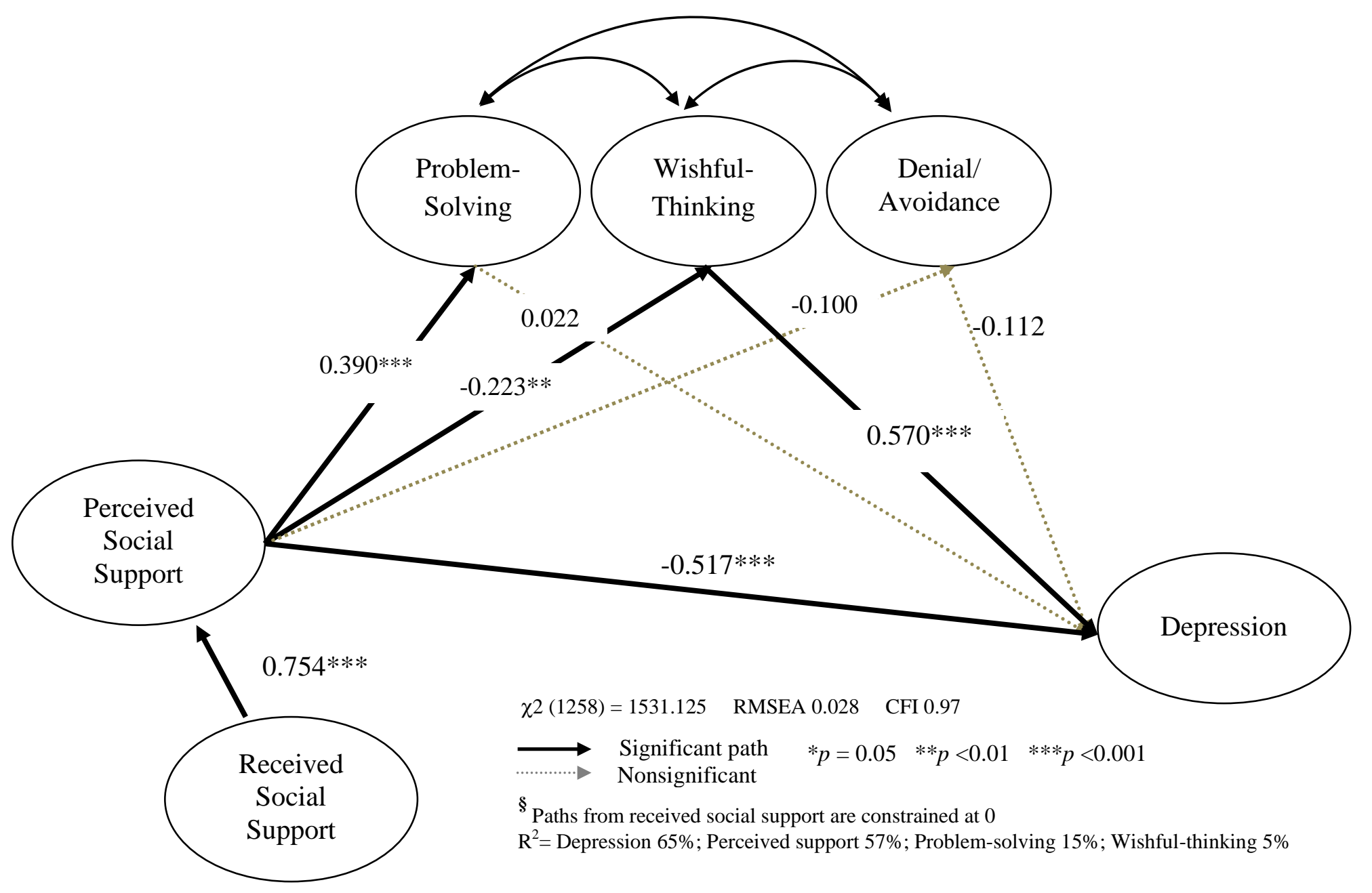




\section{Chapter 5}

\section{Discussion}

This study examined the predictors of psychological distress in a sample of adults living with congenital heart disease (CHD). The participants of this study were a relatively healthy group of individuals. More than three quarters of the sample perceived their health to be in a "good" or "very good" state. The level of their psychological distress was low, with a proportion of people reporting a moderate to severe level of anxiety and depression each, at less than $15 \%$ of the total sample. The individuals followed closely at the Adult Congenital Heart Disease (ACHD) program tend be ones with complex heart disease whereas the people with mild heart defects are not followed as closely. However, the study participants did not seem to be affected by their CHD as much as had been anticipated. The comparison of the characteristics of the participants of this study and those of other studies of individuals with CHD cannot be easily made. However, the finding that the study participants perceive themselves to be healthy and not much different from the normal population resonates with the report by Moons et al. (2006). Moons et al. described their study sample of adults with CHD as people experiencing satisfactory quality of life and who perceive their health as similar to their healthy counterpart individuals matched on demographic characteristics of age, sex, education, and occupation.

The primary purpose of this study was to ascertain the relationships between types of social support (perceived and received), types of coping strategies (problem-solving, wishful thinking, and denial/avoidance), and psychological distress of individuals with CHD. 
It was hypothesized that the social support and coping strategies of an individual influence psychological outcomes.

The key findings of this study delineate the important effects of social support on psychological distress of adults living with CHD. More specifically, a direct effect was demonstrated between perceived social support and psychological distress. Received social support also demonstrated a significant effect on psychological distress through perceived social support. That is, perceived social support mediated the relationship between received social support and psychological distress. As hypothesized, the relationship between perceived social support and psychological distress was mediated by wishful-thinking coping strategies. In contrast to our hypotheses, the relationships between types of social support and psychological distress of people with CHD were not mediated by the problem-solving or denial/avoidance coping strategies.

\section{Limitations}

Although this study contributes significantly to our knowledge regarding the psychological adaptation of individuals living with CHD, there are a number of limitations that should be noted. The cross-sectional nature of the study limits our understanding of the directionality of effects. For example, some researchers claim that psychologically distressed individuals tend to elicit more support, rather than social support offering protection from psychological distress (Coyne et al., 1981). In addition, psychologically distressed individuals may have a greater tendency to select wishful-thinking, rather than the use of wishful-thinking strategies predicting psychological distress. Despite the use of the structural equation modeling (SEM) technique which can support the plausibility of the 
proposed relationships among study variables, it is possible that a different directionality of relationships may exist. Also, the generalizability of the findings may be limited because of the convenience sample. The generalizability of the results is constrained to individuals with CHD who are members of an adult congenital heart disease (ACHD) program. Participants of this study who attended the ACHD program tended to be relatively healthy with an optimal level of functioning ranging from "fair" to "good". Thus, the findings of the study may not be generalizable to individuals who are not regularly followed by an ACHD program, or to individuals with worse functional capacity than study participants. Another limiting factor that may threaten the validity of the findings is the inadequate psychometric properties of some of the instruments used in this study, in particular, the Ways of Coping Questionnaire (WCQ). The inadequate psychometric properties of the WCQ may have precluded the detection of the true relationships among study variables. Furthermore, a lack of a separate response category for the inapplicable items of the scale may have biased the results and attenuated the strength of existing relationships. The psychometric properties of well-established instruments such as the Beck Depression Inventory could not easily be confirmed with a confirmatory factor analysis and required minor modification. The limited previous examination of psychometric properties of Berlin Social Support Scales also may have threatened the validity of the instrument. A more detailed discussion on the psychometric properties of some of the instruments is presented later. This study did not examine the potential effects of extraneous variables (e.g., age, sex, history of depression). The effects found may differ for different subgroups. Finally, this study lacked a measure for assessing the nature and appraisal (i.e., threat versus challenge) of stressors. The knowledge of the nature of the stressor is necessary for testing the buffer effect of stress and 
coping. However, the purpose of this study was the development of a predictive model of psychological distress of individuals with CHD rather than in the testing the theory of the stress of coping itself. More specifically, this study sought to ascertain the roles of social support and coping strategies in psychological adjustment. Therefore, a measure of the stressor was not included. Despite these noted limitations, the findings of this study significantly increase our understanding of the relationship between social support and coping, and their influence on psychological distress of individuals living with CHD.

\section{Discussion of the Study Findings}

In the following section, results are discussed in relation to three main areas: 1) the relationship between types of social support and psychological distress, 2) the relationship between types of social support and psychological distress mediated by types of coping strategies, and 3) the psychometric properties of the instruments used in this study.

\section{Relationship between types of social support and psychological distress.}

\section{Perceived social support.}

Cardiac patients are often faced with fear and uncertainty related to their illness and recovery, and as a result, they often experience or are at risk of developing psychological distress (Forrester et al., 1992). Evidence indicates that social support plays an integral role in influencing the psychosocial adjustment of individuals living with various chronic health challenges. The findings of this study support the role that social support plays in influencing psychological adjustment among individuals living with CHD. As hypothesized, one of the key findings of this study is the important effects of perceived social support on 
both the anxiety and depression of the study participants. Received social support also exerted an important influence but its effect was achieved through perceived social support.

Researchers have proposed three different models to illustrate the impact of social support on psychological outcomes: the direct-effect, the buffering-effect, and the indirecteffect models (Lakey \& Cohen, 2000). The direct-effect model claims that social support provides a beneficial effect on psychological outcomes regardless of whether or not stress is present. The buffering-effect model, on the other hand, asserts that social support attenuates the negative impact of stressors on psychological outcome. The indirect-effect model describes that a third variable assumes a mediating role in the relationship between social support and psychological distress. Our hypothesis that perceived social support is related to psychological outcome was supported. The finding that there is a direct relationship between social support and psychological distress is consistent with the results of previous studies involving people affected with various cardiac illnesses. For example, King, Reis, Porter, and Norsen (1993) found in their longitudinal study that emotional social support was consistently and significantly related to the emotional outcome (positive and negative moods) in 155 patients recovering from coronary artery surgery one year later. Similarly, Holahan, Moos, Holahan, and Brennan (1995) studied depressive symptoms of 325 individuals diagnosed with acute and chronic cardiac illnesses. They found that social support (confiding and understanding) had a direct relationship with depressive symptoms at oneyear follow-up. A direct effect was also observed in the study by Penninx et al. (1998) in which emotional support showed a significant relationship to depressive symptoms in older persons with chronic cardiac diseases. 
Some researchers describe social support from the perspective of a social cognition model. According to Lakey and Cohen (2000), the effect of direct social support is derived from enhanced self-esteem and self-regulation, irrespective of the presence of stress. Based on the social cognition model, perceived social support is the person's stable belief about the supportiveness of others (Lakey, Drew, Anan, Sirl, \& Butler, 2004). Perceived social support, therefore, is not based on the provision of specific helping behaviors, but rather, on summary judgments of people's behaviors including supportiveness, relationship satisfaction, providers' personality traits, and how similar providers are to the recipient of the support. Once the stable belief of perceived social support has been established, the future judgment of support is influenced by the pre-existing beliefs. Thus, the person with high perceived social support interprets the same action as more supportive, exhibits a better memory for recalling supportive behaviors, and shows a greater focus to supportive behaviors. In contrast, a person with low perceived social support interprets the same action more negatively, thereby stimulating negative thoughts about oneself, causing emotional distress (Lakey \& Cassady, 1990). Thus, it is plausible that perceived social support may function independent of the situation or the severity of stressor.

According to Cohen and Wills (1985), the buffering-effect model is more likely associated with functional measures (i.e., emotional, informational and instrumental) of support, whereas the direct-effect model is more likely associated with structural measures. The structural measures reflect the social integration (social network participation) of support which tap into more general social resources. Being embedded in social networks allows individuals to assume meaningful roles that enhance self-esteem and a sense of purpose in life. Social networks also offer a sense of physical and emotional security that serve to exert 
a direct positive effect on individuals. Although social support in the current study was assessed with the measures of perceived and received social support rather than a measure of one's social network, it may be that direct effect occurred as a result of being part of a positive social network. Barrera (1988) states that under certain circumstances, functional measures of support could be related to direct effects on mental health. Thus, individuals who are provided with a sense of self-esteem and control as a result of being embedded in a social network may be able to plan life events in order to prevent stressors in the first place. A mechanism for the direct relationship between low perceived social support and increased psychological distress is proposed by Lakey et al. (2004). They claim that people with low perceived social support tend to interpret social relationships more negatively than people with high perceived social support. Such negative interpretations, which arise from momentto-moment interpretation, are directly associated with psychological distress without influence on coping strategy.

In this study, perceived social support was inversely related to psychological distress. As stated previously, the perceived support in this study reflects primarily the function of emotional support. The perception of available support in the form of enhancing self-esteem and a sense of control may have decreased the level of anxiety and depression in this population.

\section{Received social support.}

In contrast to perceived social support, received social support did not demonstrate a direct relationship with psychological distress among individuals living with CHD. However, bivariate correlation indicated that received social support was highly correlated with perceived social support. Thus, the model was re-ran to examine the possibility that the 
effects of received social support are operating through perceived social support. In other words, perceived social support is exerting a mediating effect between received social support and psychological distress. The result of the analysis suggested that two different relationships may exist between received and perceived social support. The first suggested relationship is that received social support predicts perceived social support, which in turn, influences psychological distress. The second suggested relationship is that received and perceived social supports are highly correlated and confound one another.

The suggested relationship between received social support and psychological distress through perceived social support was an unexpected finding given the scarce research demonstrating the mediation effect of perceived support. Studies have generally reported the perceived and received social support as distinct and weakly correlated constructs wherein perceived support more consistently demonstrates a positive effect on mental health in comparison to received support (Wills \& Shinar, 2000). Typically, the benefit of perceived support is in the actual mobilization of support (received support) in times of stress. Thus, the implied direction of the relationship is perceived support predicting received support. However, the reverse direction of the relationship, meaning, received support predicting perceived support, cannot be ruled out. Although it is plausible that received social support influences psychological distress indirectly through perception of social support, a scant number of studies have actually considered this possibility (Wethington \& Kessler, 1986). Evidence for the mediation effect of perceived support between received social support and psychological distress can be found in the research focused on traumatic stress and post-traumatic stress disorder (PTSD). For example, Norris and Kaniasty (1996) studied the mediation effect of perceived social support between 
supportive behaviors (received support) and psychological distress by examining two independent groups of victims of severe natural disasters - Hurricane Hugo $(\mathrm{n}=498)$ and Hurricane Andrew ( $\mathrm{n}=404)$. They found a positive relationship between received social support and perceived social support in both samples. In the aftermath of natural disasters, individuals who received high levels of help maintained an increased level of perceived social support. The authors argued that the long term benefits of supportive behaviors on mental health are achieved indirectly through the perception that support is available if needed. A similar positive effect of received social support was reported by Wethington and Kessler (1986) who evaluated the importance of perceived social support on distress in the national survey of 1,269 adults, with the focus on married couples. The results showed that received social support from spouses operates indirectly through perceived social support to influence emotional distress. In other words, supportive behavior from spouses enhances the perception of support availability, which in turn, serves to attenuate the emotional distress of the support recipients.

As indicated previously, the findings of this study showed a significant positive relationship between received and perceived social support. This association suggests that perceptions of received and perceived social support may be influenced by different factors. Researchers suggest that unlike perceived social support, received social support is determined by a complex interplay of factors, e.g., the source, provider, type, and appropriateness of support, and its impact on the recipient (Fisher, Nadler, \& WhitcherAlagna, 1982; Lapore, Glaser, \& Roberts, 2008; Linden \& Vodermaier, 2012; Maisel \& Gable, 2009; Rook, 1984). A decreased level of received social support may arise as a result of experiencing supportive behaviors that elicit negative emotions, or those laden with 
criticism or conflicts. Merton (1968) argues that social support contains the inherent assumption of both supportive and self-threatening elements. While the supportive element conveys caring and concern for the recipient, the threatening element denotes an inferioritysuperiority relationship between the recipient and the provider, and conflicts with values of self-reliance and independence that are emphasized in western socialization (as cited in Fisher, Nadler, \& Whitcher-Alagna, 1982). Thus, when support is experienced as predominantly threatening, the reactions of the recipient are mainly negative or defensive (i.e., decreased self-concept). The recipient may interpret the support received as a reflection of personal incompetence or loss of independence. This in turn can decrease perceptions of received social support and in turn harm one's self-esteem (Fisher, Nadler, \& WhitcherAlagna, 1982) and contribute to psychological distress. Thus, it is plausible that individuals who have experienced negative support behaviors would report a decreased level of received social support.

Supportive behaviors that are plentiful but undesired likely contribute to reports of low received support and in turn, of low perceived support. For some people with chronic illnesses, received social support may be perceived as a sign of personal incompetence with managing their diseases, and influence an increased level of psychological distress. For instance, in a study involving 2810 people with various chronic illnesses (diabetes mellitus, chronic obstructive lung disease, cardiac disease, arthritis or cancer), Penninx et al. (1998) found that instrumental support was associated with depression in people with diabetes. Similarly, Helgeson (1993) reported that cardiac patients sometimes felt that their family and friends provided unsolicited support (e.g., information support) well beyond the level that was desired or needed. In a related manner, a well-intended support may fail because of an 
unhelpful action (e.g., telling the person with cancer to not worry, or be cheerful) that may only serve to undermine the person's stressor (Helgeson, 2003). The people in one's social network may try to be helpful with good intentions but provide support in an unhelpful manner either due to a lack of knowledge about the type of support desired (e.g., telling the person with complex CHD to cheer up when the person wants to express feelings of sadness), or a lack of knowledge about how to provide the needed support (e.g., avoid the person with the stressor as they do not know how to be helpful).

Finally, the "supportive" behaviors from social relationships characterized by conflict could preclude a person from interpreting a social transaction as supportive, and influence him or her to report a low level of received social support. Uchino (2004) suggests that both helpful and conflictual qualities are embedded in many close social relationships. The relationships with family and friends that provide a high level of social support can also contain elements of disputes, envy, fights, or invasion of privacy (Rook, 1984). While relationships with friends are voluntary in nature, which allow for the relationship to dissolve easily, family relationships are often based on a sense of obligation and duty and thus, cannot easily be extricated from, even when interactions are embedded with problems and conflicts (Krause, 1995). The relationship between the support provider and the support recipient can become strained and burdensome particularly if the stressor is chronic in nature and the recipient requires continual assistance in situations such as the management of chronic disease. The feelings of burden on the part of the support provider can precipitate increased feelings of resentment that the stressful event has disrupted their own lives, which in turn can result in unsupportive reactions and behaviors (Zarit, Reever, \& Bach-Peterson, 1980). Thus, people who have experienced unsupportive behaviors may not consider themselves as 
being the recipient of an increased level of received social support. Individuals who have experienced unhelpful, or negative support behaviors may be more likely to report a low, rather than high, level of received social support.

Based on the discussion above, the proposed mechanisms of social support could similarly be applied to the population affected with CHD. It is possible that perceived social support exerts a mediation effect between received social support and psychological distress. The perceived social support of the study participants could be the result of the support they have received. A number of participants with CHD in previous studies have reported the efforts of their parents to provide for them a protective and as normal an environment as possible in which to grow up. The individuals who grow up in an atmosphere of loving and supportive family members would retain an increased level of perceived support, which in turn would decrease the level of their psychological distress. In contrast, individuals who grow up in an environment characterized by conflict (Gardner, 1995) and ineffective supportive behaviors would experience a decreased perception of support availability, which in turn, would increase their psychological distress. For some individuals with CHD, being a recipient of social support may perpetuate or reinforce the "sick role" they have long assumed and may serve to undermine their own perceived capabilities. Despite good intentions, received support in such situations may be unwanted and serve to only diminish self-esteem, and decrease the perception of availability of effective social support.

Although a significant relationship was demonstrated between received social support and psychological distress through perceived social support, it is interesting to note a couple of facts. First, no direct relationship was found between received social support and psychological distress. Second, the magnitude of the correlation between received social 
support and anxiety/depression is similar in the magnitude of the correlation between perceived social support and anxiety/depression.

It is plausible that a lack of association found between received social support and psychological distress in individuals living with CHD is related to the way support was measured. Traditionally, measures of received support confound two opposing effects: the benefits when support is given and the costs experienced by the recipient when the support is received (Bolger, Zuckerman, \& Kessler, 2000). If the self-report of individuals with CHD confound the benefit and the cost of received social support, then the separate effects will not be discriminated and any relationship that may exist separately for the benefit and the cost of support will not be demonstrated. It is plausible that separate effects of the cost and benefit of support exist in this study sample. However, due to the failure of the instrument to discriminate two separate effects, any existing relationships may not have been demonstrated. Perceived social support, on the other hand, is not accompanied with a "hidden cost" and thus, the measurement does not present the problem of a confounding effect. This may be one of the reasons why we were able to discern the significant direct effect of perceived social support on psychological distress of study population in this study but not with the received social support.

Another unexpected finding was related to the similar magnitude of correlations between each of received and perceived social support and anxiety/depression. In addition, contrary to other study findings that indicate perceived social support is moderately correlated with received social support, the results of this study showed that two variables are highly correlated. Although the analysis suggests that perceived social support may mediate the relationship between received social support and anxiety/depression, other explanations 
may also account for the relationship. The alternative explanation for this finding may be that perceived and received social support are confounded with one another. For individuals living with $\mathrm{CHD}$, the distinction between perceived and received social support may not be as clearly distinguishable as it is for individuals coping with new diagnoses or with escalating chronic conditions. In particular, people with CHD who have grown up constantly receiving various types of social support (i.e., physical assistance, frequent medical visits, emotional protection, etc.) may not be able to differentiate the assistance they receive from perceptions that the assistance is helpful. In such situations, it would be difficult to ascertain their separate effects on the outcomes of anxiety/depression. Because perceived and received social support were confounded, further studies are needed to differentiate the separate effect of the constructs.

\section{Relationship between types of social support and psychological distress mediated by different coping strategies.}

\section{Perceived social support and coping strategies.}

Perceived social support was inversely related to the coping strategies of wishfulthinking. That is, individuals with a greater level of perceived social support tend to engage in lesser amount of wishful-thinking coping strategies. Researchers claim that social support encourages increased use of adaptive coping behaviors (e.g., "Talked to someone who could do something concrete about the problem") and discourages the use of maladaptive coping behaviors (e.g., wished that the situation would go away or somehow be over with) (Thoits, 1995). Subsequently, a decreased use of wishful-thinking coping strategies was associated with decreased levels of both anxiety and depression; alternatively, a greater tendency to 
engage in wishful-thinking coping strategies was related to increased levels of anxiety and depression. A limited number of studies have examined the relationship between perceived social support and the use of wishful-thinking coping strategies. The existing evidence indicates that perceived social support has a positive association with adaptive coping strategies (i.e., problem-focused coping). Researchers claim that the perception of support availability encourages a person to pursue and engage in practical resolution of their problems rather than avoiding the stressor (Wethington \& Kessler, 1986). In situations of decreased perceived support, a person may experience a reduced perceived ability to handle the stressor that may cause him or her to engage in wishful-thinking coping strategies in an attempt avoid feeling psychological distress. Indeed, the substantiation for this conjecture is supported in a study of Appalachian adolescents. Markstrom, Marshall and Tryon (2000) studied 113 Appalachian high school students and found that perceived family social support was inversely related to the use of wishful-thinking coping strategies. Thus, as the perception of social support decreased, students tended to engage in more of wishfulthinking coping strategies. Green and Pomeroy (2007) examined the effect of social support in victims of violent and nonviolent crime and found that perceived social support was negatively related to avoidance-oriented coping and positively related to problem-focused coping strategies. Given the general agreement in the literature that both avoidance and wishful-thinking coping strategies are maladaptive and prevent a person from resolving problems, it is reasonable to assume that people with decreased perceived support would also likely use wishful-thinking coping strategies. 


\section{Coping strategies and psychological distress.}

Vollman, LaMontagne and Hepworth (2007) studied 75 adults living with heart failure and found that individuals who used more escape types of coping (e.g., wishful thinking) experienced more depressive symptoms, whereas individuals who used more problem-solving coping strategies experienced fewer depressive symptoms. The negative effect of the wishful thinking coping strategies on psychological outcomes has been consistently demonstrated across populations with different chronic illnesses. For example, Felton and Revenson (1984) conducted a longitudinal study to examine the coping strategies of adults ( $\mathrm{N}=151)$ affected with four different types of chronic illnesses: hypertension, diabetes, rheumatoid arthritis, and cancer. They found that the coping strategies of wishfulfilling fantasy were significantly associated with negative affect (e.g., feelings of uselessness). In contrast, the coping strategies of problem-solving (e.g., information seeking) was related to positive affect. Similar findings were reported for people with multiple sclerosis (McCabe, McKern, \& McDonald, 2004) and breast cancer (Stanton \& Snider, 1993).

The coping strategies of wishful thinking could be thought of as a type of experiential avoidance, which reflects an individual's opposition to experience undesired thoughts, emotions, or somatic sensations, and his or her responsive attempts to alter, avoid, or escape from those experiences (Plumb, Orsillo and Luterek, 2004). Wishful thinking is a tactic used to divert the focus away from the realities of the circumstance, to ruminate about what might have been and to satisfy a self-indulging desire for better times when there seems to be no escape from the stressor. Wishful rumination reinforces feelings of unhappiness and the difficulty of accepting a stressful situation. It also erodes positive psychological outcomes (Felton \& Revenson, 1984). Coyne, Aldwin and Lazarus (1981) claim that 
individuals engaged in the coping strategies of wishful thinking dwell on their perceived inability to become stronger or change the way they feel. Furthermore, by rehashing their problems over and over again, individuals punctuate the negative features of their situations that subsequently serve to reduce the decisiveness and effectiveness with which they cope with the stressor.

The findings of this study are congruent with reports focused on the experiences of people who have grown up with CHD. Persons with CHD have expressed a sense of despair from their inability to keep up with their peers during their participation in sports, which in turn, may have caused them to feel inferior and impede their ability to achieve positive emotional outcomes (Claessens et al., 2005). For these distressed individuals who perceived themselves as being on an unequal footing with their peers, the choice of coping strategy may have been to fantasize of a better place where the inequality in physical capacity did not exist.

The lack of association found between the remaining coping strategies of denial/avoidance and psychological distress is puzzling especially given the existing evidence of a positive relationship between denial and depression among cardiac patients (Dew et al., 1996; Doering et al., 2004). Some researchers have suggested that in certain circumstances, denial/avoidance may serve as adaptive coping strategies (Levine et al., 1987). In the initial phase of a stressor, if a person is too emotionally overwhelmed to effectively deal with the problem, then the denial/avoidance tactic may provide a temporary refuge in which the person could assuage the emotional distress and obtain the necessary respite to engage in adaptive coping (Benotsch et al., 2000). But this strategy is effective only for a short-term (Suls \& Fletcher, 1985). Therefore, it is plausible that in situations involving 
adults with CHD, the lack of an observed relationship between psychological distress and denial/avoidance may reflect that the chronic nature of the stressor for which an escape type of coping strategies is no longer effective.

Although the bivariate correlation between received social support and the use of problem-solving coping strategies was moderate $(r=0.31)$, the relationship was not significant in the SEM. The bivariate result is consistent with findings from previous studies. For example, Dunkel-Schetter, Folkman, \& Lazarus (1987) reported that received informational, instrumental, and emotional supports were positively associated with problem-focused coping, while a decreased level of informational support was associated with the use of emotion-focused coping. Lazarus and Folkman (1984) conceptualized social support as a resource an individual draws on in order to cope with a stressor. They argue that social support is an antecedent variable that influences coping. Support in the form of advice and encouragement from a confidant may increase the probability that a person will engage in positive coping strategies such as information seeking, or active problem-solving (Valentiner, Holahan, \& Moos, 1994). Received social support did not demonstrate any significant association with other coping strategies, e.g., wishful thinking and denial/avoidance. In contrast to the relationship between positive social support and coping strategies, the mechanism for the relationship between decreased or negative social support (i.e., criticism, conflict) and coping strategies has not been studied extensively. Nonetheless, decreased levels of social support or possibly, the negative nature of social support is thought to encourage maladaptive coping strategies such as wishful thinking or cognitive avoidance (Manne, Pape, Taylor, \& Dougherty, 1999), perhaps because of an individual's intense desire to "escape" from the unpleasant reality of a stressor. 
Notwithstanding the bivariate correlation between received social support and problem-solving coping strategy, given the lack of relationships between received social support and coping strategies in the SEM, it is conceivable that the use of maladaptive coping strategies such as wishful thinking or avoidance/denial measured in individuals with CHD may operate independently from types of social support. That is, factors other than received social support may exert greater influence on an individuals' choice of coping. DeLongis and Holtzman (2005) claim that three contextual factors influence one's probability of engaging in certain types of coping strategies: 1) the nature of stressful event, 2) the social context in which coping occurs (e.g., marital satisfaction, and 3) the personality of the person (e.g., neuroticism). For example, in relation to the nature of a stressful event, the choice of coping strategies may change depending on the duration of that event. During the acute phase, a person may use denial to deal with an overwhelming sense of distress, however after a period of adjustment, that same person may go on to choose a problemfocused strategy (e.g., come up with possible solutions) to deal with the stressor. Clearly, the choice of coping strategies is influenced by multiple factors that may change according to the context of the stressor. In order to better understand the coping behavior of individuals living with CHD, other influencing factors including those outlined by DeLongis and Holtzman should be studied in future research.

\section{Validity of instruments.}

One of the major challenges associated with this study was dealing with the unsatisfactory psychometric properties of the instruments, in particular, the Ways of Coping Questionnaire (WCQ). In fact, most of the current, available coping instruments exhibit a 
variety of psychometric inadequacies (Parker \& Endler, 1992). Coping researchers face at least two major problems related to coping instruments. First, a plethora of coping measures assess different constructs, which challenges our ability to make generalizations across studies and populations. Second, coping scales have problematic psychometric properties preventing researchers from obtaining valid and generalizable information about coping behavior (Parker \& Endler, 1992). Researchers have proposed that psychometric problems with coping instruments pertain primarily to three areas: 1) stability, 2) dimensionality, and 3) inapplicable items. Of these three areas, the factors applicable to this study were the dimensionality and the inapplicable items. Stability represents "pattern similarity of interindividual differences" at several different points in time (Schwarzer \& Schwarzer, 1996). The emphasis of empirical studies is on ascertaining individual differences. When coping is assessed with standardized instrument, the inherent assumption is that people can be characterized by their favoured way of coping with the stressor, and that they use similar coping strategies over time. Such an assumption reflects a dispositional approach. However, the dispositional approach used for assessing inter-individual differences is inconsistent with the situational (contextual) coping approach, which focuses on intra-individual differences, implied in the stress and coping theory. Lazarus and Folkman (1984) argue that people choose coping strategies based on their own resources and the demands of a situation (Sorlie \& Sexton, 2001). Thus, according to the situational approach, coping is a dynamic process that changes according to the circumstances of the stressor. The major problem with the situational approach is the stability of its measures (Parker, Endler, \& Bagby, 1993; Scherer, Wiebe, Luther, \& Adams, 1988). When the instrument is unstable, factor stability cannot be established and the ways of coping concept have very little meaning. Establishment of factor 
stability requires a search for similar people/situation contexts as well as differences among dissimilar contexts (Scherer et al., 1988). Evidence indicates that people may alternate between different types of coping strategies depending on the duration and the trajectory of the stressor (Linden \& Vodermaker, 2012). In order to accurately capture the dynamic process of coping that is consistent with the contextual coping approach, the data collection method of a daily assessment of coping has been proposed (DeLongis \& Holtzman, 2005; Manne, Pape, Taylor, \& Dougherty, 1999). DeLongis and Holtzman claim that the effects of using a particular coping strategy or form of social support may be short lived and dissipate within days. Using the "daily process methodology" (day-to-day monitoring) enables researchers to capture rapidly fluctuating coping processes. For example, a person may use denial/avoidance coping to deal with a stressor at the onset of a severe, uncontrollable problem (i.e., severe burn), and then change to more problem-solving coping strategies (i.e., active physical therapy) at the later recovery stage.

The failure to detect the significant mediating effect of the remaining coping strategies (problem-solving and denial/avoidance) on the relationship between social support and psychological distress in the current study of individuals with CHD may have resulted from the failure to capture the dynamic interactive processes among these variables that unfold in a temporal sequence.

The second problem of psychometric properties pertains to dimensionality. Dimensionality refers to categorizing a set of coping strategies based on their purpose, meaning, and functional objectives (Schwarzer \& Schwarzer, 1996). Empirically, the dimensions of the measure can be established with a factor analysis. However, researchers using the WCQ have frequently reported that the factor solutions differ from sample to 
sample and from stressor to stressor. Researchers have extracted a varied number of factors ranging from two to eight that have rarely been replicated. They have also noted subscales with similar names that often included different sets of items. The wide variability of factor structure has led some researchers to recommend that each researcher using the WCQ should perform factor analyses on their own data and then use the results to ascertain the subscale structure for the coping items (Parker, Endler, \& Bagby, 1993). Indeed, this is exactly what was required in current study. Following the numerous unsuccessful attempts at confirmatory factor analyses of the WCQ based on previously identified factor structures, the decision was made to conduct an exploratory factor analysis to determine the latent factor structure of the scale.

The third problem with the WCQ instrument pertains to the inapplicable items in the instrument. The problem of replicating factor structure may be attributed to items that are not applicable for certain individuals in specific situations. For example, the item "I jogged or exercised" would not be one of the possible coping strategies for an individual who became paralyzed as a result of a motor vehicle accident. Waller (1989) claims that in the presence of 5\% inapplicable items, the bivariate correlation consisting of one inapplicable item will reduce the $\mathrm{R}^{2}$ by $14 \%$. In other words, the presence of a small to moderate percentage of inapplicable items could seriously obfuscate analyses. Richaud de Minzi and Sacchi (2001) indicate that the failure to select an item due to one's disinclination to use the strategy is different from the failure to select the same item because of its inapplicability to the stressor. Thus, the failure to discriminate between the two situations could result in a distorted or invalid interpretation of the ways in which individuals actually cope with the stressor. The WCQ is especially vulnerable to the critical issue of inapplicable items (Parker, 
Endler, \& Bagby, 1993). One way to rectify this issue is to provide a separate category for the inapplicable response (Waller, 1989). Unfortunately, the current study did not provide a separate category for inapplicable items and this is one of its limitations.

Based on the discussion above, it is plausible that the failure to detect significant mediating effects of both problem-solving and denial/avoidance coping strategies on the association between social support and psychological distress may be related to the inadequate psychometric properties of WCQ.

The psychometric issues were also present for other instruments used in this study: the Beck Anxiety Inventory (BAI), the Beck Depression Inventory (BDI-II) and the Berlin Social Support Scales (BSSS). For example, the factor structure of the BAI has been examined by different studies that resulted in a varied number of factors ranging from two to five. Using the data from current study participants with CHD, various factor structures described in the literature were tested. Only the one model described in the literature showed a marginally good fit with the data. The second-order model of four-factor structure (neurophysiological, subjective, panic, and autonomic) proposed by Osman et al. (1997) was the most promising. Since the focus of this study was to capture the subjective dimension of anxiety, the subjective subscale was the only measure included in this analysis. Thus, it is possible that the remaining dimension of anxiety excluded from the study may not have been captured. A similar challenge of the psychometric properties was observed with the BDI-II. Of the various numbers of factors tested with the current ACHD data, only a two-factor model proposed by Whisman, Perez, \& Ramel (2000) showed a good fit. Since the purpose of the study was to capture the cognitive-affective aspect free of physical symptoms, only the cognitive subscale was included. Finally, although the BSSS is a measure that is brief and 
able to assess multifaceted dimensions of social support, it is a relatively new scale that lacks extensive examination of factor structures. The factor analysis of the perceived social support subscale demonstrated a poor fit with the data when tested for unidimensionality (i.e., perceived social support). One of the advantages of the BSSS is that the subscale of perceived support can be further categorized into emotional and instrumental support based on the function of support. When factor structures were tested for two latent factors (emotional and instrumental), the result showed a good fit with the data. However, a failure to demonstrate a good fit for unidimensionality of the measure may have prevented the capture of instrumental aspect of perceived social support.

In summary, the various psychometric issues associated with the instruments, including ones that have been extensively used in the literature, are a potential challenge to the validity of this study. The ability to examine the psychometric properties of instruments and estimate relationships among constructs accounting for the random errors of the measures is a great advantage offered by the sophisticated analytical techniques (e.g., SEM) available today. The development of increasingly advanced analytic procedures illuminates the rigorous and complex endeavours necessary for developing a sound instrument that can measure the construct being researched. The findings of this study illustrate the need for careful examination of psychometric properties and the need for a replication of factor structures to firmly establish the validity of measures used in future studies.

\section{Implications}

The findings of this study extend our understanding of how types of social support, coping strategies and psychological distress relate to each other in individuals living with 
CHD. Results of this study present several implication for nurses caring for this unique group of individuals faced with chronic health challenges. Perceived social support was observed to exert a significant impact on anxiety and depression in this group. Thus, the assessment of social support should continue to be an integral part of nursing care. In particular, since the perceived support pertaining to the function of emotional support was highlighted in the results of this study, the assessment should focus on the emotional aspect of support. Evidence suggests that by virtue of their relationships to the recipient, life experiences and their own resources, different members of a person with CHD's network may be equipped to provide different types of support. It follows then, that the assessment and identification of people perceived by individuals with CHD to provide emotional support is imperative. The finding of this related to received social support suggests that one's prior experience with supportive behavior from others shapes one's future perception of support availability. Given the results of previous studies identifying the negative effect of supportive behaviors characterized by criticism or conflictual relationships, nursing care should focus on assessing the quality of social relationships the person maintains with members of his or her social network. Findings of social support imply that nursing intervention could be approached from two different angles with concentrated efforts targeting the received and perceived social supports separately. The intervention that addresses the specific areas of concern would have a greater chance for the optimal outcome. Cohen, Underwood, and Gottlieb (2000) emphasize that support is a reflection of a personal relationship that is characterized by "sense of attachment, intimacy, mutuality, and solidarity". They further claim that the meaning of supportive behavior of others is derived from social relationships. For people with CHD, the benefit would be more pronounced if 
the shaping of one's perception of support starts early in life, i.e., childhood. The relationships characterized by support and security would positively shape a person with CHD's perception of the supportiveness of others and help interpret social interaction in a more favorable light thereby decreasing their emotional distress.

In terms of received support, evidence suggests that the manner of support provision that creates the least amount of negative influence is invisible support. That is, when the recipient is unaware that the support is given, then the degree of negative impact appears to be less. Thus, the provider could be encouraged and taught to deliver the support in an inconspicuous manner. In addition, as the effect of negative social interaction has just as much impact, if not more, on psychological distress, people with CHD should be encouraged to either extricate themselves from relationships with conflict or to avoid the source of harmful negative relationship and to develop new relationships. The new relationships should be characterized by frequent and sufficient contacts that foster the development of intimacy and mutual sharing.

The relationship between perceived social support and psychological distress was mediated by the coping strategies of wishful-thinking. This implies that individuals with a decreased level of perceived social support tend to favor the use of wishful-thinking coping strategies and are at a higher risk for experiencing psychological distress. For example, evidence suggests that problem-focused coping is more effective for stressors that are deemed controllable, whereas emotion-focused coping is more beneficial for stressors that are deemed uncontrollable (e.g., terminal cancer). Thus, the person could be encouraged to carefully examine and appraise the stressor appropriately, and to choose either problem- or emotion-focused coping strategies that can effectively address the stressor. Further, people 
with low perceived support should be discouraged from using wishful-thinking coping strategies and instead, incited to use more stressor appropriate adaptive coping strategies (i.e., problem-focused coping).

In terms of directions for future research, one of the major concerns of coping research is the lack of a measure that can comprehensively reflect the concepts delineated in the stress and coping theory. Although the coping theory by Lazarus has served to greatly increase our understanding of the dynamic nature of coping processes, the corresponding coping measures fall short in their ability to capture the dimensions of coping, primarily due to its unstable factor structures. Researchers claim that coping scales were developed by empirical consideration, rather than being guided by theory, which resulted in a plethora of coping measures with multiple unstable factor structures (Alwin \& Revenson, 1987). Consequently, little consensus exists for the conceptualization and measurement of coping strategies. The multiple proposed factor structures for the same measure raise questions about the reliability and validity of coping instruments and threaten the accuracy of study findings.

The results of this study challenge our thinking about nursing research in the area of coping. Although the outcome measure of this study was emotional distress, perhaps some of the coping strategies are associated with the positive psychological outcomes. For example, as discussed previously, evidence suggests that problem-focused coping strategies are related to positive psychological outcomes. Had this study measured positive emotional outcomes, a significant relationship may have been demonstrated. It is also possible that some of the coping strategies that people with CHD use are not part of the items of the instrument. For instance, the coping strategy of comparing one's situation in a more 
favorable light than other individuals facing the similar stressor is not one of the items included in the scale. Additional studies are needed to further examine and identify the exact factors and mechanisms that influence psychological outcomes in this population.

On a conceptual level, findings of this study encourage nursing researchers to explore beyond the constructs of coping. It is plausible that coping strategies may not be the most important predictor of psychological distress in individuals living with CHD. Researchers have described different variables that can influence psychological outcomes such as perceived control (Moser \& Dracup, 1995), self-efficacy (Penninx et al., 1998; Shnek, Irvine, Stewart, \& Abbey, 2001; Tsay \& Chao, 2002) and coping efficacy (Aldwin \& Revenson, 1987). It may be worthwhile to explore variables other than the coping strategies to discern their influence on psychological distress. In terms of social support, future research could examine the relationship between different functions of social support (i.e., emotional, instrumental, and information) and psychological distress of adults living with CHD. It is possible that different functions of social support may relate differently to psychological distress. Each of social function may relate differently to different types of coping strategy, which in turn, exert influence on psychological distress.

The scope of this study did not allow us to specifically identify those individuals experiencing a high level of psychological distress who are most in need of an intervention. Prior to developing interventions for this group of highly distressed individuals, the crucial step would be to clearly understand the nature of their distress and its influencing factors. Qualitative methods could be used to engage in in-depth exploration of issues facing the distressed subgroup and could provide an important milestone that would help us to learn how best to help this highly distressed group of adults. 
A next important step is to replicate this study using different measures of coping. For instance, the development of an instrument specific for the CHD population may be useful to increase our understanding of how this group of people deal with stressors related to their chronic illness. Research that uses the data collection method of day-to-day monitoring of coping strategies may be a good way to capture the coping behaviors that unfold in temporal sequences across different situations. The data would serve to help design interventions that are specific in dealing with stressors experienced by individuals living with CHD.

\section{Conclusion}

The research on the psychological adjustment of individuals living with CHD is still at the rudimentary stage of knowledge development. However, this study illuminates our understanding about the effect of social support and coping strategies as predictors of psychological distress. Despite numerous theories on how social support operates, we are still not clear about its exact intervening mechanism in the context of CHD. Similarly, the process of coping with stress is far more complex and intricate than some may have envisioned. One of the major research challenges in the field of stress and coping is in the development of a stable measure that has the ability to detect true relationships among study variables. Further studies need to be conducted to refine and validate existing coping measures to establish sound psychometric properties. Furthermore, studies that explore the relationship between different functions of social support (i.e., emotional, instrumental, and informational) and different coping strategies would be beneficial to clarify the underlying 
mechanisms, which in turn would allow us to better target interventions that promote the psychological adjustment of individuals living with CHD. 


\section{References}

Abbey, A., Abramis, D. J., \& Caplan, R. D. (1985). Effects of different sources of social support and social conflict on emotional well-being. Basic \& Applied Social Psychology, 6(2), 111-129.

Aben, I., Verhey, F., Lousberg, R., Lodder, J., \& Honig, A. (2002). Validity of the beck depression inventory, hospital anxiety and depression scale, SCL-90, and Hamilton depression rating scale as screening instruments for depression in stroke patients. Psychosomatics, 43(5), 386-393.

Abramson, L. Y., Alloy, L. B., Haeffel, G. J., MacCoon, D. G., \& Gibb, B. E. (2002). Cognitive vulnerability-stress models of depression in a self-regulatory, psychological context. In I. H. Gotlib, \& C. L. Hammen (Eds.), Handbook of depression. New York: Guilford.

Adsett, C. A., \& Bruhn, J. G. (1968). Short-term group psychotherapy for post-myocardial infarction patients and their wives. The Canadian Medical Association Journal, 99, $577-$ 584.

Aldwin, C. M., \& Revenson, T. A. (1987). Does coping help? A reexamination of the relation between coping and mental health. Journal of Personality and Social Psychology, 53(2), 337-348.

Arnau, R. C., Meagher, M. W., Norris, M. P., \& Bramson, R. (2001). Psychometric evaluation of the beck depression inventory-II with primary care medical patients. Health Psychology, 20(2), 112-119.

Atkinson, M., \& Violato, C. (1993). A factor analysis of the ways of coping questionnaire based on data from saddening experiences. Psychological Reports, 72(3), 1159-1164.

Auerbach, S. M. (1989). Stress management and coping research in the health care setting: An overview and methodological commentary. Journal of Consulting and Clinical Psychology, 57(3), 388-395.

Bandura, A. (1982). Self-efficacy mechanism in human agency. American Psychologist, 37(2), 122-147. 
Barnett, R. C. (1993). Mulitple roles, gender, and psychological distress. In L. Goldberger, \& S. Breznitz (Eds.), Handbook of stress: Theoretical and clinical aspects. (2nd ed., pp. 427-445). New York: Free Press.

Barrera, M. (1988). Models of social support and life stress: Beyond the buffering hypothesis. In L. Cohen (Ed.), Life events and psychological functioning: Theoretical and methodological issues. (pp. 211-236). London: Sage.

Beck, A. T., Epstein, N., Brown, G., \& Steer, R. A. (1988). An inventory for measuring clinical anxiety: Psychometric properties. Journal of Consulting and Clinical Psychology, 56(6), 893-897.

Beck, A. T., Steer, R. A., Ball, R., \& Ranieri, W. F. (1996). Comparison of beck depression inventories - 1A and -II in psychiatric outpatients. Journal of Personality Assessment, 67(3), 588-597.

Beck, A. T., Steer, R. A., \& Brown, G. K. (1996). Manual for beck depression inventory-II. San Antonio, TX.: Psychological Corporation.

Beck, A. T., Ward, C. H., Mendelson, M., Mock, J., \& Erbaugh, J. (1961). An inventory for measuring depression. Archives of General Psychiatry, 4, 561-571.

Beck, C. T., \& Gable, R. K. (2001). Comparative analysis of the performance of the postpartum depression screening scale with two other depression instruments. Nursing Research, 50(4), 242-250.

Beck, A. T., Epstein, N., Brown, G., \& Steer, R. A. (1988). An inventory for measuring clinical anxiety: Psychometric properties. Journal of Consulting and Clinical Psychology, 56(6), 893-897.

Beck, A. T., Steer, R. A., \& Carbin, M. G. (1988). Psychometric properties of the beck depression inventory: Twenty-five years of evaluation. Clinical Psychology Review, $8(1), 77-100$.

Benotsch, E. G., Brailey, K., Vasterling, J. J., Uddo, M., Constans, J. I., \& Sutker, P. B. (2000). War zone stress, personal and environmental resources, and PTSD symptoms in gulf war veterans: A longitudinal perspective. Journal of Abnormal Psychology, 109(2), 205-213. 
Bentler, P. M., \& Chou, C. (1987). Practical issues in structural modeling. Sociological Methods and Research, 16(1), 78-117.

Billings, A. G., \& Moos, R. H. (1981). The role of coping responses and social resources in attenuating the stress of life events. Journal of Behavioral Medicine, 4(2), 157-189.

Blatt, S. J. (2004). Two types of depression. In S. J. Blatt (Ed.), Experiences of depression: Theoretical, clinical and research perspectives (pp. 15-52). Washington, DC: American Psychological Association.

Blatt, S. J., D’Afflitti, J. P., \& Quinlan, D. M. (1976). Experiences of depression in normal young adults. Journal of Abnormal Psychology, 85, 107-157.

Blumenthal, J. A. (1982). Assessment of patients with coronary heart disease. In F. J. Keefe, \& J. A. Blumenthal (Eds.), Assessment strategies in behavioral medicine (pp. 37-97). New York: Grune \& Stratton.

Blumenthal, J. A., Lett, H. S., Babyak, M. A., White, W., Smith, P. K., Mark, D. B., . . Newman, M. F. (2003). Depression as a risk factor for mortality after coronary artery bypass surgery. Lancet, 362(9384), 604-609.

Bjornstad, P. G., Spurkland, I., \& Lindberg, H. L. (1995). The impact of severe congenital heart disease on physical and psychosocial functioning in adolescents. Cardiology in the Young, 5(1), 55-62.

Boehmer, S., Luszczynska, A., \& Schwarzer, R. (2007). Coping and quality of life after tumor surgery: Personal and social resources promote different domains of quality of life. Anxiety, Stress, and Coping, 20, 61-75.

Bolger, N., Zuckerman, A., \& Kessler, R. C. (2000). Invisible support and adjustment to stress. Journal of Personality and Social Psychology, 79(6), 953-961.

Borden, J. W., Peterson, D. R., \& Jackson, E. A. (1991). The beck anxiety inventory in nonclinical samples: Initial psychometric properties. Journal of Psychopathology and Behavioral Assessment, 13, 345-356.

Bosley, C. M., Fosbury, J. A., \& Cochrane, G. M. (1995). The psychological factors associated with poor compliance with treatment in asthma. European Respiratory Journal, 8, 899-904. 
Botelho, R. J., \& Dudrak, R. (1992). Home assessment of adherence to long-term medication in the elderly. Journal of Family Practice, 35, 61-65.

Bouchard, G., Sabourin, S., Lussier, Y., Wright, J., \& Richer, C. (1997). Testing the theoretical models underlying the ways of coping questionnaire with couples. Journal of Marriage and Family, 59(2), 409-418.

Brandhagen, D. J., Feldt, R. H., \& Williams, D. E. (1991). Long-term psychologic implications of congenital heart disease: a 25-year follow-up. Mayo Clinic Proceedings, 66(5), 474-479.

Broadhead, W. E., Kaplan, B. H., James, S. A., Wagner, E. H., Schoenback, V. J., Grimson, R., Heyden, S. T., \& Gehlback, S. H. (1983). The epidemiologic evidence for a relationship between social support and health. American Journal of Epidemiology, 117, 521-537.

Bromberg, J. I., Beasley, P. J., D'Angelo, E. J., Landzberg, M., \& DeMaso, D. R. (2003). Depression and anxiety in adults with congenital heart disease: A pilot study. Heart \& Lung, 32(2), 105-110.

Brown, B. W., Andrews, B., Harris, T., Adler, Z., \& Bridge, L. (1986). Social support, selfesteem, and depression. Psychological Medicine, 16, 813-831.

Brown, T. A. (2006). Confirmatory factor analysis for applied research. New York: Guildford Press.

Browne, M. W., \& Cudeck, R. (1993). Alternative ways of assessing model fit. In K. A. Bollen, \& J. S. Long (Eds.), Testing structural equation models. (pp. 136-162). Newbury Park, CA: Sage.

Brummet, B. H., Barefoot, J. C., \& Siegler, I. C. (2001). Characteristics of socially isolated patients with coronary artery disease who are at elevated risk for mortality. Psychosomatic Medicine, 63, 267-272.

Burker, E. J., Evon, D. M., Losielle, M. M., Finkel, J. B., \& Mill, M. R. (2005). Coping predicts depression and disability in heart transplant candidates. Journal of Psychosomatic Research, 59, 215-222. 
Burker, E. J., Evon, D. M., Sedway, J. A., \& Egan, T. (2004). Religious coping, psychological distress and disability among patients with end-stage pulmonary disease. Journal of Clinical Psychology in Medical Setting, 11, 178-193.

Bussing, A., \& Fisher, J. (2009). Interpretation of illness in cancer survivor is associated with health-related variables and adaptive coping styles. BMC Women's Health, 9(2), doi:10.1186/1472-6874-9-2.

Byrne, B. M. (1998). Structural equation modeling with LISREL, PRELIS, and SIMPLIS: Basic concepts, applications, and programming. Mahwah, NJ: Lawrence Erlbaum Associates.

Byrne, B. M. (2012). Structural equation modeling with Mplus: Basic concepts, applications, and programming. New York: Routledge.

Byrne, M. B. (2001). Structural equation modeling with AMOS. Mahwah, NJ: Lawrence Erlbaum Associates, Inc.

Camey, R. M., Freedland, K. F., Fisen, S. E., Rich, M. W., \& Jaffe, A. S. (1995). Depression is associated with poor adherence to medical treatment regimen in elderly cardiac patient. Health Psychology, 14, 88-90.

Carver, C. S., Scheier, M. F., \& Weintraub, J. K. (1989). Assessing coping strategies: A theoretically based approach. Journal of Personality and Social Psychology, 56(2), $267-$ 283.

Case, R. B., Moss, A. L., Case, N., McDermitt, M., \& Aberly, S. (1992). Living alone after myocardial infarction: Impact on prognosis. JAMA, 267, 515-519.

Cetta, R., Graham, L. C., Lichtenberg, R. C., \& Warnes, C. A. (1999). Piercing and tattooing in patients with congenital heart disease: Patient and physician perspectives. Journal of Adolescent Health, 24(3), 160-162.

Chan, D. W. (1994). The Chinese ways of coping questionnaire: Assessing coping in secondary school teachers and students in Hong Kong. Psychological Assessment, 6(2), 108-116.

Chaudron, L. H., Szilagyi, P. G., Tang, W., Anson, E., Talbot, N. L., Wadkins, H. I. M., . . . Wisner, K. L. (2010). Accuracy of depression screening tools for identifying postpartum depression among urban mothers. Pediatrics, 125(3), e609-e617. 
Child, J. (2001). Care of the adult with congenital heart disease. Presented at the 32nd Bethesda Conference, Bethesda, Maryland October 2-3, 2000.

Claessens, P., Moons, P., de Casterle, B. D., Cannaerts, N., Budts, W., \& Gewillig, M. (2005). What does it mean to live with a congenital heart disease? A qualitative study on the lived experiences of adult patients. European Journal of Cardiovascular Nursing, 4, 3-10.

Cohen, M., Mansoor, D., Langut, H., \& Lorber, A. (2007). Quality of life, depressed mood, and self-esteem in adolescents with heart disease. Psychosomatic Medicine, 69, 313-318.

Cohen, S. (1988). Psychosocial models of the role of social support in the etiology of physical disease. Health Psychology, 7(3), 269-297.

Cohen, S., Mermelstein, R. J., Kamarck, T., \& Hoberman, H. M. (1985). Measuring the functional components of social support. In I. G. Sarason, \& B. Sarason (Eds.), Social support: Theory, research and applications. (pp. 73-94). The Hague, Holland: Martines Niijhoff.

Cohen, S., \& Syme, L. (1985). Social support and health. London: Academic Press.

Cohen, S., Underwood, L. G., \& Gottlieb, B. H. (Eds.). (2000). Social support measurement and intervention. New York: Oxford.

Cohen, S., \& Wills, T. A. (1985). Stress, social support, and the buffering hypothesis. Psychological Bulletin, 98(2), 310-357.

Collins, D., Baum, A., \& Singer, J. (1983). Coping with chronic stress at three mile island: Psychological and biochemical evidence. Health Psychology, 2(2), 149-166.

Comrey, A. L. (1978). Common methodological problems in factor analytic studies. Journal of Consulting and Clinical Psychology, 46(4), 648-659.

Coolidge, F. L., Segal, D. L., Hook, J. N., \& Stewart, S. (2000). Personality disorders and coping among anxious older adults. Journal of Anxiety Disorders, 14(2), 157-172.

Coyne, J. C., \& Downey, G. (1991). Social factors and psychopathology: Stress, social support, and coping processes. Annual Review of Psychology, 42, 401-425.

Coyne, J. C., Aldwin, C., \& Lazarus, R. S. (1981). Depression and coping in stressful episodes. Journal of Abnormal Psychology, 90(5), 439-447. 
Cronkite, R., Moos, R. H., Twohey, J., Cohen, C., \& Swindle, R., Jr. (1998). Life circumstances and personal resources as predictors of the ten-year course of depression. American Journal of Community Psychology, 26(2), 255-280.

Cuijpers, P., \& Smit, F. (2002). Excess mortality in depression: A meta-analysis of community studies. Journal of Affective Disorders, 72, 227-236.

Cutrona, C. E. (1990). Stress and social support - in search of optimal matching. Journal of Social \& Clinical Psychology, 9, 3-14.

DeLongis, A., \& Holtzman, S. (2005). Coping in context: The role of stress, social support, and personality in coping. Journal of Personality, 73(6), 1633-1656.

Denollet, J., Martens, E. J., Nyklicek, I., Conraads, V. M., \& de Gelder, B. (2008). Clinical events in coronary patients who report low distress: Adverse effect of repressive coping. Health Psychology, 27(3), 302-308.

Dew, M. A., Roth, L. H., Schulberg, H. C., Simmons, R. G., Kormos, R. L., Trzepacz, P. T., \& Griffith, B. P. (1996). Prevalence and predictors of depression and anxiety-related disorders during the year after heart transplantation. General Hospital Psychiatry, 18(Supplement 6), 48-61.

Ding, L., Velicer, W. F., \& Harlow, L. L. (1995). Effects of estimation methods, number of indicators per factor, and improper solutions on structural equation modeling fit indices. Structural Equation Modeling, 2, 119-143.

DiStefano, C. (2002). The impact of categorization with confirmatory factory analysis. Structural Equation Modeling, 9, 327-346.

Doehrman, S. R. (1977). Psychosocial aspects of recovery from coronary heart disease: A review. Social Science \& Medicine, 11, 199-218.

Doering, L. V., Dracup, K., Caldwell, M. A., Moser, D. K., Erickson, V. S., Fonarow, G., \& Hamilton, M. (2004). Is coping style linked to emotional states in heart failure patients? Journal of Cardiac Failure, 10(4), 344-349.

Dozois, D. J. A., Dobson, K. S., \& Ahnberg, J. L. (1998). A psychometric evaluation of the beck depression Inventory-II. Psychological Assessment, 10(2), 83-89.

Dunkel-Schetter, C. (1987). Correlates of social support receipt. Journal of Personality and Social Psychology, 53, 71-80. 
Dunkel-Schetter, C., \& Bennett, T. L. (1990). Differentiating the cognitive and behavioral aspects of social support. In B. Sarason, I. Sarason \& G. Pierce (Eds.), Social support: An interactional view. (pp. 267-296). New York: Wiley.

Edwards, J. R., \& O'Neill, R. M. (1998). The construct validity of scores on the ways of coping questionnaire: Confirmatory analysis of alternative factor structures. Educational and Psychological Measurement, 58(6), 955-983.

Engelfriet, P., Boersma, E., Oechslin, E., Tijssen, J., Gatzoulis, M. A., Thilen, U., . . Mulder, A. (2005). The spectrum of adult congenital heart disease in Europe: Morbidity and mortality in a 5 year follow-up period. European Heart Journal, 26, 2325-2333.

Fan, Y., \& Shi, J. (2009). The challenge-and-response model of normal anxiety. Journal of Adult Development, 16(2), 66-75.

Fauerbach, J. A., Heinberg, L. J., Lawrence, J. W., Bryant, A. G., Richter, L., \& Spence, R. J. (2002). Coping with body image changes following a disfiguring burn injury. Health Psychology, 21(2), 115-121.

Felton, B. J., \& Revenson, T. A. (1984). Coping with chronic illness: A study of illness controllability and the influence of coping strategies on psychological adjustment. Journal of Consulting and Clinical Psychology, 52(3), 343-353.

Finny, S. J., \& DiStefano, C. (2006). Non-normal and categorical data in structural equation modeling. In G. R. Hancock, \& R. O. Mueller (Eds.), Structural equation modeling: A second course. (pp. 269-314). Greenwich, CT: Information Age.

Fisher, J. D., Nadler, A., \& Whitcher-Alagna, S. (1982). Recipient reactions to aid. Psychological Bulletin, 91(1), 27-54.

Folkman, S., \& Lazarus, R. S. (1984). Stress, appraisal, and coping. New York: Springer Publishing Company.

Folkman, S., \& Lazarus, R. S. (1988). Manual for the ways of coping questionnaire. Palo Alto: CA: Consulting Psychologists Press.

Folkman, S., Lazarus, R. S., Dunkel-Schetter, C., DeLongis, A., \& Gruen, R. (1986). The dynamics of a stressful encounter: cognitive appraisal, coping, and encounter outcomes. Journal of Personality and Social Psychology, 50, 992-1003. 
Folkman, S., \& Moskowitz, J. T. (2000). Positive affect and the other side of coping. American Psychologist, 55(6), 647-654.

Folkman, S. (1984). Personal control and stress and coping processes: A theoretical analysis. Journal of Personality and Social Psychology, 46(4), 839-852.

Folkman, S., \& Lazarus, R. S. (1985). If it changes it must be a process: Study of emotion and coping during three stages of a college examination. Journal of Personality and Social Psychology, 48(1), 150-170.

Folkman, S., \& Lazarus, R. S. (1988). Coping as a mediator of emotion. Journal of Personality and Social Psychology, 54(3), 466-475.

Folkman, S., Lazarus, R. S., Dunkel-Schetter, C., DeLongis, A., \& Gruen, R. J. (1986). Dynamics of a stressful encounter: Cognitive appraisal, coping, and encounter outcomes. Journal of Personality and Social Psychology, 50(5), 992-1003.

Forrester, A. W., Lipsey, J. R., Teitelbaum, M. L., DePaulo, J. R., Andrzewski, P. L., \& Robinson, R. G. (1992). Depression following myocardial infarction. International Journal of Psychiatry in Medicine, 22, 33-46.

Forsythe, C. J., \& Compas, B. (1987). Interaction of cognitive appraisals of stressful events and coping. Cognitive Behavior Therapy, 11, 473-485.

Frasure-Smith, N., \& Lesperance, F. (2005). Reflections on depression as a cardiac risk facator. Psychosomatic Medicine, 67(Suppl 1), S19-S25.

Frasure-Smith, N., Lesperance, F., Gravel, G., Masson, A., Juneau, M., Talajic, M., \& Bourassa, M. G. (2000). Social support, depression, and mortality during the first year after myocardial infarction. Circulation, 101(16), 1919-1924.

Frunchak, V. (1989). Gender differences and similarities in cardiac symptoms, psychological distress, and expressed concerns after an episode of acute cardiac ischemia. (Unpublished Masters of Nursing). McGill University, Montreal.

Furlanetto, L. M., Mendlowicz, M. V., \& Romildo Bueno, J. (2005). The validity of the beck depression inventory-short form as a screening and diagnostic instrument for moderate and severe depression in medical inpatients. Journal of Affective Disorders, 86(1), 87-91.

Gantt, L. T. (1992). Growing up heartsick: The experiences of young women with congenital heart disease. Health Care for Women International, 13(3), 241-248. 
Gardner, F. V., \& Angelini, G. D. (1995). Psychological aspects of congenital heart disease. Cardiology in the Young, 5(4), 302-309.

Garson, J., A., Williams, J., R. B., \& Reckless, J. (1974). Long-term follow-up of patients with tetralogy of fallot: Physical health and psychopathology. The Journal of Pediatrics, 85(3), 429-433.

Garver, M. S., \& Mentzer, J. T. (1999). Logistic research methods: Employing structural equation modeling to test for construct validity. Journal of Business Logistics, 20(1), 3357.

Goossens, P. J. J., Knoppert-van der Klein, E. A. M., \& van Achterberg, T. (2008). Coping styles of outpaitents with a bipolar disorder. Archives of Psychiatric Nursing, 22(5), 245-253.

Green, A. (2004). Outcomes of congenital heart disease. Pediatric Nursing, 30(4), 280-284.

Green, D. L., \& Pomeroy, E. C. (2007). Crime victimization: assessing differences between violent and non-violent experiences. Victims and Offenders, 2(1), 63-76.

Gupta, S., Giuffre, R. M., Crawford, S., \& Waters, J. (1998). Covert fears, anxiety and depression in congenital heart disease. Cardiology in the Young, 8(4), 491-499.

Haber, M. G., Cohen, J. L., Lucas, T., \& Baltes, B. B. (2007). The relationship between selfreported received and perceived social support: a meta-analytic review. American Journal of Community Psychology, 39(1-2), 133-144.

Hann, D., Baker, F., Denniston, M., Gesme, D., Reding, D., Flynn, T., . . Kieltyka, L. (2002). The influence of social support on depressive symptoms in cancer patients : Age and gender differences. Journal of Psychosomatic Research, 52(5), 279-283.

Harris, C. A., \& D'Eon, J. L. (2008). Psychometric properties of the beck depression inventory-second edition (BDI-II) in individuals with chronic pain. Pain, 137(3), 609622.

Hart, E. M., \& Garson, A. J. (1993). Psychosocial concerns of adults with congenital heart disease. Cardiology Clinics, 11(4), 711-715.

Helgeson, V. S. (2003). Social support and quality of life. Quality of Life Research, 12 (Suppl 1), 25-31. 
Hill, L., \& Kennedy, P. (2002). The role of coping strategies in mediating subjective disability in people who have psoriasis. Psychology, Health \& Medicine, 7(3), 261-269.

Hoelter, D. R. (1983). The analysis of covariance structure: Goodness-of-fit indices. Sociological Methods and Research, 11, 325-344.

Hoffman, J. I. E., \& Kaplan, S. (2002). The incidence of congenital heart disease. Journal of the American College of Cardiology, 39(12), 1890-1900.

Holahan, C. J., \& Moos, R. H. (1987). Personal and contextual determinants of coping strategies. Journal of Personality and Social Psychology, 52(5), 946-955.

Holahan, C. J., \& Moos, R. H. (1981). Social support and psychological distress: A longitudinal analysis. Journal of Abnormal Psychology, 90(4), 365-370.

Holahan, C. J., \& Moos, R. H. (1987). Personal and contextual determinants of coping strategies. Journal of Personality and Social Psychology, 52(5), 946-955.

Holahan, C. J., Moos, R. H., Holahan, C. K., \& Brennan, P. L. (1995). Social support, coping, and depressive symptoms in a late-middle-aged sample of patients reporting cardiac illness. Health Psychology, 14(2), 152-163.

Holahan, C. J., Moos, R. H., Holahan, C. K., \& Brennan, P. L. (1997). Social context, coping strategies, and depressive symptoms: An expanded model with cardiac patients. Journal of Personality and Social Psychology, 72(4), 918-928.

Holtzman, S., Newth, S., \& DeLongis, A. (2004). The role of social support in coping with daily pain among patients with rheumatoid arthritis. Journal of Health Psychology, 9(5), 677-695.

Horner, T., Lieberthson, R., \& Jellinek, M. S. (2000). Psychosocial profile of adults with complex congenital heart disease. Mayo Clinic Proceedings, 75, 31-36.

House, J. S. (1981). Work, stress and social support. MA: Addison-Wesley.

Hu, L., \& Bentler, P. M. (1999). Cutoff criteria for fit indexes in covariance structure analysis: Conventional criteria versus new alternatives. Structural Equation Modeling, 6, $1-55$.

Hurley, A. E., Scandura, T. A., Schriesheim, C. A., Brannnick, M. T., Seers, A., Vandenbertn, R. J., \& Williams, L. J. (1997). Exploratory and confirmatory factor 
analysis: Guidelines, issues, and alternatives. Journal of Organizational Behavior, 18, 667-683.

Jarrett, R. B. (1990). Psychosocial aspects of depression and the role of psychotherapy. Journal of Clinical Psychiatry, 51 (6 (suppl)), 26-35.

Jefferies, J. L., Noonan, J. A., Keller, B. B., Wison, J. F., \& Griffith, C., III. (2004). Quality of life and social outcomes in adults with congenital heart disease living in rural areas of kentucky. The American Journal of Cardiology, 94, 263-266.

Jones, D. J., Bromberger, J. T., Sutton-Tyrrell, K., \& Matthews, K. A. (2003). Lifetime history of depression and carotid atherosclerosis in middle-aged women. Archives of General Psychiatry, 60, 153-160.

Joreskog, K., \& Sorbom, D. (1993). LISREL 8: Structural equation modeling with the simplis command language. Lincolnwood, IL: Scientific Software International, Inc.

Joreskog, K., \& Sorbom, D. (1993). LISREL 8: User's reference guide. Lincolnwood, IL: Scientific Software International, Inc.

Kaba, E., Thompson, D. R., \& Burnard, P. (2000). Coping after heart transplantation: A descriptive study of heart transplant recipients' methods of coping. Journal of Advanced Nursing, 32(4), 930-936.

Kenny, D. A. (2011). Measuring model fit. Retrieved 30/03, 2012, from http://davidakenny.net/cm/fit.htm

Kessler, R. C. (1992). Perceived support and adjustment to stress: Methodological considerations. In H. O. F. Veiel, \& U. Baumann (Eds.), The meaning and measurement of social support (pp. 273-289). New York: Hemisphere Publishing Corporation.

Kessler, R. C., \& McLeod, J. D. (1985). Social support and mental health in community samples. New York: Academic Press.

Kim, J., Han, J., Shaw, B., McTavish, F., \& Gustafson, D. (2010). The roles of social support and coping strategies in predicting breast cancer patients' emotional well-being. Journal of Health Psychology, 15(4), 543-552.

King, K. B., Reis, H. T., Porter, L. A., \& Norsen, L. H. (1993). Social support and long-term recovery from coronary artery surgery: Effects on patients and spouses. Health Psychology, 12(1), 56-63. 
Kinsinger, S. W., McGregor, B. A., \& Bowen, D. J. (2009). Perceived breast cancer risk, social support, and distress among a community-based sample of women. Journal of Psychosocial Oncology, 27(2), 230-247.

Kokkonen, J., \& Paavilainen, T. (1992). Social adaptation of young adults with congenital heart disease. International Journal of Cardiology, 36(1), 23-29.

Kovacs, A. H., Saidi, A. S., Kuhl, E. A., Sears, S. F., Silversides, C., Harrison, J. L., . . . Nolan, R. P. (2009). Depression and anxiety in adult congenital heart disease: Predictors and prevalence. International Journal of Cardiology, 137(2), 158-164.

Kovacs, A. H., Sears, S. F., \& Saidi, A. (2005). Biopsychosocial experiences of adults with congenital heart disease: Review of the literature. American Heart Journal, 150(2), 193201.

Kovacs, A. H., Silversides, C., Saidi, A., \& Sears, S. F. (2006). The role of the psychologist in adult congenital heart disease. Cardiology Clinics, 24, 607-618.

Lakey, B., \& Cassady, P. B. (1990). Cognitive processes in perceived social support. Journal of Personality and Social Psychology, 59, 337-343.

Lakey, B., \& Cohen, S. (2000). Social support theory and measurement. In S. Cohen, L. G. Underwood \& B. H. Gottlieb (Eds.), Social support measurement and intervention (pp. 29-52). New York: Oxford University Press.

Lakey, B., Drew, J. B., Anan, R. M., Sirl, K., \& Butler, C. (2004). Negative interpretations of interpersonal situations and the relation between low perceived support and psychological distress among divorced adults. Journal of Applied Social Psychology, 34(5), 1030-1047.

Lapore, S. J., Glaser, D. B., \& Roberts, K. J. (2008). On the positive relation between received social support and negative effect: A test of the triage and self-esteem threat models in women with breast cancer. Psycho-Oncology, 17, 1210-1215.

Lazarus, R. S. (1966). Psychological stress and the coping process. New York: McGraw-Hill.

Lazarus, R. S., \& Folkman, S. (1984). Stress, appraisal, and coping. New York: Springer Publishing. 
Levine, J., Warrenburg, S., Kerns, R., Schwartz, G., Delaney, R., Fontana, A., . . Cascione, R. (1987). The role of denial in recovery from coronary heart disease. Psychosomatic Medicine, 49(2), 109-117.

Leyfer, O. T., Ruberg, J. L., \& Woodruff-Borden, J. (2006). Examination of the utility of the beck anxiety inventory and its factors as a screener for anxity disorders. Anxiety Disorders, 20, 444-458.

Linden, W., \& Vodermaier, A. (2012). Mismatch of desired versus perceived social support and associated levels of anxiety and depression in newly diagnosed cancer patients. Support Cancer Care, 20, 1449-1456.

Lindsey, A. M., \& Yates, B. C. (2004). Social support: Conceptualization and measurement instruments. In M. Frank-Stromborg, \& S. J. Olsen (Eds.), Instruments for clinical health-care research (pp. 164-199). Sudbury, MA: Jones and Bartlett Publishers.

Lip, G. Y. H., Lane, D. A., \& Millane, T. A. (2008). Psychological interventions for depression in adolescent and adult congenital heart disease (review). Cochrane Database of Systematic Reviews, (3), 1-9.

Livecchi, T. A. (2004). Psychosocial issues affecting adults with congenital heart disease: One patient's perspective. Nursing Clinics of North America, 39, 787-791.

Lundqvist, L., \& Ahlström, G. (2006). Psychometric evaluation of the ways of coping questionnaire as applied to clinical and nonclinical groups. Journal of Psychosomatic Research, 60(5), 485-493.

Lyon, M. E., Kuehl, K., \& McCarter, R. (2006). Transition to adulthood in congenital heart disease: Missed adolescent milestones. Journal of Adolescent Health, 39, 121-124.

Lyyra, T. M., \& Heikkinen, R. L. (2006). Perceived social support and mortality in older people. Journal of Gerontology, Series B, 61(3), S147-152.

Maisel, N. C., \& Gable, S. L. (2009). The paradox of received social support. Psychological Science, 20(8), 928-932.

Manne, S. L., Pape, S. J., Taylor, K. L., \& Dougherty, J. (1999). Spouse support, coping, and mood among individuals with cancer. Annals of Behavioral Medicine, 21(2), 111-121. 
Manne, S. L., \& Zautra, A. J. (1989). Spouse criticism and support: Their association with coping and psychological adjustment among women with rheumatoid arthritis. Journal of Personality and Social Psychology, 56(4), 608-617.

Markstrom, C. A., Marshall, S. K., \& Tryon, R. J. (2000). Resiliency, social support, and coping in rural low-income appalachian adolescents from two racial groups. Journal of Adolescence, 23(6), 693-703.

Marsac, M. L., Funk, J. B., \& Nelson, L. (2006). Coping styles, psychological functioning and quality of life in children with asthma. Child: Care, Health and Development, 33(4), 360-367.

Martucci, M., Balestrieri, M., \& Bisoffi, G. (1999). Evaluating psychiatric morbidity in a general hospital: A two-phase epidemiological survey. Psychological Medicine, 29, 823832.

Marzari, C., Maggi, S., Manzato, E., Destro, C., Noale, M., Bianchi, D., . . the Italian Longitudinal Study on Aging Working Group,. (2005). Depressive symptoms and development of coronary heart disease events: The Italian longitudinal study on aging. Journals of Gerontology Series A: Biological Sciences and Medical Sciences, 60(1), 8592.

Masi, G., \& Brovedani, P. (1999). Adolescents with congenital heart disease: Psychopathological implications. Adolescence, 34(133), 185-191.

McCabe, M. P., McKern, S., \& McDonald, E. (2004). Coping and psychological adjustment among people with multiple sclerosis. Journal of Psychosomatic Research, 56(3), 355361.

McColl, M. A., \& Skinner, H. (1995). Assessing inter- and intrapersonal resources: Social support and coping among adults with a disability. Disability and Rehabilitation: An International, Multidisciplinary Journal, 17(1), 24-34.

McMurray, R., Kendall, L., Parsons, J. M., Quirk, J., Veldtman, G. R., Lewin, R. J. P., \& Sloper, P. (2001). A life less ordinary: Growing up and coping with congenital heart disease. Coronary Health Care, 5, 51-57.

Mendes de Leon, C. F., Czajkowski, S. M., Freedland, K. E., Bang, H., Powell, L. H., Wu, C., . . Blumenthal, J. A. (2006). The effect of a psychosocial intervention and quality 
of life after acute myocardial infarction: The enhancing recovery in coronary heart disease (ENRICHD) clinical trial. Journal of Cardiopulmonary Rehabilitation, 26(1), 913.

Mirowsky, J., \& Ross, C. E. (2003). Education, social status and health. New York: Aldine de Gruyter.

Moons, P., Van Deyk, K., De Bleser, L., Marquet, K., Raes, E., De Geest, S., \& Budts, W. (2006). Quality of life and health status in adults with congenital heart disease: A direct comparison with healthy counterparts. European Journal of Cardiovascular Prevention and Rehabilitation, 13(3), 407-413.

Moser, D. K., \& Dracup, K. (1995). Psychosocial recovery from a cardiac event: The influence of perceived control. Heart \& Lung, 24(4), 273-280.

Munet-Vilaro, F., Gregorich, S. E., \& Folkman, S. (2002). Factor structure of the Spanish version of the ways of coping questionnaire. Journal of Applied Social Psychology, 32(9), 1938-1954.

Muthen, L. K., \& Muthen, B. O. (2006). Mplus user's guide. (4th ed.). Los Angeles: Muthen $\&$ Muthen.

Muthen, L. K., \& Muthen, B. O. (2010). Mplus statistical analysis with latent variables: User's guide. (6th ed.). Los Angeles, CA: Muthen \& Muthen.

Myaskovsky, L., Amanda Dew, M., Switzer, G. E., Hall, M., Kormos, R. L., Coycoolea, J. M., .. . McCurry, K. R. (2003). Avoidant coping with health problems is related to poorer quality of life among lung transplant candidates. Progress in Transplantation, 13(3), 183-192.

Namir, S., Wolcott, D. L., Fawzy, F. I., \& Alumbaugh, M. J. (1987). Coping with AIDS: Psychological and health implications. Journal of Applied Social Psychology, 17(3), 309-328.

Niwa, K., Tateno, S., Tatebe, S., Fujita, K., Sugita, K., Terai, M., . . Takahashi, O. (2002). Social concern and independence in adults with congenital heart disease. Journal of Cardiology, 39(5), 259-266 
Norris, F. H., \& Kaniasty, K. (1996). Received and perceived social support in times of stress: A test of the social support deterioration deterrence model. Journal of Personality and Social Psychology, 71(3), 498-511.

Nunnally, J. C. (1978). Psychometric theory. New York: McGraw-Hill.

Osman, A., Barrios, F. X., Aukes, D., Osman, J. R., \& Markway, K. (1993). The beck anxiety inventory: Psychometric properties in a community population. Journal of Psychopathology and Behavioral Assessment, 15, 287-297.

Osman, A., Downs, W. R., Barrios, F. X., Kopper, B. A., Gutierrez, P. M., \& Chiros, C. E. (1997). Factor structure and psychometric characteristics of the beck depression inventory-II. Journal of Psychopathology and Behavioral Assessment, 19(4), 359-376.

Osman, A., Hoffman, J., Barrios, F. X., Kopper, B. A., Breitenstein, J. L., \& Hahn, S. K. (2002). Factor structure, reliability, and validity of the beck anxiety inventory in adolescent psychiatric inpatients. Journal of Clinical Psychology, 58(4), 443-456.

Osman, A., Kopper, B. A., Barrios, F. X., Osman, J. R., \& Wade, T. (1997). The beck anxiety inventory: Reexamination of factor structure and psychometric properties. Journal of Clinical Psychology, 53(1), 7-14.

Oxman, T. E., Freeman Jr., D. H., Manheimer, E. D., \& Stukel, T. (1994). Social support and depression after cardiac surgery in elderly patients. The American Journal of Geriatric Psychiatry, 2(4), 309-323.

Parker, J. D., \& Endler, N. S. (1992). Coping with coping assessment: a critical review. European Journal of Personality, 6(5), 321-344.

Parker, J. D., Endler, N. S., \& Bagby, R. M. (1993). If it changes, it might be unstable: Examining the factor structure of the ways of coping questionnaire. Psychological Assessment, 5(3), 361-368-368.

Parkes, K. R. (1986). Coping in stressful episodes: The role of individual differences, environmental factors, and situational characteristics. Journal of Personality and Social Psychology, 51(6), 1277-1292.

Peacock, E. J., Wong, P. T., \& Reker, G. T. (1993). Relations between appraisals and coping schemas: Support for the congruence model. Canadian Journal of Behavioural Science/Revue Canadienne Des Sciences Du Comportement, 25(1), 64-80. 
Penninx, B. W. J. H., van Tilburg, T., Boeke, A. J., D. J.H., Kriegsman, D. M. W., \& van Eijk, J. T. M. (1998). Effects of social support and personal coping resources on depressive symptoms: Different for various chronic diseases? Health Psychology, 17(6), 551-558.

Perez, J. E., Chartier, M., Koopman, C., Vosvick, M., Gore-Felton, C., \& Spiegel, D. (2009). Spiritual striving, acceptance coping, and depressive symptoms among adults living with HIV/AIDS. Journal of Health Psychology, 14(1), 88-97.

Peters, K. F., Apse, K. A., Blackford, A., McHugh, B., Michalic, D., \& Biesecker, B. B. (2005). Living with Marfan syndrome: Coping with stigma. Clinical Gerontology, 68, 614.

Plumb, J. C., Orsillo, S. M., \& Luterek, J. A. (2004). A preliminary test of the role of experiential avoidance in post-event functioning. Journal of Behavior Therapy and Experimental Psychiatry, 35(3), 245-257.

Popelova, J., Slavik, Z., \& Skovranek, J. (2001). Are cyanosed adults with congenital cardiac malformations depressed? Cardiology in the Young, 11, 379-384.

Purden, M. A. (1995). Wives' marital role and psychosocial adjustment: A study of patient and spouse outcomes two months after a myocardial infarction. (Unpublished $\mathrm{PhD}$ Dissertation). McGill University, Montreal.

Richaud de Minzi, M. C., \& Sacchi, C. (2001). Effect of inapplicable items in the factor structure of the Spanish version of the ways of coping questionnaire. Psychological Reports, 88, 115-122.

Richter, P., Werner, J., Heerlein, A., \& Kraus, H. S. (1998). On the validity of the beck depression inventory. Psychopathology, 31, 160-168.

Ridner, S. H. (2004). Psychological distress: Concept analysis. Journal of Advanced Nursing, 45(5), 536-545.

Rodrigue, J. R., Boggs, S. R., Weiner, R. S., \& Behen, J. M. (1993). Mood, coping style, and personality functioning among adult bone marrow transplant candidates.

Psychosomatics, 34(2), 159-165. 
Roesch, S. C., Adams, L., Hines, A., Palmores, A., Vyas, P., Tran, C., . . Vaughn, A. (2005). Coping with prostate cancer: A meta-analytic review. Journal of Behavioral Medicine, 28(3), 281-293.

Rook, K. S. (1984). The negative side of social interaction: Impact on psychological wellbeing. Journal of Personality and Social Psychology, 46(5), 1097-1108.

Rosengren, A., Hawken, S., \& Ounpuu, S., . . Yusuf, S. (2004). Association of psychosocial risk factors with risk of acute myocardial infarction in 11119 cases and 13648 controls from 52 countries (the INTERHEART study): case-control study. Lancet, 364, 953-962.

Scherer, R. F., Wiebe, F. A., Luther, D. C., \& Adams, J. S. (1988). Dimensionality of coping: Factor stability using the ways of coping questionnaire. Psychological Reports, 62(3), 763-770.

Shnek, Z. M., Irvine, J., Stewart, D., \& Abbey, S. (2001). Psychological factors and depressive symptoms in ischemic heart disease. Health Psychology, 20(2), 141-145.

Schroder, K., Schwarzer, R., \& Endler, N. S. (1997). Predicting cardiac patients' quality of life from the characteristics of their spouses. Journal of Health Psychology, 2(2), 231244.

Schroder, K., Schwarzer, R., \& Konertz, W. (1998). Coping as a mediator in recovery from cardiac surgery. Psychology and Health, 13, 83-97.

Schulz, U., \& Schwarzer, R. (2004). Long-term effects of spousal support on coping with cancer after surgery. Journal of Social \& Clinical Psychology, 23, 716-732.

Schulz, R., \& Decker, S. (1985). Long-term adjustment to physical disability: The role of social support, perceived control, and self-blame. Journal of Personality and Social Psychology, 48(5), 1162-1172.

Schumacker, R. E., \& Lomax, R. G. (2004). A beginner's guide to structural equation modeling (2nd ed.). Mahwah, NJ: Lawrence Erlbaum Associates.

Schuster, T. L., Kessler, R. C., \& Aseltine Jr., R. H. (1990). Supportive interactions, negative interactions, and depressed mood. American Journal of Community Psychology, 18(3), 423-438.

Schwarzer, R., \& Schulz, U. Berlin social support scales (BSSS). Retrieved 06, 2009, from http://userpage.fu-berlin.de/ health/bsssdocument.pdf 
Schwarzer, R., \& Schwarzer, C. (1996). A critical survey of coping instruments. In M. Zeidner, \& N. S. Endler (Eds.), Handbook of coping: Theory, research and applications. (pp. 107-132). New York: Wiley.

Shen, B., Myers, H. F., \& McCreary, C. P. (2006). Psychosocial predictors of cardiac rehabilitation quality-of-life outcomes. Journal of Psychosomatic Research, 60(1), 3-11.

Siegal, B. R., Calsyn, R. J., \& Cuddihee, R. M. (1987). The relationship of social support to psychological adjustment in end-stage renal disease patients. Journal of Chronic Diseases, 40(4), 337-344.

Simon, A. E., \& Palmer, S. C. Coyne, J. C. (2007). Cancer and depression. In A. Steptoe (Ed.), Depression and physical illness (pp. 211-237). New York: Cambridge University Press.

Smith, C. A., \& Wallston, K. A. (1992). Adaptation in patients with chronic rheumatoid arthritis: Application of a general model. Health Psychology, 11(3), 151-162.

Sorlie, T., \& Sexton, H. C. (2001). The factor structure of "the ways of coping questionnaire" and the process of coping in surgical patients. Personality and Individual Differences, 30, 961-975.

Spielberger, C. D. (1972). Anxiety: Current trends in theory and research. New York, N. Y.: Academic Press.

Spurkland, I., Bjornstad, P. G., Lindberg, H., \& Seem, E. (1993). Mental health and psychosocial functioning in adolescents with congenital heart disease. A comparison between adolescents born with severe heart defect and atrial septal defect. Acta Paediatrics, 82, 71-76.

Stansfeld, S. A., Fuhrer, R., \& Shipley, M. J. (1998). Types of social support as predictors of psychiatric morbidity in a cohort of British civil servants (whitehall II study). Psychological Medicine, 28, 881-892.

Stanton, A. L., \& Snider, P. R. (1993). Coping with a breast cancer diagnosis: A prospective study. Health Psychology, 12(1), 16-23.

Steer, R. A., Ball, R., Ranieri, W. F., \& Beck, A. T. (1999). Dimensions of the beck depression inventory-II in clinically depressed outpatients. Journal of Clinical Psychology, 55(1), 117-128. 
Steer, R. A., \& Clark, D. A. (1997). Psychometric characteristics of the back depression inventory-II with college students. Measurement \& Evaluation in Counseling \& Development (American Counseling Association), 30(3), 128-143.

Steer, R. A., \& Ball, R. (1999). Dimensions of the beck depression inventory-II in clinically depressed outpatients. Journal of Clinical Psychology, 55(1), 117-128.

Steer, R. A., Ranieri, W. F., Beck, A. T., \& Clark, D. A. (1993). Further evidence for the validity of the beck anxiety inventory with psychiatric outpatients. Journal of Anxiety Disorders, 7(3), 195-205.

Steer, R. A., Rissmiller, D. J., Ranieri, W. F., \& Beck, A. T. (1993). Structure of the computer-assisted beck anxiety inventory with psychiatric inpatients. Journal of Personality Assessment, 60(3), 532.

Steptoe, A. (Ed.). (2007). Depression and physical illness. Cambridge: Cambridge University Press.

Stewart, M. J. (1989). Social support instruments created by nurse investigators. Nursing Research, 38(5), 268-275.

Suls, J., \& Fletcher, B. (1985). The relative efficacy of avoidant and nonavoidant coping strategies: A meta-analysis. Health Psychology, 4(3), 249-288.

Tabachnick, B. G., \& Fidell, L. S. (2007). Using multivariate statistics. (5th ed.). Boston: Allyn and Bacon.

Tamres, L. K., Janicki, D., \& Helgeson, V. S. (2002). Sex differences in coping behavior: A meta-analytic review and an examination of relative coping. Personality and Social Psychology Review, 6(1), 2-30.

Taussig, H. B. (2011). Congenital heart disease: Overview of adult CHD. Retrieved 04/26, 2012, from http://www.pted.org/?id=overview1

Tennen, H., \& Herzberger, S. (1985). Ways of coping scale. In D. J. Keyser, \& R. C. Sweetland (Eds.), Test critiques (Vol. 3, pp. 686-697). Kansas City, MO: Test Corporation of America.

Thoits, P. A. (1995). Stress, coping, and social support processes: Where are we? what next? Journal of Health \& Social Behavior, 36, 53-79. 
Thomason, B. N. (1997). The lived experience of adolescents with significant congenital heart disease. (Unpublished $\mathrm{PhD}$ ), Louisiana State University, Louisiana.

Tsay, S., \& Chao, Y. C. (2002). Effects of perceived self-efficacy and functional status on depression in patients with chronic heart failure. Journal of Nursing Research, 10(4), 271-277.

Turner, R. J. (1983). Direct, indirect and moderating effects of social support upon psychological distress and associated conditions. In H. B. Kaplan (Ed.), (pp. 105-155). New York: Academic Press.

Uchino, B. N. (2004). Social support and physical health: Understanding the health consequences of relationships. New Haven: Yale University Press.

Utens, E. M. W. J., Verhulst, F. C., Erdman, R. A. M., Meijboom, F. J., Duivenvoorden, H. J., Bos, E., . . Hess, J. (1994). Psychosocial funtioning of young adults after surgical correction for congenital heart disease in childhood: A follow-up study. Journal of Psychosomatic Research, 38(7), 745-758.

Utens, E. M., Versluis-Den Bieman, H. J., Verhulst, F. C., Meijboom, F. J., Erdman, R. A., \& Hess, J. (1998). Psychopathology in young adults with congenital heart disease. European Heart Journal, 19, 647-651.

Uzark, K. (1992). Counseling adolescents with congenital heart disease. Journal of Cardiovascular Nursing, 6(3), 65-73.

Uzark, K., Jones, K., Slusher, J., Limbers, C. A., Burwinkle, T. M., \& Varni, J. W. (2008). Quality of life in children with heart disease as perceived by children and parents. Pediatrics, 121(5), e1060-e1067.

Valentiner, D. P., Holahan, C. J., \& Moos, R. H. (1994). Social support, appraisals of event controllability, and coping: An integrative model. Journal of Personality and Social Psychology, 66(6), 1094-1102.

Veiel, H. O. F., Kuhner, C., Brill, G., \& Ihle, W. (1992). Grown-up survivors of congenital heart disease: Who knows? who cares? British Journal of Sports Medicine, 26(3), 139142.

Verstappen, A., Pearson, D., \& Kovacs, A. H. (2006). Adult congenital heart disease: The patient's perspective. Cardiology Clinics, 24, 515-529. 
Viljoen, J. L., Iverson, G. L., Griffiths, S., \& Woodward, T. S. (2003). Factor structure of the beck depression inventory-II in a medical outpatient sample. Journal of Clinical Psychology in Medical Settings, 10(4), 289-291.

Vollman, M. W., LaMontagne, L. L., \& Hepworth, J. T. (2007). Coping with depressive symptoms in adults living with heart failure. Journal of Cardiovascular Nursing, 22(2), 125-130.

Waller, N. G. (1989). The effect of inapplicable item responses on the structure of behavioral checklist data: A cautionary note. Multivariate Behavioral Research, 24(1), 125.

Ward, L. C. (2006). Comparison of factor structure models for the beck depression inventory--II. Psychological Assessment, 18(1), 81-88.

Ware, W. B., \& Benson, J. (1975). Appropriate statistics and measurement scales. Science Education, 59, 575-582.

Ware Jr., J. E., Kosinski, M., Bjorner, J. B., Turner-Bowker, D. M., Gandek, B., \& Maruish, M. E. (2007). User's manual for the SF-36v2TM health survey (2nd ed). Lincoln, RI.: Quality Metric Incorporated.

Ware Jr., J. E., Kosinski, M., Gandek, B., Aaronson, N. K., Apolone, G., Bech, P., . . Sullivan, M. (1998). The factor structure of the SF-36 health survey in 10 countries: Results from the IQOLA project. Journal of Clinical Epidemiology, 51(11), 1159-1165.

Warnes, C. A. (2005). The adult with congenital heart disease: Born to be bad? Journal of American College of Cardiology, 46(1), 1-8.

Warnes, C. A., Liberthson, R., Danielson, G. K., Dore, A., Harris, L., Hoffman, J. I. E., .. . Webb, G. D. (2001). Task force 1: The changing profile of congenital heart disease in adult life. Journal of the American College of Cardiology, 37(5), 1170-1175.

Wells, K. J., Booth-Jones, M., \& Jacobsen, P. B. (2009). Do coping and social support predict depression and anxiety in patients undergoing hematopoietic stem cell transplantation? Journal of Psychosocial Oncology, 27(3), 297-315.

Wethington, E., \& Kessler, R. C. (1986). Perceived support, received support, and adjustment to stressful life events. Journal of Health and Social Behavior, 27(1), 78-89. 
Whisman, M. A., \& Perez, J. E. (2000). Factor structure of the beck depression inventorysecond edition (BDI-II) in a student sample. Journal of Clinical Psychology, 56(4), 545551.

William, S. L. (1995). Self-efficacy, anxiety, and phobic disorders. In J. E. Maddux (Ed.), Self-efficacy, adatation, and adjustment: Theory, research, and application (). New York: Plenum Press.

Wills, T. A., \& Shinar, O. (2000). Social support theory and measurement. In S. Cohen, L. G. Underwood \& B. H. Gottlieb (Eds.), Social support measurement and intervention (pp. 87-135). New York: Oxford University Press.

Wineman, N. M., Durand, E. J., \& McCulloch, B. J. (1994). Examination of the factor structure of the ways of coping questionnaire with clinical populations. Nursing Research, 43(5), 268-273.

Wortman, C. B. (1984). Social support and the cancer patient. 1984, 53(10 (supplement)), 2339-2360.

Wright, M., Jarvis, S., Wannamaker, E., \& Cook, D. (1985). Congenital heart disease: Functional abilities in young adults. Archives of Physical Medicine and Rehabilitation, 66, 289-293.

Yeh, S. J., \& Chou, H. (2007). Coping strategies and stressors in patients with hemodialysis. Psychosomatic Medicine, 69, 182-190.

Yip, B., Rowlinson, S., \& Oi, L. S. (2008). Coping strategies as moderators in the relationship between role overload and burnout. Construction Management \& Economics, 26(8), 869-880.

Yu, D. S. F., Lee, D. T. F., Woo, J., \& Thompson, D. R. (2004). Correlates of psychological distress in elderly patients with congestive heart failure. Journal of Psychosomatic Research, 57(6), 573-581.

Yusuf, S., Hawken, S., Ounpuu, S., Dans, T., Avezum, A., Lanas, F., McQueen, M., Budaj, A., Pais, P., Varigos, J., \& Lisheng, L. (2004). Effect of potentially modifiable risk factors associated with myocardial infarction in 52 countries (the INTERHEART study): case-control study. Lancet, 364(9438), 937-952. 
Zarit, S., Reever, K., \& Bach-Peterson, J. (1980). Relatives of impaired elderly correlates of feelings of burden. Gerontologist, 20, 649-655.

Zwick, W. R., \& Velicer, W. F. (1986). Comparison of five rules for determining the number of components to retain. Psychological Bulletin, 99(3), 432-442. 
Appendix A

Letter of Invitation

\&

Consent Form 


\section{THE U N IVER I T Y OF B R I T IS H C O L U M B I A}
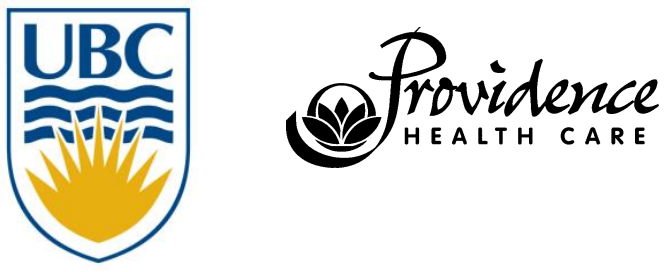

Dr. Joy Johnson (Principal Investigator)

302-6190 Agronomy Road

Vancouver, B.C. Canada V6T 1 Z4

Tel: (604) 827-4020

\section{Relationship between types of social support, coping styles, and psychological distress in individuals living with congenital heart disease}

I am writing to you today to ask you to consider participating in a research study that I am completing as part of my doctoral studies in nursing.

The purpose of this study is to learn more about how adults who are born with congenital heart disease adjust to the strains of everyday life. Recent medical and surgical advancements are now making it possible for people with congenital heart disease to live long and productive lives. To date research has focused on the medical and surgical management of people with congenital heart disease. We know that while many individuals with congenital heart disease appear to maintain fairly well-adjusted lives, others struggle with psychological distress including depression and anxiety. The purpose of this study is to learn more about the factors that influence or contribute to such distress.

If you choose to participate, the information you provide us will be extremely valuable in helping us identify the factors that contribute to the psychological wellbeing in people living with congenital heart disease. We are interested in learning about how you cope with, and manage living with a congenital heart defect. It is our hope that the knowledge we gain from you will not only help us identify those who are struggling with psychological distress, but also assist us in developing strategies to reduce and manage distress. Ultimately, the goal is to help those with congenital heart disease achieve a quality of life that is both meaningful and satisfying.

The study involves answering a set of questionnaires that should take no longer than 30-45 minutes to complete.

I have attached a consent form that further explains the study. Please read it and contact me (778) 848-7947 or my supervisor Dr. Joy Johnson (604) 822-7435 if you have any questions. 
When you attend the clinic for your appointment, you will be asked to sign the informed consent form and you will then be given an envelope with the study questionnaires.

Thank you very much.

Mi-Yeon Kim, MScN, RN, Doctoral Student T201-2211 Wesbrook Mall

Vancouver, B.C. Canada V6T 2B5

Tel: (778) 848-7947 


\section{THE UNIVERSITY OF B RITISH C O L UMB I A}
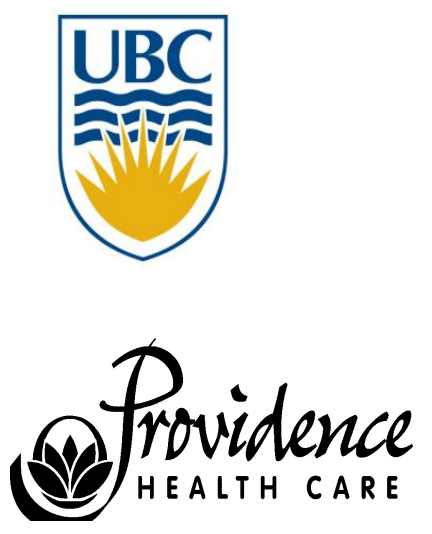

Dr. Joy Johnson (PI)

302-6190 Agronomy Road

Vancouver, B.C. Canada V6T 1Z4

Tel: (604) 827-4020

Mi-Yeon Kim (Co-PI)

T201-2211 Wesbrook Mall

Vancouver, B.C. Canada V6T 2B5

$\mathrm{Tel}:$ (778) 848-7947

\section{CONSENT FORM}

\section{Title of study:}

Relationship between types of social support, coping styles, and psychological distress in individuals living with congenital heart disease

Principal Investigator:

Joy L. Johnson, PhD, Professor, School of Nursing, University of British Columbia

\section{Co-Investigator:}

Mi-Yeon Kim, PhD Candidate, School of Nursing, University of British Columbia

\section{Funding}

The study is part of the requirements for the doctoral degree of the Co-Investigator (M. Kim) and is not funded by any agencies or institutions.

\section{Introduction}

You are being invited to take part in this research study because you were born with congenital heart disease and have the experience of living and adjusting to the strains of everyday life. If you are 19 years and older and were born with congenital heart disease, then you are cordially invited to participate in this study.

\section{Background}

Recent medical and surgical advancements are now making it possible for people with congenital heart disease to live long and productive lives. To date research has focused on the medical and surgical management of people with congenital heart disease, but not on the management of their psychological well-being. We know that while many individuals with congenital heart disease appear to maintain fairly well-adjusted lives, others struggle with psychological distress including depression and anxiety. 


\section{Purpose}

The purpose of this study is to learn about factors that predict the psychological distress in people living with congenital heart disease. More specifically, the investigators of this study would like to study the reason(s) why some people with congenital heart disease suffer from emotional distress such as depression and/or anxiety while others do not.

\section{Study Procedures}

This study will take place at the Adult Congenital Heart Disease Clinic in St. Paul's Hospital, Vancouver, Canada, and a total number of 250-300 volunteer subjects will be enrolled for the study. If you agree to participate in this study, the following will occur:

1. You will be asked to sign the consent form in the presence of the investigator (MiYeon Kim) when you report to the reception desk at the Adult Congenital Heart Disease Program Outpatient Clinic at St. Paul's Hospital.

2. After the consent has been signed, you will be given an envelope containing a set of questionnaires.

3. You will be asked to complete a set of 6 questionnaires (e.g., Beck Depression Inventory, Ways of Coping Questionnaire), and demographic questionnaire (e.g., that measures age, education, etc.).

4. You may complete these questionnaires while you are waiting for your appointments, or whenever it is convenient for you throughout the day. It should take a total of 3045 minutes to complete the questionnaires.

5. When completed, please insert the questionnaires into the envelope provided and seal the envelope.

6. Submit the envelope containing the questionnaires to the reception desk at the Adult Congenital Heart Disease Program Clinic where you first reported for your appointment.

7. You can also take the questionnaires home with you and mail them in. Please use a stamped envelope provided and send it to:

Mi-Yeon Kim

PhD Student

T201-2211 Wesbrook Mall

Vancouver, B.C. Canada V6T 2B5

$\mathrm{Tel}:$ : (778) 848-7947

8. You are also permitting Mi-Yeon Kim to access your medical chart in order for her to obtain the relevant information about your congenital heart disease, for example, your diagnosis, heart catheterization results, etc.

9. If you wish to participate in the study and are unable to physically be present at the clinic, you can request that the set of questionnaires be delivered to your home.

\section{Risks and Benefits}

Risks: Although there are no anticipated risks or injury to you as a result of participating in this study, you may find some of the questions upsetting. We will provide you with a list of resources that you can contact should you require support. Your decision to participate or decline in the study will not influence the type, or the quality of care provided at the Adult Congenital Heart Disease Program Outpatient Clinic. 
Benefit: Although you will not directly benefit from participating in this study, it is our hope that the findings of this study will be used in the future to help those who are experiencing or at risk for developing emotional distress as a result of living with congenital heart disease.

\section{Confidentiality}

Your confidentiality will be respected. Information that discloses your identity will be not released without your consent unless required by law or regulation. However, research records and medical records identifying you may be inspected in the presence of the investigator or his or her designate, by Health Canada, and the University of British Columbia-Providence Health Care (UBC-PHC) Research Ethics Board for the purposes of monitoring the research. No records that identify you by name or initials will be allowed to leave the investigator's office.

\section{Rights and Compensation}

By signing this form, you do not give up any of your legal rights and you do not release the study investigator(s) or other participating institutions from their legal and professional duties. There will be no cost to you for participation in this study.

\section{Contact Information to Inquire About Your Rights as a Research Participant}

If you have any concerns about your rights as a research subject and/or your experiences while participating in this study, contact the Research Subject Information Line in the University of British Columbia Office of Research Services at (604) 822-8598 or the Chair of the UBC-PHC Research Ethics Board at (604) 682-2344 ext 63496.

\section{Contact Information}

If you have any questions about this study, you are welcome to ask Mi-Yeon at any time or contact her at (778) 848-7947, or her research supervisor Dr. Joy Johnson at (604) 822-7435.

If you wish, a copy of the results of this study will be provided to you after the completion of the study.

\section{Voluntary Consent}

Your participation in this study is entirely voluntary and you are free to withdraw from the study at any time with no consequences. Your decision to not participate in the study will not jeopardize your relationship with any members of the health care team of the Adult Congenital Heart Disease Program or any treatment(s) you receive. 


\section{Relationship between types of social support, coping styles, and psychological distress in individuals living with congenital heart disease}

Consent

- I have had the opportunity to ask questions and understand the information given above.

- I understand that all of the information collected will be kept confidential.

- I understand that my participation in this study is voluntary and that I am completely free to refuse to participate or to withdraw from this study at any time without changing in any way the quality of care that I receive.

- I understand that I am not giving up any of my legal rights as a result of signing this consent form.

- I understand that while I am encouraged to answer all questions, I am not obliged to answer any questions that I feel uncomfortable answering.

- I have read this form and I freely consent to participate in this study. I understand that I will be given a copy of this signed and dated consent form.

Printed name of subject

Signature

Date

Printed name of

Principal or Co-Investigator

Signature

Date 


\title{
Appendix B
}

\author{
Questionnaires
}


UBC THE UNIVERSITY $\mathrm{OF}$

B R I T I S H C O L U M B I A

\section{CLIENT QUESTIONNAIRES}

Participant Number:

Date:

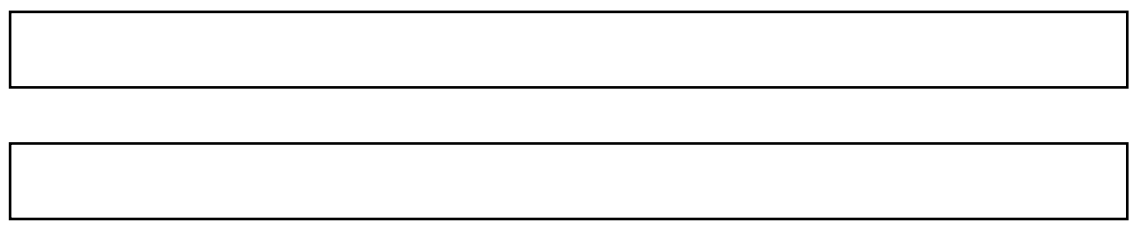

The data collected in these questionnaires is confidential.

When completed, please insert the questionnaires into the envelope provided and leave it with the receptionist at the Adult Congenital Heart Disease Program Clinic. Thank you very much for your participation. 


\section{PART I \\ Please check $\sqrt{ }$ beside your answer.}

1. What is your sex? male female

2. What is your age?

3. What is the highest level of schooling you have completed? (please check) some junior high school some college , trade/technical/vocational training high school graduate some post graduate work post graduate degree

4. What is your marital status?

married single divorced common-law

5. How many children do you have? No children number of children living in your home

6. What is your annual income?

\begin{tabular}{|c|c|c|c|}
\hline $0-\$ 10,000$ & $\$ 10,000-20,000$ & $\$ 20,000-30,000$ & $\$ 30,000-40,000$ \\
\hline$\$ 40,000-50,000$ & $\$ 50,000-60,000$ & $\$ 60,000-70,000$ & $>\$ 70,000$ \\
\hline
\end{tabular}

7. What is your employment status?

homemaker full time part-time , college graduate full-time student retired part-time student casual/contract work disability never worked

8. What is your occupation (job)?

9. What is your religious preference? an orthodox church (for example, Greek or Russian Orthodox)

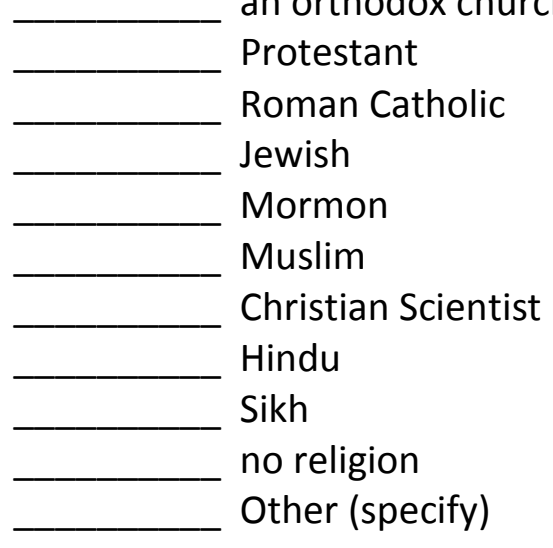


10. Have you ever been diagnosed with anxiety? Yes

No

depression? Yes No

stress-related problem or another mental health problem? Yes

If yes to any of the above, do you still experience the condition(s)? Yes

No

If

No

11. In general, would you say your health is:

excellent very good

good

fair

poor

12. Compared to one year ago, how would you rate your health in general now?

much better now somewhat better about the same somewhat worse much worse than 1 year ago now than 1 year ago as 1 year ago than 1 year ago than 1 year ago 


\section{PART II}

\section{BECK ANXIETY INVENTORY*}

Below is a list of commonly experienced symptoms. Please carefully read each item in the list. Indicate how much you have been bothered by that symptom during the past month, including today, by circling the number in the corresponding space in the column next to each symptom.

\begin{tabular}{|c|c|c|c|c|c|}
\hline & & $\begin{array}{c}\text { Not at } \\
\text { all }\end{array}$ & $\begin{array}{l}\text { Mildly but it } \\
\text { didn 't bother } \\
\text { me much }\end{array}$ & $\begin{array}{l}\text { Moderately - } \\
\text { it wasn 't } \\
\text { pleasant at } \\
\text { times }\end{array}$ & $\begin{array}{l}\text { Severely - it } \\
\text { bothered me } \\
\text { a lot }\end{array}$ \\
\hline & example & 0 & 1 & 2 & 3 \\
\hline 1. & Numbness or tingling & 0 & 1 & 2 & 3 \\
\hline 2. & Feeling hot & 0 & 1 & 2 & 3 \\
\hline 3. & Wobbliness in legs & 0 & 1 & 2 & 3 \\
\hline 4. & Unable to relax & 0 & 1 & 2 & 3 \\
\hline 5. & Fear of worst happening & 0 & 1 & 2 & 3 \\
\hline 6. & Dizzy or lightheaded & 0 & 1 & 2 & 3 \\
\hline 7. & Heart pounding/racing & 0 & 1 & 2 & 3 \\
\hline 8. & Unsteady & 0 & 1 & 2 & 3 \\
\hline 9. & Terrified or afraid & 0 & 1 & 2 & 3 \\
\hline 10. & Nervous & 0 & 1 & 2 & 3 \\
\hline 11. & Feeling of choking & 0 & 1 & 2 & 3 \\
\hline 12. & Hands trembling & 0 & 1 & 2 & 3 \\
\hline 13. & Shaky/unsteady & 0 & 1 & 2 & 3 \\
\hline 14. & Fear of losing control & 0 & 1 & 2 & 3 \\
\hline 15. & Difficulty in breathing & 0 & 1 & 2 & 3 \\
\hline 16. & Fear of dying & 0 & 1 & 2 & 3 \\
\hline
\end{tabular}

* The name of each instrument was not identified in the questionnaires. 


\begin{tabular}{|l|l|c|c|c|c|}
\hline & Not at all & $\begin{array}{l}\text { Mildly but it } \\
\text { didn`t bother } \\
\text { me much }\end{array}$ & $\begin{array}{l}\text { Moderately - it } \\
\text { wasn`t } \\
\text { pleasant at } \\
\text { times }\end{array}$ & $\begin{array}{l}\text { Severely - it } \\
\text { bothered me } \\
\text { a lot }\end{array}$ \\
\hline 17. & Scared & 0 & 1 & 2 & 3 \\
\hline 18. & Indigestion & 0 & 1 & 2 & 3 \\
\hline 19. & Faint/lightheaded & 0 & 1 & 2 & 3 \\
\hline 20. & Face flushed & 0 & 1 & 2 & 3 \\
\hline 21. & Hot/cold sweats & 0 & 1 & 2 & 3 \\
\hline
\end{tabular}




\section{BECK DEPRESSION INVENTORY}

Please read each question carefully and choose the one statement in each group that best describes the way you have been feeling during the past month including today. Circle the number beside the statement you have picked. If several statements in the group seem to apply equally well, circle the highest number for that group. Do not choose more than one statement for any group, including item \#16 (changes in sleeping pattern) or item \#18 (changes in appetite).

1. Sadness

0 . I do not feel sad.

1. I feel sad much of the time.

2. I am sad all the time.

3. I am so sad or unhappy that I can`t stand it.

2. Pessimism

0 . I am not discouraged about my future.

1. I feel more discouraged about my future than I used to be

2. I do not expect things to work out for me.

3. I feel my future is hopeless and will only get worse

3. Past Failure

0 . I do not feel like a failure.

1. I have failed more than I should have.

2. As I look back, I see a lot of failures.

3. I feel I am a total failure as a person.

4. Loss of Pleasure

0 . I get as much pleasure as I ever did from the things I enjoy.

1. I don't enjoy things as much as I used to.

2. I get very little pleasure from the things I used to enjoy.

3. I can't get any pleasure from the things I used to enjoy.

5. Guilty Feelings

0 . I don`t feel particularly guilty.

1. I feel guilty over many things I have done or should have done.

2. I feel quite guilty most of the time.

3. I feel guilty all of the time. 
6. Punishment Feelings

0 . I don't feel I am being punished

1. I feel I may be punished

2. I expect to be punished.

3. I feel I am being punished.

7. Self-Dislike

0 . I feel the same about myself as ever.

1. I have lost confidence in myself.

2. I am disappointed in myself.

3. I dislike myself.

8. Self-Criticalness

0 . I don't criticize or blame myself more than usual.

1. I am more critical of myself than I used to be.

2. I criticize myself for all of my faults.

3. I blame myself for everything bad that happens.

9. Suicidal Thoughts or Wishes

0 . I don't have any thoughts of killing myself.

1. I have thoughts of killing myself, but I would not carry them out.

2. I would like to kill myself.

3. I would kill myself if I had the chance.

10. Crying

0 . I don't cry anymore than I used to.

1. I cry more than I used to.

2. I cry over every little thing.

3. I feel like crying, but I can't.

11. Agitation

0 . I am no more restless or wound up than usual.

1. I feel more restless or wound up than usual.

2. I am so restless or agitated that it's hard to stay still.

3. I am so restless or agitated that I have to keep moving or doing something.

12. Loss of Interest

0 . I have not lost interest in other people or activities.

1. I am less interested in other people or things than before.

2. I have lost most of my interest in other people or things.

3. It's hard to get interested in anything. 
13. Indecisiveness

0 . I make decisions about as well as ever.

1. I find it more difficult to make decisions than usual.

2. I have much greater difficulty in making decisions than I used to.

3. I have trouble making any decisions.

14. Worthlessness

0 . I do not feel I am worthless.

1. I don't consider myself as worthwhile and useful as I used to.

2. I feel more worthless as compared to other people.

3. I feel utterly worthless.

15. Loss of Energy

0 . I have as much energy as ever.

1. I have less energy than I used to have.

2. I don't have enough energy to do very much.

3. I don't have enough energy to do anything.

16. Change in Sleeping Pattern (please choose only ONE answer)

0 . I have not experienced any change in my sleeping pattern.

1a. I sleep somewhat more than usual.

1b. I sleep somewhat less than usual.

2a. I sleep a lot more than usual.

2b. I sleep a lot less than usual.

3a. I sleep most of the day.

3b. I wake up 1-2 hours early and can `t get back to sleep.

17. Irritability

0 . I am no more irritable than usual.

1. I am more irritable than usual.

2. I am much more irritable than usual.

3. I am irritable all the time.

18. Change in Appetite (please choose only ONE answer)

0 . I have not experienced any change in my appetite.

1a. My appetite is somewhat less than usual.

1b. My appetite is somewhat greater than usual.

2a. My appetite is much less than before.

2b. My appetite is much greater than usual.

3a. I have no appetite at all.

3 b. I crave food all the time. 
19. Concentration Difficulty

0 . I can concentrate as well as ever.

1. I can't concentrate as well as usual.

2. It's hard to keep my mind on anything for very long.

3. I find I can't concentrate on anything.

20. Tiredness or Fatigue

0 . I am no more tired or fatigued than usual.

1. I get more tired or fatigued more easily than usual.

2. I am too tired or fatigued to do a lot of the things I used to do.

3. I am too tired or fatigued to do most of the things I used to do.

21. Loss of Interest in Sex

0 . I have not noticed any recent change in my interest in sex.

1. I am less interested in sex than I used to be.

2. I am much less interested in sex now.

3. I have lost interest in sex completely. 


\section{WAYS OF COPING QUESTIONNAIRE}

Take a few moments and think about the MOST stressful situation that you have experienced in the past month. By "stressful" we mean a situation that was difficult or troubling for you, either because you felt distressed about what happened, or because you had to use considerable effort to deal with the situation. It might have been a discussion or confrontation with someone close to you, a problem at work, a medical problem, a separation from someone you care about, etc. Think about the details of this stressful situation, for example, where it happened, who was involved, how you acted, and why it was important to you. Questions below are related to this specific stressful situation.

\begin{tabular}{|c|c|c|c|c|c|}
\hline & & $\begin{array}{l}\text { Not } \\
\text { used }\end{array}$ & $\begin{array}{l}\text { Used } \\
\text { some } \\
\text { what }\end{array}$ & $\begin{array}{c}\text { Used } \\
\text { quite a } \\
\text { bit }\end{array}$ & $\begin{array}{c}\text { Used a } \\
\text { great } \\
\text { deal }\end{array}$ \\
\hline 1. & $\begin{array}{l}\text { Just concentrated on what I had to do next - the } \\
\text { next step }\end{array}$ & 1 & 2 & 3 & 4 \\
\hline 2. & $\begin{array}{l}\text { I tried to analyze the problem in order to } \\
\text { understand it better }\end{array}$ & 1 & 2 & 3 & 4 \\
\hline 3. & $\begin{array}{l}\text { Turned to work or substitute activity to take my } \\
\text { mind off things. }\end{array}$ & 1 & 2 & 3 & 4 \\
\hline 4. & $\begin{array}{l}\text { I felt that time would make a difference - the only } \\
\text { thing to do was to wait. }\end{array}$ & 1 & 2 & 3 & 4 \\
\hline 5. & $\begin{array}{l}\text { Bargained or compromised to get something } \\
\text { positive from the situation. }\end{array}$ & 1 & 2 & 3 & 4 \\
\hline 6. & $\begin{array}{l}\text { I did something which I didn't think would work, } \\
\text { but at least I was doing something. }\end{array}$ & 1 & 2 & 3 & 4 \\
\hline 7. & $\begin{array}{l}\text { Tried to get the person responsible to change his or } \\
\text { her mind. }\end{array}$ & 1 & 2 & 3 & 4 \\
\hline 8. & $\begin{array}{l}\text { Talked to someone to find out more about the } \\
\text { situation. }\end{array}$ & 1 & 2 & 3 & 4 \\
\hline 9. & Criticized or lectured myself. & 1 & 2 & 3 & 4 \\
\hline & $\begin{array}{l}\text { Tried not to burn my bridges, but leave things open } \\
\text { somewhat. }\end{array}$ & 1 & 2 & 3 & 4 \\
\hline
\end{tabular}




\begin{tabular}{|c|c|c|c|c|}
\hline & $\begin{array}{l}\text { Not } \\
\text { used }\end{array}$ & $\begin{array}{l}\text { Used } \\
\text { some } \\
\text { what }\end{array}$ & $\begin{array}{c}\text { Used } \\
\text { quite a } \\
\text { bit }\end{array}$ & $\begin{array}{c}\text { Used a } \\
\text { great } \\
\text { deal }\end{array}$ \\
\hline 11. Hoped a miracle would happen. & 1 & 2 & 3 & 4 \\
\hline $\begin{array}{l}\text { 12. Went along with fate; sometimes I just have bad } \\
\text { luck. }\end{array}$ & 1 & 2 & 3 & 4 \\
\hline 13. Went on as if nothing had happened. & 1 & 2 & 3 & 4 \\
\hline 14. I tried to keep my feelings to myself. & 1 & 2 & 3 & 4 \\
\hline $\begin{array}{l}\text { 15. Looked for silver lining, so to speak; tried to look } \\
\text { on the bright side of things. }\end{array}$ & 1 & 2 & 3 & 4 \\
\hline 16. Slept more than usual. & 1 & 2 & 3 & 4 \\
\hline $\begin{array}{l}\text { 17. I expressed anger to the person(s) who caused } \\
\text { the problem. }\end{array}$ & 1 & 2 & 3 & 4 \\
\hline $\begin{array}{l}\text { 18. Accepted sympathy and understanding from } \\
\text { someone. }\end{array}$ & 1 & 2 & 3 & 4 \\
\hline 19. I told myself things that helped me to feel better. & 1 & 2 & 3 & 4 \\
\hline 20. I was inspired to do something creative. & 1 & 2 & 3 & 4 \\
\hline 21. Tried to forget the whole thing. & 1 & 2 & 3 & 4 \\
\hline 22. I got professional help. & 1 & 2 & 3 & 4 \\
\hline 23. Changed or grew as a person in a good way. & 1 & 2 & 3 & 4 \\
\hline $\begin{array}{l}\text { 24. I waited to see what would happen before doing } \\
\text { anything. }\end{array}$ & 1 & 2 & 3 & 4 \\
\hline 25. I apologized or did something to make up. & 1 & 2 & 3 & 4 \\
\hline 26. I made a plan of action and followed it. & 1 & 2 & 3 & 4 \\
\hline 27. I accepted the next best thing to what I wanted. & 1 & 2 & 3 & 4 \\
\hline 28. I let my feelings out somehow. & 1 & 2 & 3 & 4 \\
\hline
\end{tabular}




\begin{tabular}{|c|c|c|c|c|}
\hline & $\begin{array}{l}\text { Not } \\
\text { used }\end{array}$ & $\begin{array}{l}\text { Used } \\
\text { some } \\
\text { what }\end{array}$ & $\begin{array}{c}\text { Used } \\
\text { quite a } \\
\text { bit }\end{array}$ & $\begin{array}{c}\text { Used a } \\
\text { great } \\
\text { deal }\end{array}$ \\
\hline 29. Realized I brought the problem on myself. & 1 & 2 & 3 & 4 \\
\hline $\begin{array}{l}\text { 30. I came out of the experience better than when I } \\
\text { went in. }\end{array}$ & 1 & 2 & 3 & 4 \\
\hline $\begin{array}{l}\text { 31. Talked to someone who could do something } \\
\text { concrete about the problem. }\end{array}$ & 1 & 2 & 3 & 4 \\
\hline $\begin{array}{l}\text { 32. Got away from it for a while; tried to rest or take } \\
\text { a vacation. }\end{array}$ & 1 & 2 & 3 & 4 \\
\hline $\begin{array}{l}\text { 33. Tried to make myself feel better by eating, } \\
\text { drinking, smoking, using drugs or medication, etc. }\end{array}$ & 1 & 2 & 3 & 4 \\
\hline 34. Took a big chance or did something very risky. & 1 & 2 & 3 & 4 \\
\hline $\begin{array}{l}\text { 35. I tried not to act too hastily or follow my first } \\
\text { hunch. }\end{array}$ & 1 & 2 & 3 & 4 \\
\hline 36. Found new faith. & 1 & 2 & 3 & 4 \\
\hline 37. Maintained my pride and kept a stiff upper lip. & 1 & 2 & 3 & 4 \\
\hline 38. Rediscovered what is important in life. & 1 & 2 & 3 & 4 \\
\hline $\begin{array}{l}\text { 39. Changed something so things would turn out all } \\
\text { right. }\end{array}$ & 1 & 2 & 3 & 4 \\
\hline 40. Avoided being with people in general. & 1 & 2 & 3 & 4 \\
\hline $\begin{array}{l}\text { 41. Didn 't let it get to me; refused to think too much } \\
\text { about it. }\end{array}$ & 1 & 2 & 3 & 4 \\
\hline 42. I asked a relative or friend I respected for advice. & 1 & 2 & 3 & 4 \\
\hline 43. Kept others from knowing how bad things were. & 1 & 2 & 3 & 4 \\
\hline $\begin{array}{l}\text { 44. Made light of the situation; refused to get too } \\
\text { serious about it. }\end{array}$ & 1 & 2 & 3 & 4 \\
\hline
\end{tabular}




\begin{tabular}{|c|c|c|c|c|}
\hline & $\begin{array}{l}\text { Not } \\
\text { used }\end{array}$ & $\begin{array}{l}\text { Used } \\
\text { some } \\
\text { what }\end{array}$ & $\begin{array}{c}\text { Used } \\
\text { quite a } \\
\text { bit }\end{array}$ & $\begin{array}{c}\text { Used a } \\
\text { great } \\
\text { deal }\end{array}$ \\
\hline 45. Talked to someone about how I was feeling. & 1 & 2 & 3 & 4 \\
\hline 46. Stood my ground and fought for what I wanted. & 1 & 2 & 3 & 4 \\
\hline 47. Took it out on other people. & 1 & 2 & 3 & 4 \\
\hline $\begin{array}{l}\text { 48. Drew on my past experiences; I was in a similar } \\
\text { situation before. }\end{array}$ & 1 & 2 & 3 & 4 \\
\hline $\begin{array}{l}\text { 49. I knew what had to be done, so I doubled my } \\
\text { efforts to make things work. }\end{array}$ & 1 & 2 & 3 & 4 \\
\hline 50. Refused to believe that it had happened. & 1 & 2 & 3 & 4 \\
\hline $\begin{array}{l}\text { 51. I made a promised to myself that things would be } \\
\text { different next time. }\end{array}$ & 1 & 2 & 3 & 4 \\
\hline $\begin{array}{l}\text { 52. Came up with a couple of different solutions to the } \\
\text { problem. }\end{array}$ & 1 & 2 & 3 & 4 \\
\hline 53. Accepted it, since nothing could be done. & 1 & 2 & 3 & 4 \\
\hline $\begin{array}{l}\text { 54. I tried to keep my feelings from interfering with } \\
\text { other things too much. }\end{array}$ & 1 & 2 & 3 & 4 \\
\hline $\begin{array}{l}\text { 55. Wished that I could change what had happened or } \\
\text { how I felt. }\end{array}$ & 1 & 2 & 3 & 4 \\
\hline 56. I changed something about myself. & 1 & 2 & 3 & 4 \\
\hline $\begin{array}{l}\text { 57. I daydreamed or imagined a better time or place } \\
\text { than the one I was in. }\end{array}$ & 1 & 2 & 3 & 4 \\
\hline $\begin{array}{l}\text { 58. Wished that the situation would go away or } \\
\text { somehow be over with. }\end{array}$ & 1 & 2 & 3 & 4 \\
\hline $\begin{array}{l}\text { 59. Had fantasies or wishes about how things might } \\
\text { turn out. }\end{array}$ & 1 & 2 & 3 & 4 \\
\hline 60. I prayed. & 1 & 2 & 3 & 4 \\
\hline 61. I prepared myself for the worst. & 1 & 2 & 3 & 4 \\
\hline
\end{tabular}




\begin{tabular}{|l|c|c|c|c|}
\hline & $\begin{array}{c}\text { Not } \\
\text { used }\end{array}$ & $\begin{array}{c}\text { Used } \\
\text { some } \\
\text { what }\end{array}$ & $\begin{array}{c}\text { Used } \\
\text { quite a } \\
\text { bit }\end{array}$ & $\begin{array}{c}\text { Used a } \\
\text { great } \\
\text { deal }\end{array}$ \\
\hline 62. I went over in my mind what I would say or do. & 1 & 2 & 3 & 4 \\
\hline $\begin{array}{l}\text { 63. I thought about how a person I admire would } \\
\text { handle this situation and used that as a model. }\end{array}$ & 1 & 2 & 3 & 4 \\
\hline $\begin{array}{l}\text { 64. I tried to see things from the other person's point } \\
\text { of view. }\end{array}$ & 1 & 2 & 3 & 4 \\
\hline 65. I reminded myself how much worse things could \\
be.
\end{tabular}




\section{BERLIN SOCIAL SUPPORT SCALES}

\section{Perceived social support}

\section{Please read the following statements carefully and indicate whether or not you}

agree with each statement.

$\begin{array}{ll}\text { Strongly } & \text { Somewhat } \\ \text { disagree } & \text { disagree }\end{array}$

1. There are some people who truly like me.

2. Whenever I am not feeling well, other people show me that they are fond of me.

3. Whenever I am sad, there are people who cheer me up.

4. There is always someone there for me when I need comforting.
1

1

1

2

1

2

3

4

$\begin{array}{cccc}\text { Strongly } & \text { Somewhat } & \text { Somewhat } & \text { Strongly } \\ \text { disagree } & \text { disagree } & \text { agree } & \text { agree }\end{array}$

5. I know some people upon whom I can always rely.

6. When I am worried, there is someone who helps me.

7. There are people who offer me help when I need it.

8. When everything becomes too much for me to handle, others are there to help me. 


\section{Received social support}

Think about the person who is closest to you, such as your spouse, partner, child, friend, and so on. How did this person react to you during the last month?

$\begin{array}{lccc}\text { Strongly } & \text { Somewhat } & \text { Somewhat } & \text { Strongly } \\ \text { disagree } & \text { disagree } & \text { agree } & \text { agree }\end{array}$

1. This person showed me that he/she loves and accepts me.

2. This person was there when I needed him/her.

3. This person comforted me when I was feeling bad.

4. This person left me alone.

5. This person did not show much empathy

7. This person took care of many things for me.

8. This person made me feel valued and important.

9. This person expressed concern about my condition.

10. This person assured me that I can rely completely on him/her.

11. This person helped me find something positive in my situation.

12. This person suggested activities that might distract me. 


$\begin{array}{lccc}\text { Strongly } & \text { Somewhat } & \text { Somewhat } & \begin{array}{c}\text { Strongly } \\ \text { disagree }\end{array} \\ \text { disagree } & \text { agree } & \text { agree }\end{array}$

14. This person took care of things I could not manage on my own.

2

3

4

15. In general, I am very satisfied with the way this person behaved.

1

2

3

4 


\section{CARDIAC SYMPTOM LIST}

Please think about the last 30 days, that is, the last month. During the past month, how often have you had any of the following types of discomfort?

\begin{tabular}{|l|c|c|c|c|}
\hline & Never & $\begin{array}{c}\text { Only } \\
\text { occasionally }\end{array}$ & $\begin{array}{c}\text { Fairly } \\
\text { often }\end{array}$ & Very often \\
\hline 1. Getting tired easily & 0 & 1 & 2 & 3 \\
\hline 2. Arm or neck pain & 0 & 1 & 2 & 3 \\
\hline $\begin{array}{l}\text { 3. Heaviness, burning or pressure in } \\
\text { your chest }\end{array}$ & 0 & 1 & 2 & 3 \\
\hline 4. Breathlessness & 0 & 1 & 2 & 3 \\
\hline 5. Heart pounding or racing & 0 & 1 & 2 & 3 \\
\hline 6. Chest pain & 0 & 1 & 2 & 3 \\
\hline
\end{tabular}




\section{SHORT HEALTH SURVEY-36v2}

\section{Physical Functioning Subscale}

The following questions are about activities you might do during a typical day. Does your health now limit you in these activities? If so, how much?

\begin{tabular}{|l|c|c|c|}
\hline \multicolumn{1}{|c|}{ Description of activity } & $\begin{array}{l}\text { Yes, limited } \\
\text { a lot }\end{array}$ & $\begin{array}{c}\text { Yes, limited } \\
\text { a little }\end{array}$ & $\begin{array}{c}\text { No, not } \\
\text { limited } \\
\text { at all }\end{array}$ \\
\hline a. $\begin{array}{l}\text { Vigorous activities, such as running, lifting } \\
\text { heavy objects, participating in strenuous } \\
\text { sports }\end{array}$ & 1 & 2 & 3 \\
\hline b. $\begin{array}{l}\text { Moderate activities, such as moving a table, } \\
\text { pushing a vacuum cleaner, bowling, or } \\
\text { playing golf }\end{array}$ & 1 & 2 & 3 \\
\hline c. Lifting or carrying groceries & 1 & 2 & 3 \\
\hline d. Climbing several flights of stairs & 1 & 2 & 3 \\
\hline e. Climbing one flight of stairs & 1 & 2 & 3 \\
\hline f. Bending, kneeling, or stooping & 1 & 2 & 3 \\
\hline g. Walking more than a mile & 1 & 2 & 3 \\
\hline h. Walking several hundred yards & 1 & 2 & 3 \\
\hline i. Walking one hundred yards & 1 & 2 & 3 \\
\hline j. Bathing or dressing yourself & 1 & 2 & 3 \\
\hline
\end{tabular}

Is there any other information that you would like to share with us about your experiences of living with congenital heart disease?

Thank you very much for your participation! 